\title{
Atlas of Experimentally-Induced Neoplasia in Beagle Dogs
}

G.E. Dagle

C.R. Watson

October 1996
RECEIVED

JUN 301997

Q ETI

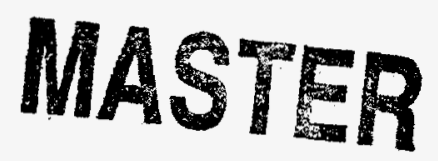

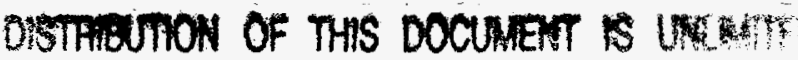

ptl

Prepared for the U.S. Department of Energy

under Contract DE-AC06-76RLO 1830

Pacific Northwest National Laboratory

Richland, Washington 99352 


\title{
DISCLAIMER
}

This report was prepared as an account of work sponsored by an agency of the United States Government. Neither the United States Government nor any agency thereof, nor Battelle Memorial Institute, nor any of their employees, makes any warranty, express or implied, or assumes any legal liability or responsibility for the accuracy, completeness, or usefulness of any information, apparatus, product, or process disclosed, or represents that its use would not infringe privately owned rights. Reference herein to any specific commercial product, process, or service by trade name, trademark, manufacturer, or otherwise does not necessarily constitute or imply its endorsement, recommendation, or favoring by the United States Government or any agency thereof, or Battelle Memorial Institute. The views and opinions of authors expressed herein do not necessarily state or reflect those of the United States Government or any agency thereof.

\author{
PACIFIC NORTHWEST NATIONAL LABORATORY \\ operated by \\ BATTELLE \\ for the \\ UNITED STATES DEPARTMENT OF ENERGY \\ under Contract DE-AC06-76RLO 1830
}




\section{Atlas}

of

\section{Experimentally-Induced}

Neoplasia

-

in

\section{Beagle Dogs}

Contributing Authors

Stephen A. Benjamin

Gerald E. Dagle

Thomas E. Fritz

Nancy A. Gillett

Patrick J. Haley

Fletcher F. Hahn

Bruce A. Muggenburg

Roy R. Pool

Thomas M. Seed

Glenn N. Taylor

David V. Tolle

\section{Editors}

Gerald E. Dagle

Charles R. Watson 



\section{DISCLAMMIER}

Portions of this document may be illegible in electronic image products. Images are produced from the best available original document. 



\title{
Atlas of Experimentally-Induced Neoplasia in the Beagle Dog
}

\author{
Contributing Authors \\ Stephen A. Benjamin, D.V.M., Ph.D. \\ Colorado State University \\ Gerald E. Dagle, D.V.M., Ph.D. \\ Pacific Northwest National Laboratory \\ Currently at Washington State University-Tri Cities \\ Thomas E. Fritz, D.V.M. \\ Argonne National Laboratory \\ Nancy A. Gillett, D.V.M, Ph.D. \\ Inhalation Toxicology Research Institute \\ Currently at Sierra Biomedical Inc. \\ Patrick J. Haley, D.V. M., Ph.D. \\ Inhalation Toxicology Research Institute \\ Currently at Nycomed Inc. \\ Fletcher F. Hahn, D.V.M., Ph.D. \\ Inhalation Toxicology Research Institute \\ Bruce A. Muggenburg, D.V.M., Ph.D. \\ Inhalation Toxicology Research Institute \\ Roy R. Pool, D.V.M., Ph.D. \\ University of California, Davis \\ Thomas M. Seed, Ph.D. \\ Argonne National Laboratory \\ Currently at Armed Forces Radiobiology Research Institute \\ Glenn N. Taylor, D.V.M., Ph.D. \\ University of Utah \\ David V. Tolle, M.S. \\ Argonne National Laboratory \\ Edited by \\ Gerald E. Dagle, D.V.M., Ph.D. \\ Charles R. Watson, Ph.D. \\ Pacific Northwest National Laboratory
}





\section{PREFACE}

\section{PURPOSE AND GENESIS OF THE ATLAS}

Beagle dogs have been utilized extensively in biomedical research (Andersen 1970, Stannard 1988, Thompson 1989). The U.S. Department of Energy's (DOE) Office of Health and Environmental Research (OHER) has sponsored life-span dose-effect radiation studies in beagles at Argonne National Laboratory (ANL); at the Laboratory for Energy-Related Health Research (LEHR), University of California, Davis; at Lovelace Inhalation Toxicology Research Institute (ITRI); at Pacific Northwest National Laboratory (PNNL); and at the Radiobiology Laboratory, University of Utah. Similar studies were conducted at the Collaborative Radiological Health Laboratory, Colorado State University. Reasons for choosing the beagle over other breeds of dogs included their convenient size, good disposition, and low natural incidence of bone tumors. All the beagles used for these studies have been of purebred stock and were raised in the individual laboratories.

Because results from studies in the various laboratories were to be compared, all the investigators strove to use similar nomenclature and criteria to describe biological effects. For this reason, pathologists from these laboratories met on five occasions between 1976 and 1977 to discuss nomenclature and histologic criteria for diagnoses. At these meetings, criteria were discussed for histopathologic description of lesions in bone, liver, lung, hematopoietic and lymphoid tissues, mammary gland, pituitary, testis, and thyroid. To provide further assurance of cooperation among the DOE laboratories involved, DOE organized several Task Groups in 1985, composed of staff members from the laboratories. The Task Group on Biological Effects was asked to standardize nomenclature and diagnostic criteria for pathology; this beagle pathology atlas is the result of that request. The nomenclature used by pathologists in the various laboratories generally follows that given by the World Health Organization (WHO 1974; 1976); however, modifications are occasionally made to accommodate situations specific to beagles. The purpose of this pathology atlas is to document and explain the diagnostic criteria used in these studies.

The atlas describes target organs of particular interest: lungs for radionuclides delivered by inhalation; bones for bone-seeking radionuclides (delivered by inhalation, injection, or oral administration); and hematopoietic and other soft tissues for external irradiation. It should be noted that, in life-span studies, individual lesions generally cannot be considered radiation-induced; the association between radiation and lesions in radiation-exposed beagles is generally made using statistical methods.

This atlas emphasizes histopathologic observations using the light microscope and routine tissue preparation procedures and stains. The text for each organ system chapter was written by collaborating pathologists and was then reviewed by the other authors.

A large database has accumulated on beagles that were studied in these laboratories. For purposes of computer storage and retrieval, a Systematized Nomenclature of Medicine (SNOMED) diagnostic code (a special adaptation of the Systematized Nomenclature of Veterinary Medicine [SNOVET] codes by Palotay and Rothwell 1984) has been provided for each morphologic diagnosis.

The objective of these efforts is to ensure confidence in the comparisons of research results of dose-effect studies in beagle dogs performed in various laboratories. The results of these studies will continue to be published as peer-reviewed joumal articles, and many details of the histopathologic diagnoses will be published in those reports. It is also anticipated that newer methods will become available to better understand morphologic changes influencing diagnoses. 


\section{ROLE OF THE NATIONAL RADIOBIOLOGY ARCHIVES}

Chapters for the atlas were written over a period of several years. Those for leukemia and thyroid were completed in 1989. Four were complete by the end of 1992. As the chapters were received, they were edited and standardized in word-processing files. Proofreading copies of the leukemia, thyroid, bone, and liver chapters were returned to the contributing authors and circulated among the others. It was realized from the start that the atlas would be an expensive publication because of the large number of color and half-tone figures. When the atlas was complete, there were insufficient funds in the lifespan beagle study project at PNNL for publication. Therefore, the manuscript and photomicrographs were transferred to the National Radiobiology Archives (NRA) project at PNNL. [The NRA is now part of the U.S. Transuranium and Uranium Registries (USTUR) at Washington State University-Tri Cities (WSU-TC)].

As the beagle studies were being completed, the DOE instigated an archival project to assure that detailed research records would be available for future analysis. The NRA, operated by PNNL for the $D O E$, is a repository for information about tens of thousands of individual rodents and other animals which were used in long-term radiobiology studies conducted by the U.S. government over the last 50 years. The mission of the NRA is to gather, organize, and catalog data, original documents, and tissues related to DOE radiobiology life-span studies. The NRA has three tasks:

- operate an interlaboratory computerized information system containing a dose-and-effects database to summarize data on individual animals, an inventory database, and a bibliographic database;

- establish a document archive of original (or "record copy") research materials, such as logbooks, clinical notes, radiographic films, and pathologists' observations;

- operate a specimen archive of histopathology blocks, slides, and tissue samples.

The NRA concentrated initially on studies of beagle dogs exposed to ionizing radiation at five DOE laboratories; results for each of more than 6000 life-span-observation dogs have been transferred and are available. Details of major studies comparing strains of mice were transferred from Oak Ridge National Laboratory and Brookhaven National Laboratory; results for nearly 30,000 mice are available. Additionally, the NRA recently acquired records and specimens from a life-span study of almost 4000 rats that inhaled plutonium at PNNL. Life-span biokinetics data on over 300 nonhuman primates are also available.

At its inception, in 1989, it appeared that the primary task of the NRA was to gather electronic information related to radiobiology studies and combine it into a master database system. The studies, conducted over a long time span in many different laboratories, each used a different approach to data management, ranging from handwritten laboratory notebooks to elaborate computerized database management systems. The DOE wanted to be able to combine results from studies in a unified electronic format accessible from a microcomputer. At that time the NRA task was to design and populate a unified database structure.

As experience was gained with users of the combined database, it became very evident that the NRA is much more than a combined, unified database. The NRA is a living, value-added organization which strives to preserve original material and, at the same time, makes it readily available to new users. The users need access to original data files and documentation to supplement their use of the unified database, and we must carefully distinguish between the original information and the value-added standardization provided by the archival service.

The NRA is selective in scope. The goal is to characterize and preserve the key radiobiological experiments, especially those that are large and costly, which will never be repeated. New studies are added at the concurrence of the advisory committee. When a study is nominated for inclusion, we consider the availability of materials. The optimal approach is to be able to collect electronic data, 
written documents, histopathology slides and paraffin blocks, tissues, radiographs, and other materials-in order to provide the entire spectrum of materials for interpretation by new analytical or statistical techniques. In other words, a slide collection is useless without extensive supporting documentation, preferably in the form of a computer database. On the other hand, a database without a slide collection can provide a valuable addition to our collection because it can be combined with other databases in cross-cutting analyses.

The formation phase of the NRA is essentially complete; no significant studies are expected to be added. A detailed description of NRA holdings, and those of parallel archival efforts in Europe and Japan is available (Gerber et al. 1996). The project is phasing into a maintenance/user service mode and its collections and service activities are being merged with those of the USTUR under the direction of Dr. Ronald L. Kathren at WSU.

The NRA was the logical custodian of the atlas because it maintains the centralized copy of the beagle data. Furthermore, as described in the Appendix, the NRA had been involved over the years with most of the authors in efforts to develop the SNODOG beagle-specific variant of SNOVET. It was planned to supply Archives users with photocopies of the atlas text and selected photomicrographs on an individual basis in conjunction with future retrievals of subsets of the database. However, in the summer of 1996, it became apparent that cost savings from other NRA activities were available and we determined to produce the atlas in full as envisioned by its authors.

\section{EDITORIAL CONSIDERATIONS}

Prepublication editing in 1996 included further standardization of format, consistent numbering of figures, addition of a list of acronyms and an index. A table was added to each chapter to present the nomenclature, a list of figures, and corresponding SNODOG codes (used in the NRA database) in a convenient format.

There are five chapters dealing with bone, myeloid leukemia, liver, lung, and thyroid. Each chapter is self-contained and consists of narrative descriptions and references, followed by a group of figures. Although it might have been more convenient for the reader if figures were interposed within the text, it was more economical to place them together at the end of each chapter.

The original intent was to provide the atlas in loose-leaf format with 3-ring binders to facilitate periodic supplements. For a number of reasons, it is highly unlikely that any formal, supplements will be forthcoming; therefore, the present binding was selected. It is the authors' intent to continue to collaborate and advise each other of changes and additions. Readers are urged to consult with chapter authors about possible postpublication changes in nomenclature or diagnostic technique. To facilitate such communication, the Author Index contains current addresses.

The delay between chapter preparation and publication complicated the review process for the completed atlas. Since there was insufficient time for review by the contributing authors, and since many of the contributing authors have relocated, final proofreading was performed by Chuck Watson and Dvara-Lee Felton in August, 1996. 


\section{REFERENCES}

Andersen, A. C., ed. 1970. The Beagle as an Experimental Dog. Iowa State University Press, Ames, IA.

Gerber, G.B, Okada, S., Sugahara, T. and Watson, C.R. 1996. International Radiobiology. Archives of Long-Term Animal Studies I. Descriptions of Participating Institutions and Studies DOE/RL 96-72 Office of Scientific and Technical Information, Springfield, VA.

Palotay, J. L., and Rothwell, D. J., ed. 1984. SNOVET Systematized Nomenclature of Medicine Microglossary for Veterinary Medicine. American Veterinary Medical Association, Schaumburg, IL.

Stannard, J. N. 1988. Radioactivity and Health, A History (DE88013791) Office of Scientific and Technical Information, Springfield, VA.

Thompson, R. C. 1989. Life-Span Effects of Ionizing Radiation in the Beagle Dog. Pacific Northwest National Laboratory, Richland, WA.

World Health Organization. 1974. International histological classification of tumours of domestic animals. Bull. WHO 50:1-144.

World Health Organization. 1976. International histological classification of tumours of domestic animals. Bull. WHO 50:145-282.

\section{ACKNOWLEDGMENTS}

The editors are deeply appreciative of the editorial efforts of Raymond W. Baalman, Jr. and Dvara-Lee Felton. They were responsible for organization and preservation of the text and illustrations for many years. 


\section{CONTENTS}

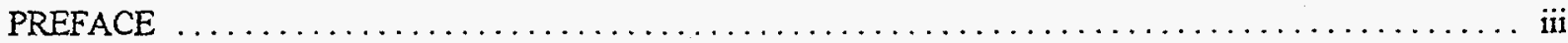

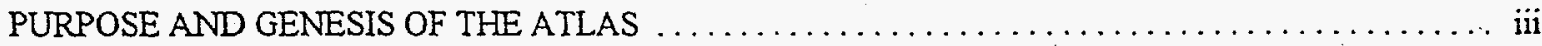

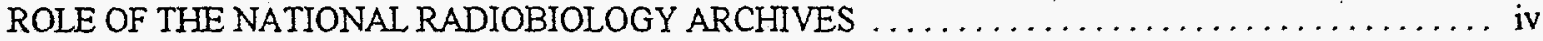

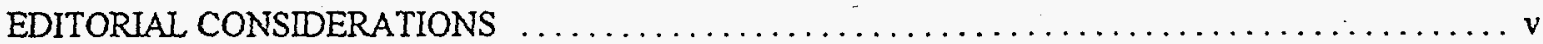

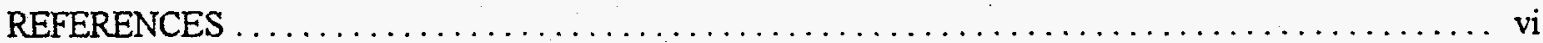

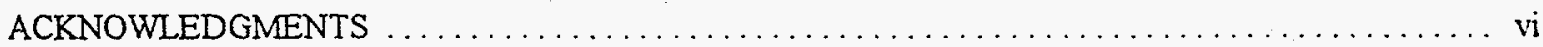

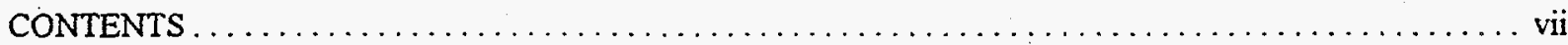

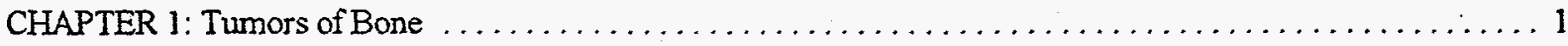

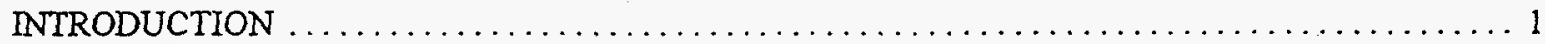

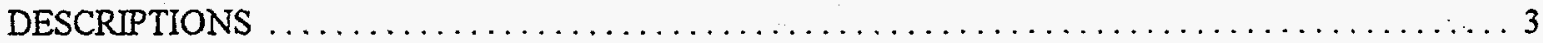

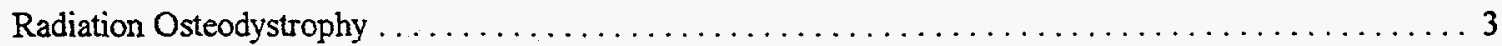

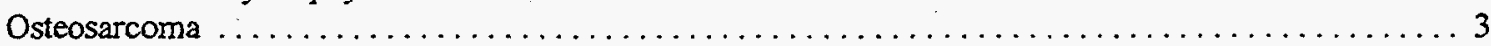

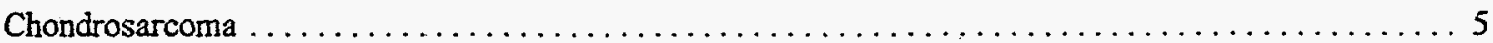

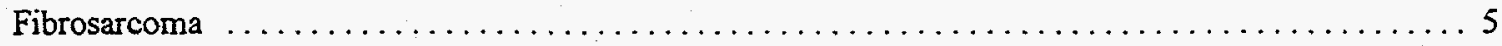

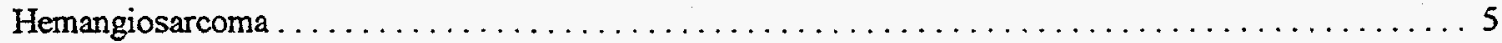

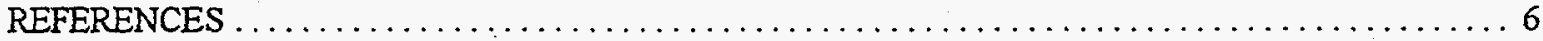

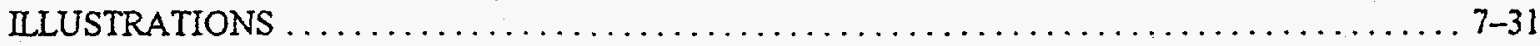

CHAPTER 2: Radiation-Induced Myeloid Leukemia $\ldots \ldots \ldots \ldots \ldots \ldots \ldots \ldots \ldots \ldots \ldots \ldots \ldots \ldots \ldots \ldots \ldots \ldots$

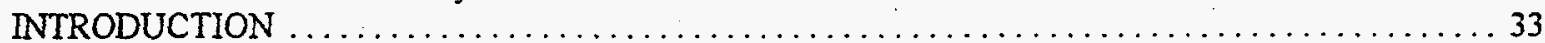

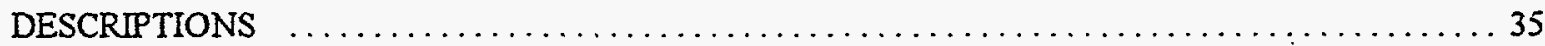

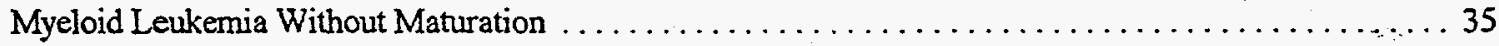

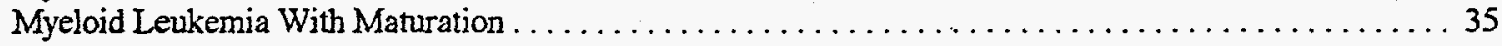

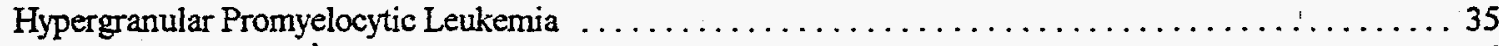

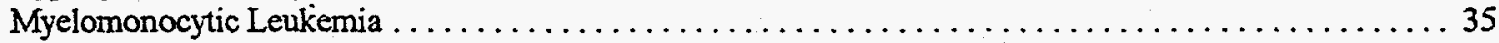

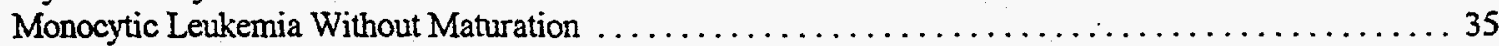

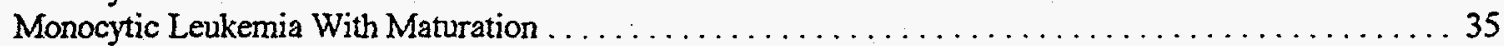

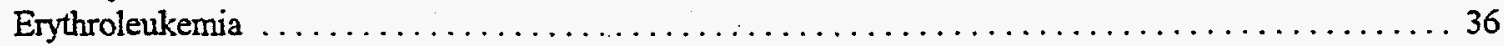

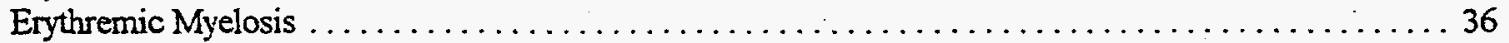

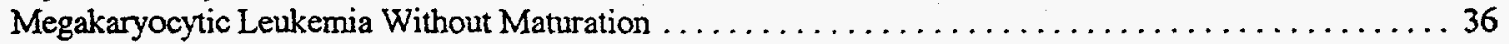

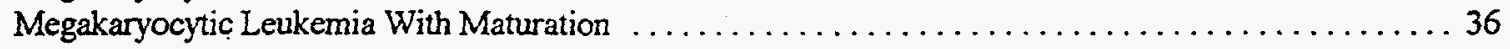

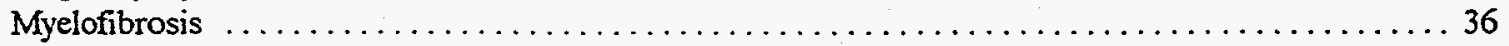

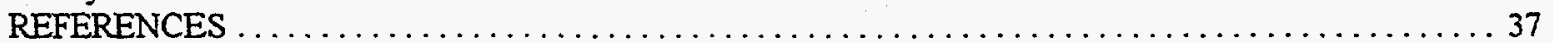

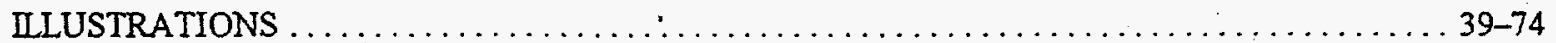

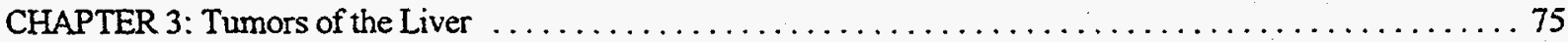

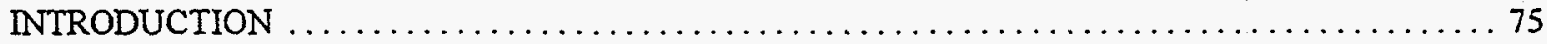

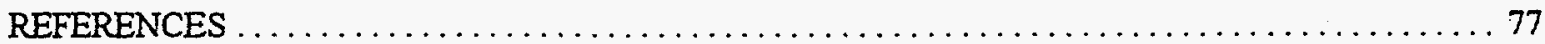

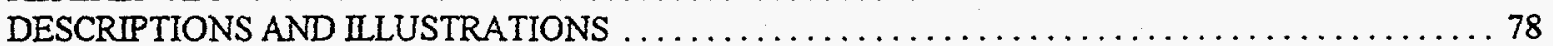

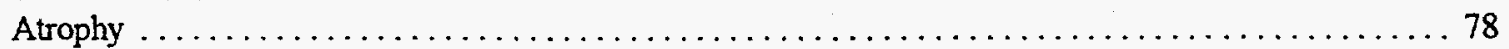

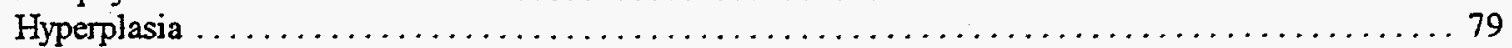

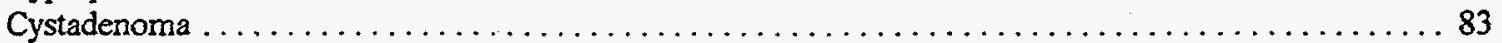

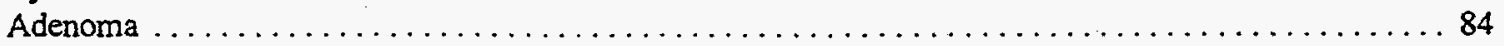

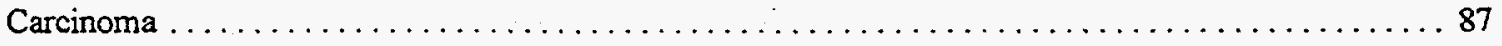

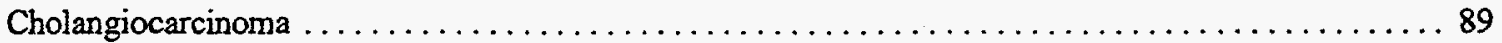

Mesothelioma ...................................................... 93

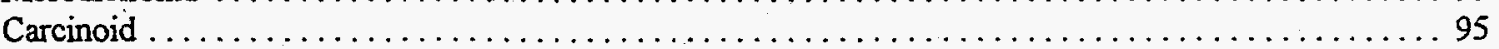

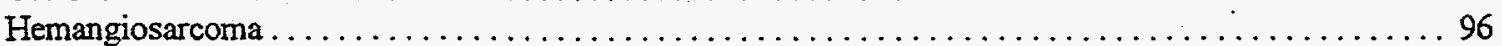

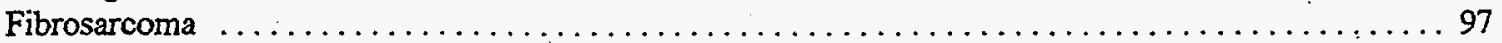

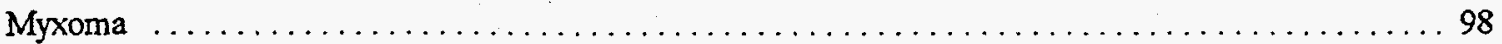

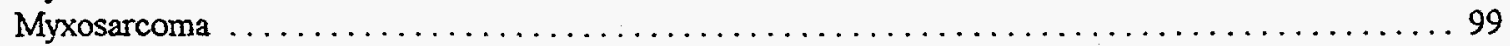

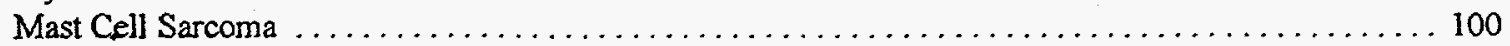




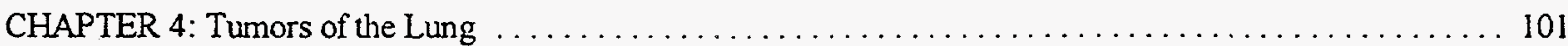

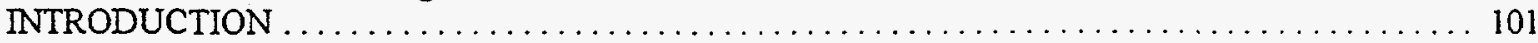

DESCRIPTIONS $\ldots \ldots \ldots \ldots \ldots \ldots \ldots \ldots \ldots \ldots \ldots \ldots \ldots \ldots \ldots \ldots \ldots \ldots \ldots \ldots \ldots \ldots$

Epidermoid Carcinoma (Squamous Cell Carcinoma) $\ldots \ldots \ldots \ldots \ldots \ldots \ldots \ldots \ldots \ldots \ldots \ldots$

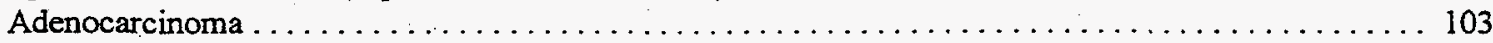

Adenosquamous Carcinoma (Combined Carcinoma) . . . . . . . . . . . . . . . . . 103

Anaplastic Carcinomas . . . . . . . . . . . . . . . . . . . . . . . . . . . . . . . 103

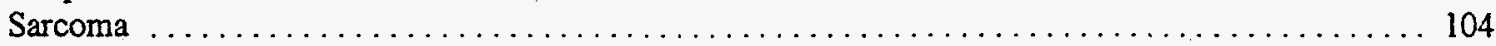

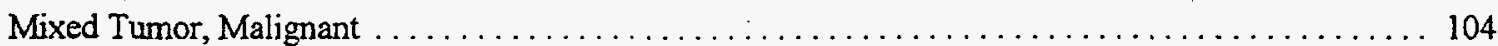

Carcinoid Tumors . . . . . . . . . . . . . . . . . . . . . . . . . . . . . . . 104

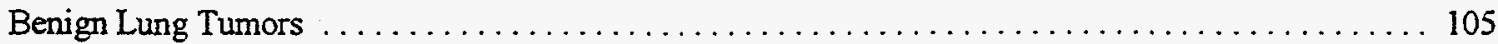

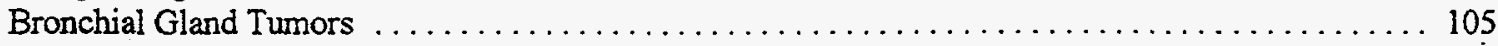

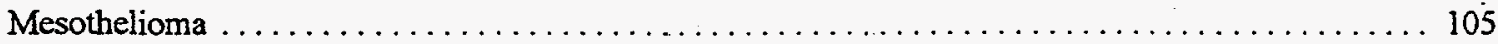

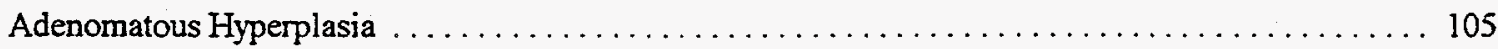

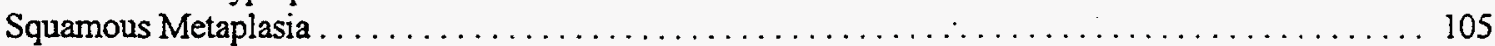

Chronic Radiation Pneumonitis $\ldots \ldots \ldots \ldots \ldots \ldots \ldots \ldots \ldots \ldots \ldots \ldots \ldots \ldots \ldots \ldots \ldots \ldots \ldots \ldots$

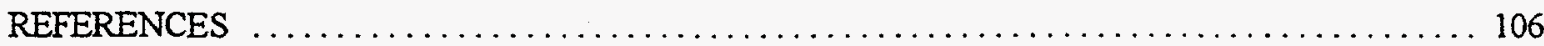

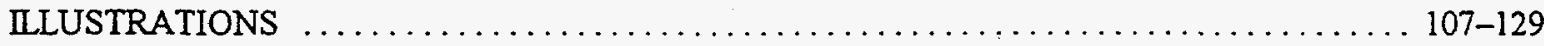

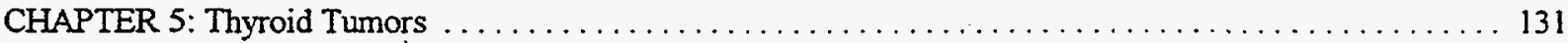

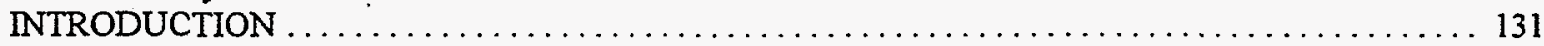

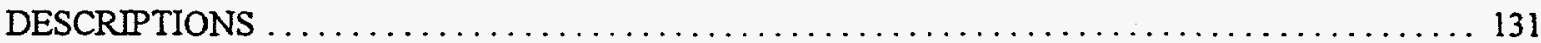

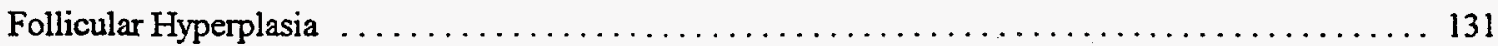

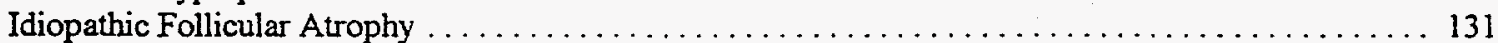

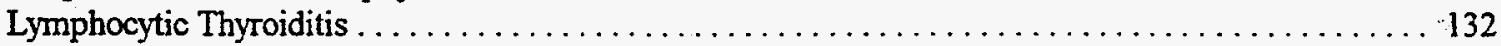

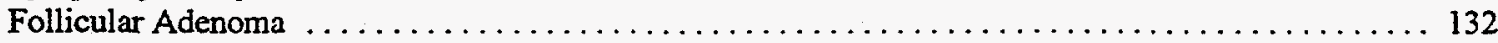

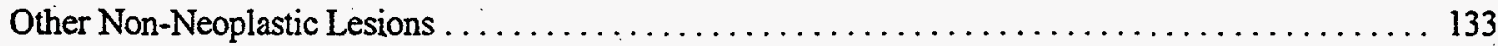

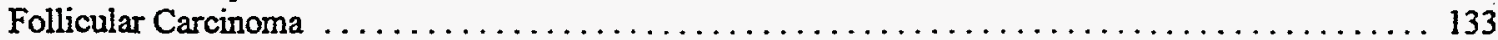

Solid Carcinoma $\ldots \ldots \ldots \ldots \ldots \ldots \ldots \ldots \ldots \ldots \ldots \ldots \ldots \ldots \ldots \ldots \ldots \ldots \ldots \ldots \ldots \ldots \ldots \ldots \ldots$

Papillary Carcinoma . . . . . . . . . . . . . . . . . . . . . . . . . . . . . . . . 134

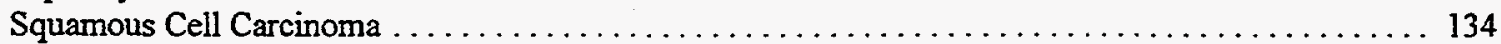

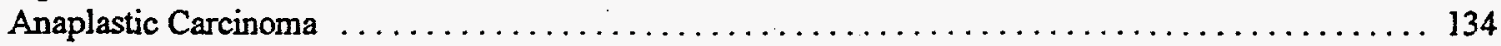

C-Cell Carcinoma . . . . . . . . . . . . . . . . . . . . . . . . . . . . . . . . . . . . . 134

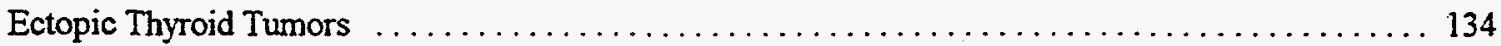

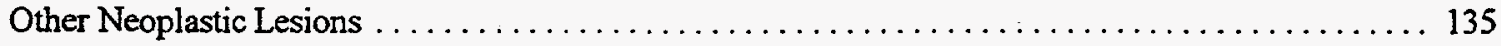

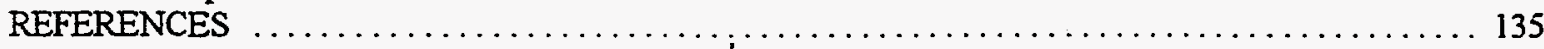

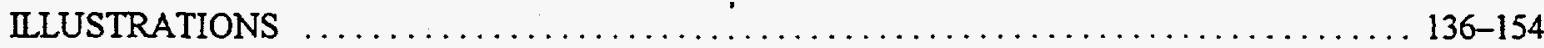

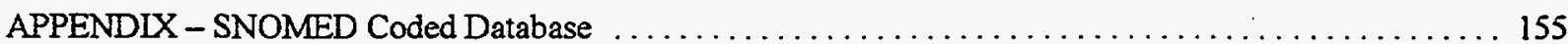

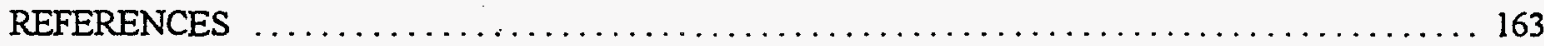

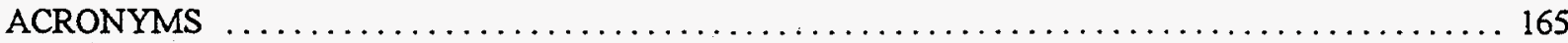

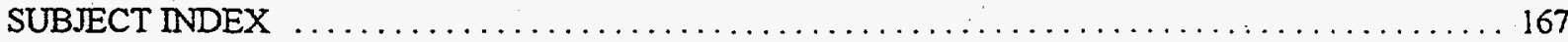

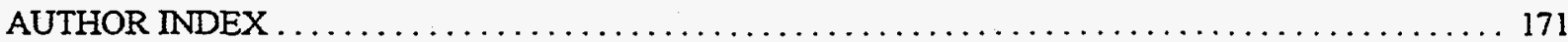




\title{
CHAPTER 1: Tumors of Bone
}

\author{
Nancy A. Gillett \\ Inhalation Toxicology Research Institute \\ (Currently at Sierra Biomedical Inc.) \\ Roy R. Pool \\ University of California, Davis \\ Bruce A. Muggenburg \\ Inhalation Toxicology Research Institute
}

\section{INTRODUCTION}

Many of the radionuclides used in the various life-span studies performed in beagle dogs were bone-seekers, for which the predominant late effect observed was primary bone cancer. This chapter discusses the types of bone tumors observed. Because beagles have been shown to have a very low rate of spontaneous bone cancer, the vast majority of these tumors are presumed to be radiationinduced (Pool, Hilliams, and Goldman 1973). The classification scheme used for the bone tumors is that described by Pool in the $3^{\text {rd }}$ edition of Tumors of Domestic Animals (Pool, 1990). The majority of the spontaneous tumor phenotypes are represented among the radiation-induced tumors and will be discussed subsequently. Those tumor phenotypes that were not observed in the beagle life-span studies are not presented in this chapter. Radiographs are included where possible; radiographs frequently are invaluable in the clinical diagnosis of bone cancer and illustrate the lytic (destructive), productive, or mixed (destructive and productive) bone lesions produced by the various types of bone tumors. Radiographs also delineate the anatomic location of bone tumors, which can be characteristic of the bone tumor phenotype.

The classification used here is that developed by Pool as a modified version of the one developed by Dahlin and Unni (1977) for categorizing primary bone tumors in humans (Table 1.1).

Osteosarcomas, primary malignant bone tumors in which the neoplastic cells produce tumor osteoid and tumor bone (calcified tumor osteoid), display a wide range of histologic and radiographic patterns, depending upon the amount and quality of matrix produced. Although differences in clinical behavior among the various subclasses of osteosarcoma have not been documented in domestic animals, this classification scheme has been useful for distinguishing typical histological patterns in bone neoplasms observed following exposure to bone-seeking radionuclides. Different types of primary bone tumors have developed following exposure of animals to various types of bone-seeking radionuclides given by different routes; these differences are being studied to determine the possible mechanisms of pathogenesis.

Included in this chapter is a brief description of the syndrome of bone lesions termed radiation osteodystrophy. This lesion was frequently observed in bones from radiation-exposed dogs and, in some studies, was consistently associated with sites of tumor production (Pool, Hilliams, and Goldman 1973; Pool, Mortan, and Parks 1983; Nilsson, Morgan, and Book 1985; Hahn et al. 1981). Radiation osteodystrophy has been suggested as a key factor in the induction of the osteosarcomas observed in some of these studies. 
Additional non-neoplastic lesions of bone were not observed routinely; those that did occur were rare spontaneous bone lesions. These lesions have been described in standard veterinary pathology texts and are not discussed in this chapter. Table 1.1 lists the lesions described, corresponding figure numbers, and SNOMED database codes.

Table 1.1. Bone Neoplasia nomenclature, index of figures, and SNODOG database codes.

\begin{tabular}{|c|c|c|}
\hline Nomenclature & Figure(s) & $\begin{array}{c}\text { SNODOG } \\
\text { Morphology } \\
\text { Code }^{\text {e }}\end{array}$ \\
\hline \multicolumn{3}{|l|}{ Non-neoplastic } \\
\hline Radiation Osteodystrophy & $1.1-4$ & M560002 \\
\hline \multicolumn{3}{|l|}{ Neoplastic } \\
\hline Osteosarcoma & & M918030 \\
\hline Poorly Differentiated Osteosarcoma & 1.5 & M918031 \\
\hline Osteoblastic Osteosarcoma & & M918730 \\
\hline Nonproductive Osteoblastic Osteosarcoma & $1.6-7$ & M918731 \\
\hline \multicolumn{3}{|l|}{ Osteosarcoma } \\
\hline Productive Osteoblastic Osteosarcoma & $1.9-10$ & M918733 \\
\hline Chondroblastic Osteosarcoma & $1.11-12$ & M918130 \\
\hline Fibroblastic Osteosarcoma & 1.13 & M918230 \\
\hline Telangiectatic Osteosaroma & $1.14-15$ & M918330 \\
\hline Giant Cell Type Osteosarcoma & 1.16 & M918630 \\
\hline Combined-Type Osteosarcoma & $1.17-18$ & M918530 \\
\hline Chondrosarcoma & $1.19-21$ & M922030 \\
\hline Fibrosarcoma & $1.22-24$ & M881030 \\
\hline Hemangiosarcoma & 1.25 & M912030 \\
\hline Giant Cell Tumor of Bone & na & M925030 \\
\hline Multilobular Tumor of Bone (Chondroma Rodens) & na & \\
\hline Liposarcoma & na & M885030 \\
\hline \multicolumn{3}{|l|}{ Lymphoid and Myelomatous Tumors } \\
\hline Lymphosarcoma & na & M961030 \\
\hline Reticulum Cell Sarcoma (Histocytic Lymphoma) & na & M964030 \\
\hline Plasma Cell Myeloma (Multiple Myeloma) & na & M973030 \\
\hline
\end{tabular}




\section{DESCRIPTIONS}

\section{Radiation Osteadystrophy}

Radiation osteodystrophy is the term used to describe a combination of bone lesions that has been associated with irradiation of bone by internally deposited alpha- or beta-emitting radionuclides. The syndrome is characterized by multiple areas of bone infarction, macroresorption cavities, new bone formation, and marrow fibrosis (Figure 1.1). Bone infarction is identified by empty osteocyte lacunae that may be present within osteons having occluded blood vessels or in osteons having an apparently normal vascular supply. Macroresorptive cavities differ from normal resorption cavities by having larger diameters, more eccentricity of the path of the resorption cavity and orientation to preexisting vascular pathways, and outpockets of osteoclastic resorption extending from the walls of the major resorptive cavity (Figures $1.2 ; 1.3$ ). The cavities are primarily limited to the diaphyseal cortex. New bone formation occurring in response to alterations in normal cortical structure may be evident in a subperiosteal or inner cortical location (Figure 1.4). Peritrabecular new bone formation is also prominent in some cases. A proliferative, fibro-osseous response is frequently seen in the marrow and often fills some of the resorptive cavities. This response resembles those seen with fibrous dysplasia, ossifying fibroma and osteoblastoma. In severe lesions of radiation osteodystrophy, periosteal involvement may be present and is characterized by periosteal new bone formation and fibrosis. The term radiation osteodystrophy is used to describe this entire spectrum of altered bone remodeling.

The pathogenesis of the lesion appears to be a disruption of the normal remodeling of bone. The resorptive phase of remodeling is "uncoupled" from the bone formation phase. Precursors of osteoclasts, the cells responsible for bone resorption, are located in the bone marrow. Because of the distance between these cells and the site of radioisotope deposition in the bone matrix, the osteoclast precursors are unaffected by the radiation, and bone resorption is essentially normal. In contrast, when the precursor cells responsible for bone formation are irradiated, there is subsequent disruption of normal bone formation. In several studies, radiation osteodystrophy was consistently found at sites of bone tumor formation, suggesting a possible etiologic link between the two lesions. In some studies, radiation osteodystrophy has been regarded as a preneoplastic lesion that evolves into bone tumors (Pool, Hilliams, and Goldman 1973; Pool, Morgan, and Parks 1983; Nilsson, Morgan, and Book 1985). In other studies, radiation osteodystrophy was rarely seen, although bone tumor incidence was high (Gillett et al. 1987).

\section{Osteosarcoma}

\section{Poorly Differentiated Osteosarcoma}

This tumor is characterized by anaplastic mesenchymal cells that range in appearance from small cells, resembling the reticular cell of bone marrow stroma, to the large pleomorphic cells of undifferentiated sarcoma (Figure 1.5). Small amounts of tumor osteoid are present; small tumor bone spicules may also be present. Because of the paucity of matrix production, these tumors form a very lytic radiographic pattern. Secondary pathologic fractures are not uncommon.

\section{Osteoblastic Osteosarcoma}

These tumors are composed of anaplastic osteoblasts and osteogenic precursor cells. The cells are angular, with eccentric hyperchromatic nuclei. The tumors are further subclassified on the basis of the amount of tumor/bone matrix present within the tumor mass. Subclassification is based primarily on histologic examination; however, radiographs are an important ancillary aid that should be used to assess the amount and degree of ossification of tumor/bone matrix present. 


\section{Nonproductive Osteoblastic Osteosarcoma}

This tumor subtype is characterized by the scant amount of tumor osteoid and tumor bone (Figures $1.6 ; 1.7$ ). The radiographic pattern is predominantly lytic in bone, with surrounding soft tissue enlargement. In contrast to the poorly differentiated osteosarcoma, which is the primary differential diagnosis for this tumor type, the cells forming the reproductive osteoblastic osteosarcoma are distinctly osteoblasts.

\section{Moderately Productive Osteoblastic Osteosarcoma}

An intermediate amount of tumor bone and tumor osteoid is present in this tumor subtype (Figure 1.8) Within an individual tumor, either tumor osteoid or tumor bone may be the predominant matrix. Radiographically, a mixed pattern of destruction and production is characteristic of this subtype.

\section{Productive Osteoblastic Osteosarcoma}

Extensive matrix production is evident within tumors of this subtype (Figure 1.9). Abundant, often well-differentiated tumor bone is frequently present. Radiographically, these tumors are very productive and may appear to be sclerotic (Figure 1.10). In the beagle life-span studies, radiation osteodystrophy was often contiguous with tumors of this subtype, and often, no clear distinction between neoplastic and dysplastic bone was apparent.

\section{Chondroblastic Osteosarcoma}

The key feature of this osteosarcoma subtype is that the malignant cells directly produce both tumor bone matrix and tumor cartilage matrix (Figure 1.11). This is in contrast to a chondrosarcoma, in which bone spicules may be present, but the bone spicules are formed by endochondral ossification of the neoplastic cartilage matrix. Because of the mixture of matrix patterns present, these tumors have a mixed pattern on radiographic examination (Figure 1.12).

\section{Fibroblastic Osteosarcoma}

Tumors of this subtype have regions of a spindle cell population with variable degrees of matrix production, thus resembling a fibrosarcoma (Figure 1.13). In addition, however, neoplastic cells can be identified that are directly producing osteoid or bone matrix. The radiographic appearance of this tumor is dependent upon the relative amount of fibroblastic regions, as well as the amount and degree of mineralization of the neoplastic osteoid. In general, the radiographic appearance is that of a lytic bone lesion.

\section{Telangiectatic Osteosarcoma}

Typically this tumor subtype is identified by large, bloody, cystic lesions that are apparent on gross examination, and are identical to the gross lesion produced by a hemangiosarcoma (Figure 1.14). On histologic examination, neoplastic cells, which are usually spindle-shaped, line large, blood-filled cystic spaces within the tumor mass. In addition, tumor osteoid and bone matrix are produced by the same population of neoplastic cells. As one would expect, this tumor has a very osteolytic pattern radiographically. Another histologic pattern for telangiectatic osteosarcomas was identified in beagles exposed by inhalation to ${ }^{90} \mathrm{SrCl}_{2}$ (Gillett et al. 1987). In these tumors, neoplastic spindle-shaped cells formed small capillary spaces in addition to producing tumor bone matrix (Figure 1.15). The vascular component was primarily identified by the presence of the neoplastic cells lining capillary-like structures; however, large, blood-filled cystic spaces were rarely the distinguishing feature of these tumors. 


\section{Giant Cell Type Osteosarcoma}

This tumor subtype is characterized by the presence of numerous tumor giant cells (Figure 1.16). Osteoid and tumor bone matrix are frequently minimal, and, in general, the tumor resembles the nonproductive osteoblastic osteosarcoma. A lytic bone lesion is present radiographically. This tumor subtype occurred very rarely in the beagle life-span studies.

\section{Combined-Type Osteosarcoma}

If more than two of the matrix patterns described above are present, the osteosarcoma is classified as a combined-type osteosarcoma (Figure 1.17). Within the beagle life-span studies, this diagnosis was common and usually resulted from the presence of fibroblastic, osteoblastic, and chondroblastic matrix within the neoplastic mass. The majority of these tumors produce a mixed radiographic pattern (Figure 1.18).

\section{Chondrosarcoma}

The distinguishing feature of a chondrosarcoma is the production of varying amounts of chondroid and fibrillar matrix by the neoplastic cells (Figure 1.19). An important criterion is that the sarcomatous cells never directly produce neoplastic osteoid or bone; this is a feature seen only with osteosarcomas (see chondroblastic osteosarcoma, above). If bone is present in a chondrosarcoma, it is formed secondarily as a result of endochondral ossification following resorption of the tumor matrix. Histologically the tumor is characterized by abnormal cartilage cells, with plump, atypical, hyperchromatic nuclei. Mitotic figures may or may not be present. Binucleate or multinucleated cells, or the presence of lacunae containing more than one cartilage cell, are frequently the only morphologic indication of cell division (Figure 1.20). The amount of chondroid matrix produced by different chondrosarcomas is frequently quite variable. When the matrix becomes secondarily fibrillar and hyalinized, morphologically resembling osteoid, or when endochondral ossification occurs, it may be difficult to distinguish the chondrosarcoma from an osteosarcoma.

The radiographic appearance of a chondrosarcoma is frequently variable, and, often, a chondrosarcoma cannot be differentiated from an osteosarcoma (Figure 1.21).

\section{Fibrosarcoma}

Fibrosarcoma of bone is a malignant neoplasm of the fibrous connective tissue, originating from stromal elements (Figures $1.22 ; 1.24$ ). A variable amount of collagenous matrix is produced, but neither neoplastic bone nor cartilage is present. It may be difficult to differentiate this tumor from a fibroblastic osteosarcoma of low osteogenic potential. As would be anticipated, these tumors produce a lytic radiographic lesion, although in some instances only soft-tissue swelling is found. Usually a fibrosarcoma evokes a milder, periosteal response than that observed with an osteosarcoma. Histologically, the tumors resemble fibrosarcomas occurring elsewhere in the body. The majority of these tumors are well-differentiated; occasionally, the tumor may be anaplastic and highly cellular, making it difficult to distinguish it from a poorly differentiated osteosarcoma or nonproductive osteoblastic osteosarcoma. Using special stains, neither cross-striations in the cytoplasms nor evidence of alkaline phosphatase activity can be identified in this neoplasm.

\section{Hemangiosarcoma}

The hemangiosarcoma in bone is defined as a malignant neoplasm of endothelial cells, arising from the vasculature of a bone organ (Figure 1.25). This tumor type was not infrequent among some of the beagle life-span studies. Because of the very destructive nature of this tumor, secondary pathologic fractures can occur. Radiographically the tumor appears as a highly destructive lesion, often evoking only a minimal degree of extracortical reactive bone. Histologic characteristics of this 
tumor are the same as those observed for a hemangiosarcoma arising in soft tissue. The characteristic feature of this tumor is the formation of vascular channels and spaces lined by neoplastic endothelial cells. The channels may coalesce and enlarge to resemble a cavernous structure. The stroma of a hemangiosarcoma does not form a calcifiable matrix. Telangiectatic osteosarcomas, in which osteoid formation is minimal, may be difficult to distinguish from a primary hemangiosarcoma, particularly if only a small piece of tissue is available for examination.

The remaining bone tumors listed in Table 1.1 (giant cell tumor of bone, multilobular tumor, liposarcoma, and lymphoid and myelomatous tumors) were not identified in beagles exposed to boneseeking radionuclides. The distinguishing characteristics of these remaining tumors are described by Pool, Hilliams, and Goldman (1973) and Pool (1990).

\section{REFERENCES}

Gillett, N. A., B. A. Muggenburg, B. B. Boecker, H. C. Griffith, F. F. Hahn, and R. O. McClellan. 1987. Single inhalation exposure to ${ }^{90} \mathrm{SrCl}_{2}$ in the Beagle dog: late biological effects. J. Natl. Cancer Inst. 79:359-376.

Hahn, F. F., J. A. Mewhinney, B. S. Merickel, R. A. Guilmette, B. B. Boecker, and R. O. McClellan. 1981. Primary bone neoplasms in Beagle dogs exposed by inhalation to aerosols of plutonium-238 dioxide. J. Natl. Cancer Inst. 67:917-927.

Nilsson, A., J. P. Morgan, and S. Book. 1985. Investigations of ${ }^{90} \mathrm{SrCl}_{2}$ in dogs. I. Pathogenesis of radiation-induced bone tumors. Acta Radiol. Oncol. 24:95-110.

Pool, R. R, R.J.R. Hilliams, and M. Goldman. 1973. Induction of tumors involving bone in beagles fed toxic levels of strontium 90. Am. J. Roent. Radium Ther. Nucl. Med. 118:900-908.

Pool, R. R., J. P. Morgan, and N. J. Parks. 1983. Comparative pathogenesis of radium-induced intracortical bone lesions in humans and beagles. Health Phys. 44:155-177.

Pool, R. R. 1990. Tumors of bone and cartilage. In: Tumors in Domestic Animals, J. E. Moulton, Ed., 3rd ed. University of California Press, Berkeley, CA. pp. 157-230. 


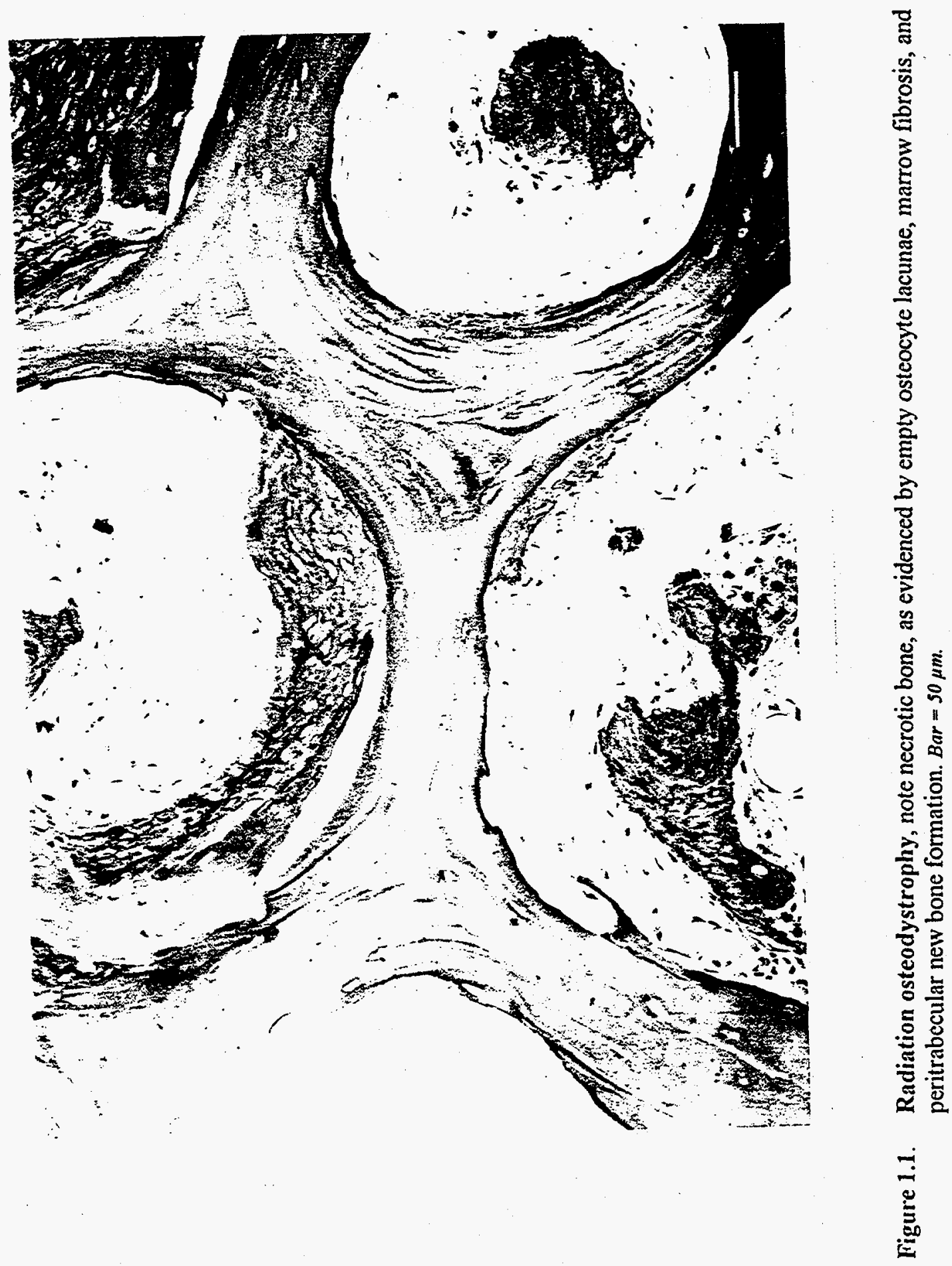




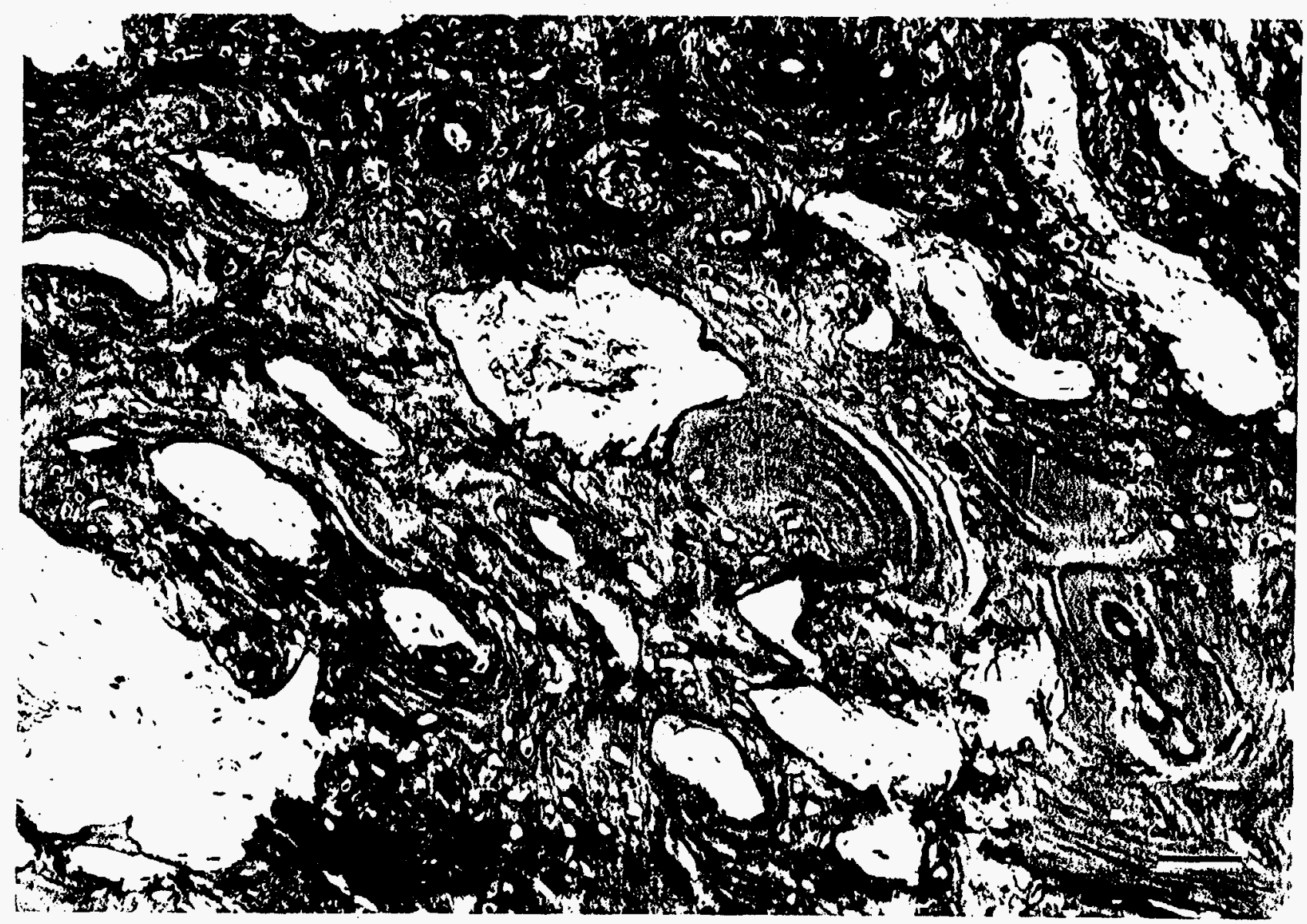

Figure 1.2. Radiation osteodystrophy: example of macroresorptive cavities; marrow fibrosis and abundant deposition of woven bone are evident. $\mathrm{Bar}=50 \mu \mathrm{m}$. 


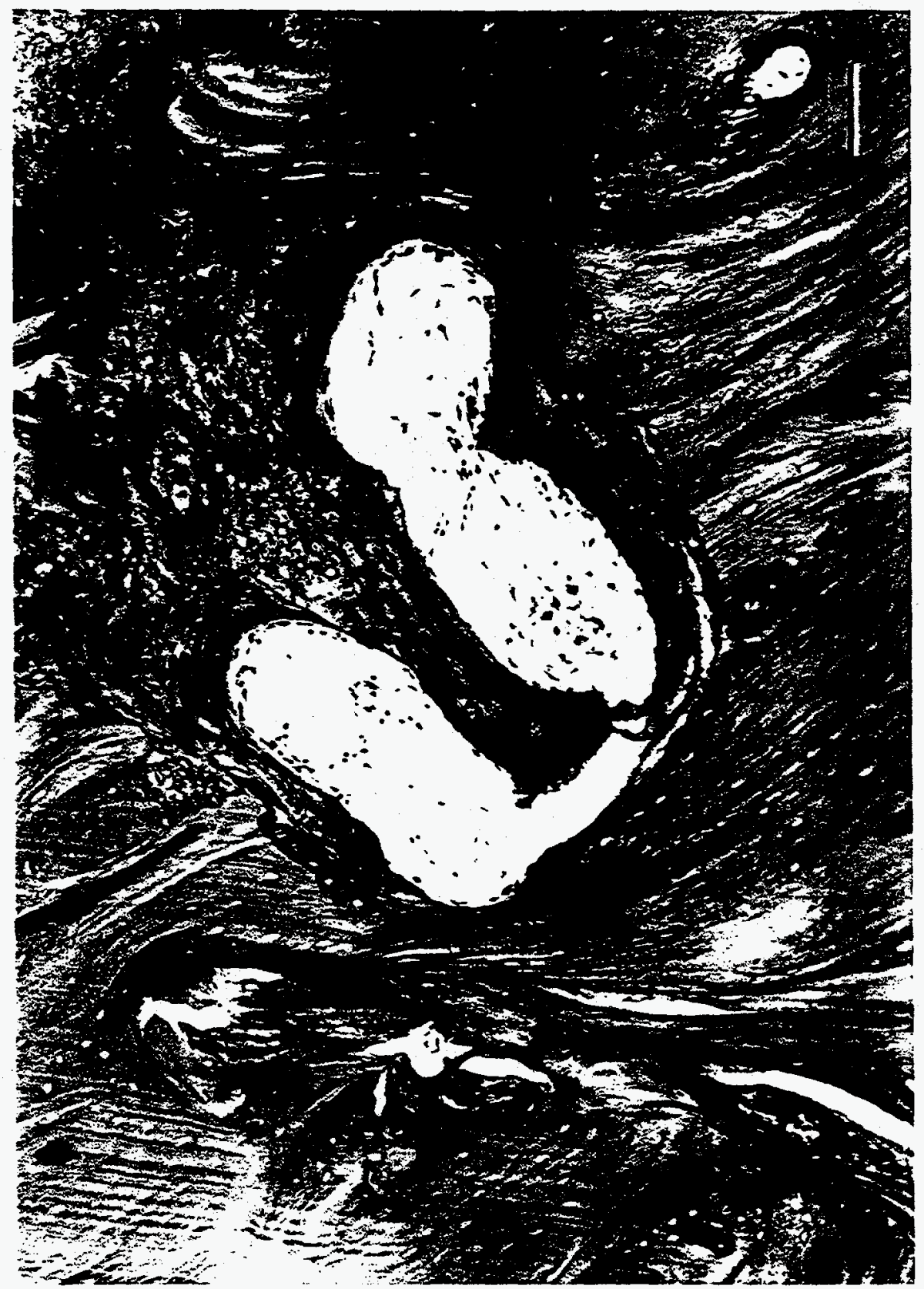


Bone

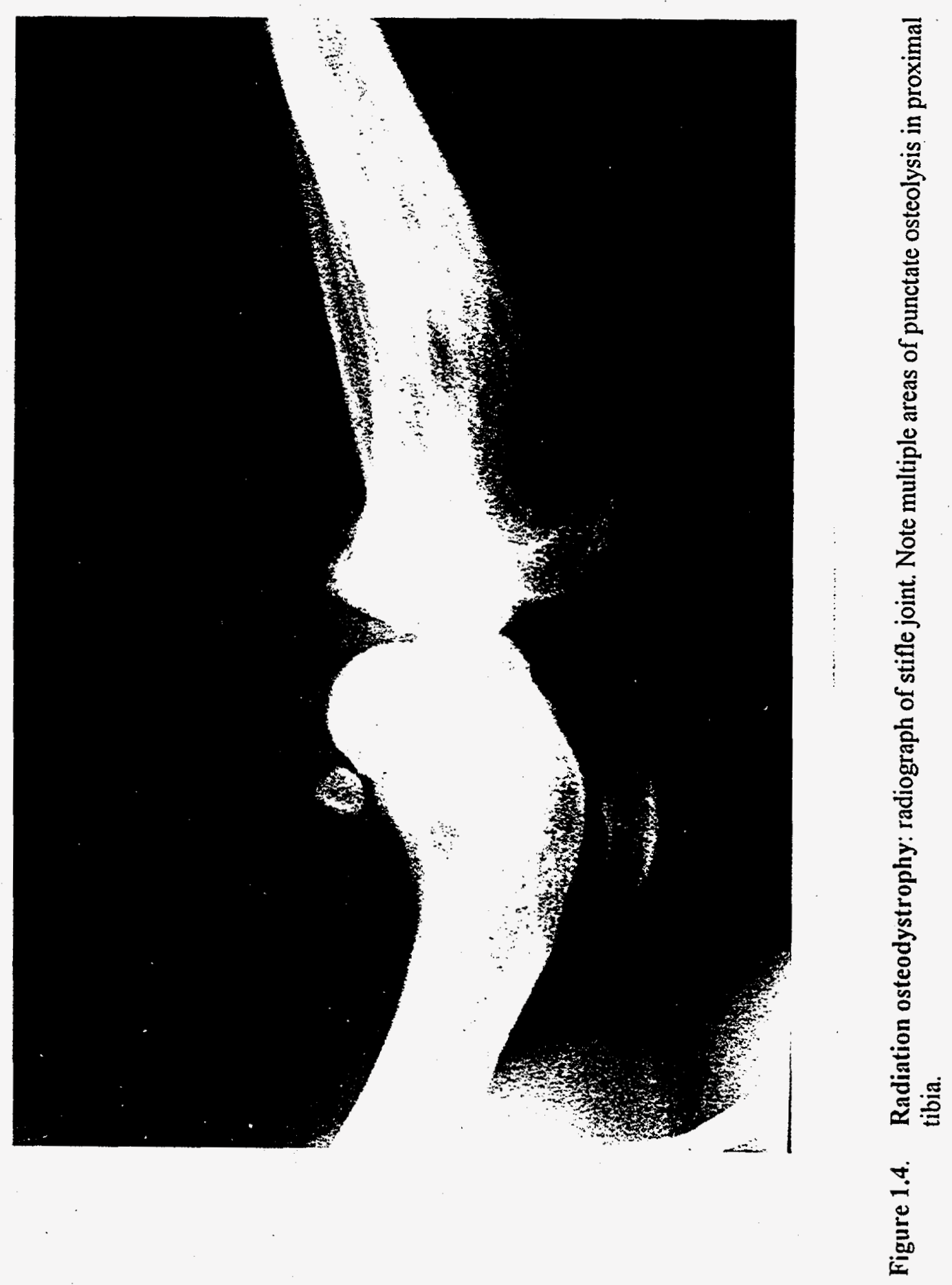




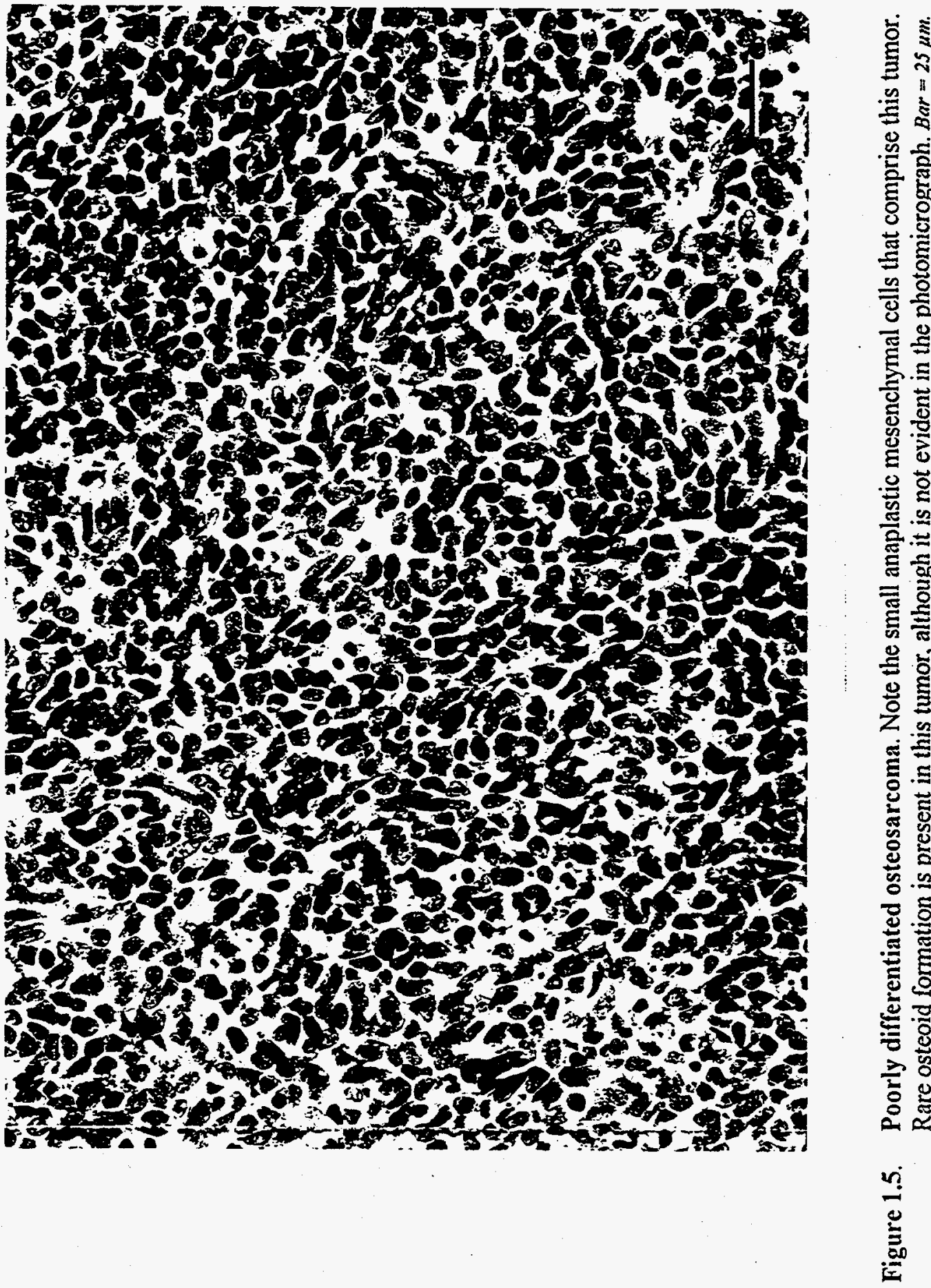




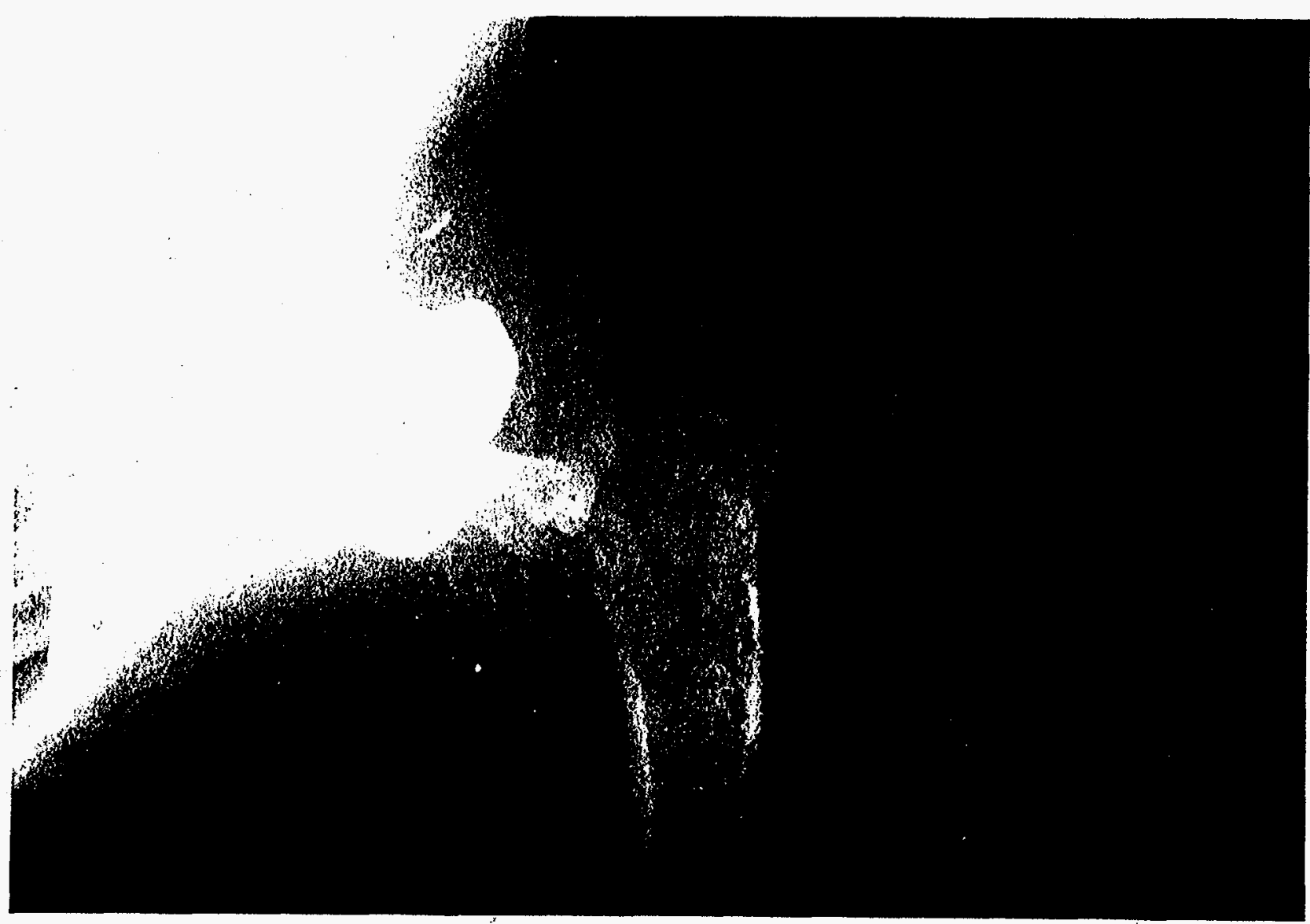

Figure 1.6. Radiograph of nonproductive osteoblastic osteosarcoma. Note extensive osteolysis, pathologic fracture and scant evidence of bone production. 


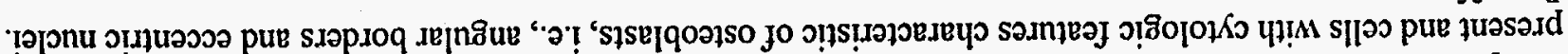

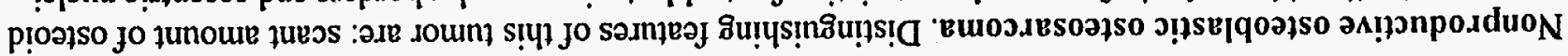

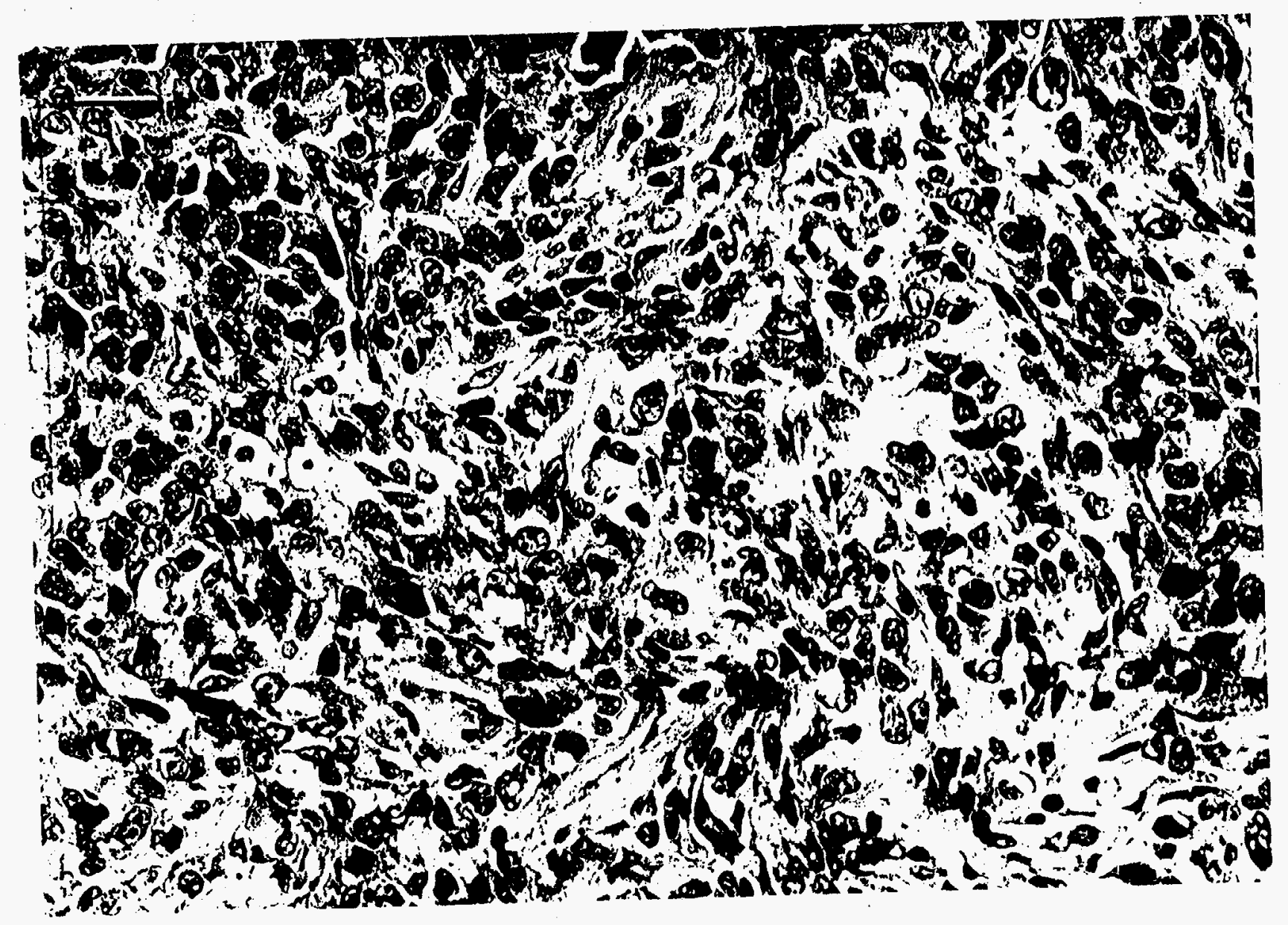




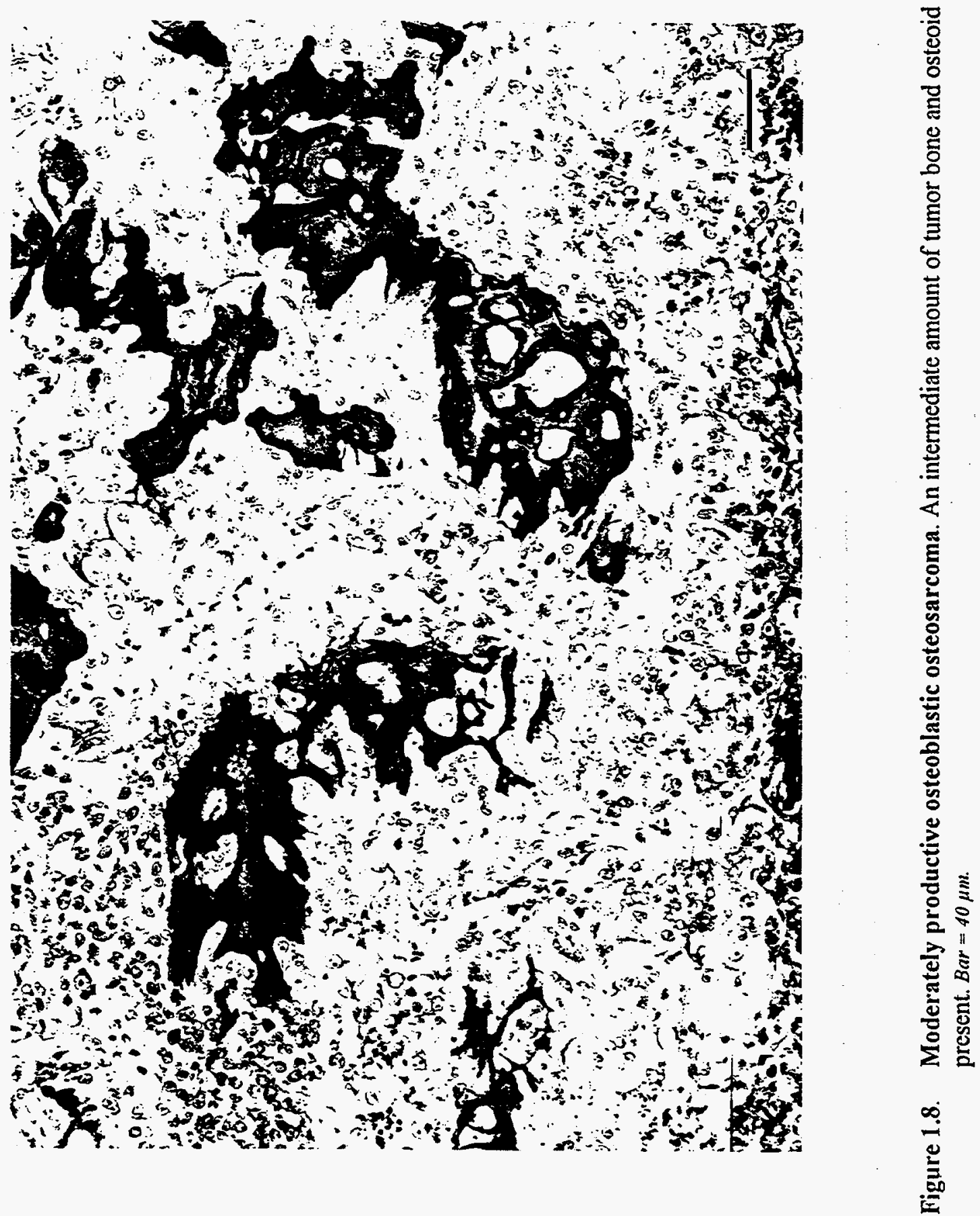




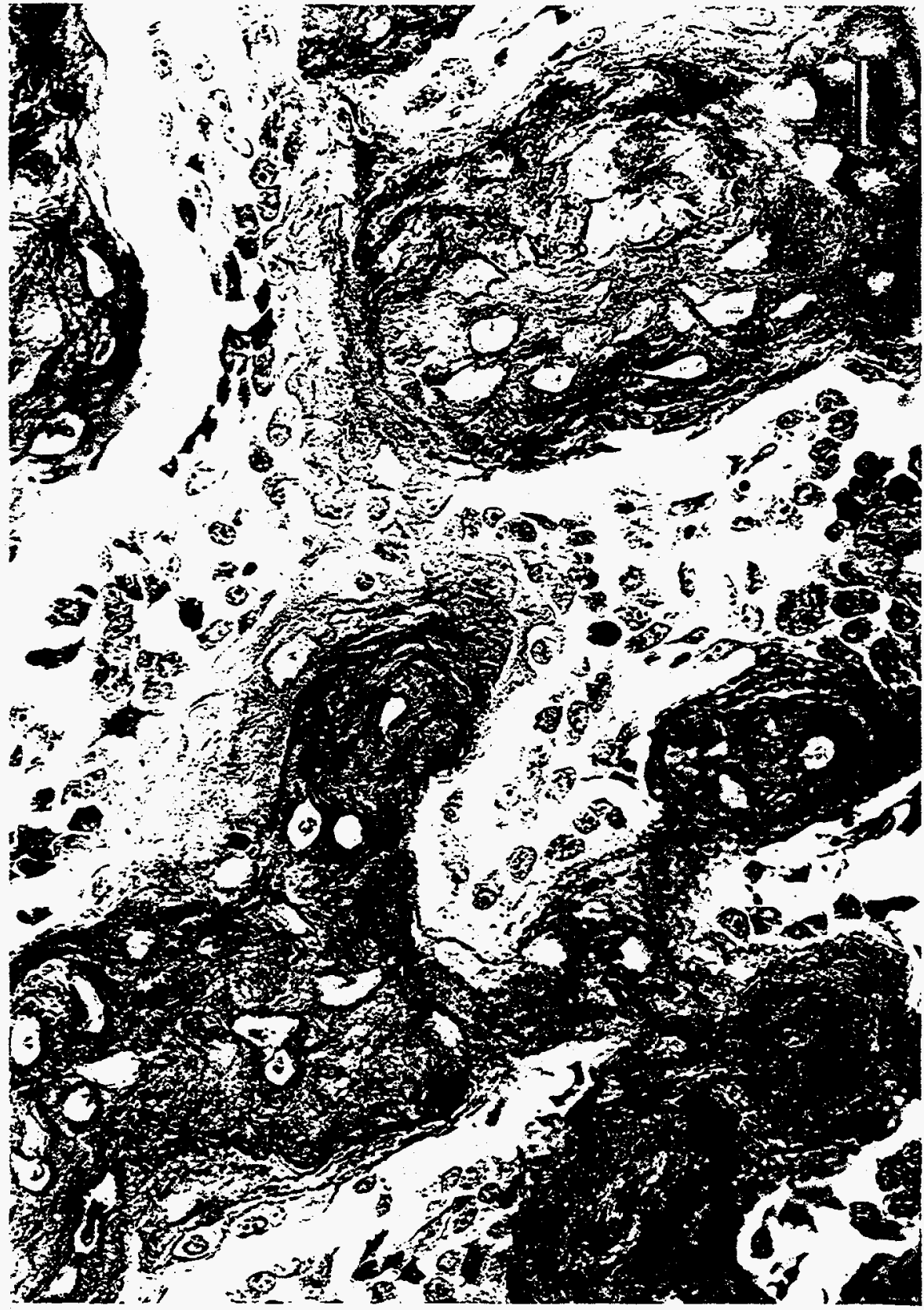

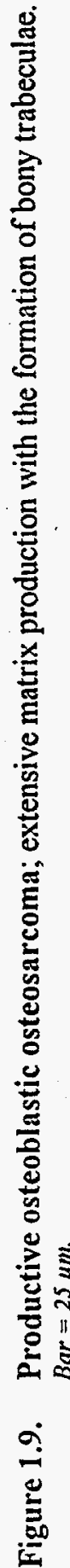




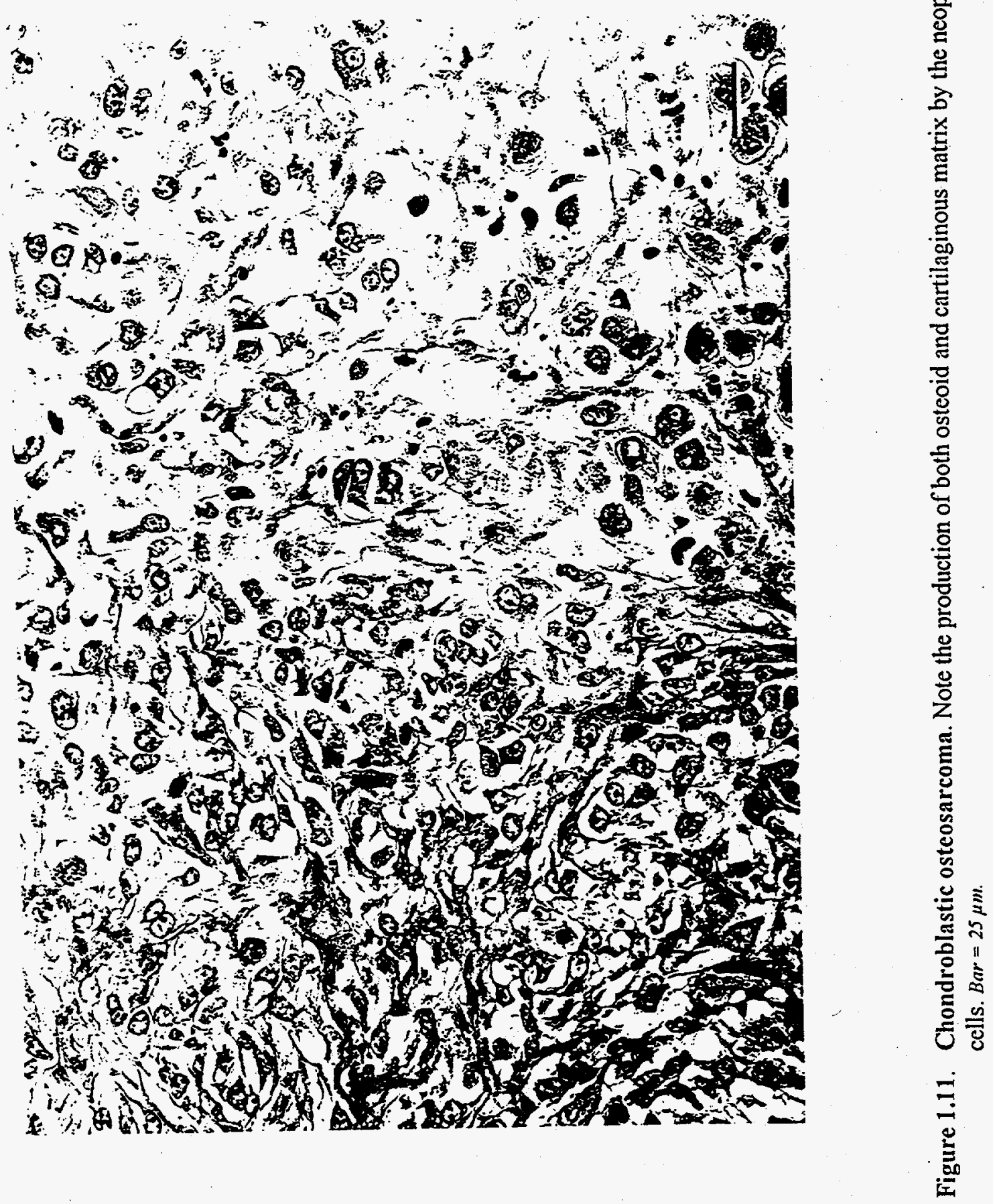




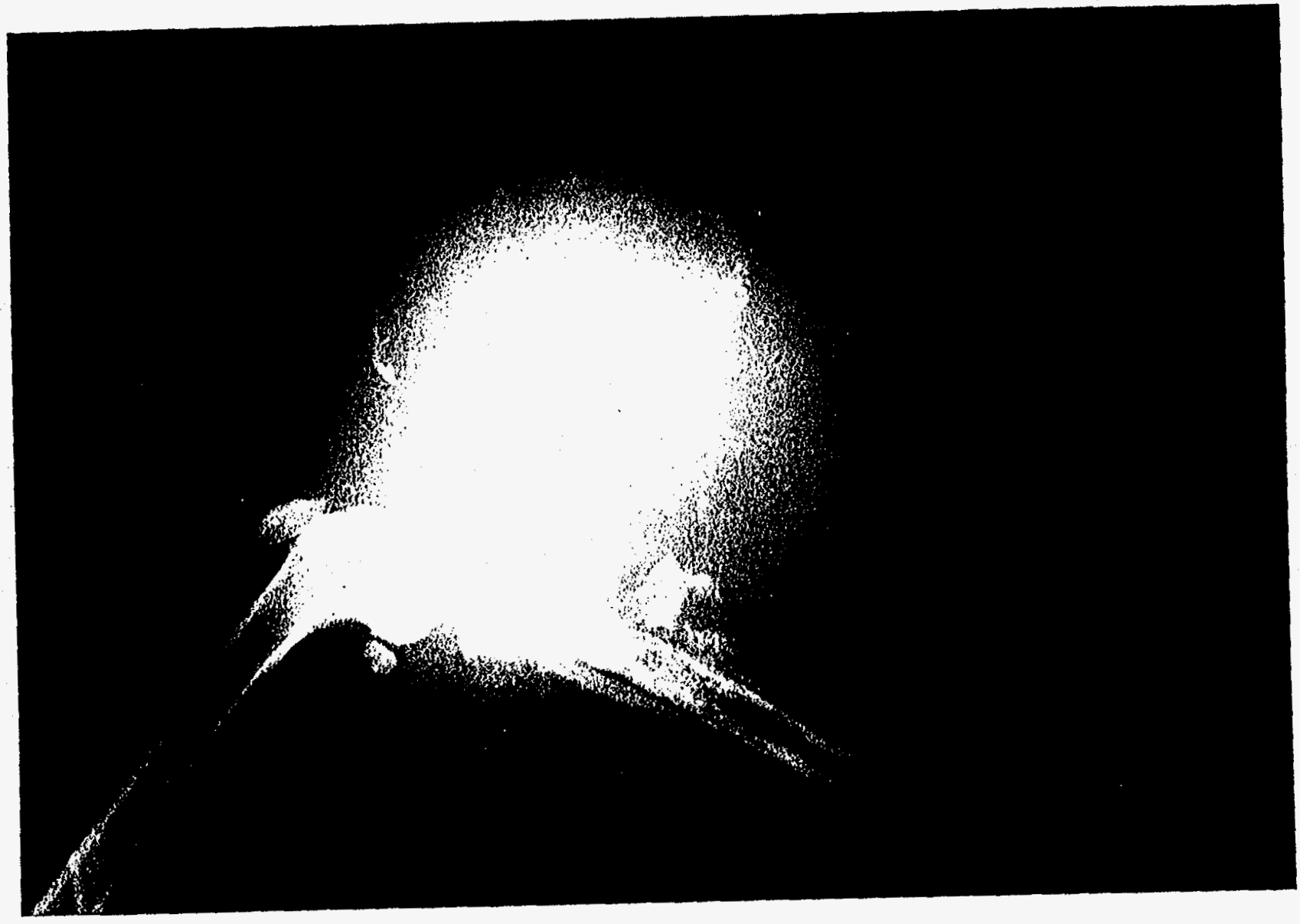

Figure 1.12. Radiograph of chondroblastic osteosarcoma in the proximal tibia. Note the predominantly lytic pattern of tumor growth, in addition to evidence of bone production. 


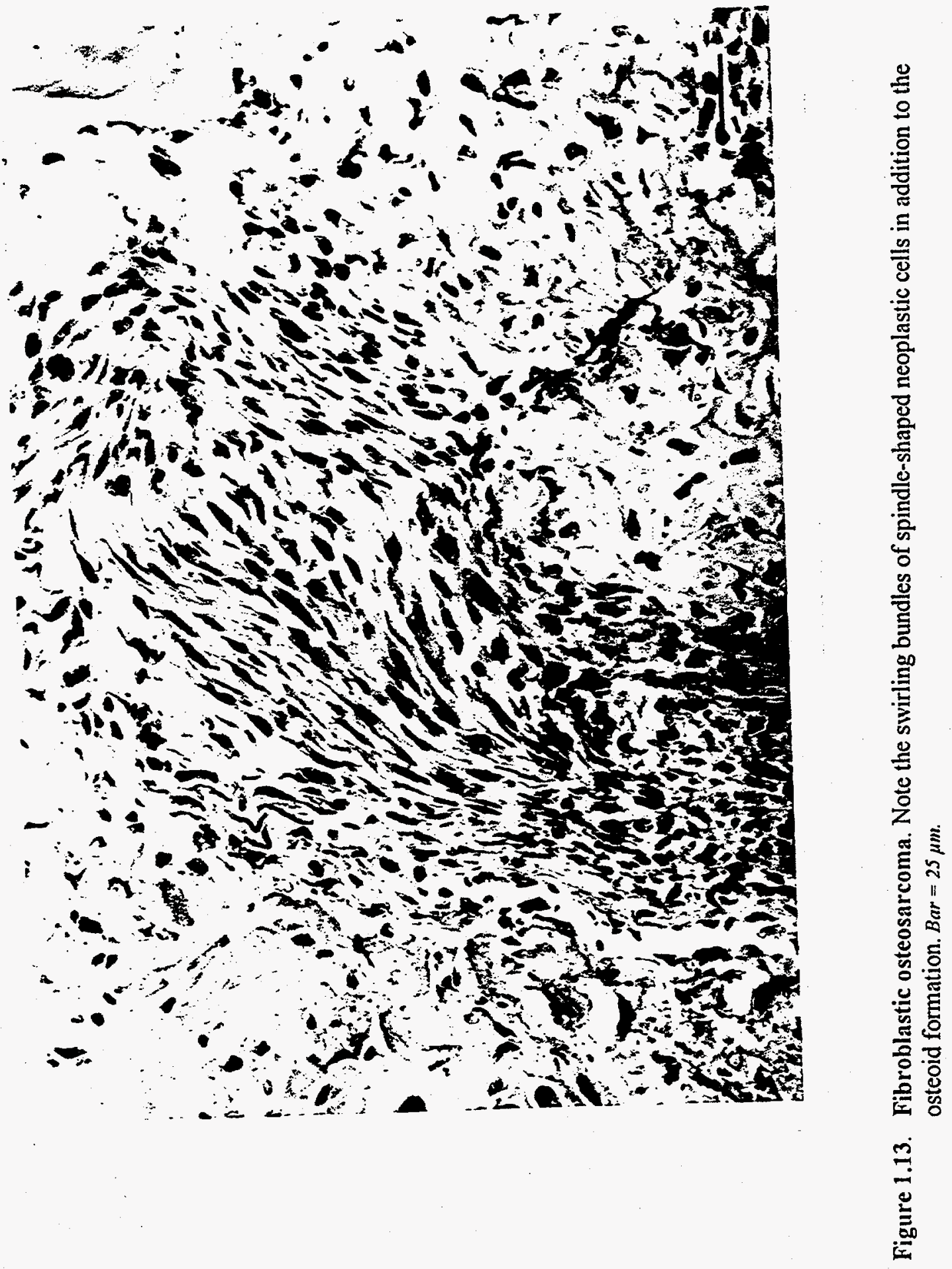




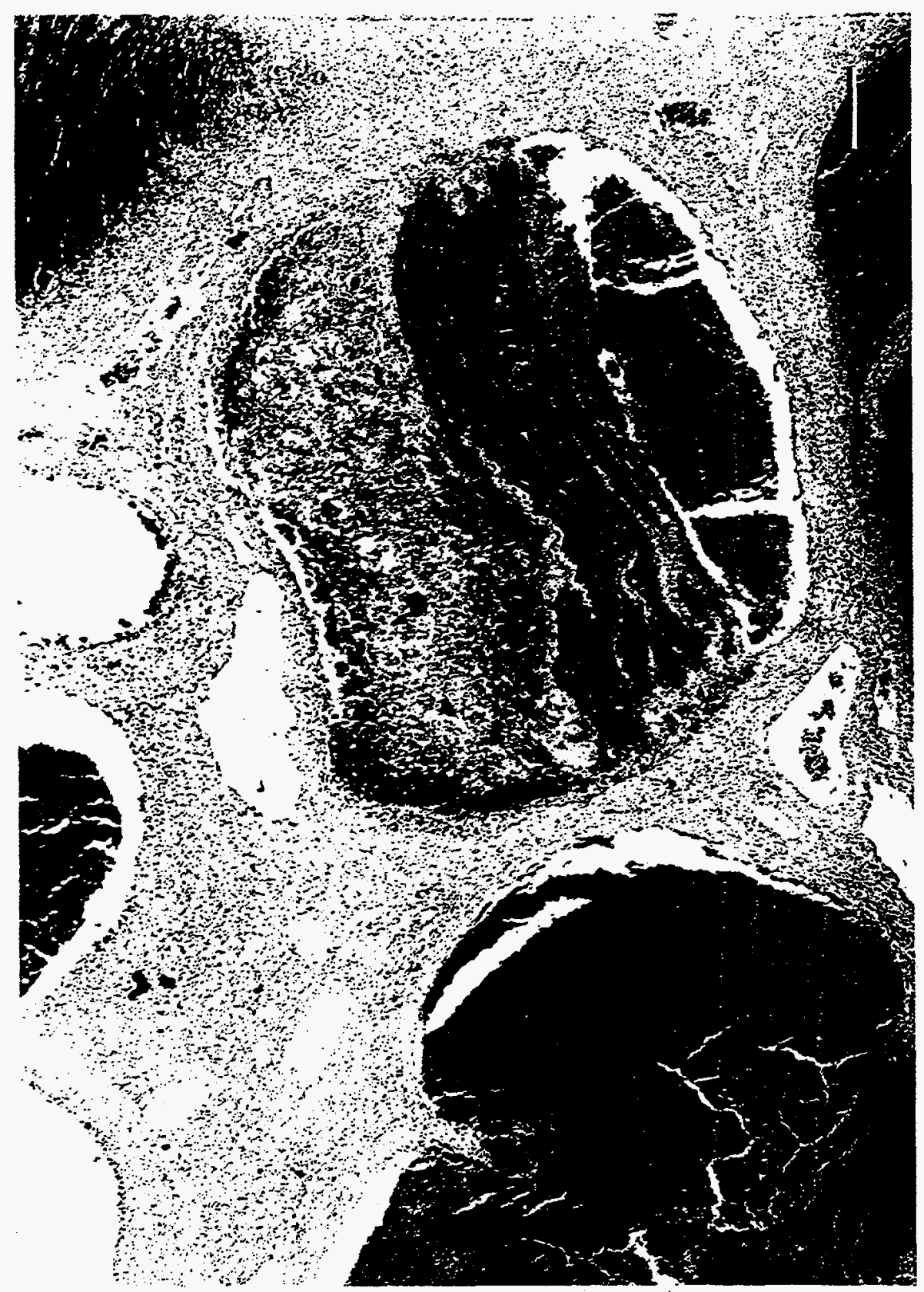

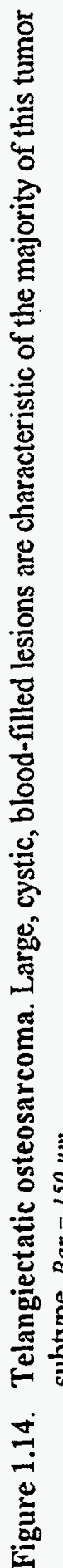




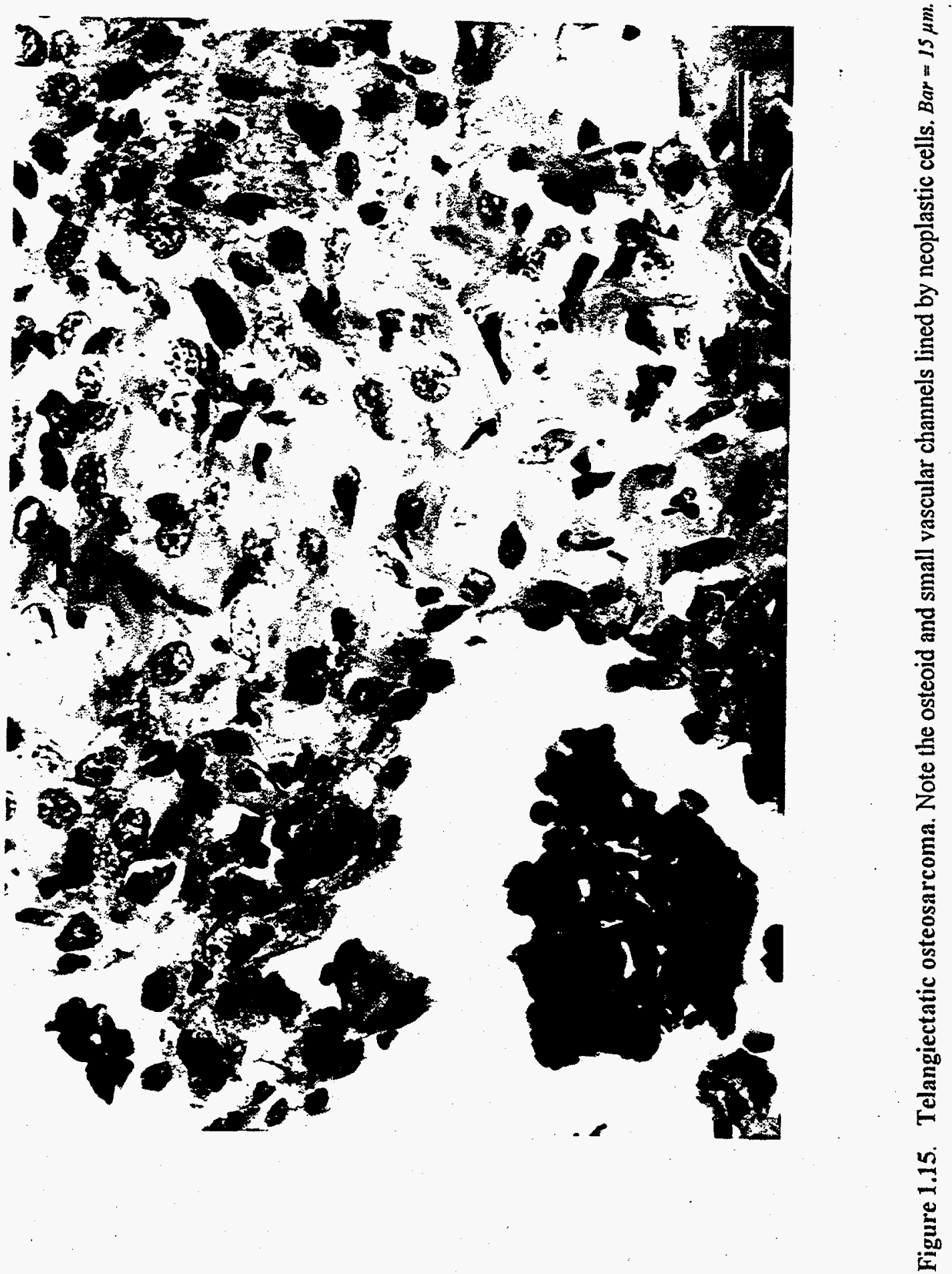




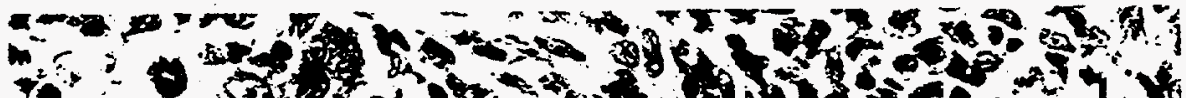

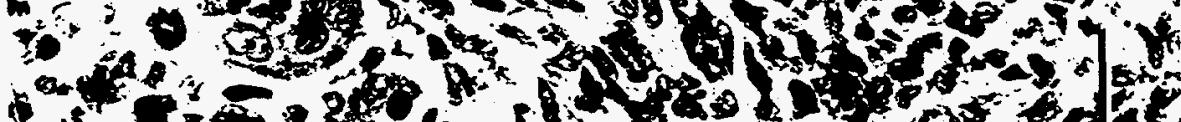

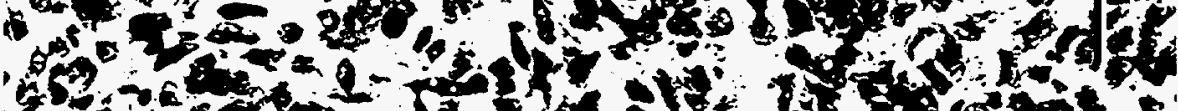

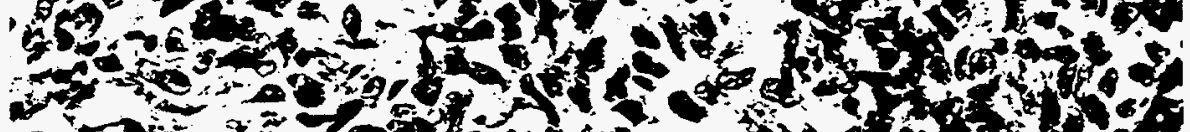

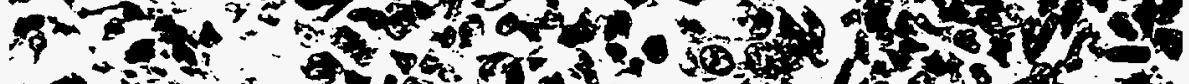

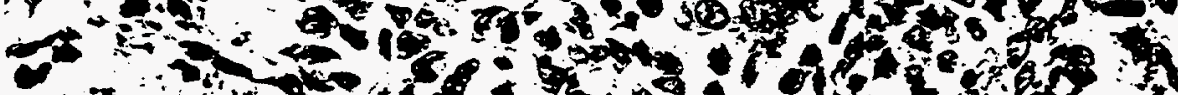

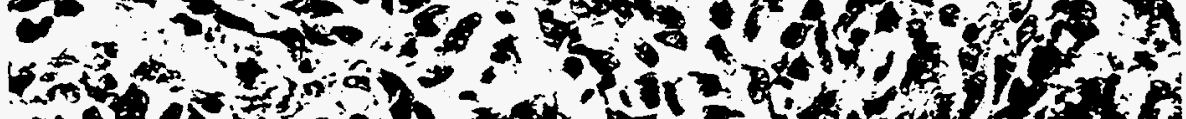

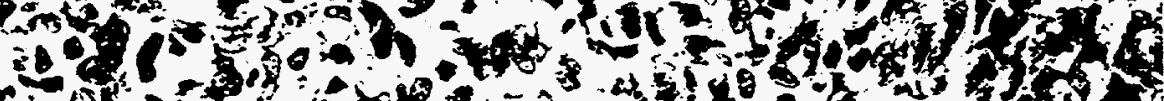

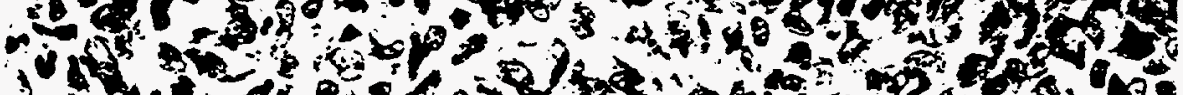

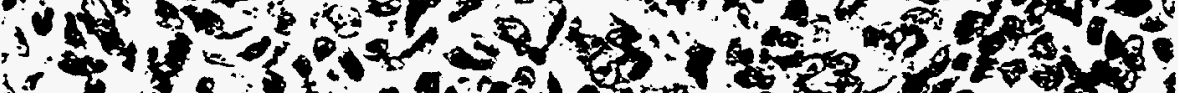

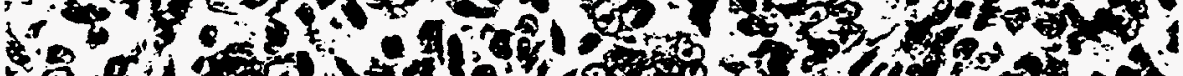

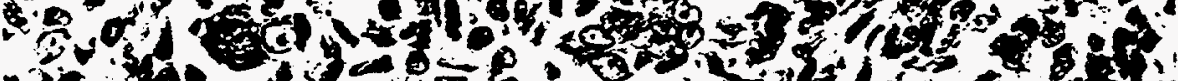

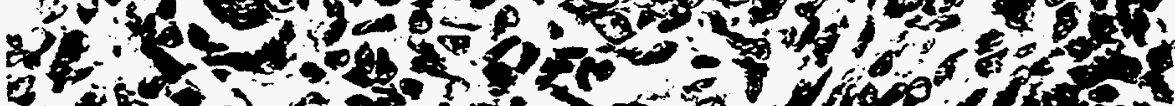
Q 20 -

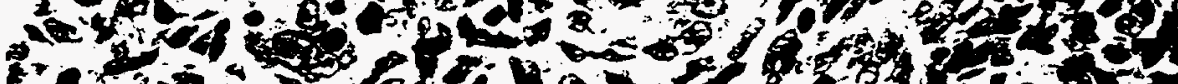

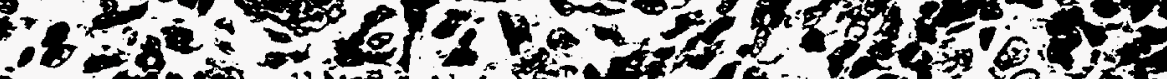

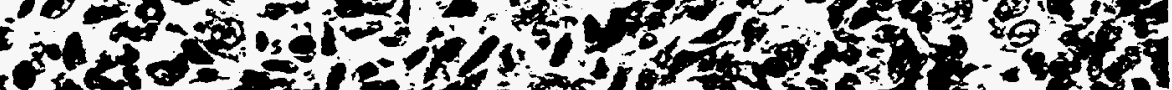

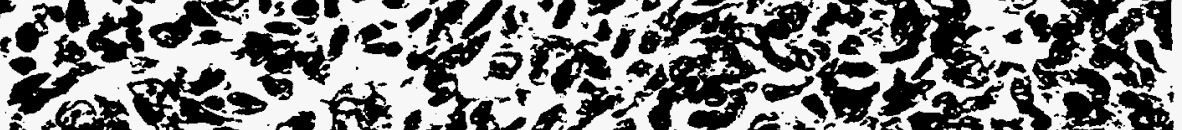

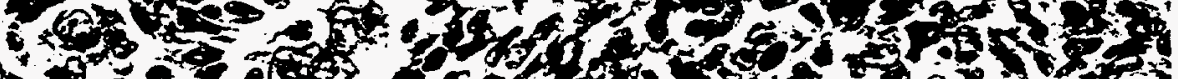

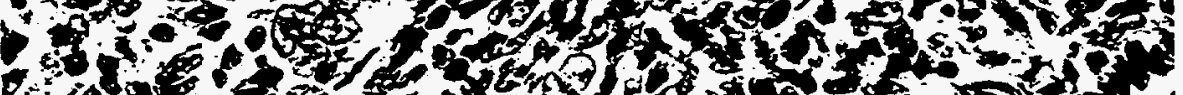

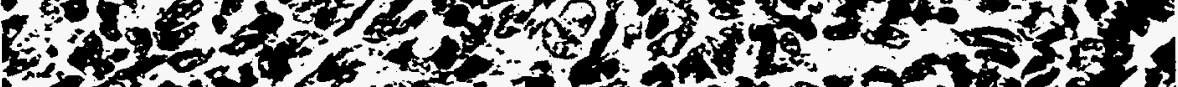

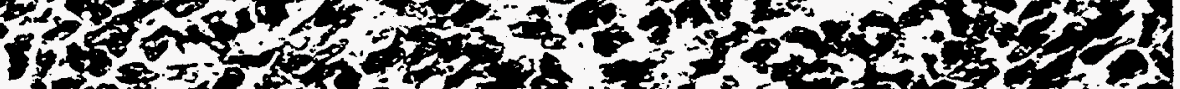

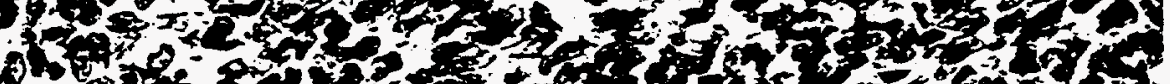

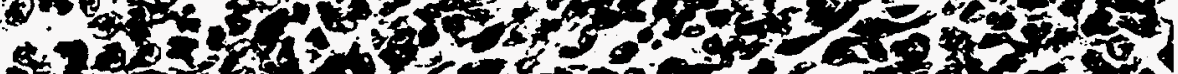

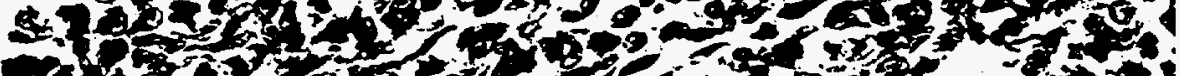

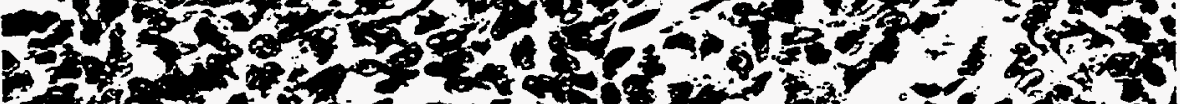

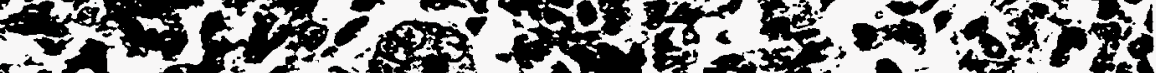
f.J J

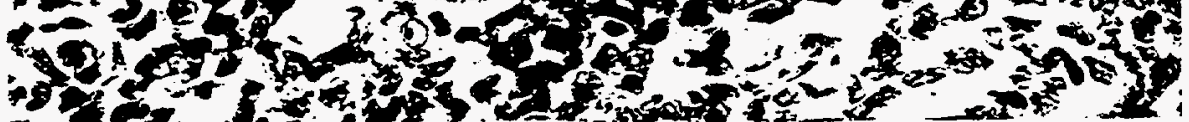




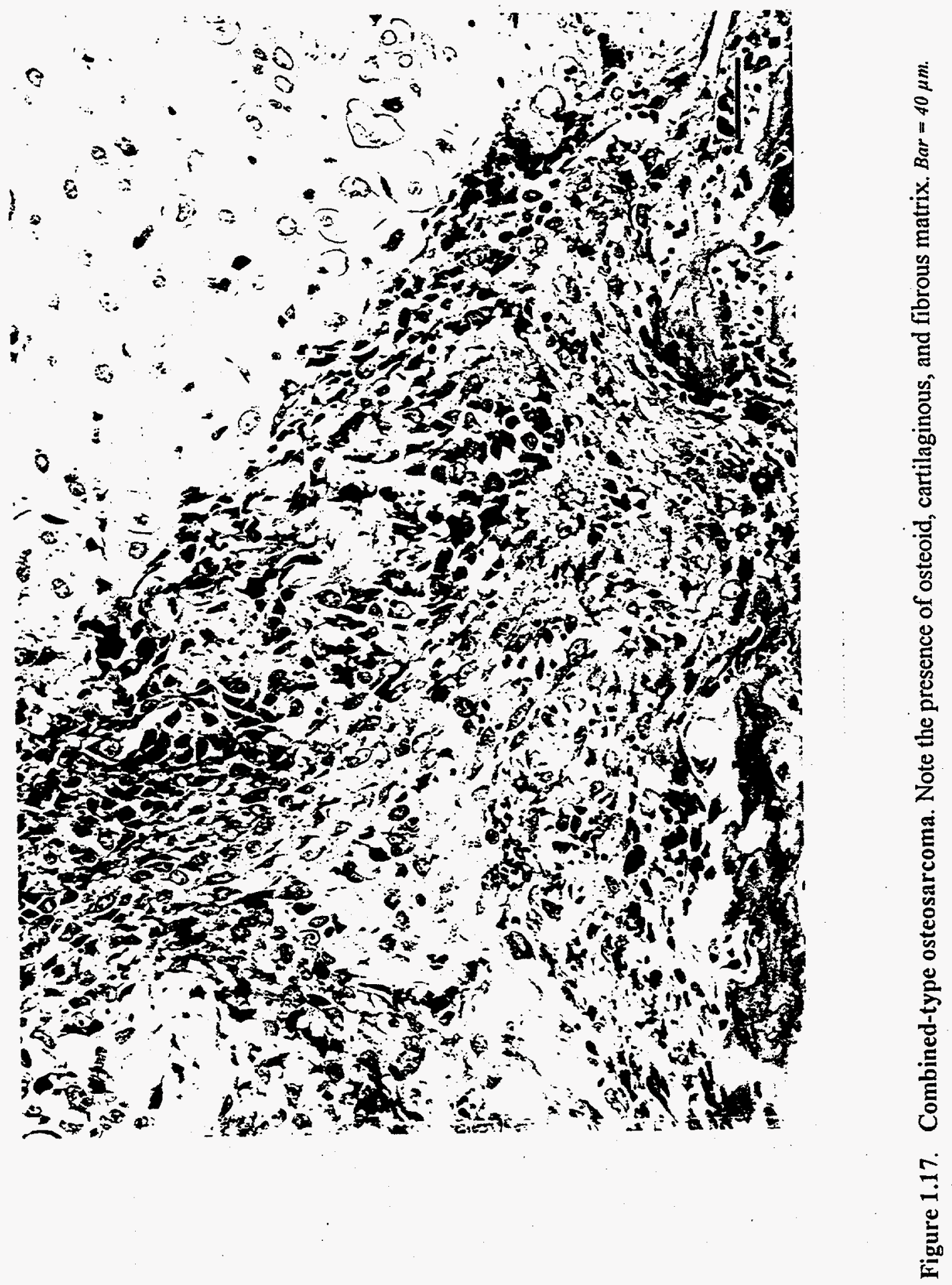


Bone

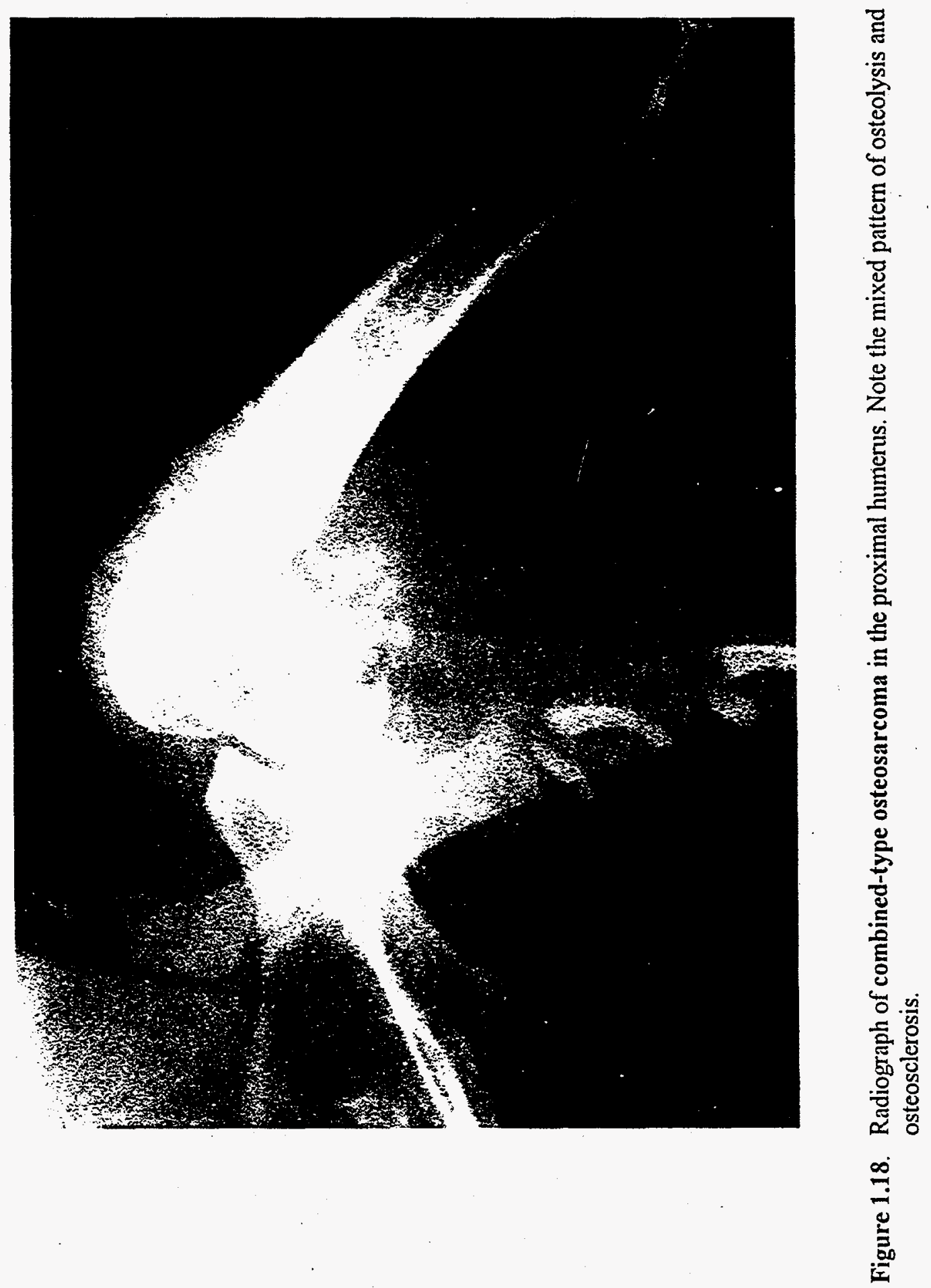


Bone

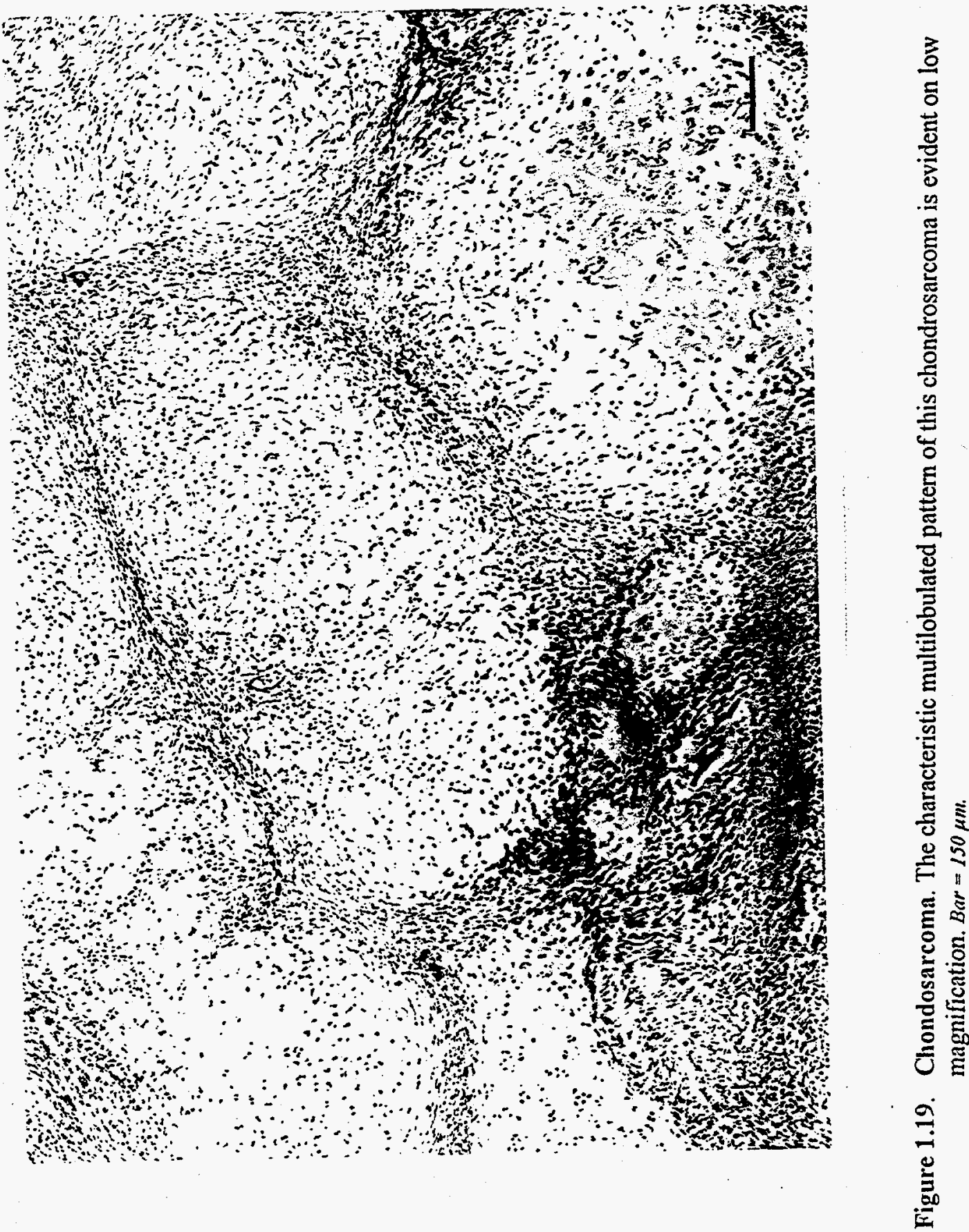


Bone
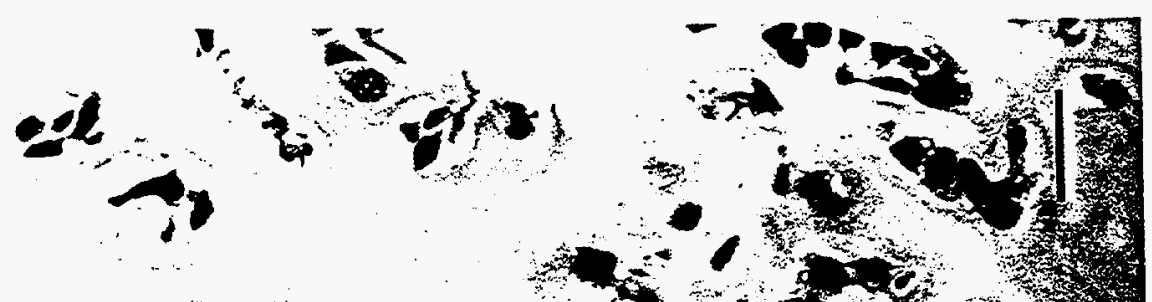

40

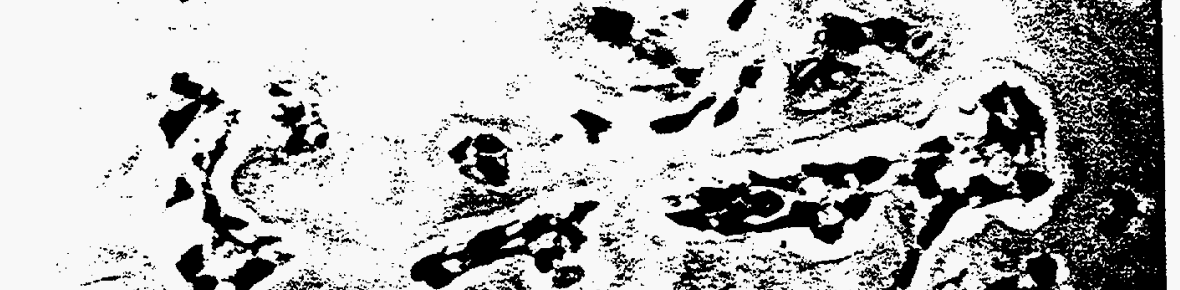

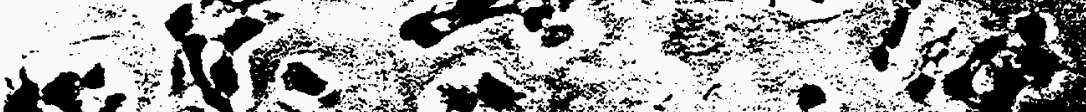

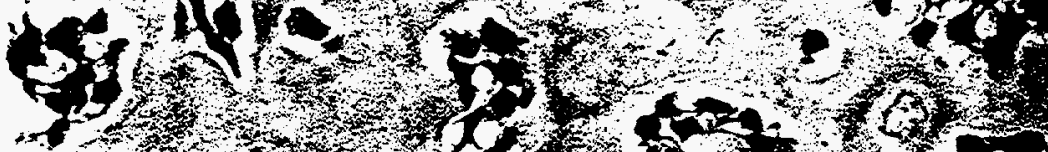

$\$ 1$

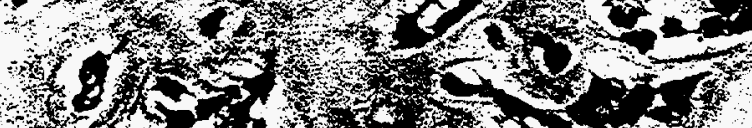

$+3+2$
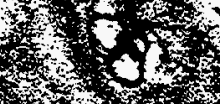

.

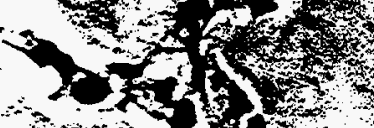

$20,0,0,0$

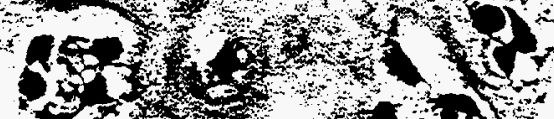

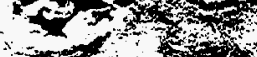

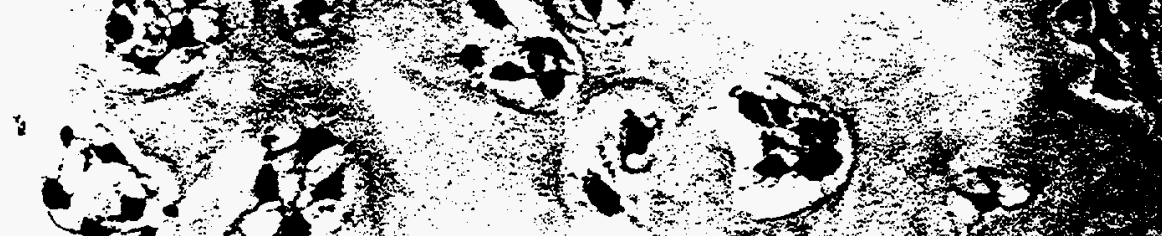

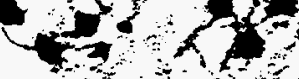

$$
+1
$$

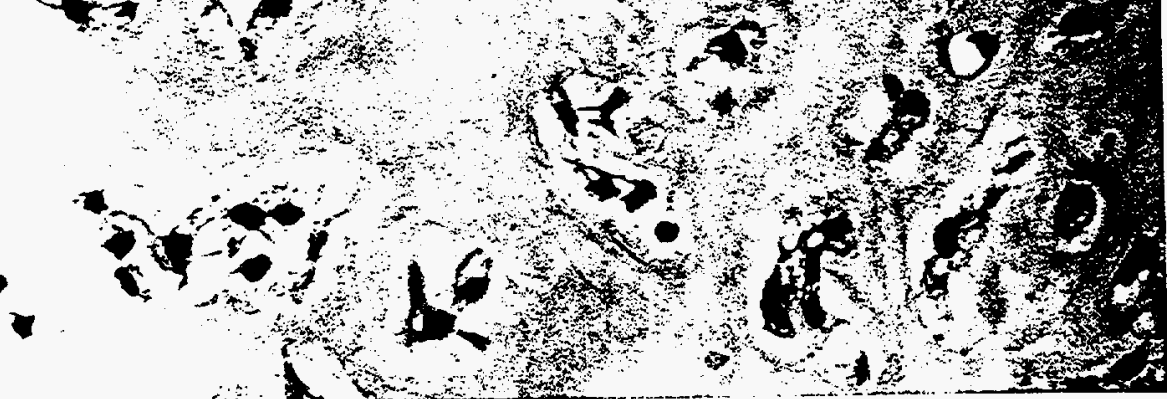




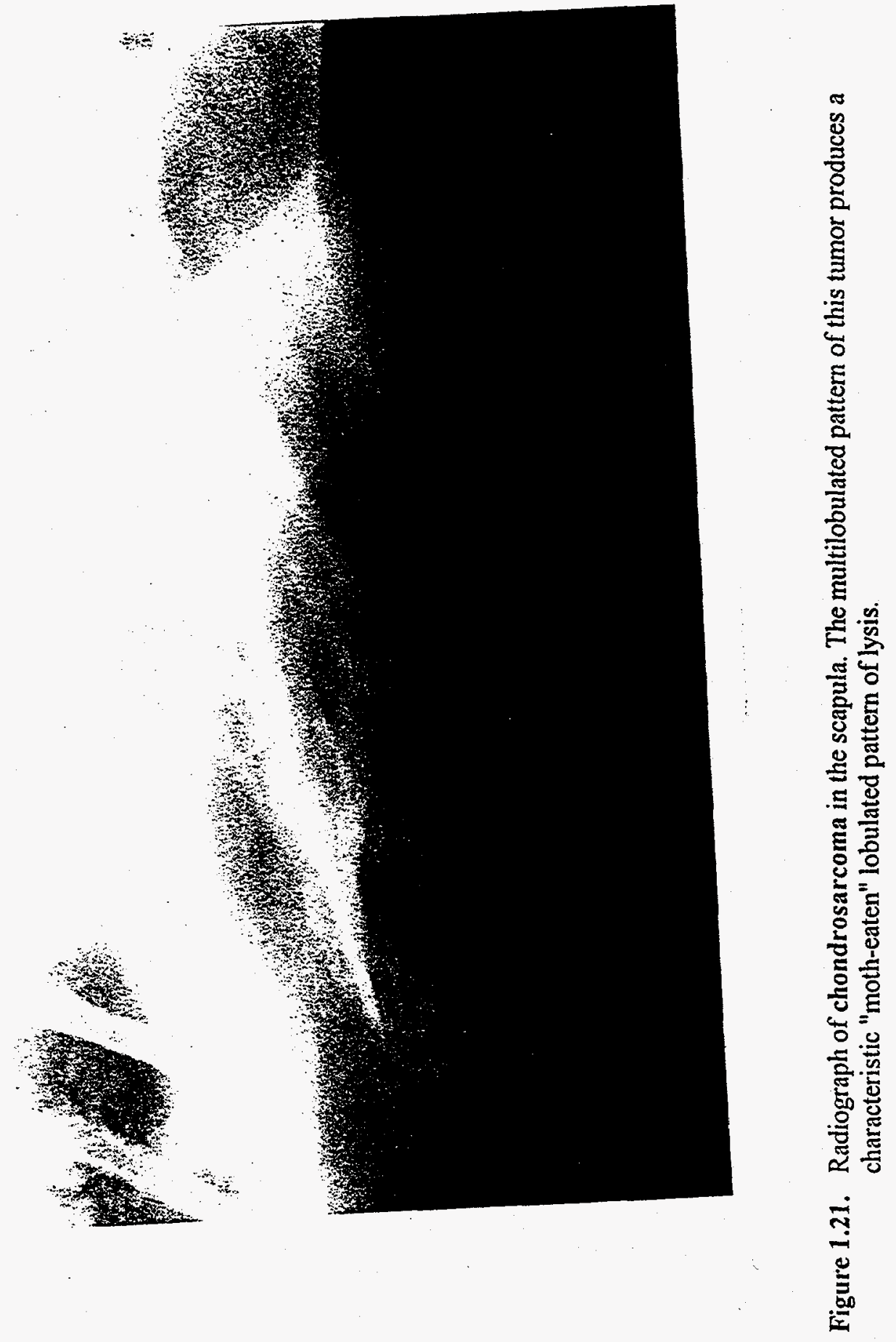




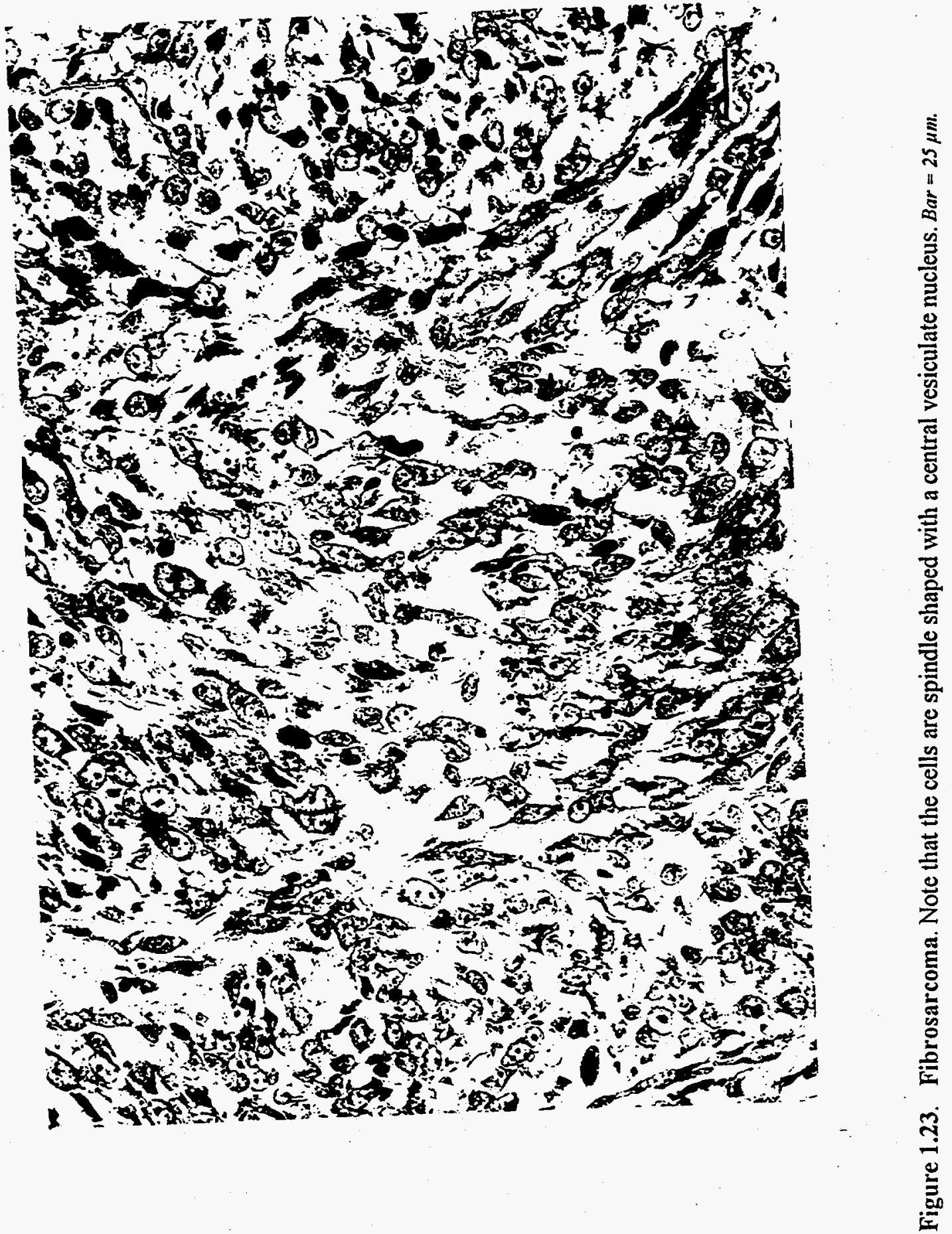




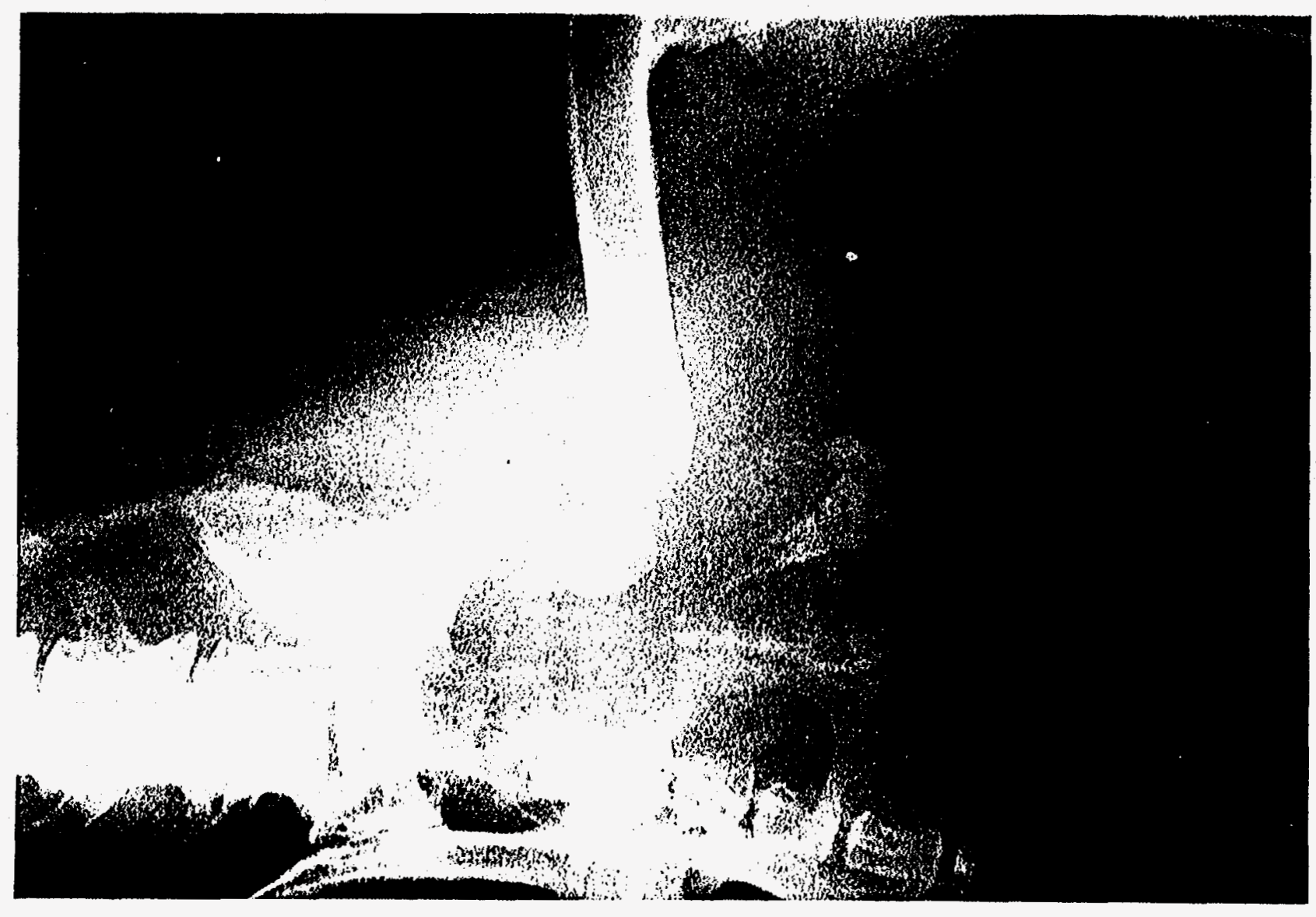

Figure 1.24. Radiograph of a fibrosarcoma. Note that only evidence of tumor is osteolysis of the bone and soft-tissue density. 


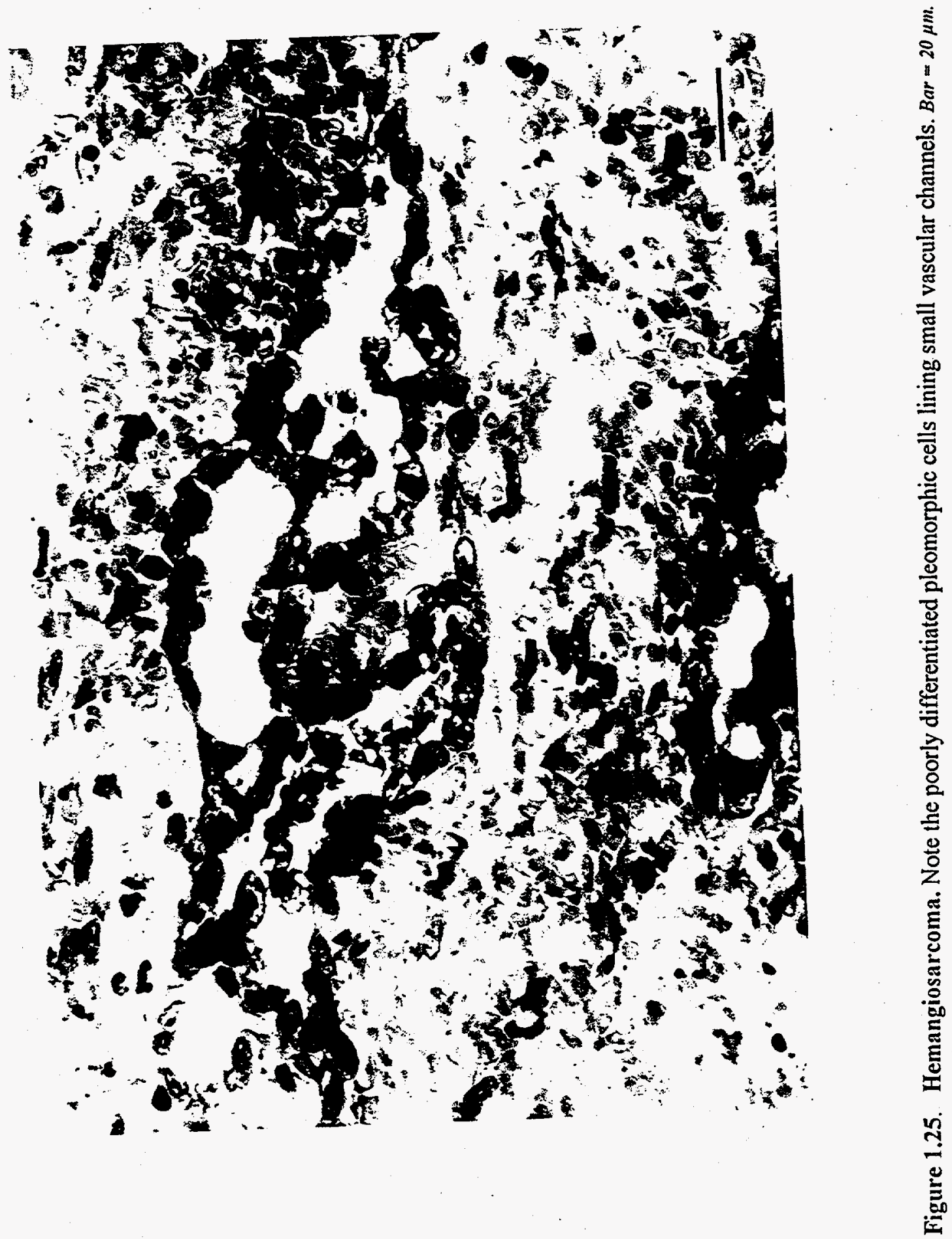


Myeloid Leukemia 


\title{
CHAPTER 2: Radiation-Induced Myeloid Leukemia
}

\author{
David V. Tolle, Thomas E. Fritz, and Thomas M. Seed ${ }^{1}$ \\ Biological and Medical Research Division \\ Argonne National Laboratory \\ ${ }^{1}$ Currently at Armed Forces Radiobiology Research Institute
}

\section{INTRODUCTION}

The classification used in this atlas chapter is based on the French-American-British (FAB) system for the classification of acute myeloid leukemia in humans (Bennett et al., 1976, 1985a,b). We have made some modifications and additions to include variations observed in the canine leukemia complex that are not addressed by the FAB system. We subdivided FAB-M7 (megakaryocytic leukemia), into $\mathrm{M} 7 \mathrm{a}$ (without maturation) and $\mathrm{M} 7 \mathrm{~b}$ (with maturation); and we added erythremic myelosis (EM). The FAB Codes M1-7 and the corresponding Systemized Nomenclature of Medicine (SNOMED) codes are shown in Table 2.1, along with an index to illustrative figures.

Table 2.1. Myeloid Leukemia nomenclature, index of figures, and SNODOG database codes

\begin{tabular}{|c|c|c|c|}
\hline Nomenclature & $\begin{array}{c}\text { FAB/ANL } \\
\text { Classification }\end{array}$ & Figure(s) & $\begin{array}{c}\text { Proposed } \\
\text { SNODOG } \\
\text { code }^{\boldsymbol{a}}\end{array}$ \\
\hline \multicolumn{4}{|l|}{ Neoplastic } \\
\hline Myeloid leukemia without maturation & M1 & $2.1-3$ & M98613\% \\
\hline Myeloid leukemia with maturation & M2 & $2.4-6$ & M986132 \\
\hline Hypergranular promyelocytric leukemia & M3 & Not seen & \\
\hline Myelomonocytic leukemia & M4 & $2.7-9$ & M98603\% \\
\hline Monocytic leukemia without maturation & M5a & $2.10-15$ & M98903/ \\
\hline Monocytic leukemia with maturation & M5b & $2.16-17$ & M989032 \\
\hline Erythroleukemia & M6 & $2.18-22$ & M984030 \\
\hline Erythremic myelosis & EM & $2.23-24$ & M984031 \\
\hline Megakaryocytic leukemia without maturation & M7a & $2.25-26$ & M99103\% \\
\hline Megakaryocytic leukemia with maturation & M7b & $\begin{array}{l}2.29-31 \\
2.34-35\end{array}$ & M991032 \\
\hline \multicolumn{4}{|l|}{ Non-neoplastic } \\
\hline Myelofibrosis & none & $2.32-33$ & M490005 \\
\hline
\end{tabular}

Table 2.2 shows dosimetric and clinical details of the 20 illustrative cases. All cases are cytologically and morphologically identified and typed by examination of (a) blood and bone-marrow films and tissue imprints stained with Wright-Giemsa (W-G), and (b) histologic sections of marrow and other tissues. In addition, in many cases, needle aspirates of liver, spleen, and lymph nodes were collected and are available for study. Cytochemistry, as well as both transmission and scanning electron microscopy, was used where needed to confirm diagnosis. 
Table 2.2. Selected Exposure History, Terminal Hematology, and Organ Weights for 20 Illustrated Cases

\begin{tabular}{|c|c|c|c|c|c|c|c|c|c|}
\hline \multirow[b]{2}{*}{$\begin{array}{c}\text { FAB/ANL } \\
\text { Classiffcation } \\
\text { Incidence }\end{array}$} & \multirow[b]{2}{*}{$\begin{array}{l}\text { Dog } \\
\text { No. }\end{array}$} & \multirow[b]{2}{*}{$\begin{array}{l}\text { Dose Rate } \\
\text { (rad/day) }\end{array}$} & \multirow[b]{2}{*}{$\begin{array}{c}\text { Days } \\
\text { Irradiated }\end{array}$} & \multirow[b]{2}{*}{$\begin{array}{c}\text { Total } \\
\text { Accumulated } \\
\text { Dose (rad) } \\
\end{array}$} & \multicolumn{3}{|c|}{ Terminal Hemogram (Days Before Death) } & \multicolumn{2}{|c|}{$\begin{array}{c}\text { Organ Weights at } \\
\text { Necropsy }\end{array}$} \\
\hline & & & & & $\begin{array}{c}\text { Erythrocytes } \\
\times 10^{6} \\
/ \mathrm{mm}^{3} \\
\end{array}$ & $\begin{array}{c}\text { Leukocytes } / \mathrm{mm}^{\prime} \\
\text { Corrected for } \\
\text { Nucleated RBC } \\
\end{array}$ & $\begin{array}{c}\text { Thrombocytes/ } \\
\mathrm{mm}^{\mathbf{3}}\end{array}$ & $\begin{array}{c}\text { Spleen }^{t} \\
(\mathrm{~g})\end{array}$ & $\begin{array}{c}\text { Liver } \\
(\mathrm{g}) \\
\end{array}$ \\
\hline $\mathrm{Ml}(9.3 \%)$ & $\begin{array}{l}1212 \\
1381\end{array}$ & $\begin{array}{r}3.75 \\
12.75\end{array}$ & $\begin{array}{l}1163 \\
1061\end{array}$ & $\begin{array}{r}4,361 \\
13,528\end{array}$ & $\begin{array}{l}1.83(0) \\
1.23(0)\end{array}$ & $\begin{array}{l}10,542 \\
44,463\end{array}$ & $\begin{array}{l}5,000 \\
2,000\end{array}$ & $\begin{array}{l}142.0 \\
218.2\end{array}$ & $\begin{array}{l}406.4 \\
477.8\end{array}$ \\
\hline M2 (37.0\%) & $\begin{array}{l}1472 \\
1678 \\
1913\end{array}$ & $\begin{array}{r}3.75 \\
7.5 \\
7.5\end{array}$ & $\begin{array}{r}1865 \\
405+(250)^{d} \\
405+(576)^{d}\end{array}$ & $\begin{array}{l}6,994 \\
3,037 \\
3,037\end{array}$ & $\begin{array}{l}0.89(0) \\
1.37(1) \\
1.15(3)\end{array}$ & $\begin{array}{l}39,015 \\
12,560 \\
64,630\end{array}$ & $\begin{array}{r}1,000 \\
1,000 \\
12,000\end{array}$ & $\begin{array}{r}82.5 \\
77.2 \\
139.6\end{array}$ & $\begin{array}{l}344.5 \\
416.1 \\
891.9\end{array}$ \\
\hline M4 (16.7\%) & $\begin{array}{l}1394 \\
2405\end{array}$ & $\begin{array}{r}12.75 \\
7.5\end{array}$ & $\begin{array}{r}1015 \\
200+(1841)^{d}\end{array}$ & $\begin{array}{r}12,941 \\
1,500\end{array}$ & $\begin{array}{l}4.49(1) \\
0.75(0)\end{array}$ & $\begin{array}{r}41,300 \\
5,381\end{array}$ & $\begin{array}{l}9,000 \\
3,000\end{array}$ & $\begin{array}{r}42.4 \\
310.0\end{array}$ & $\begin{array}{l}407.9 \\
650.0\end{array}$ \\
\hline M5a (5.6\%) & $\begin{array}{l}1382 \\
3241\end{array}$ & $\begin{array}{r}7.5 \\
1.875\end{array}$ & $\begin{array}{r}669 \\
1010\end{array}$ & $\begin{array}{l}5,017 \\
1,894\end{array}$ & $\begin{array}{l}1.81(1) \\
1.50(1)\end{array}$ & $\begin{array}{r}7,900 \\
39,608\end{array}$ & $\begin{array}{l}1,000 \\
3,000\end{array}$ & $\begin{array}{l}49.6 \\
94.0\end{array}$ & $\begin{array}{l}275.4 \\
458.1\end{array}$ \\
\hline MSb (1.9\%) & 2331 & 12.75 & $117+(1241)^{d}$ & 1,492 & $1.30(0)$ & 93,627 & 225,000 & 49.0 & 405.0 \\
\hline M6 (14.6\%) & $\begin{array}{l}1366 \\
1469 \\
2968\end{array}$ & $\begin{array}{r}3.75 \\
3.75 \\
1.875\end{array}$ & $\begin{array}{l}1949 \\
1440 \\
1831\end{array}$ & $\begin{array}{l}7,309 \\
5,400 \\
3,433\end{array}$ & $\begin{array}{l}1.21(0) \\
2.07(2) \\
1.23(1)\end{array}$ & $\begin{array}{r}34,148 \\
9,677 \\
51,264\end{array}$ & $\begin{array}{r}1,000 \\
2,000 \\
15,000\end{array}$ & $\begin{array}{l}188.8 \\
221.7 \\
200.0\end{array}$ & $\begin{array}{l}538.0 \\
526.8 \\
499.8\end{array}$ \\
\hline $\operatorname{EM}(7.4 \%)$ & $\begin{array}{l}1439 \\
2976\end{array}$ & $\begin{array}{r}3.75 \\
1.875\end{array}$ & $\begin{array}{l}1430 \\
1261\end{array}$ & $\begin{array}{l}5,362 \\
2,364\end{array}$ & $\begin{array}{l}1.73(1) \\
0.87(0)\end{array}$ & $\begin{array}{r}18,252 \\
8,839\end{array}$ & $\begin{array}{l}1,000 \\
5,000\end{array}$ & $\begin{array}{r}195.2 \\
90.5\end{array}$ & $\begin{array}{l}449.3 \\
548.1\end{array}$ \\
\hline M7a (1.9\%) & 4124 & 7.5 & $688^{\circ}$ & 5,160 & $2.19(1)$ & 800 & 22,000 & 41.0 & 236.8 \\
\hline$M 7 b(5.6 \%)$ & $\begin{array}{l}3255 \\
3871 \\
3907\end{array}$ & $\begin{array}{r}1.875 \\
7.5 \\
7.5\end{array}$ & $\begin{array}{r}841 \\
894 \\
1335\end{array}$ & $\begin{array}{r}1,577 \\
6,705 \\
10,012\end{array}$ & $\begin{array}{l}1.70(0) \\
2.53(5) \\
1.51(0)\end{array}$ & $\begin{array}{r}45,288 \\
5,300 \\
11,584\end{array}$ & $\begin{array}{r}90,000 \\
34,000 \\
2,075,000\end{array}$ & $\begin{array}{r}215.0 \\
40.6 \\
52.1\end{array}$ & $\begin{array}{l}489.6 \\
468.0 \\
447.1\end{array}$ \\
\hline Myelofibrosis & 3084 & 7.5 & 505 & 3,787 & $0.66(0)$ & 1,400 & 25,000 & 26.5 & 292.0 \\
\hline $\begin{array}{l}\text { ancidence of ob } \\
\text { - Exposures wer } \\
\text { - The mean weig } \\
{ }^{4} \text { Dogs received } \\
\text { - In-utero irradia } \\
\text { I Total observed } \\
\text { Note: No case o }\end{array}$ & $\begin{array}{l}\text { n } 22 \text { ho } \\
\text { SD, for } \\
\text { nated e } \\
\text { rom bir } \\
\text { ukemi }\end{array}$ & $\begin{array}{l}\text { on a total of } 5 \\
\text { y, } 7 \text { days/wee } \\
\text { ult nonexperin } \\
\text { es; }+ \text { (days) is } \\
\text { us } 63 \text { days. } \\
=6 \text {. } \\
\text { has been obse }\end{array}$ & $\begin{array}{l}\text { of radiation-ind } \\
{ }^{\circ 0} \mathrm{Co} \text { gamma sor } \\
\text { eagles: Spleen = } \\
\text { of days survivin } \\
292 \text { decensed, ac }\end{array}$ & $\begin{array}{l}\text { myeloid leukemi } \\
\text { conumencing at } \\
\pm 1.2 \text {, Liver }=3 \\
\text { lowing terminat } \\
\text { nonirradiated, } \mathrm{cc}\end{array}$ & $\begin{array}{l}B / A N L=\text { French } \\
\text { imately } 400 \text { day } \\
\text { 13.5. } \\
\text { irradiation. } \\
\text { dogs. }\end{array}$ & $\begin{array}{l}\text { rican-Brit } \\
\text { ge. }\end{array}$ & tional Laborat & & . \\
\hline
\end{tabular}


Of the cases discussed here, the terminal hemogram in the majority shows severe anemia and thrombocytopenia. Total leukocyte and differential counts vary from an aleukemic-subleukemic count to a leukemic blast hiatus. Necropsy findings in the majority of cases show moderate to marked hepatosplenomegaly, lymphadenopathy, and pulpy hypercellular marrow. The degree of leukemic cell infiltration in both hematopoietic and nonhematopoietic tissues varies widely. A well-defined preleukemic syndrome is observed in most dogs at periods from 1 month to $2 \mathrm{y}$ prior to death and has been described: Fritz, Tolle, and Seed 1985; Seed et al. 1978; Seed, Chubb, and Tolle 1981; Tolle et al. 1982a,b, 1983. A number of cases in the Argonne study have previously been reported: Fritz, Norris, and Tolle 1973; Fritz et al. 1982; Seed et al. 1977, 1982; Tolle, Fritz, and Norris 1977; Tolle et al. 1979a,b; Tolle, et al. 1979a,b; Tolle et al. 1982a,b.

\section{DESCRIPTIONS}

\section{Myeloid Leukemia Without Maturation (FAB-M1) (SNOVET M-98613)}

In the bone marrow, the predominant cell is the myeloblast, although there may be a minimal population of later cell stages (promyelocytes and myelocytes). The blast population exceeds $50 \%$ of the nonerythroid series, it is nongranular, and it contains one or more prominent nucleoli. A high percentage of blasts may be micromyeloblasts. A small proportion may stain with Sudan black or myeloperoxidase (MPO) (Figures 2.1-2.3).

\section{Myeloid Leukemia With Maturation (FAB-M2) (SNOVET M-98613)}

The majority of marrow cells are in the myeloblast-myelocyte stage, although later stages are not uncommon, and in some cases mature neutrophils may be present in large numbers (neutrophilic variant). However, these cells generally show abnormalities, i.e., pseudo-Pelger-Huët segmentation (Fritz, Tolle, and Seed 1985; Tolle et al., 1982b) and hypo- or agranularity (Figures 2.4-2.6).

\section{Hypergranular Promyelocytic Leukemia (FAB-M3)}

This condition has not been observed in the Argonne studies and is not described in this atlas.

\section{Myelomonocytic Leukemia (FAB-M4) (SNOVET M-98603)}

The marrow shows a predominance of cells of both granulocytic and monocytic differentiation, with the myeloblast-myelocyte stages constituting more than $50 \%$ of the nonerythroid series; the monoblast, promonocyte population makes up more than $20 \%$. The peripheral blood shows a high percentage of promonocytes and monocytes, with the monocytes possibly having band-like and segmented nuclei. The combined specific/nonspecific esterase stain is useful in diagnosis (Figures 2.7-2.9).

\section{Monocytic Leukemia Without Maturation (FAB-M5a) (SNOVET M-98913)}

The predominant marrow cell is the monoblast, with delicate chromatin, prominent nucleoli, and cytoplasmic buds or pseudopodia. The blast component usually exceeds $80 \%$ of the total cell population. A low percentage ( $10 \%$ to $15 \%$ ) of promonocytes may be present, as well as a minor granulocytic component, which is usually less than $10 \%$ (Figures $2.10-2.15$ ).

Monocytic Leukemia With Maturation (FAB-M5b) (SNOVET M-98913)

In the marrow a monoblast-promonocyte hiatus is the prominent feature, but a high percentage of mature monocytes may be found in the peripheral blood. Many of these mature monocytes show band-like and segmented nuclei (Figures 2.16-2.17). 


\section{Myeloid Leukemia}

\section{Erythroleukemia (FAB-M6) (SNOVET M-98403)}

The bone marrow is characterized by a mixed population of rubriblasts and myeloblasts, with the myeloblast-myelocyte population being less than $25 \%$ to $30 \%$ of the total nucleated cells. The erythroid series shows severe maturation arrest, asynchronous nuclear/cytoplasmic maturation, megaloblastic features, and giant forms. The later stages, i.e., prorubricytes, rubricytes, and metarubricytes, show bizarre morphologic features such as karyorrhexis, micronuclei, and double nucleated forms. Aberrant mitotic figures may be prominent. A reverse myeloid:erythroid ratio is always seen (Figures 2.18-2.22).

\section{Erythremic Myelosis (EM) (SNOVET M-98403)}

The marrow erythroid series shows the same features as in M6, but granulocyte maturation/differentiation is relatively normal, and there is little or no increase in myeloblasts. Micromegakaryocytes may be present, but cytoplasmic maturation appears to be morphologically normal. The myeloid:erythroid ratio varies widely, but is rarely greater than 1:1 (Figures 2.23-2.24).

\section{Megakaryocytic Leukemia Without Maturation (FAB-M7a) (SNOVET M-99103)}

The marrow shows a high percentage $(>80 \%)$ of megakaryoblasts and micromegakaryocytes. The blast cells vary in size from small round cells with little cytoplasm (which resemble lymphoblasts) to cells that are three to four times larger. The majority have a round nucleus, with fairly dense chromatin and one to four distinct nucleoli. Double-nucleated micromegakaryocytes are not uncommon. Many cells have a somewhat eccentric nucleus. A moderate degree of cytoplasmic vacuolization may be seen and may represent saccules of the developing demarcation membrane system. Mature but aberrant megakaryocytes are present in small numbers, as are mature naked megakaryocyte nuclei. Acetylcholinesterase (ACHE) stain is helpful in diagnosis on peripheral blood buffy coat and bone-marrow films, and ultrastructural platelet peroxidase is diagnostic in transmission electron microscopic sections (Figures 2.25-2.26).

\section{Megakaryocytic Lukemia With Maturation (FAB-M7b) (SNOVET M-99103)}

The predominant marrow cell is the recognizable but highly aberrant megakaryocyte and micromegakaryocyte. A small percentage of megakaryoblasts is also seen. Large cytoplasmic fragments, giant platelets, and aberrant vacuolated and hypogranular platelets are seen in the peripheral blood. In many cases, there is increased marrow fibrosis, which may result in inadequate material from aspirates for film preparation (Figures 2.27-2.31 and 2.34-2.35).

Myelofibrosis (SNOVET M-49000/T-06000)

Because myelofibrosis is included with the myeloproliferative disorders and is often seen in the myeloid leukemias, particularly the megakaryocytic and chronic myelogenous forms, we have included it in this atlas. Nonleukemic myelofibrosis is a prominent endpoint in our radiation studies, constituting $9.1 \%,(6 / 66$ cases of either myeloproliferative disease or myelodysplastic syndrome) (Figures 2.32-2.33). 


\section{REFERENCES}

Bennett, J. M., D. Catovsky, M. T. Daniel, G. Flandrin, D. A. G. Galton, H. R. Gralnick, and C. Sultan. 1976. Proposals for the classification of the acute leukemias. Br. J. Haematol. 33:451458.

Bennett, J. M., D. Catovsky, M. T. Daniel, G. Flandrin, D. A. G. Galton, H. R. Gralnick, and C. Sultan. 1985a. Criteria for the Diagnosis of Acute Leukemia of Megakaryocyte Lineage (M7), A Report of the French-American-British Cooperative Group. Ann. Int. Med. 103:460-462.

Bennett, J. M., D. Catovsky, M. T. Daniel, G. Flandrin, D. A. G. Galton, H. R. Gralnick, and C. Sultan. 1985b. Proposed Revised Criteria for the Classification of Acute Myeloid Leukemia, A Report of the French-American-British Cooperative Group. Ann. Int. Med. 103:460-462.

Fritz, T. E., W. P. Norris, and D. V. Tolle. 1973. Myelogenous leukemia and related myeloproliferative disorders in beagles continuously exposed to ${ }^{60} \mathrm{Co}$ gamma-radiation. Bibl. Haematol. 39:170-188.

Fritz, T. E., D. V. Tolle, D. E. Doyle, T. M. Seed, and S. M. Cullen. 1982. Hematologic Responses of Beagles Exposed Continuously to Low Doses of 60-Co Gamma-Radiation, pp. 229240. In: Experimental Hematology Today, Part VIII. S. J. Baum, G. D. Ledney, and S. Thierfelder, Eds. S. Karger, New York.

Fritz, T. E., D. V. Tolle, and T. M. Seed. 1985. The Preleukemic Syndrome in Radiation-Induced Myelogenous Leukemia and Related Myeloproliferative Disorders, pp. 87-100. In: The Preleukemic Syndrome (Hematopoietic Dysplasia, G. C. Bagby; Jr., Ed. CRC Press, Boca Raton, FL.

Seed, T. M., D. V. Tolle, T. E. Fritz, R. L. Devine, C. M. Poole, and W. P. Norris. 1977. Irradiation-induced erythroleukemia and myelogenous leukemia in the beagle dog: Hematology and ultrastructure. Blood 50:1061-1079.

Seed, T. M., D. V. Tolle, T. E. Fritz, S. M. Cullen, L. V. Kaspar, and C. M. Poole. 1978. HemoPathological Consequences of Protracted Gamma Irradiation in the Beagle: Preclinical Phases of Leukemia Induction, Late Biological Effects of Ionizing Radiation, pp. 531 -545. Vol. I, IAEA International Symposium, LAEA-SM-224/308, International Atomic Energy Agency, Vienna.

Seed, T. M., G. T. Chubb, and D. V. Tolle. 1981. Sequential Changes in Bone Marrow Architecture during Continuous Low Dose Gamma Iradiation, pp. 16, 61-72. In: Scanning Electron Microscopy/1981/IV. O. Johari, R. M. Albrecht, and T. M. Seed, Eds. SEM, Inc., AMF O'Hare, Chicago, IL.

Seed, T. M., G. T. Chubb, D. V. Tolle, T. E. Fritz, C. M. Poole, D. E. Doyle, L. S. Lombard, and L. V. Kaspar. 1982. The Ultrastructure of Radiation-Induced Endosteal Myelofibrosis in the Dog, pp. 377-391. In: Scanning Electron Microscopy/ 1982/I. O. Johari, S. A. Bhatt, and I. C. Pontarelli, Eds. SEM, Inc., AMF O'Hare, Chicago, IL.

SNOVET. 1984. Systemized Nomenclature of Medicine, Microglossary for Veterinary Medicine. J. L. Palotay and D. J. Rothwell, Eds. American Veterinary Medical Association, Schaumburg, II.

Tolle, D., T. E. Fritz, and W. P. Norris. 1977. Radiation-induced erythroleukemia in the beagle dog. A hematologic summary of five cases. Am. J. Pathol. 87:499-510.

Tolle, D., T. M. Seed, T. E. Fritz, L. S. Lombard, C. M. Poole, and W. P. Norris. 1979a. Acute monocytic leukemia in an irradiated beagle. Vet. Pathol. 16:243-254. 


\section{Myeloid Leukemia}

Tolle, D., T. M. Seed, T. E. Fritz, and W. P. Norris, W. P. 1979b. Irradiation-Induced Canine Leukemia: A Proposed New Model. Incidence and Hematopathology, pp. 247-256. In:

Experimental Hematology Today, 1979, S. J. Baum and G. D. Ledney, Eds. Springer-Verlag, New York.

Tolle, D. V., T. M. Seed, S. M. Cullen, C. M. Poole, T. E. and Fritz. 1982a. Aberrant Megakaryocytopoiesis Preceding Radiation-Induced Leukemia in the Dog, pp. 367-376. In: Scanning Electron Microscopy/1982/I. 0. Johari, R. M. Albrecht, and T. M. Seed, Eds. SEM, Inc., AMF O'Hare, Chicago, IL.

Tolle, D. V., T. E. Fritz, T. M. Seed, S. M. Cullen, L. S. Lombard, and C. M. Poole. 1982b. Leukemia Induction in Beagles Exposed Continuously to ${ }^{60} \mathrm{Co}$ Gamma-Irradiation, pp. 241-249. In: Hematopathology, Experimental Hematology Today, Part VIII, S. J. Baum, G. D. Ledney, and S. Theirfelder, Eds. S. Karger, New York.

Tolle, D. V., S. M. Cullen, T. M. Seed, and T. E. Fritz. 1983. Circulating micromegakaryocytes preceding leukemia in three dogs exposed to $2.5 \mathrm{R} /$ day gamma radiation. Vet. Pathol. 20:111114. 

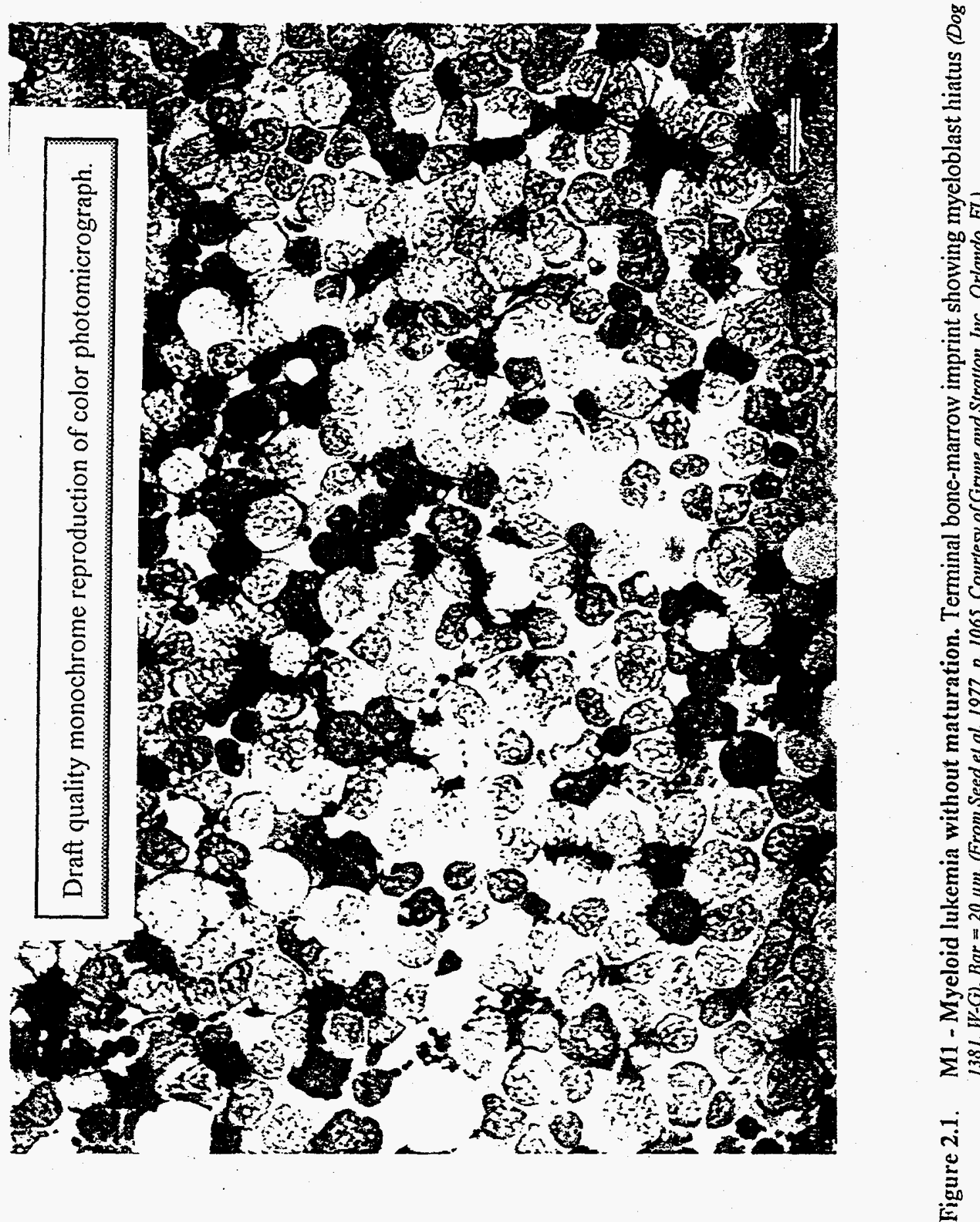


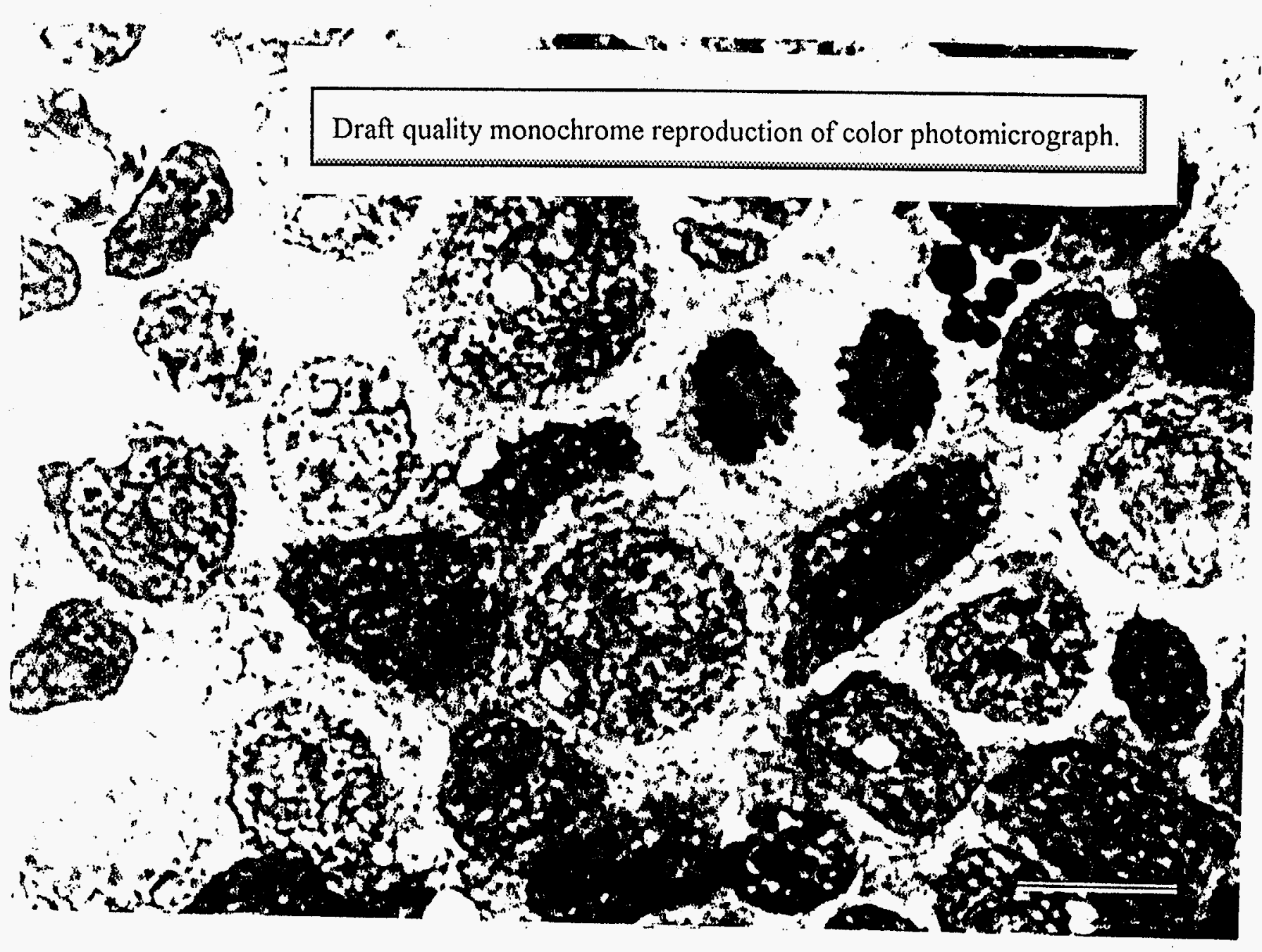

Figure 2.2. M1 - Myeloid leukemia without maturation. Same preparation as Figure 2.1 at higher magnification. $B a r=10 \mu m$. 


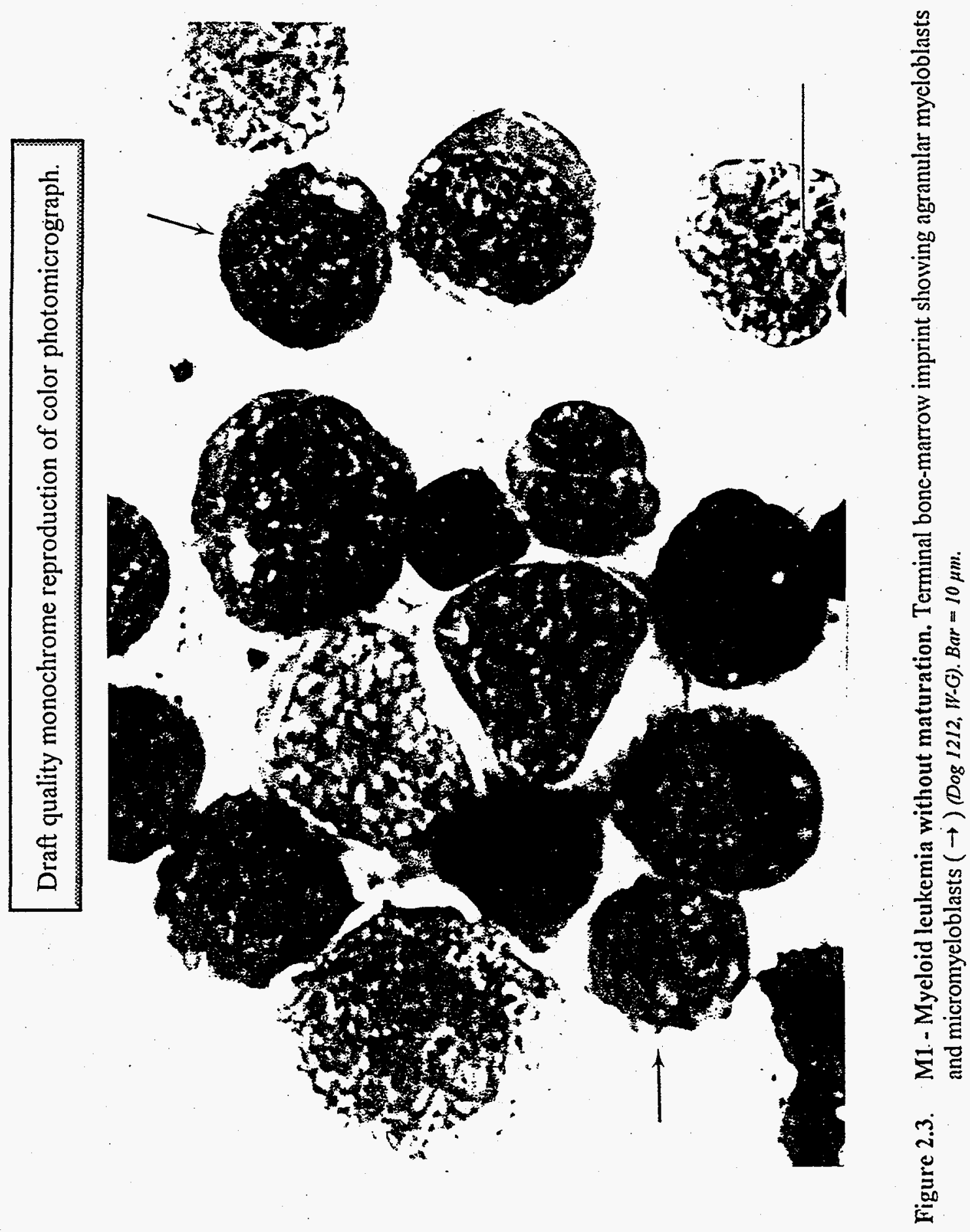




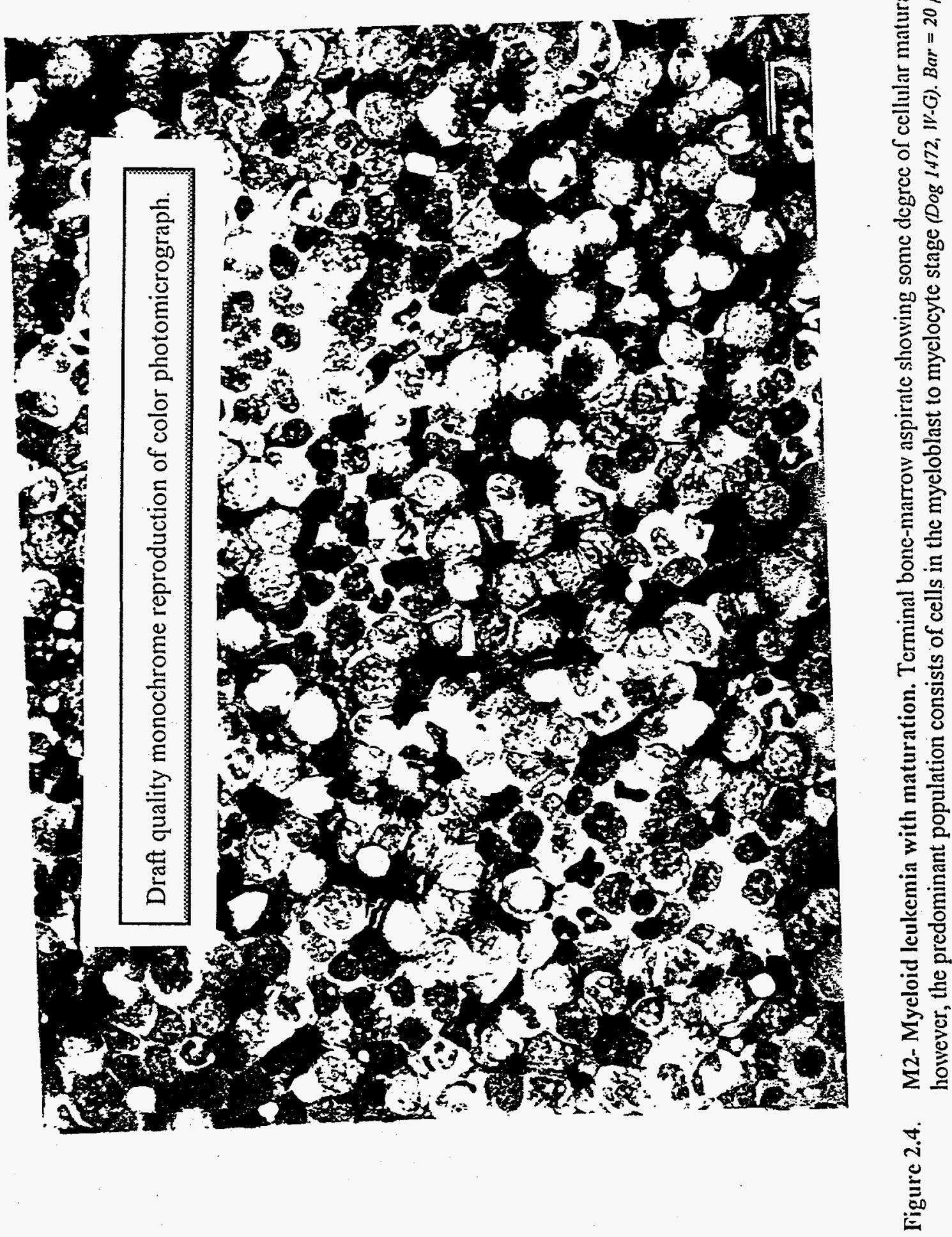




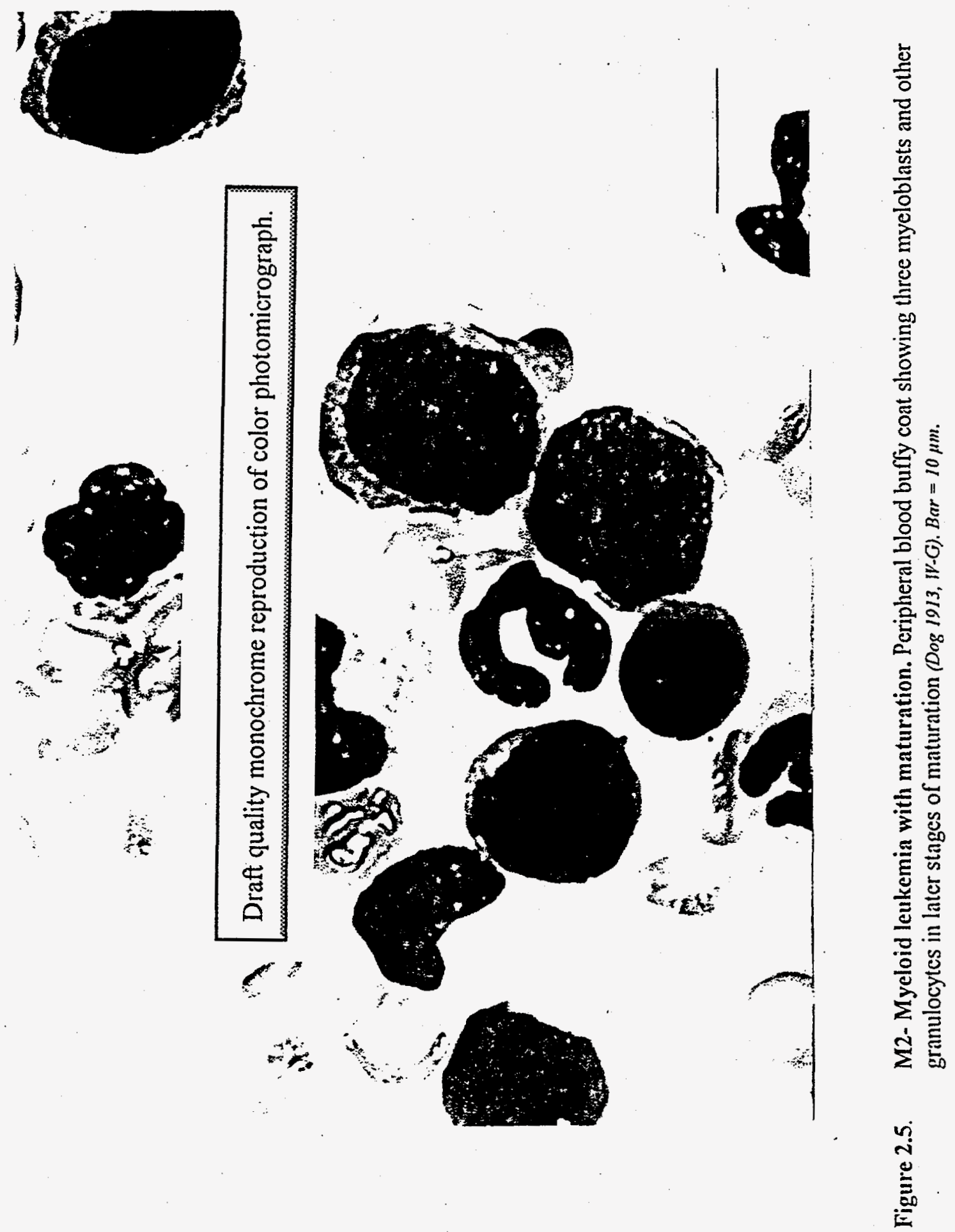




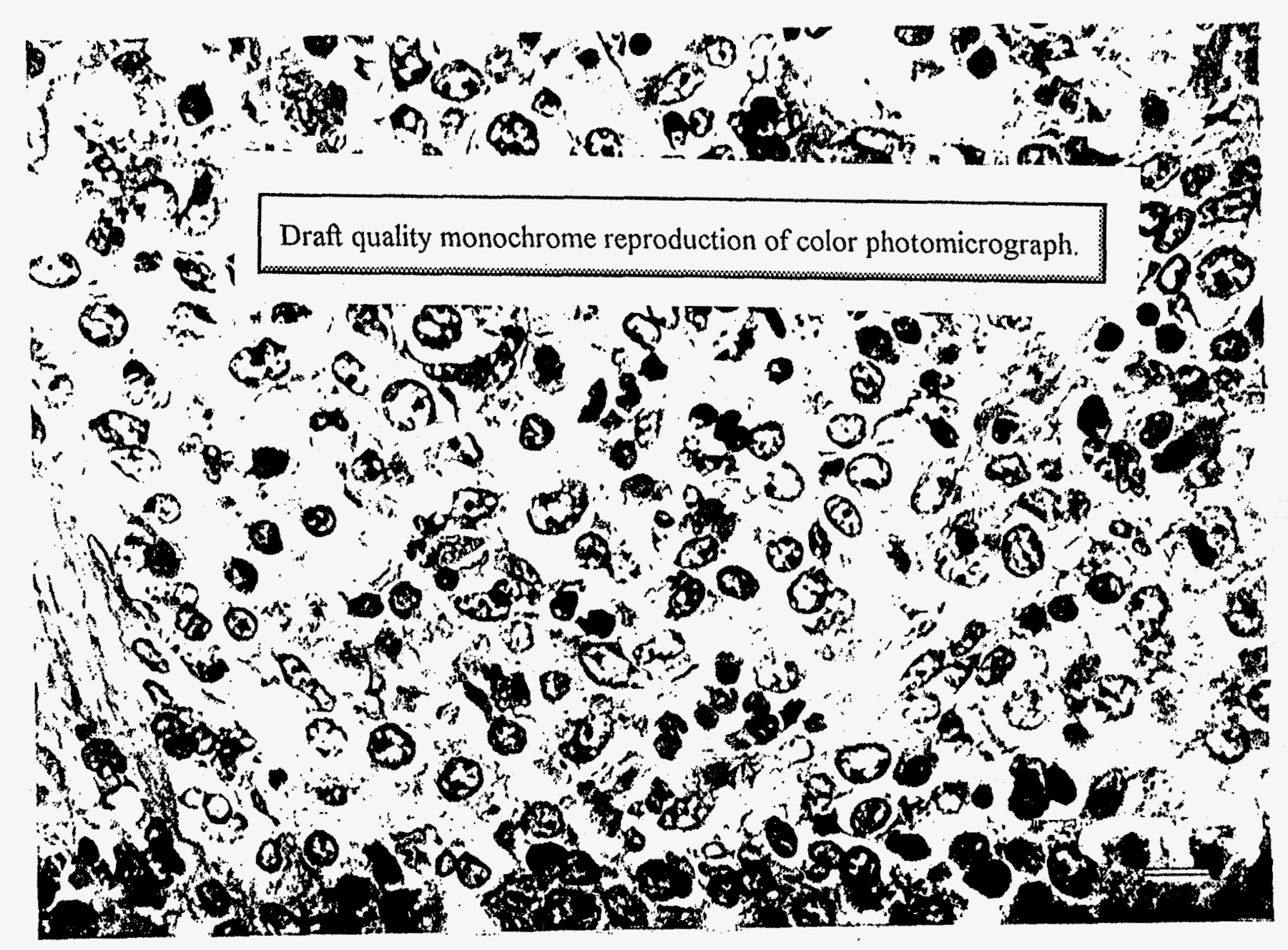

Figure 2.6. M2- Myeloid leukemia with maturation. Spleen section showing marked lcukcmic cell infiliration (Dog J678, H\&\&5). $B a r=10 \mu m$. 


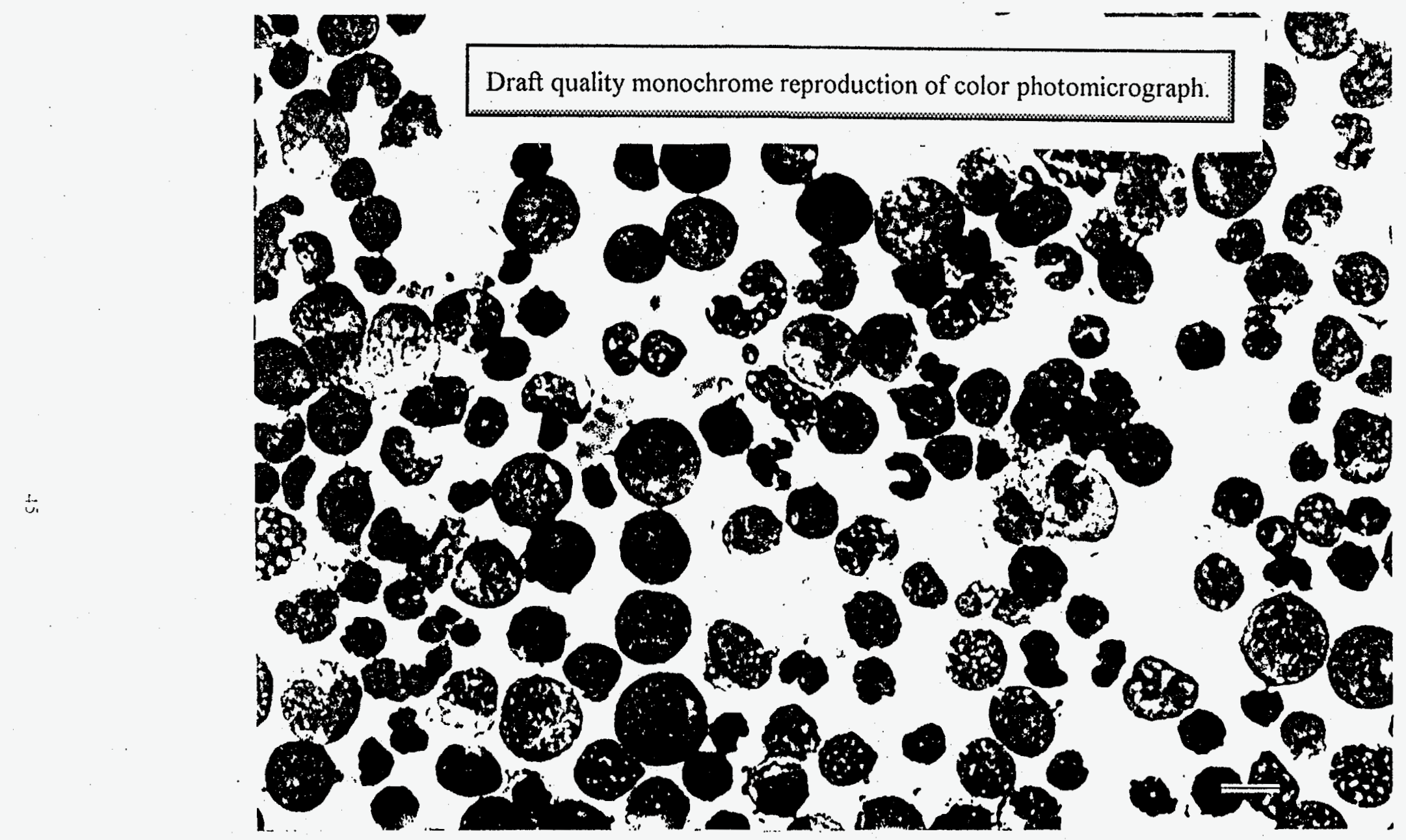

Figure 2.7. M4 - Myelomonocytic leukemia. Terminal bone-marrow imprint showing the predominant population to be a mixture of myeloblasts and micromyeloblasts as well as monoblasts and promonocytes. There are a few granulocytic cells in later maturation stages and occasional mature monocytes present (Dog 1394, W-G). Bar = $10 \mu \mathrm{m}$. 


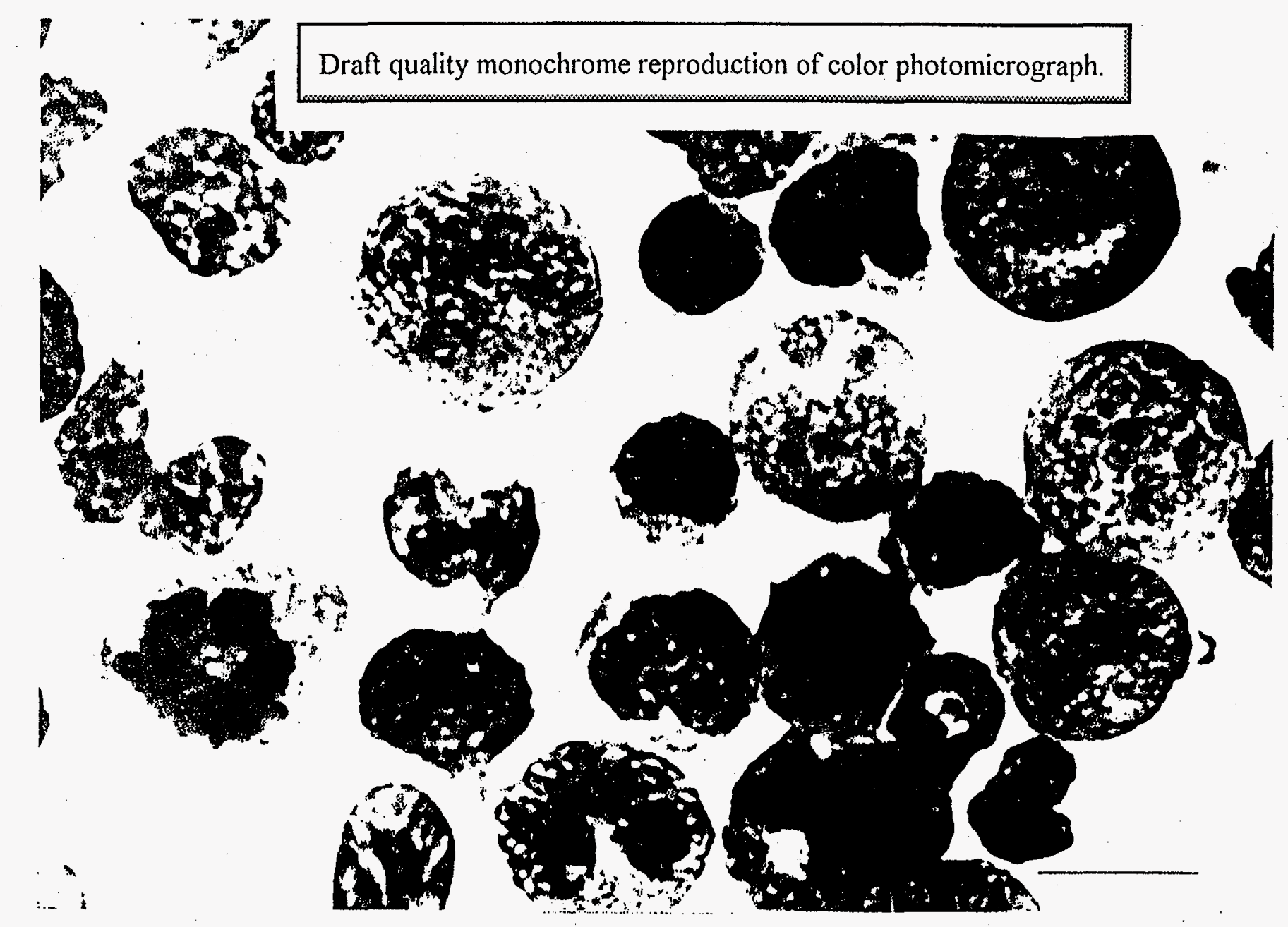

Figure 2.8. M4 - Myelomonocytic leukemia. Same preparation as Figure 2.7, but at a higher magnification. Bar $=10 \mu m$. 


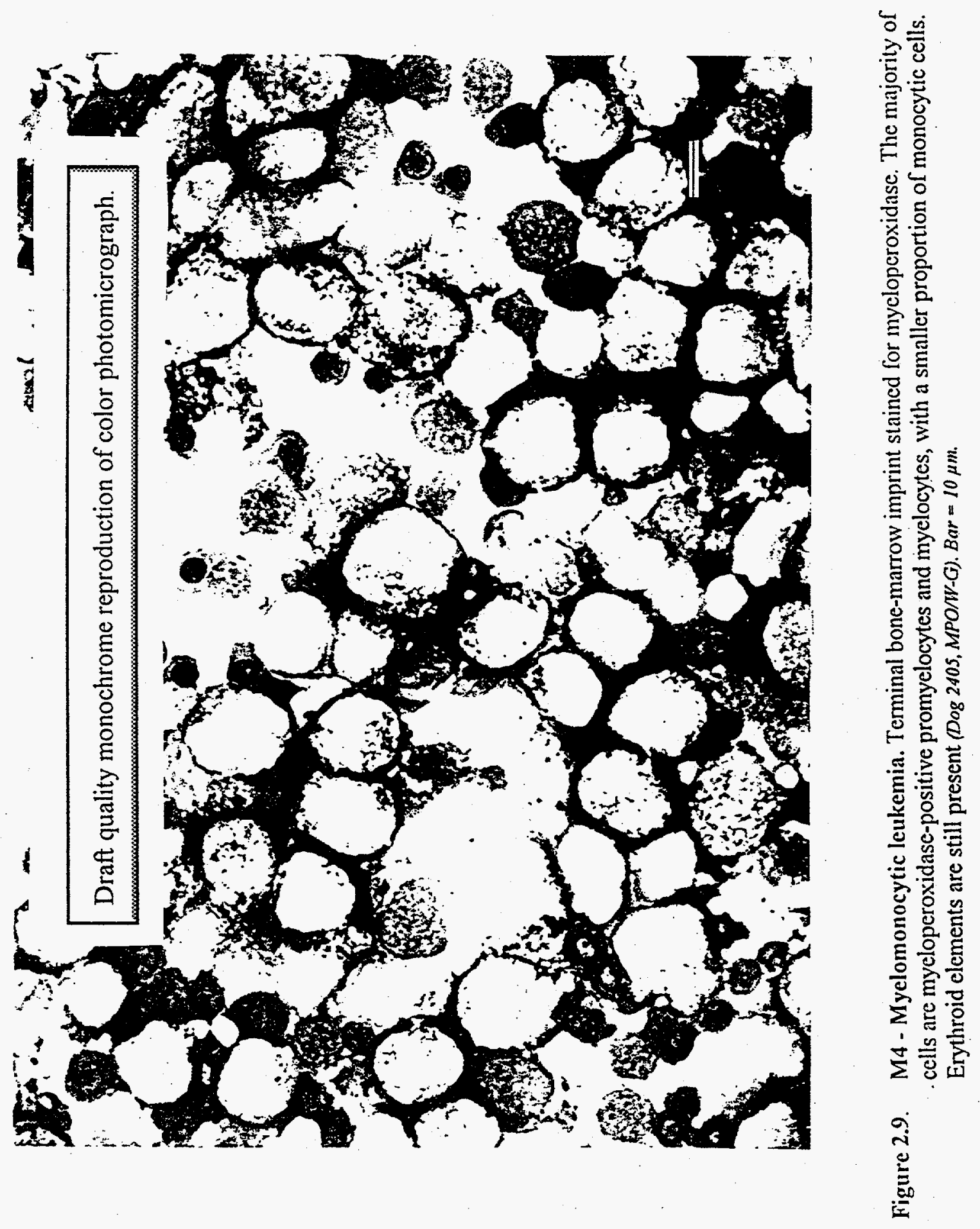



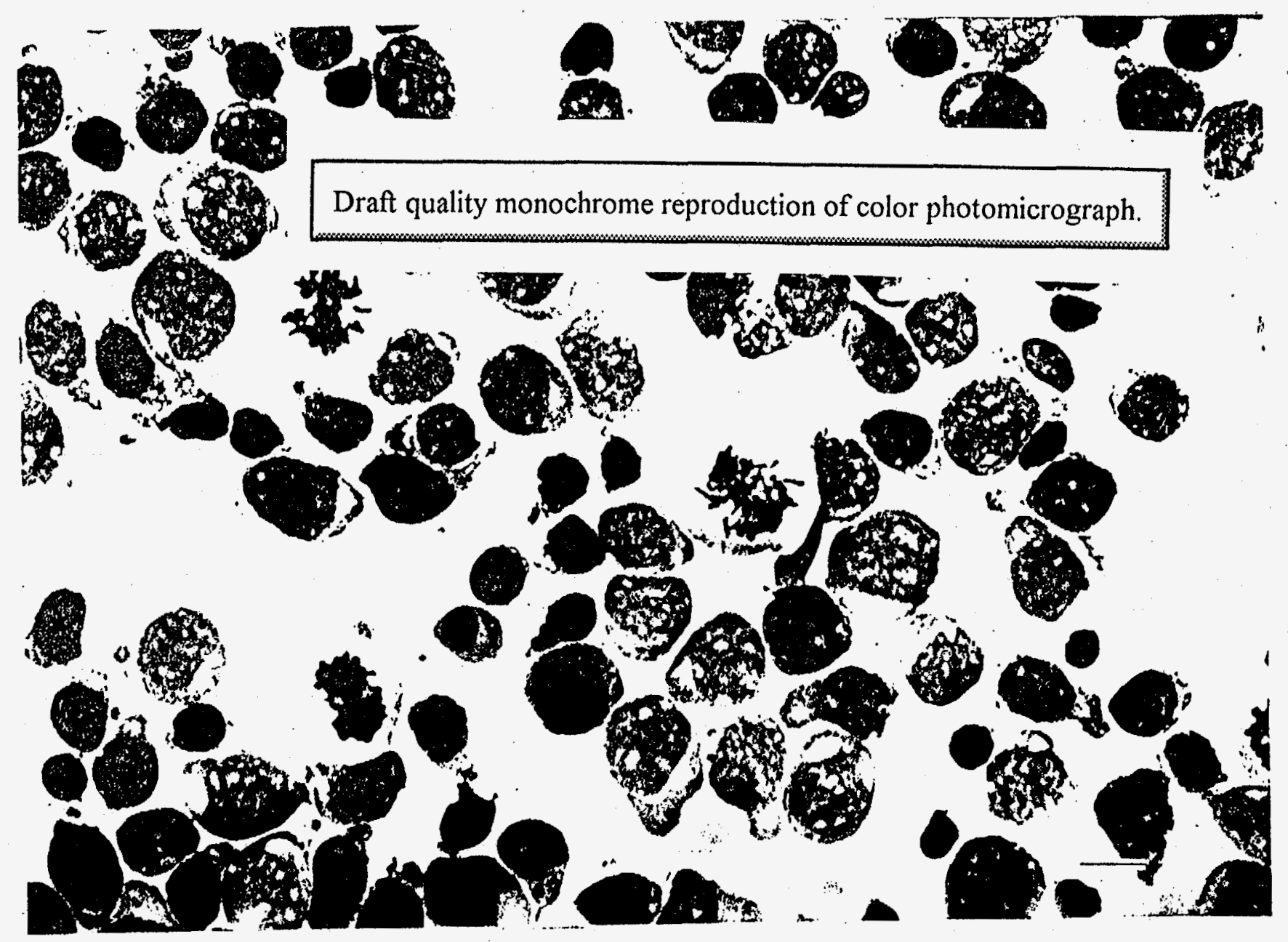

Figure 2.10. M5a-Monocytic leukemia without maturation. Bonc-marrow aspirate 6 days before death, showing a monoblast hiatus. Note the prominent nucleoli, delicate chromatin, and cytoplasmic buds or psecudopodia (Dog 324I, W-G). Bar $=10 \mu \mathrm{m}$. (From Tolle, et al., 1982b, p. 245. Courtesy S. Karger AG, Basel). 


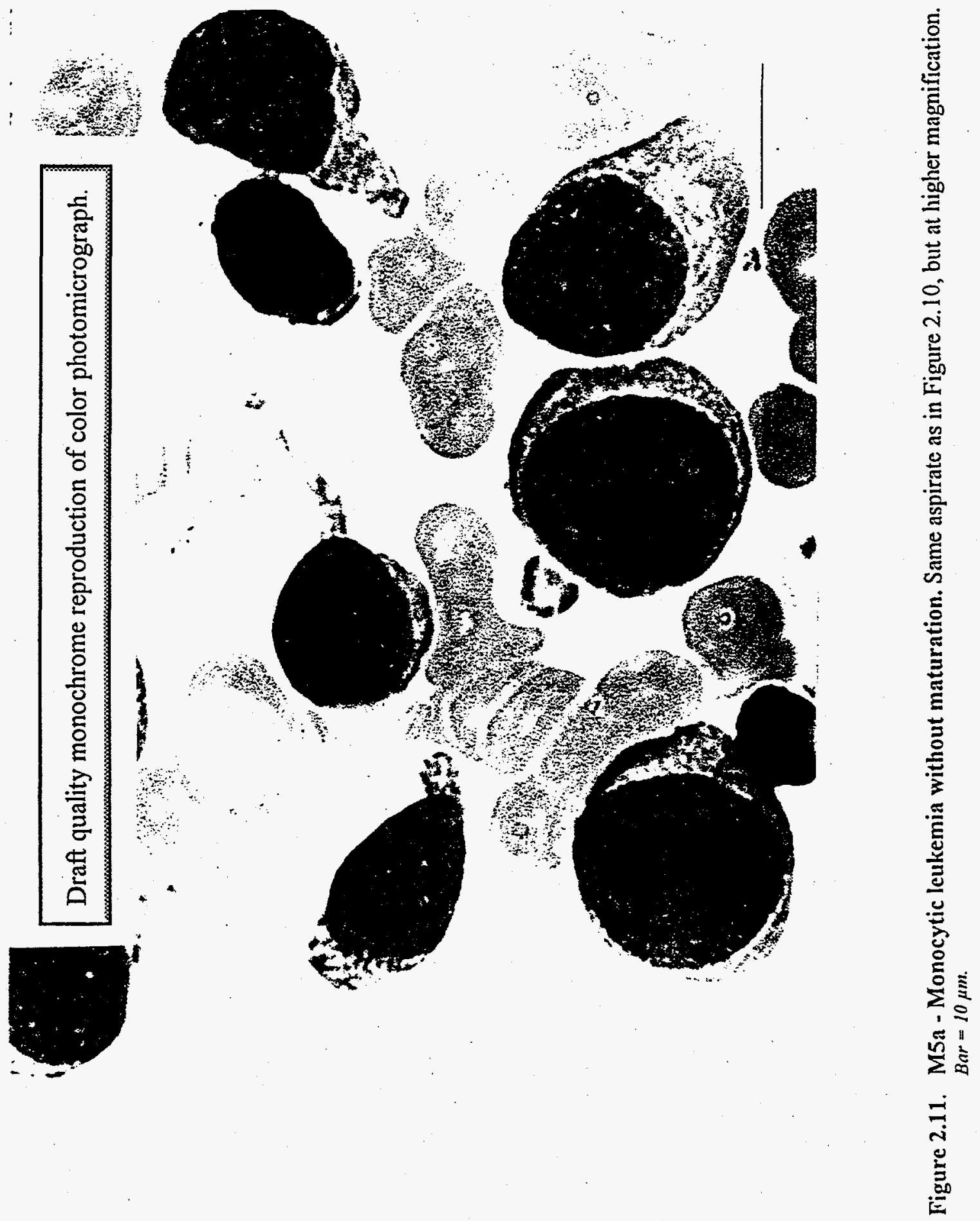




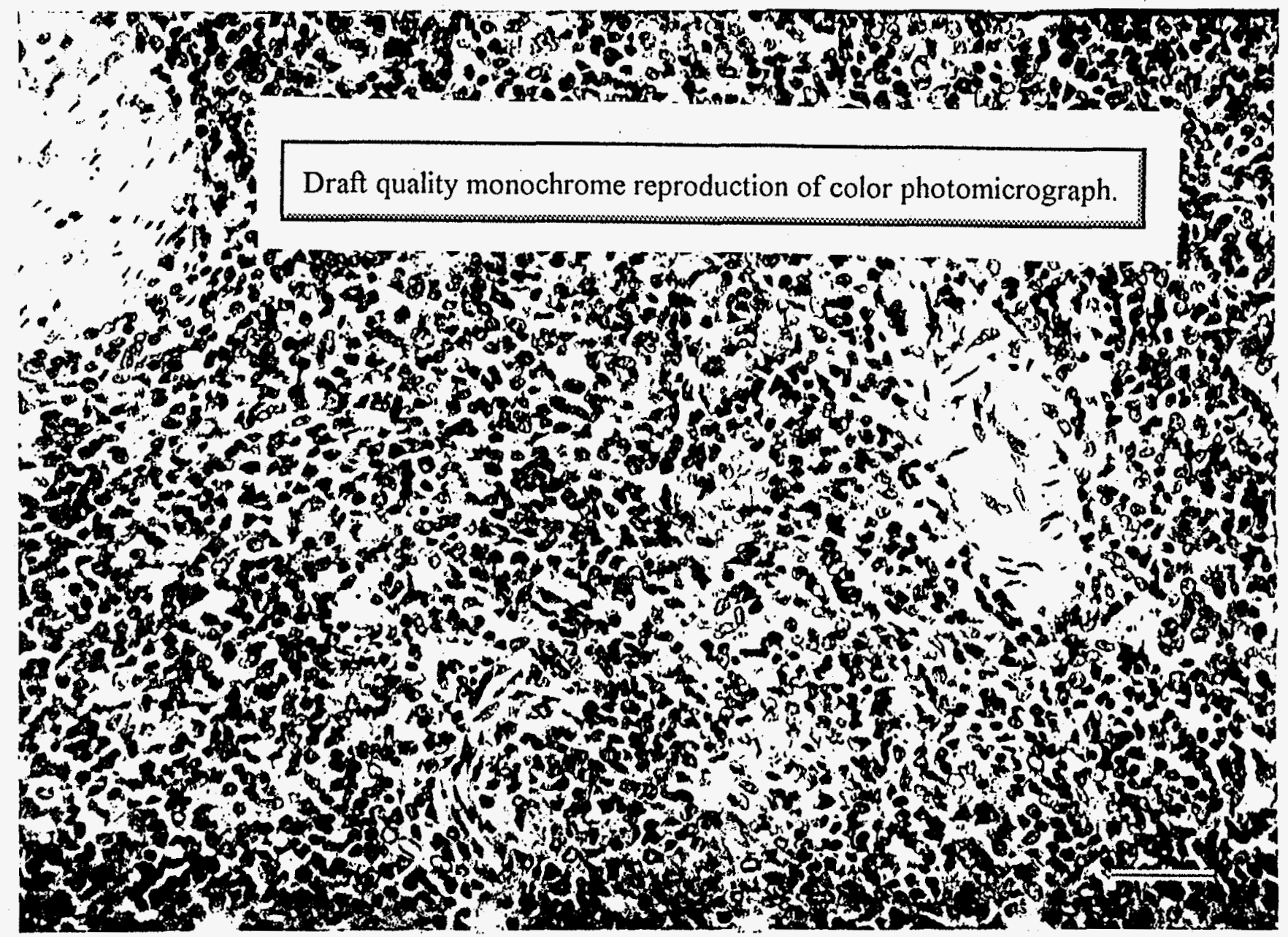

Figure 2.12. M5a - Monocytic leukemia without maturation. Spleen section from case shown in Figure 2.10 showing complete replacement of normal tissue with leukemic cells (H\&EC). Bar $=40 \mu \mathrm{m}$. 


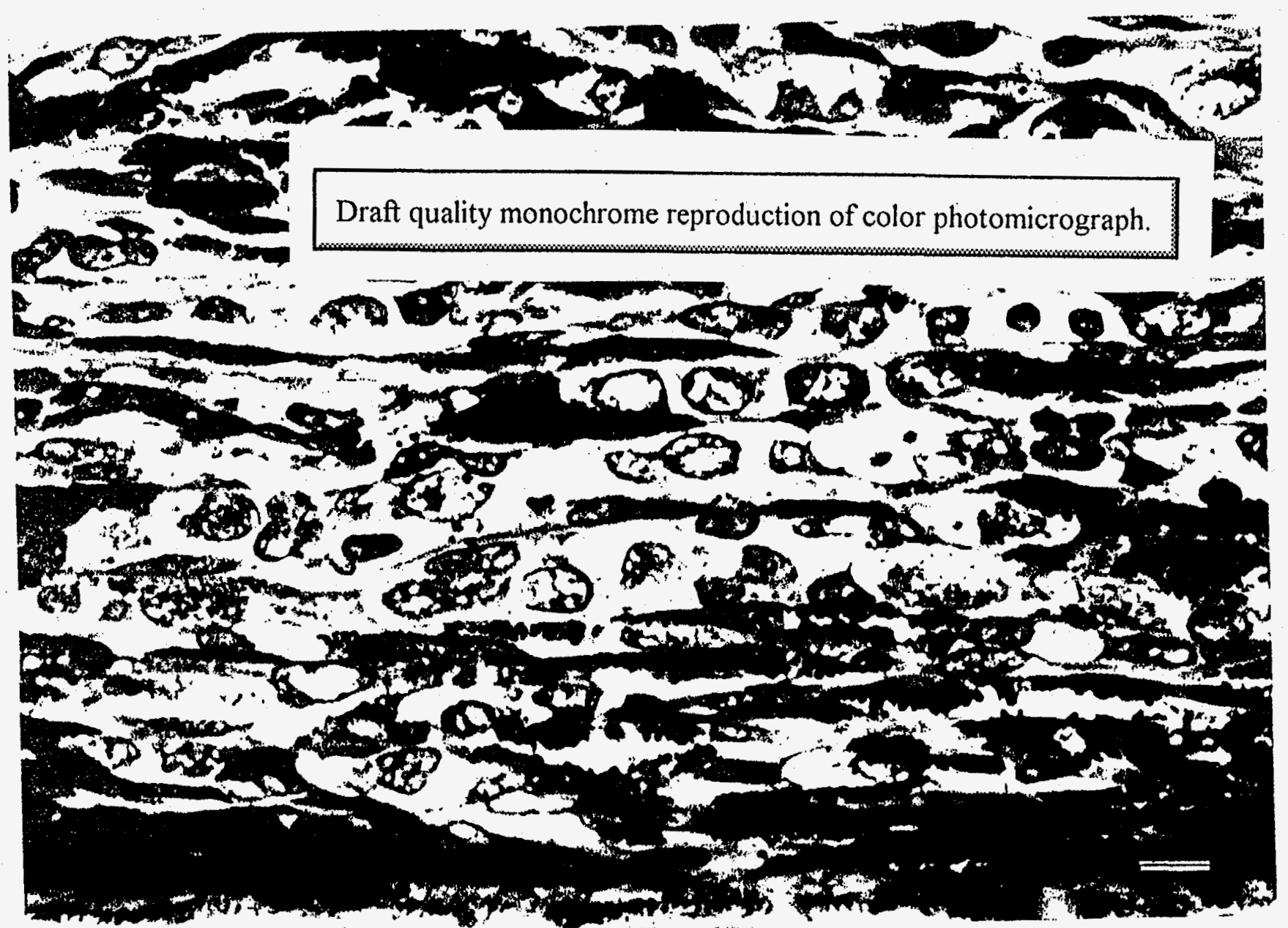

Figure 2.13. M5a - Monocytic leukemia without maturation. Eye section from case shown in Figure 2.10: note leukemic infiltration of the choroid of the tunica media (H\&E). Bar $=10 \mu \mathrm{m}$. (From Tolle et al. 19\$2b, p. 246. Courlesy S. Karger AG. Basel). 


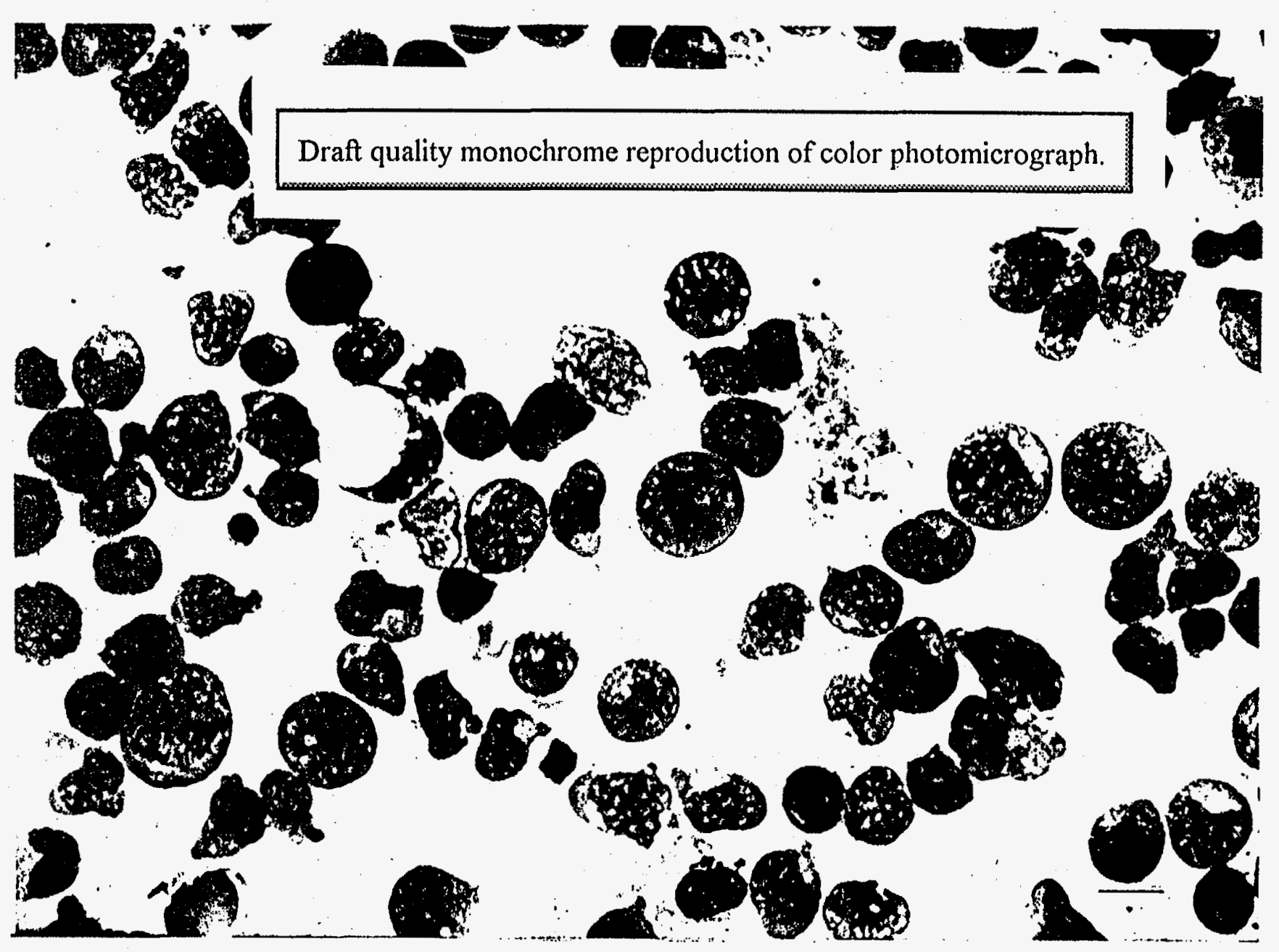

Figure 2.14. M5a-Monocytic leukemia without maturation. Terminal bone-marrow aspirate showing a predominance of monoblasts with more condensed chromatin than seen in the blast cells shown in Figures 2.10 and 2.11. Nuclear folding is also more cvident in this case (Dog 1382, $\mathrm{W}-\mathrm{G}$ ). Bar $=10 \mu \mathrm{m}$. 


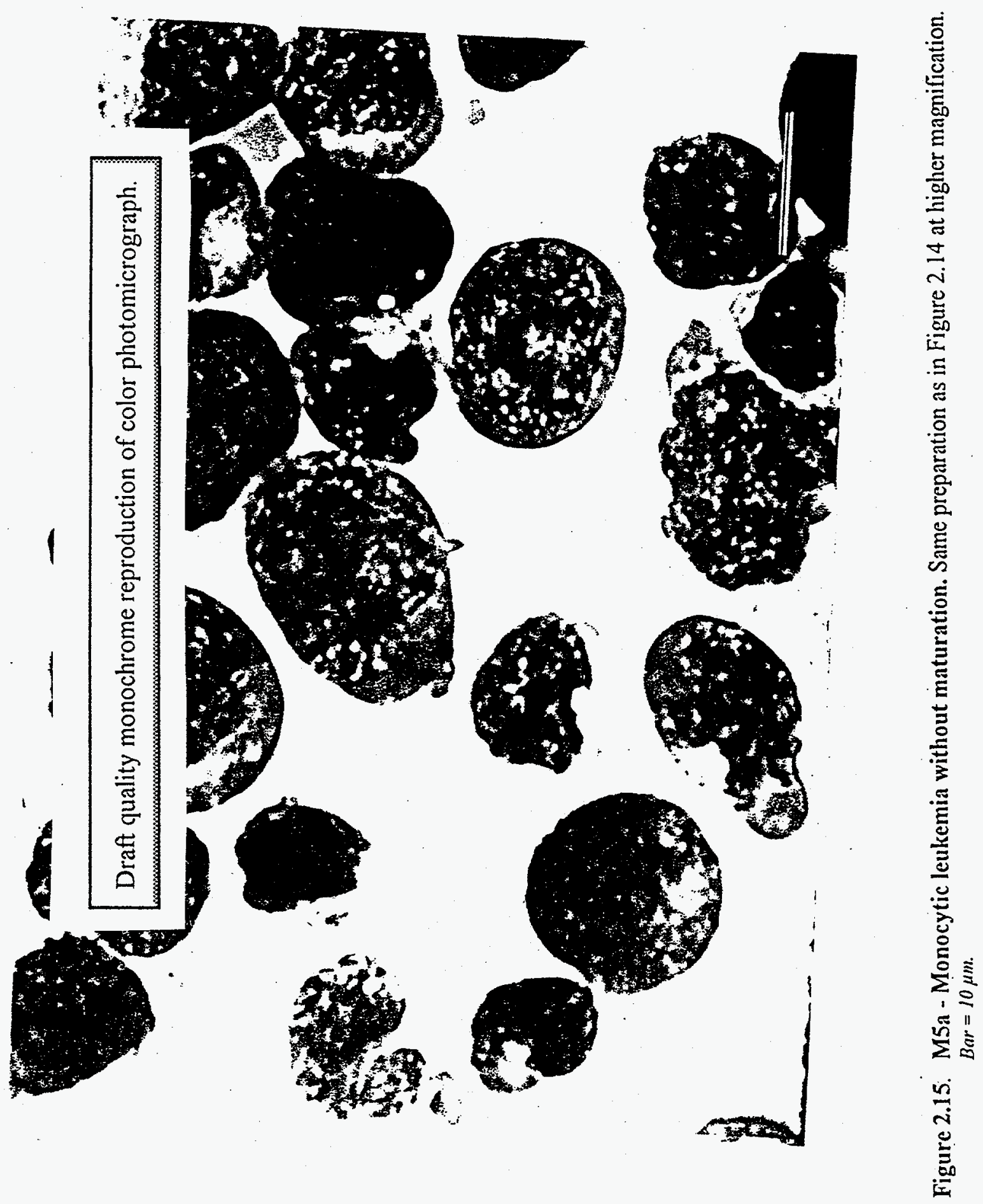




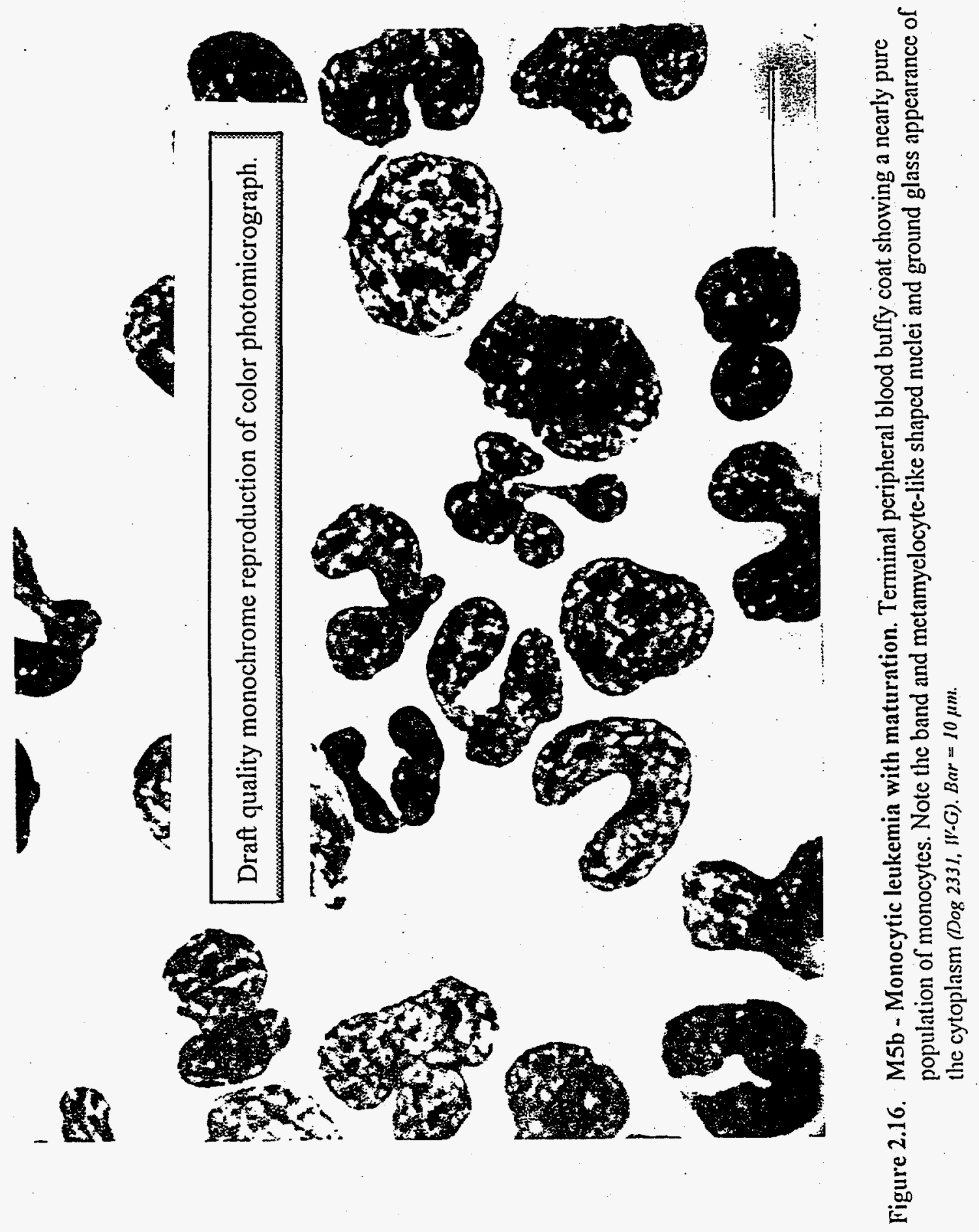




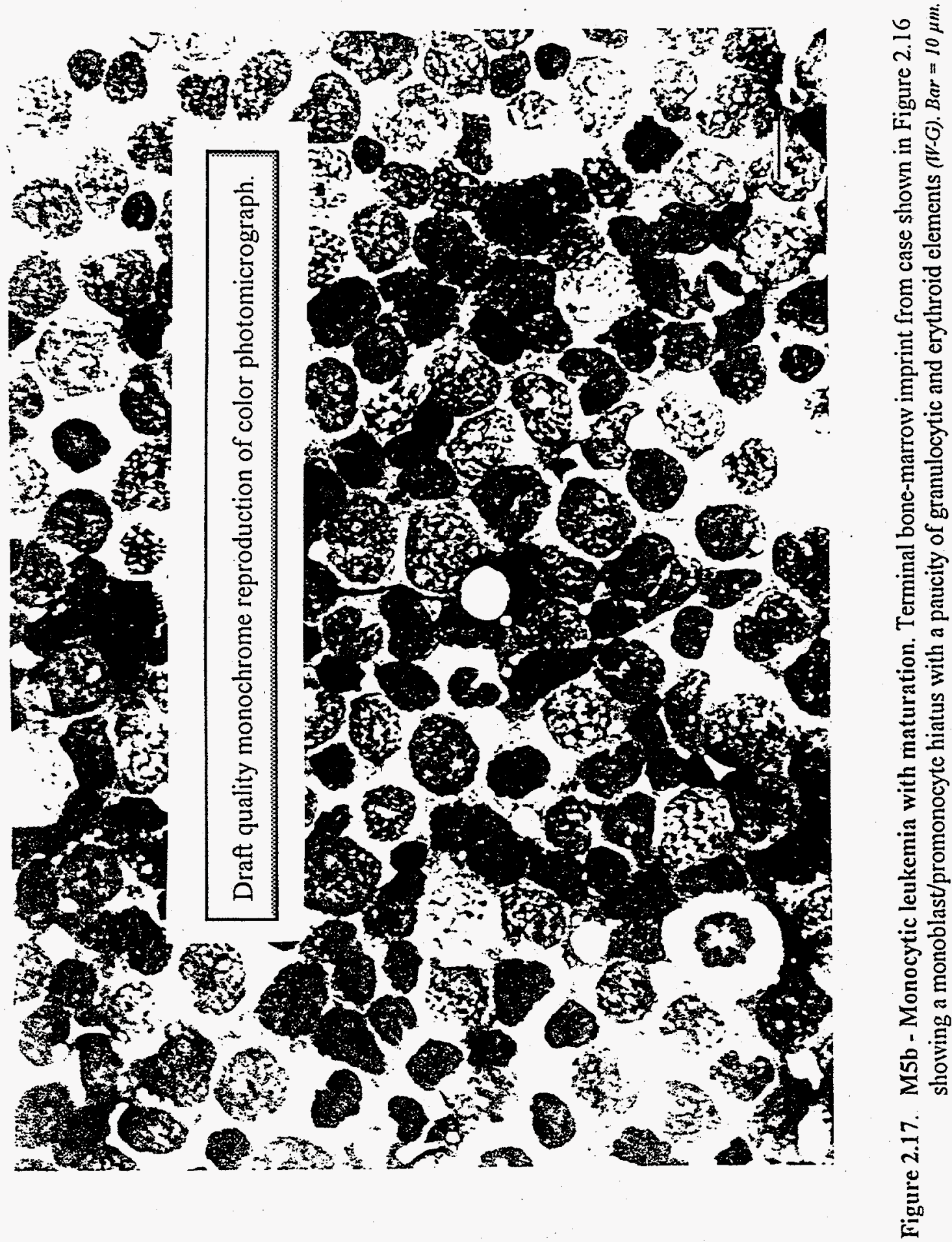




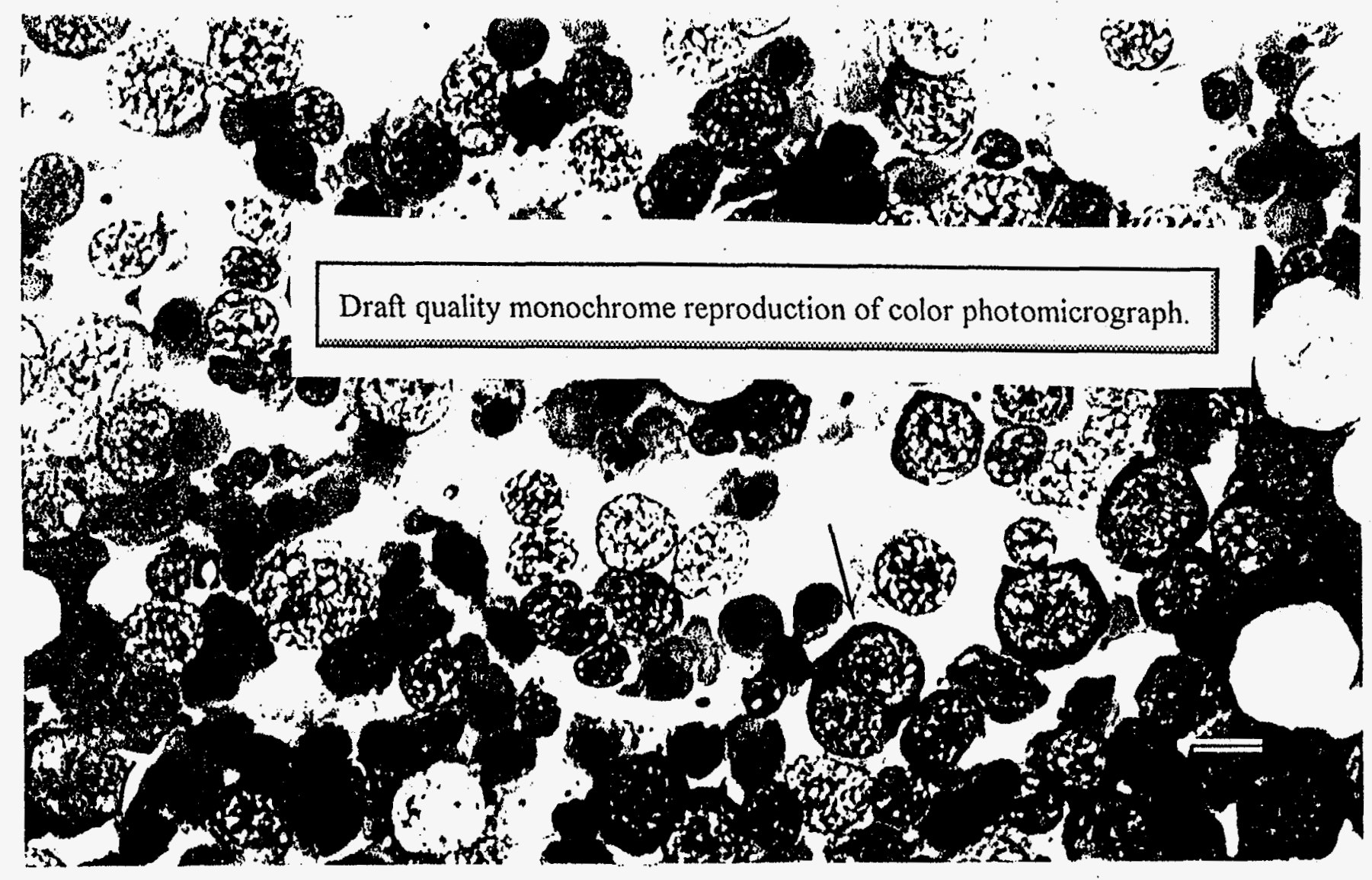

Figure 2.18. M6 - Erythroleukemia. Periodic acid Schiff (PAS) stain on terminal bone-marrow imprint showing marked crythroblastic hyperplasia, with a large proportion of cells being rubriblasts. There is a smaller proportion of myeloblasts and myelocytes. Note occasional double-nucleated rubriblasts $(\rightarrow)$, megaloblastic features, and degree of karyorrhexis in the later erythroblast stages. The erythroblasts are PAS-negative. (Dog 1366, PAS/W-G) $B a r=10 \mu m$. 


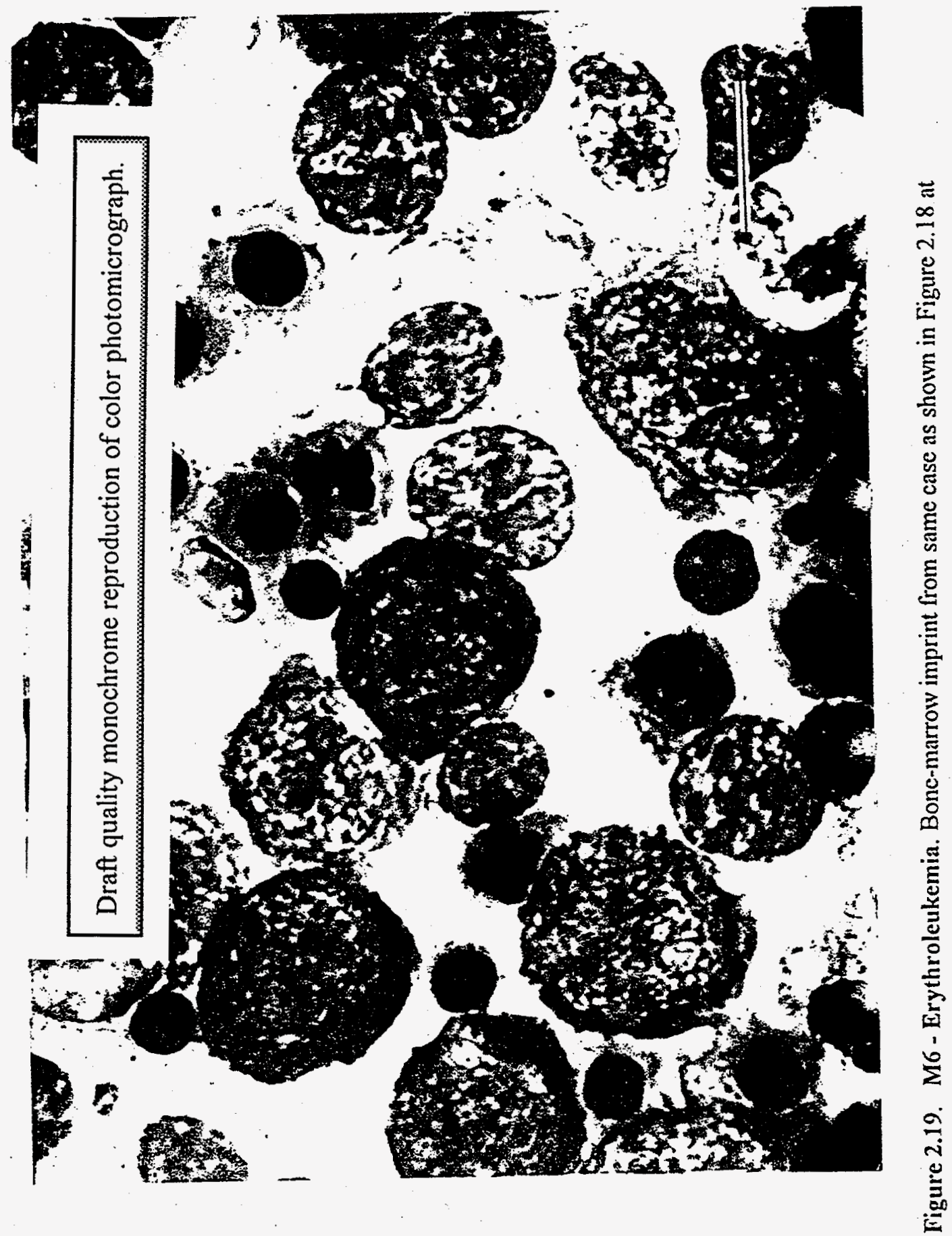




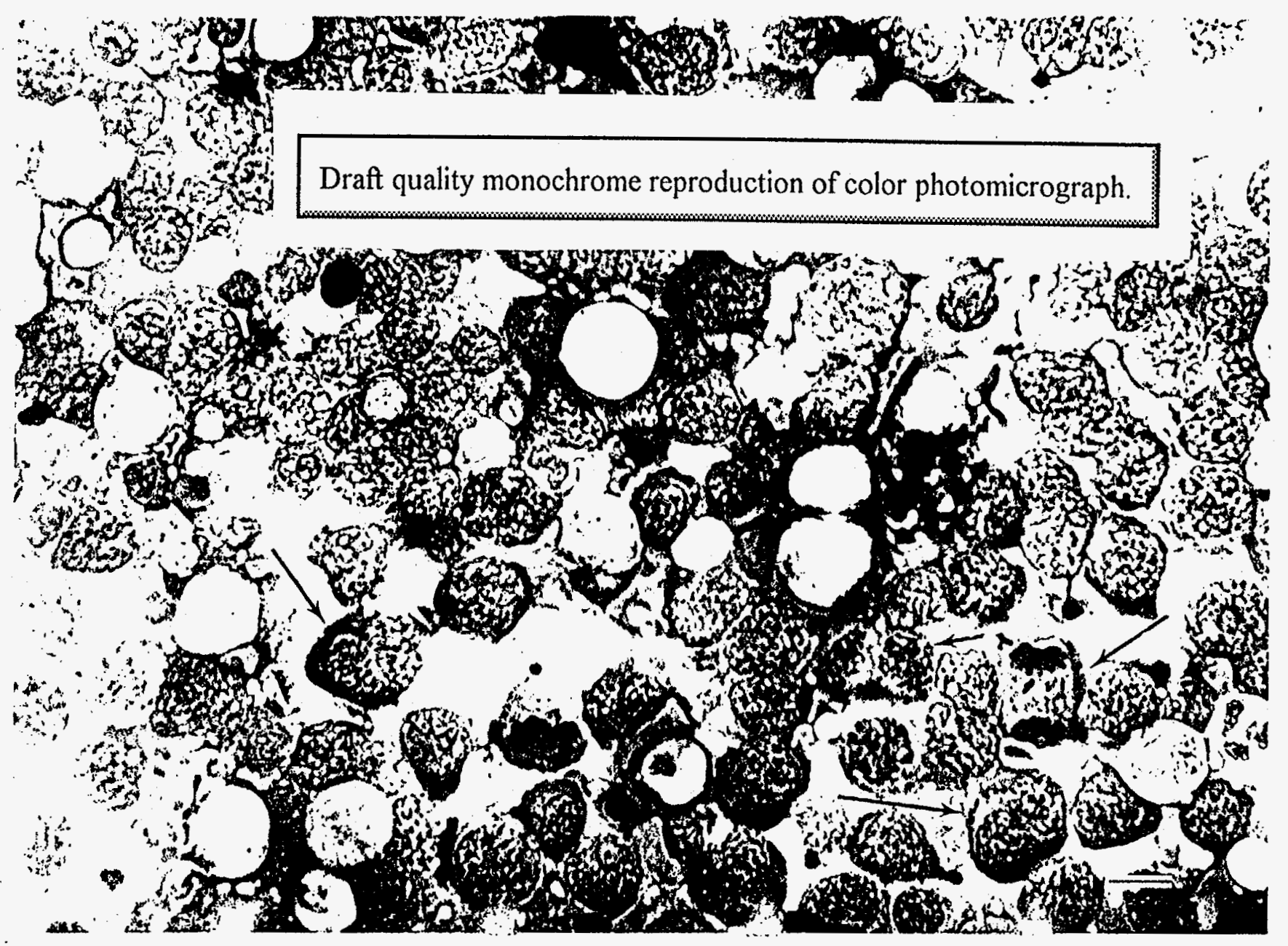

Figure 2.20. M6 - Erythroleukemia. Spleen imprint showing a rubriblast hiatus. Many cells are stripped of cytoplasm and appear as naked nuclei. Note occasional double-nucleated forms, mitotic figures (-1), and few granulocytes. (Dog 1469, W-G). Bar $=10 \mu \mathrm{m}$. (From Tolle, Fritz, and Norris 1977, p. 509. Courlesy of Harper and Row Publishers, Inc., Philadelphia, PA). 


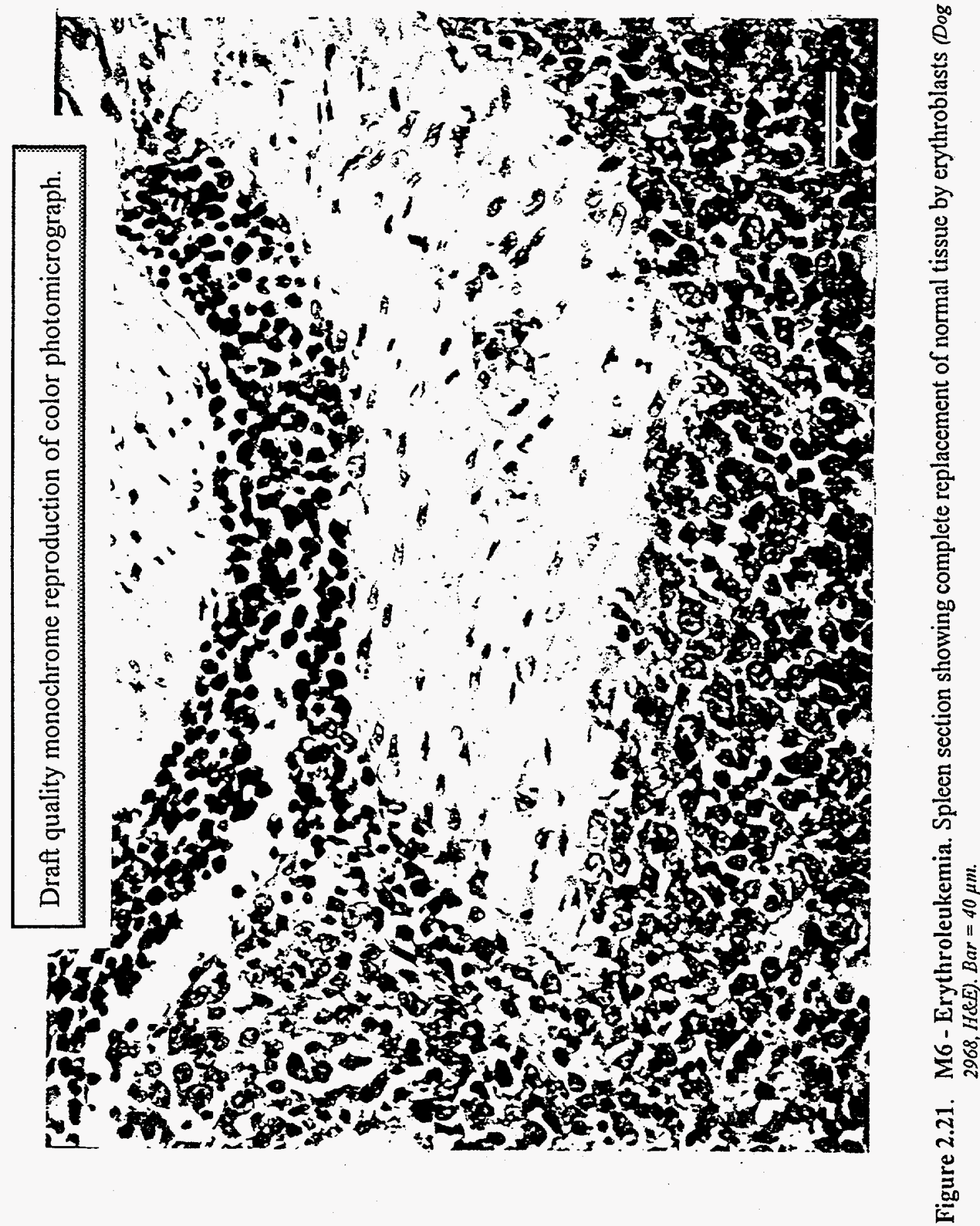




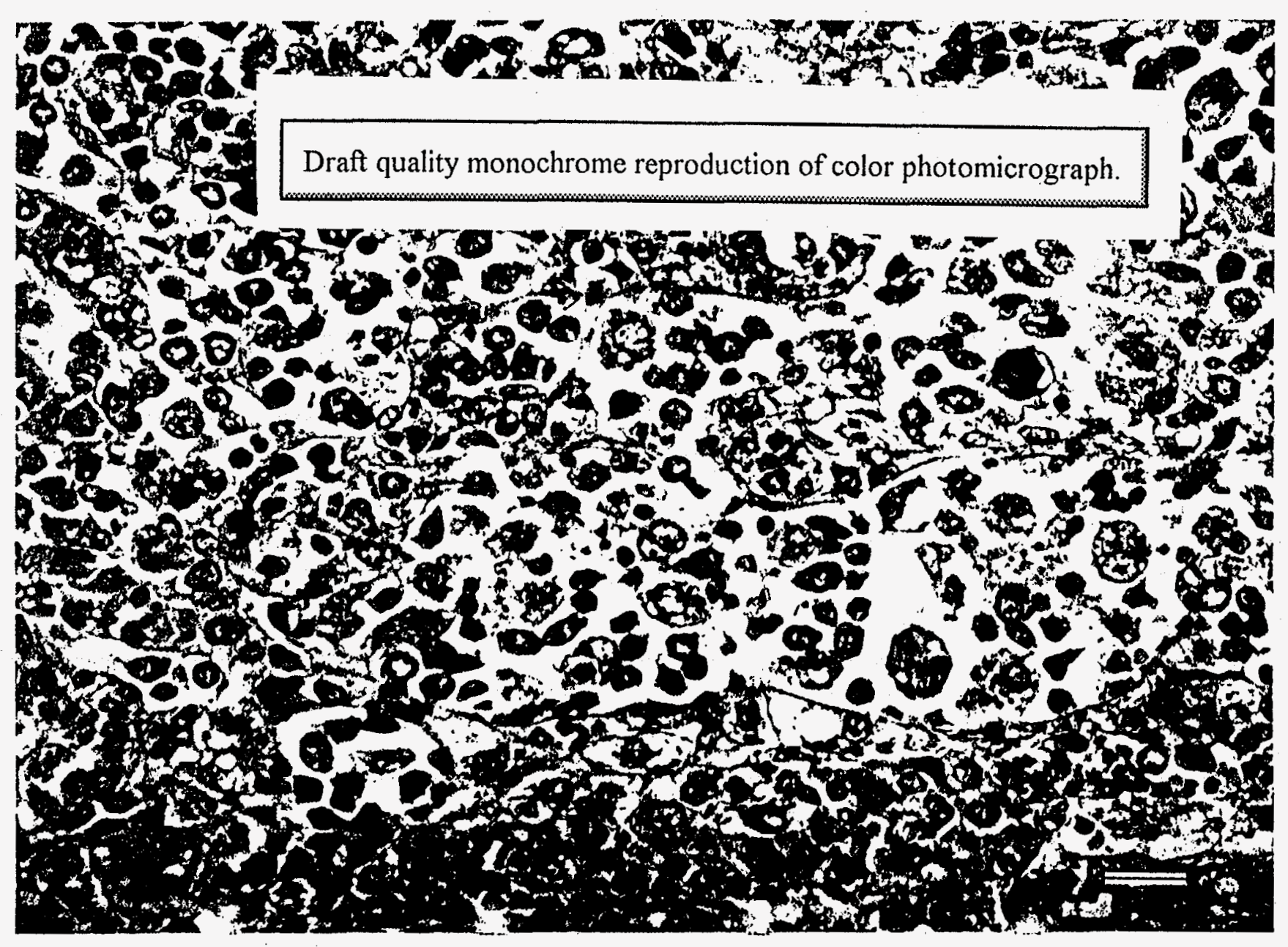

Figure 2.22. M6 - Erythroleukemia. Liver section from case shown in Figure 2.21, showing infiltration by erythroblasts (HHE). Bar $=20 \mu m$. 


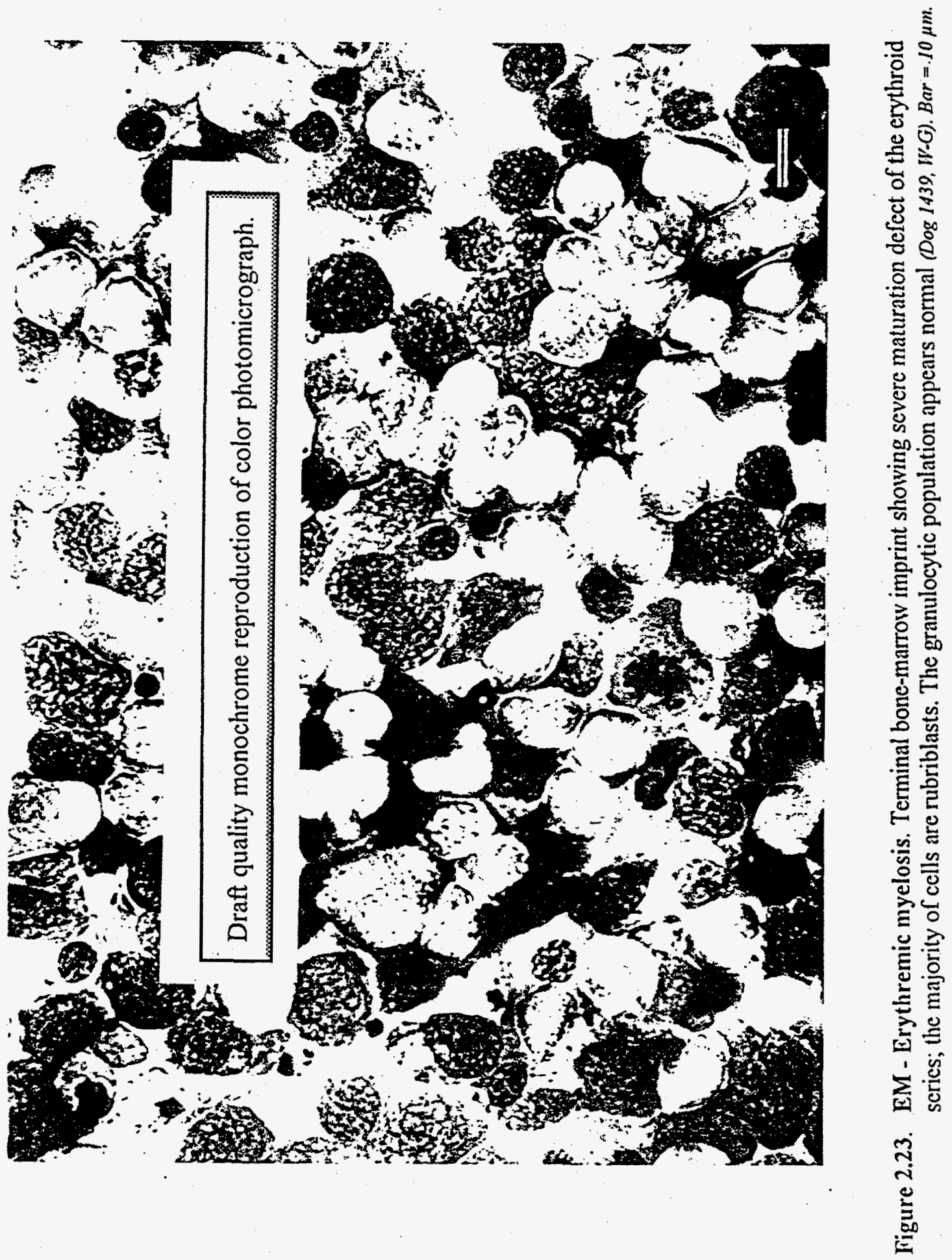




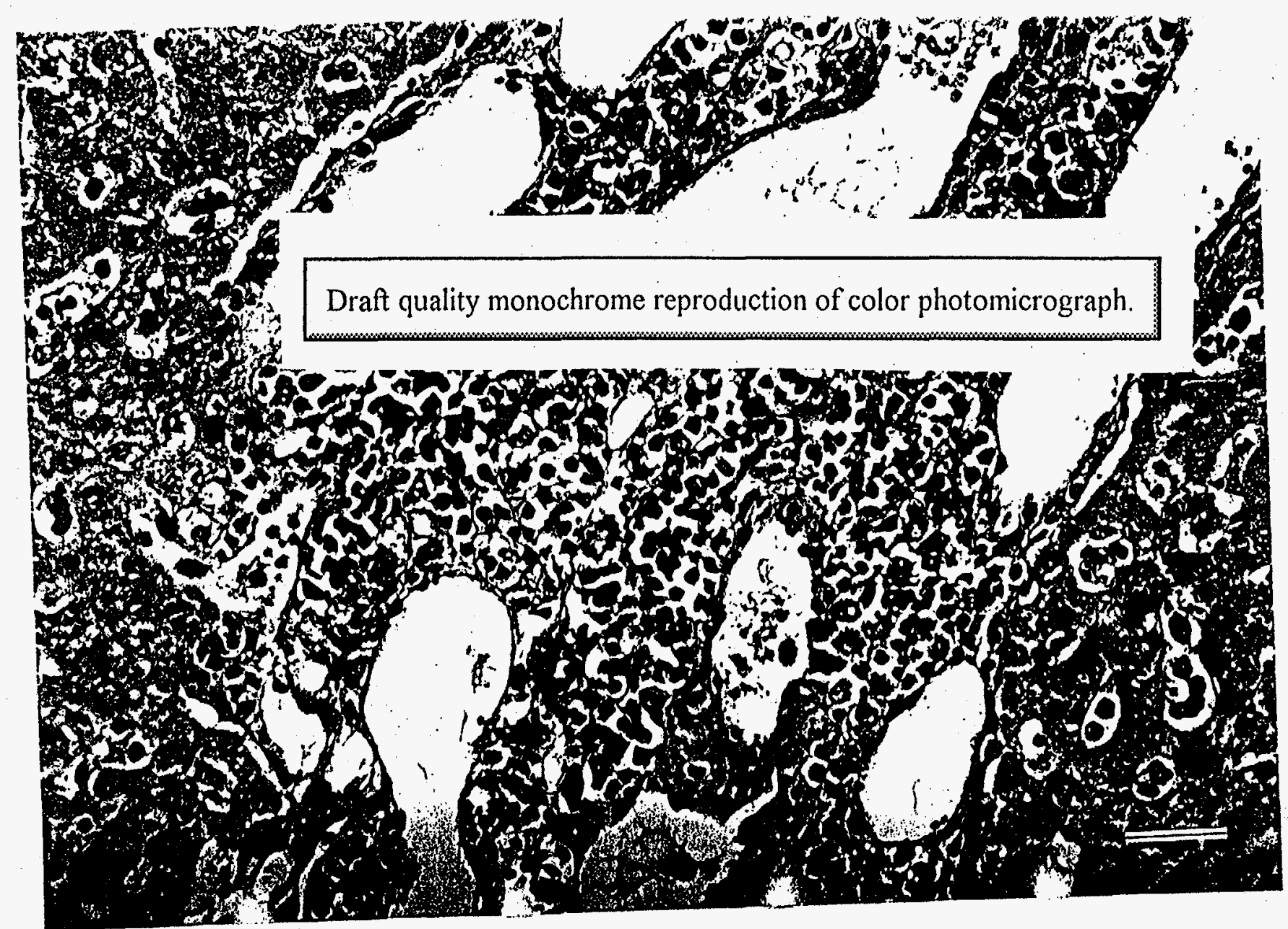

Figure 2.24. EM - Erythremic myelosis. Liver section showing a focus of erythroblastic infiltration (Dog 2976, H\&E). $B a r=40 \mu m$. 
Myeloid Leukemia

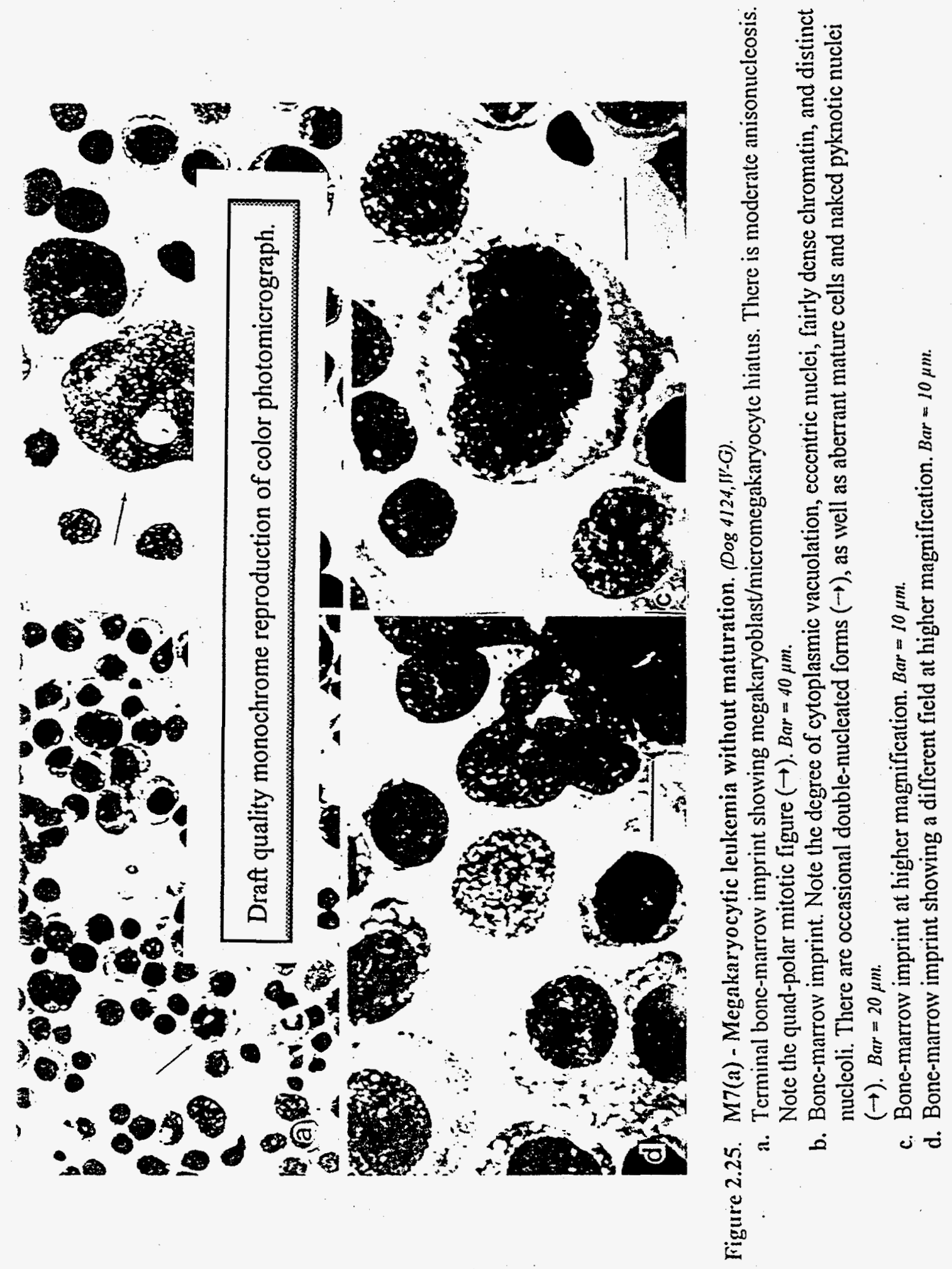




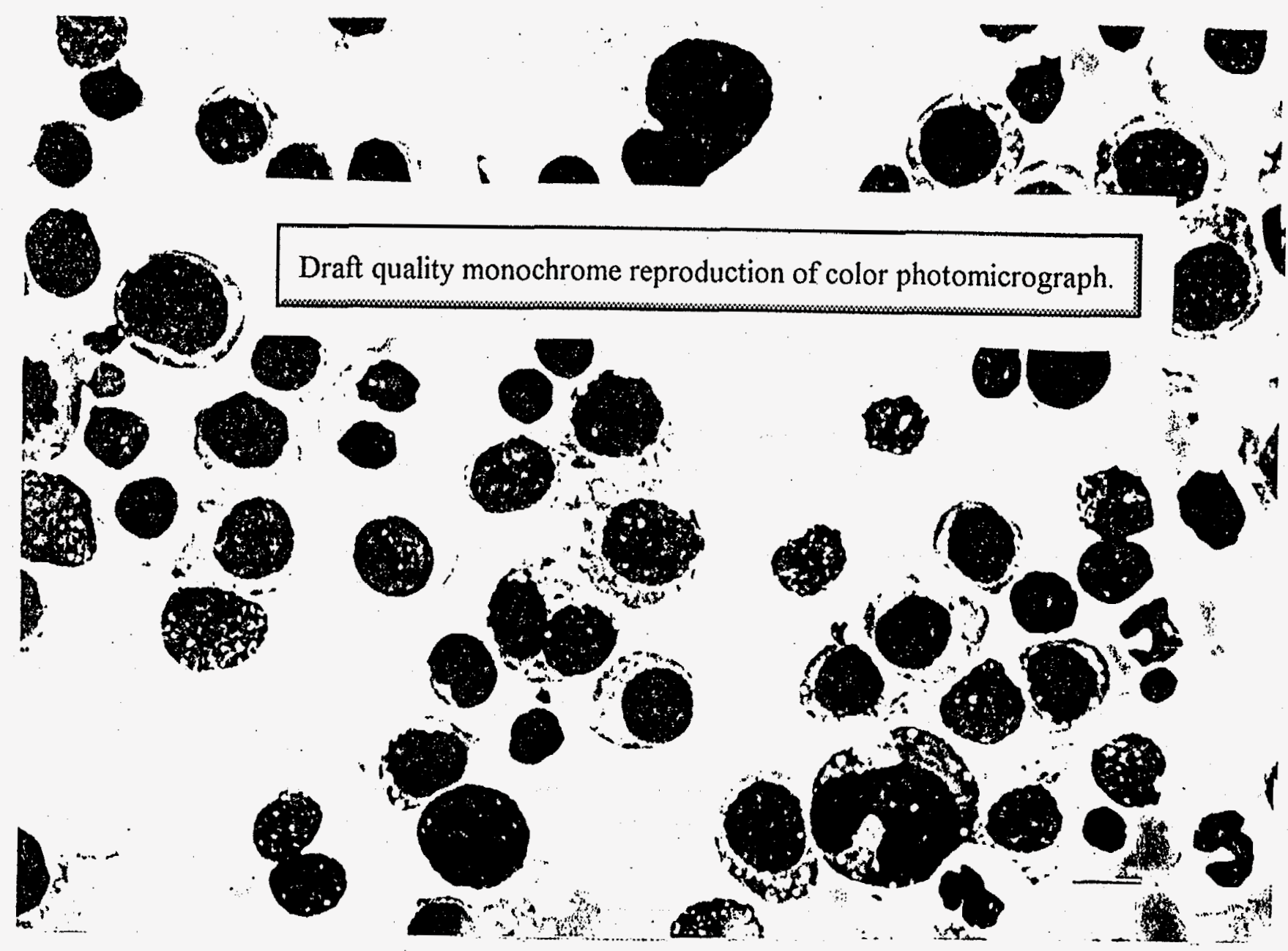

Figure 2.26. M7(a) - Megakaryocytic leukemia without maturation. Larger field from case shown in Figure 2.25, showing a micro-megakaryocyte/megakaryoblast hiatus. Note the marked aniso-cytosis/nucleosis, occasional mitotic figures, double-nucleated cclls and some degree of asynchronous nuclear/cytoplasmic maturation (IV-G). Bar $=10 \mu \mathrm{m}$. 


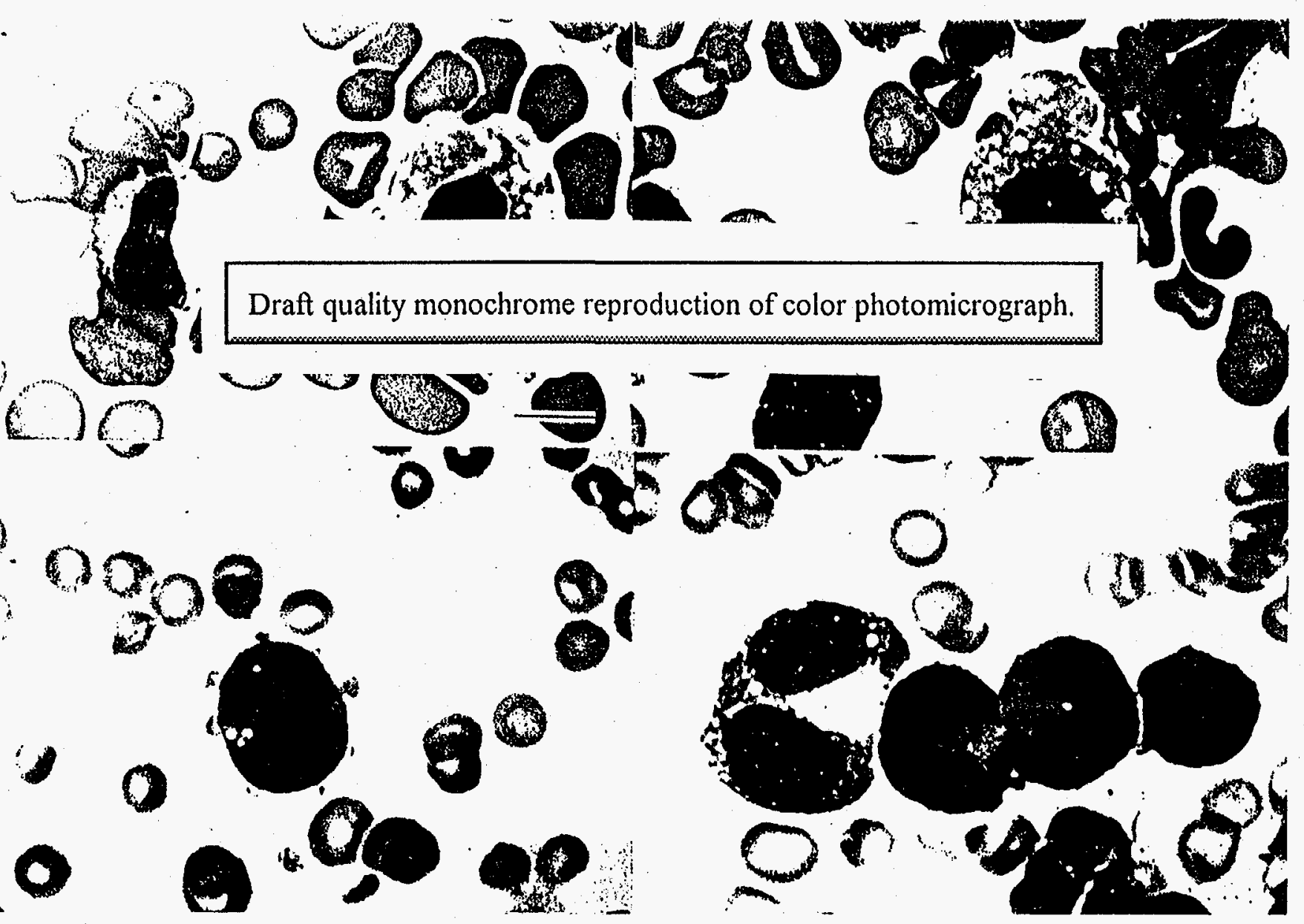

Figure 2.27. M7(b) - Megakaryocytic leukemia with maturation. Peripheral blood buffy cont. Composite of photomicrographs showing various forms of circulating micromegakaryocytes. Note binucleated and mononucleated forms and one cell in mitosis. Also note cytoplasmic buds and giant platclets (Dog $3255, W-G)$, Bar = $10 \mu \mathrm{m}$ for all photomicrographs as shown in composite. 
cloid Leukemia

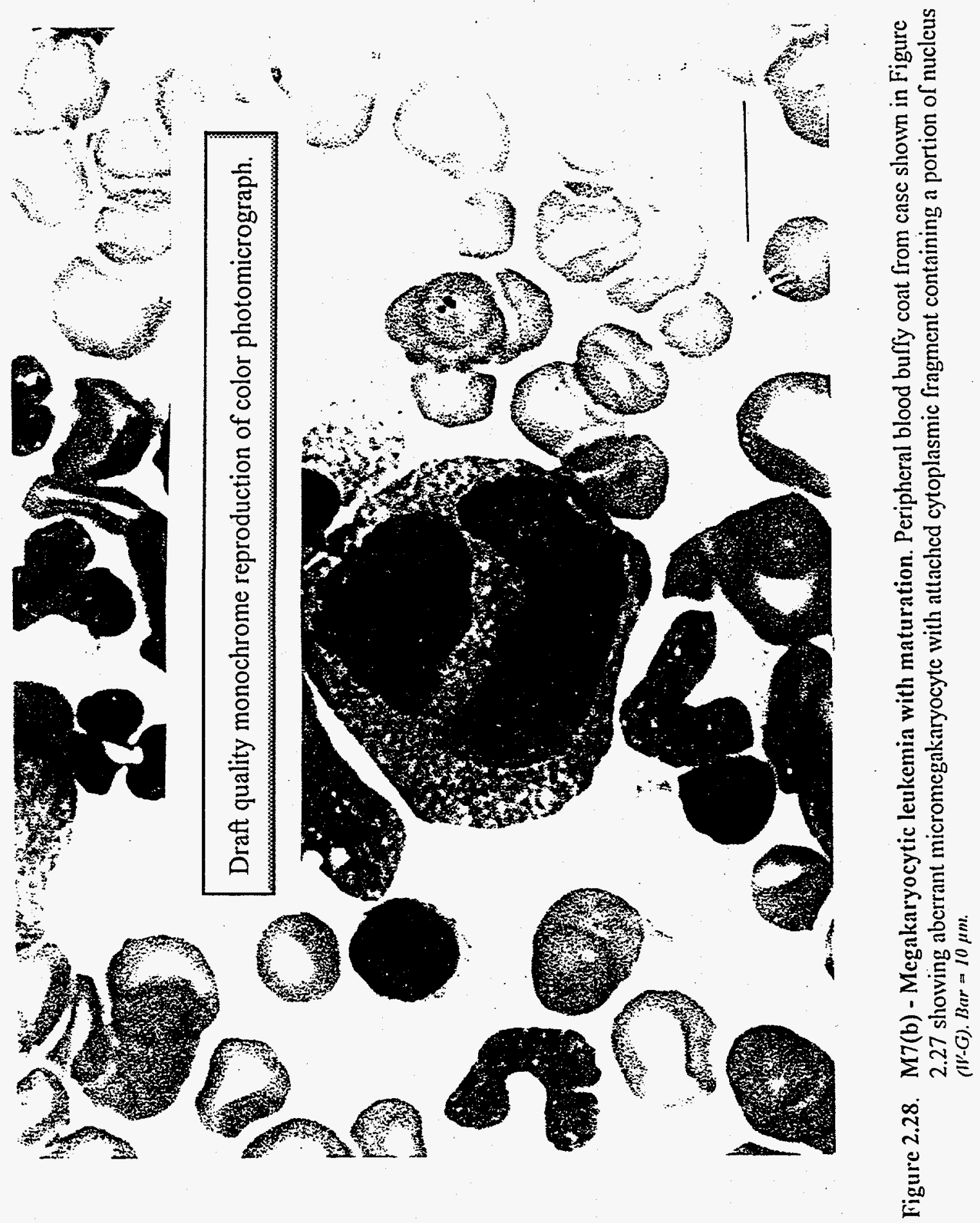


Myeloid Leuḱemiạ

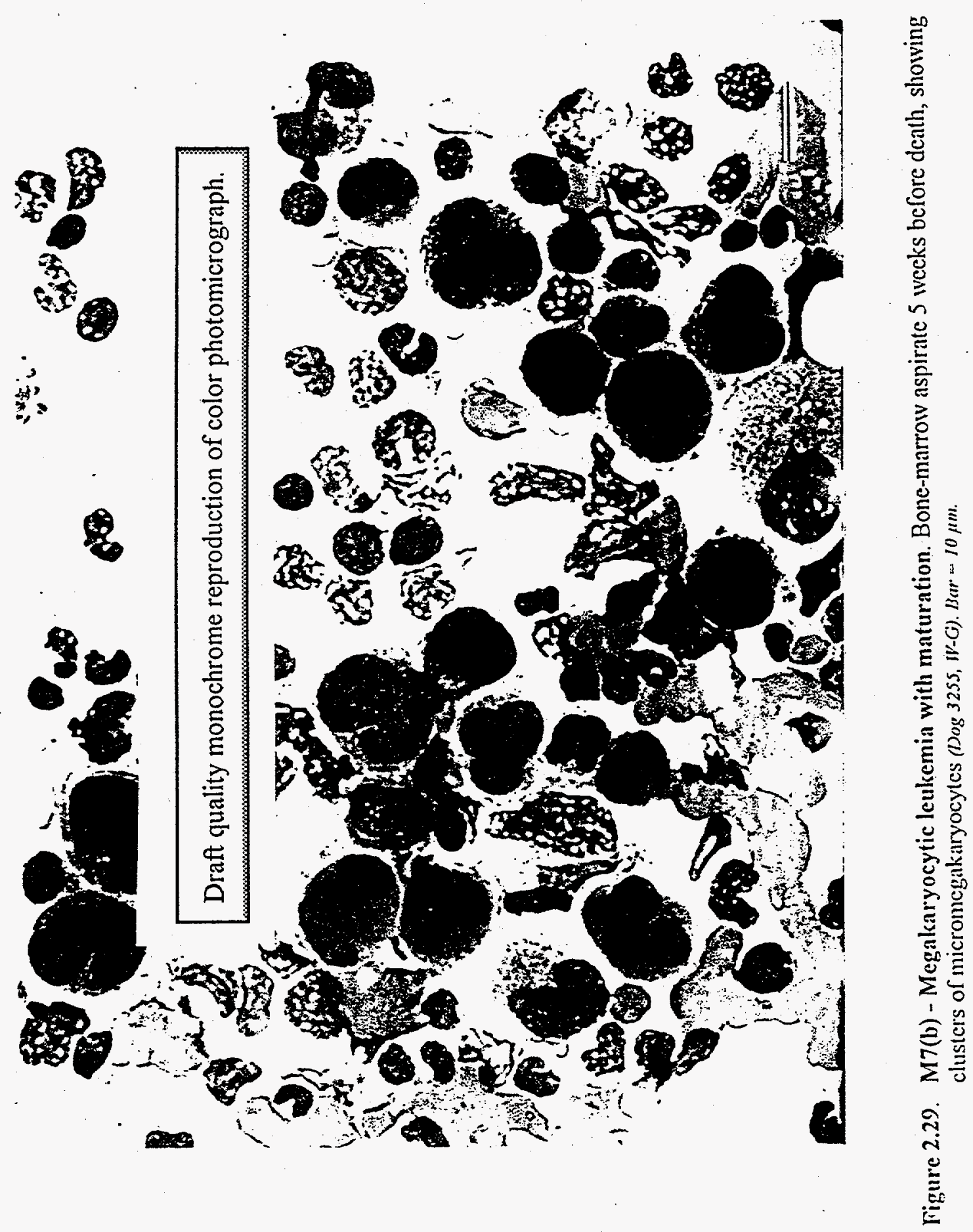



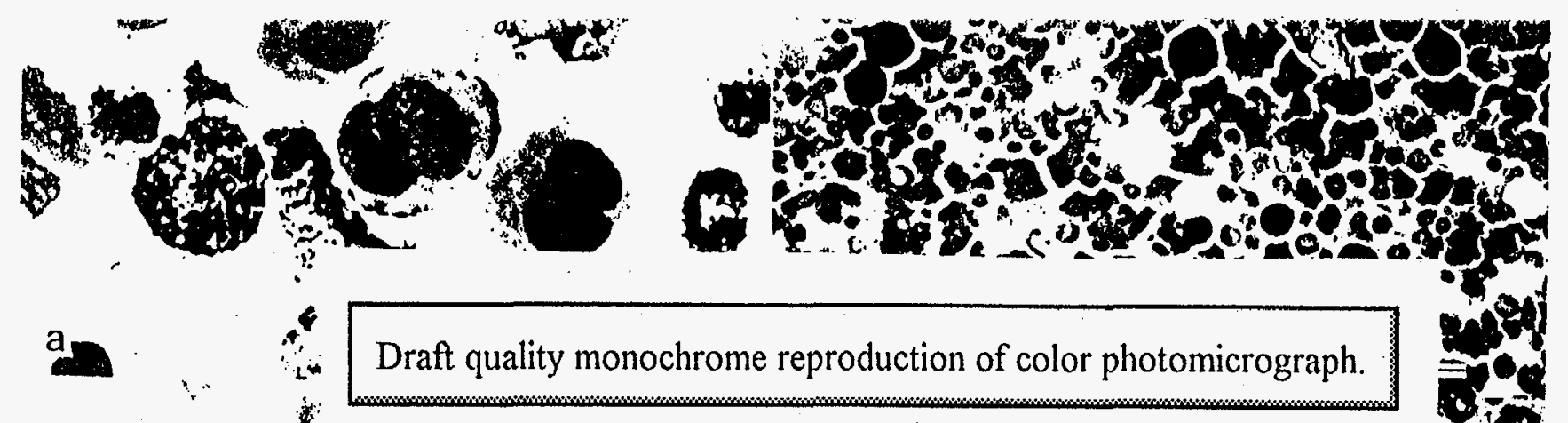

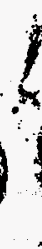
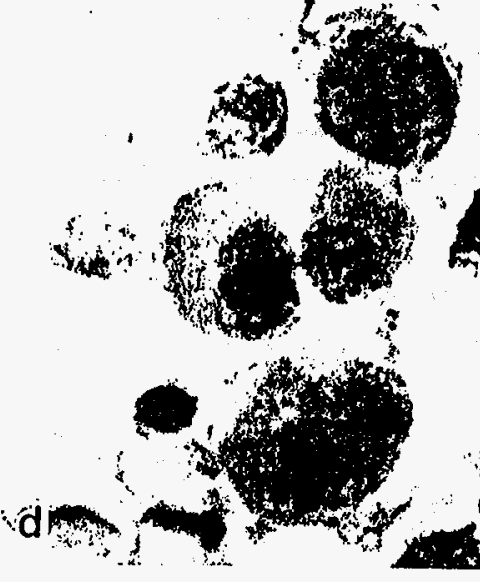

女

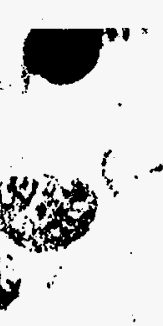

$793^{5} x^{2}$

:

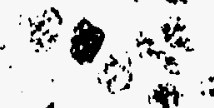

(5)

A

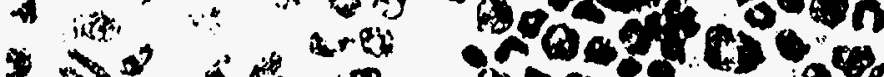

0 (2) $30 \%$

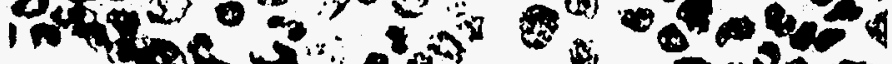

$Y \cdot$

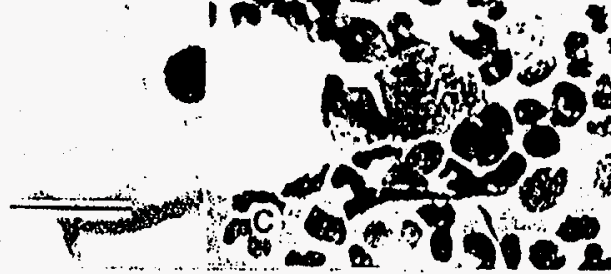

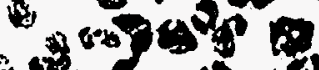
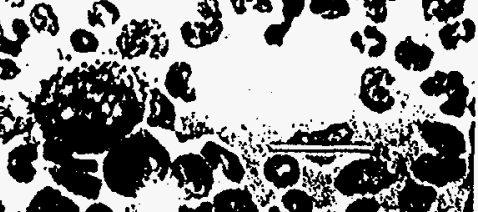

Figure 2.30. M7(b) - Megakaryocytic leukemia with maturation. Bone-marrow aspirate from same case as shown in Figure 2.29. Composite of photomicrographs showing cytochemical characteristics of micromegakaryocytes.
a. myeloperoxidase (-)
b. nonspecific esterase $(+)$
MPO/HH) $\quad B a r=10 \mu \mathrm{m}$.
c. acetylcholinesterase $(+)$
(NSE/HH) Bar $=40 \mu \mathrm{m}$.
d. pcriodic-acid Schiff $(+)$
(ACHE/JH) Bar $=20 \mu \mathrm{m}$.
PAS/HH) $\quad B a r=10 \mu \mathrm{m}$. 

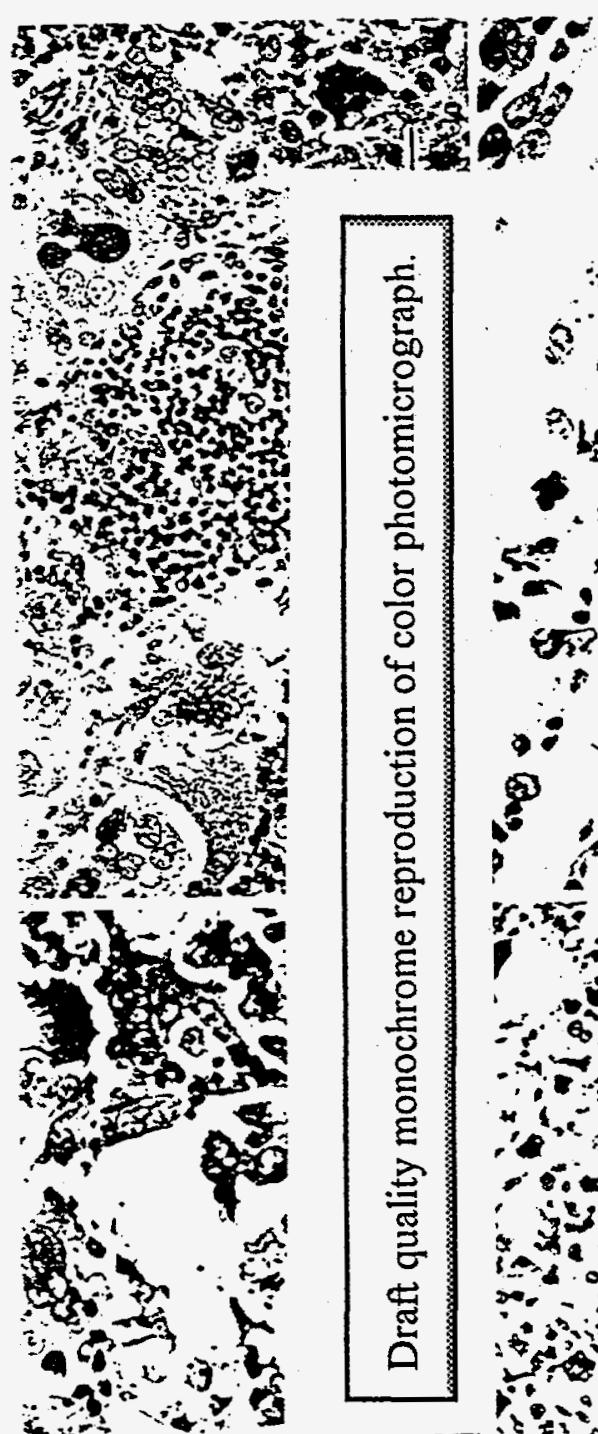

$=$

8

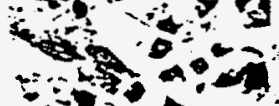

150 a

of

(5) it.

$8 \%$

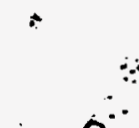
40, $\therefore$ ?

(x) 3 - oses
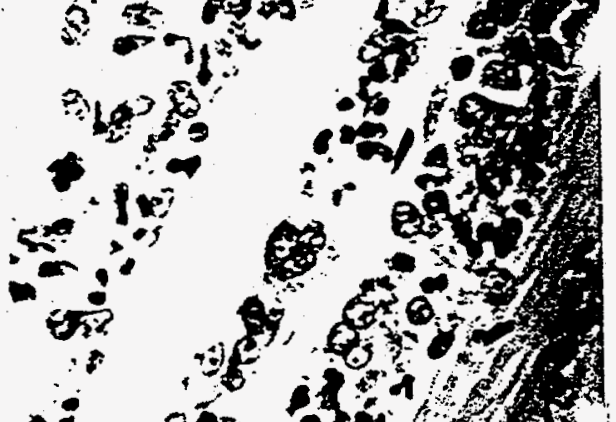

$\because$.

o

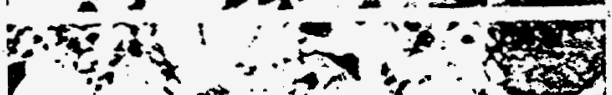

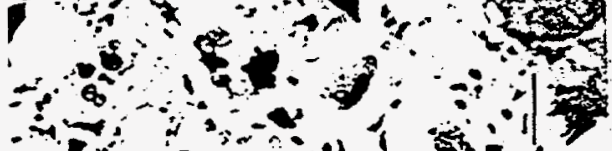
$-10019$

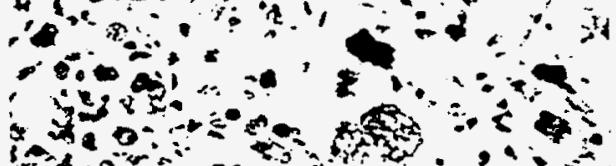

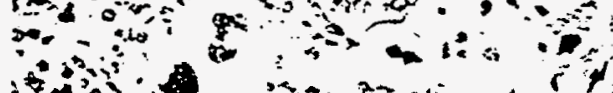
-

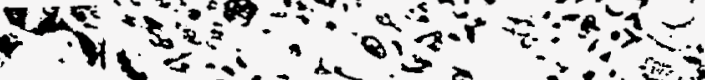

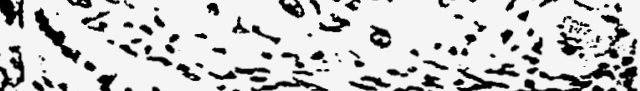
$4 \div 2+\cdots=-20=0$ S. 30.54

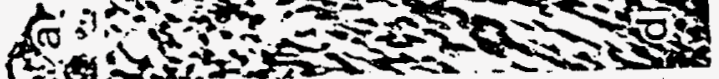

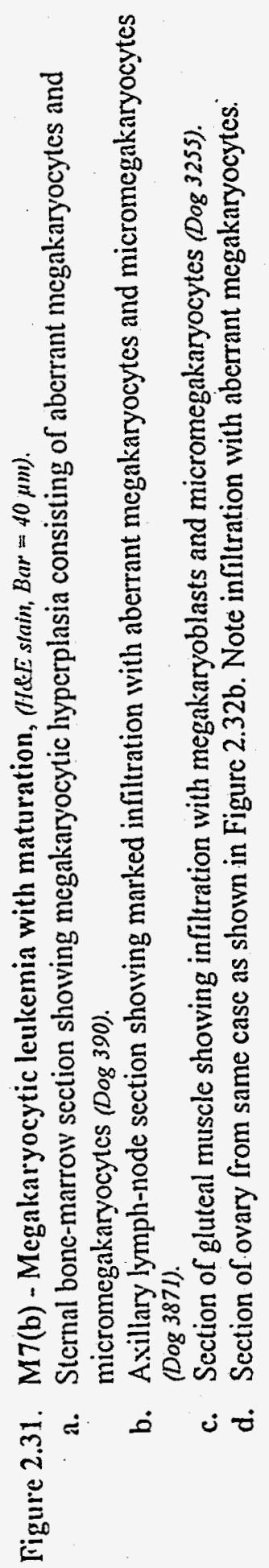




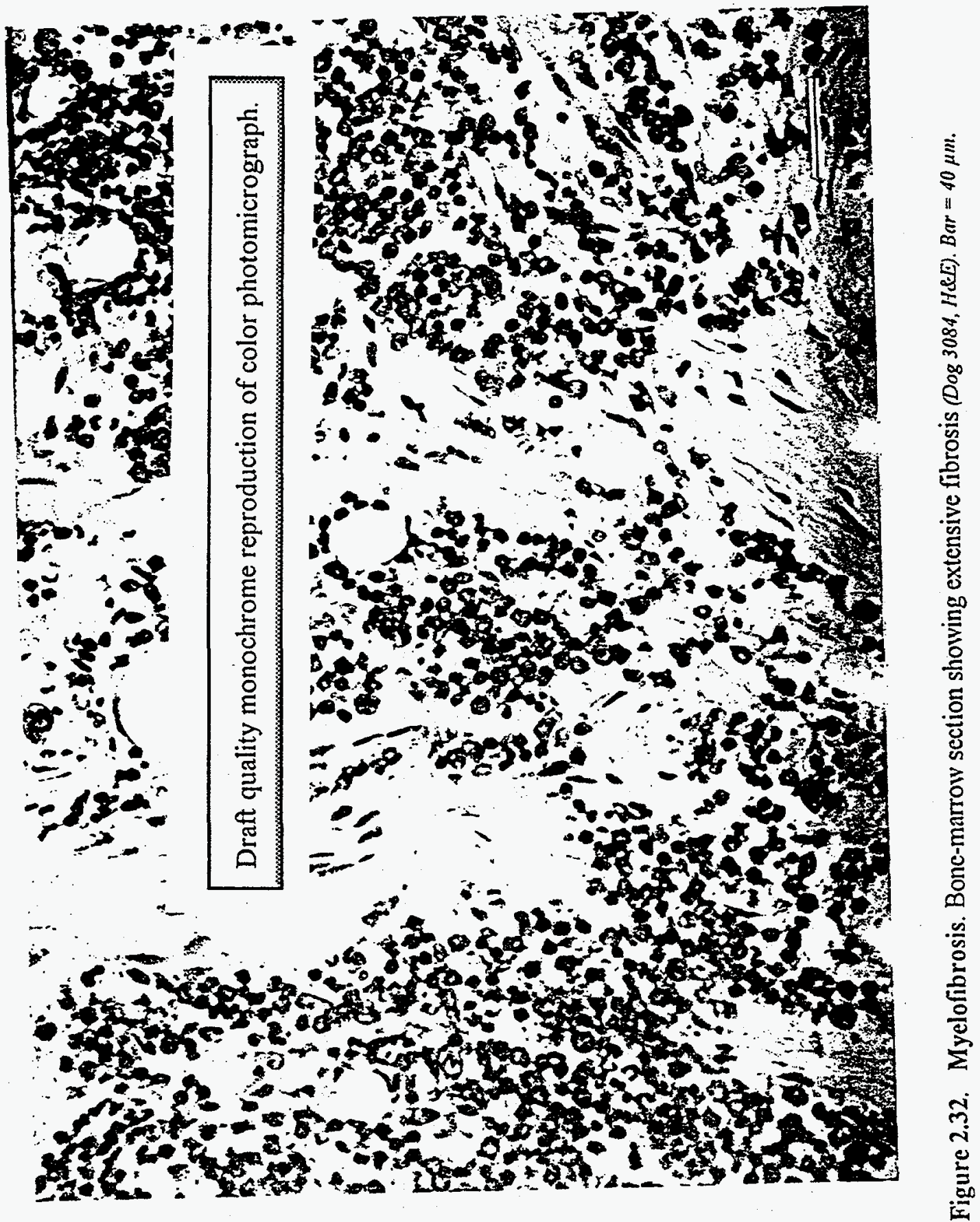



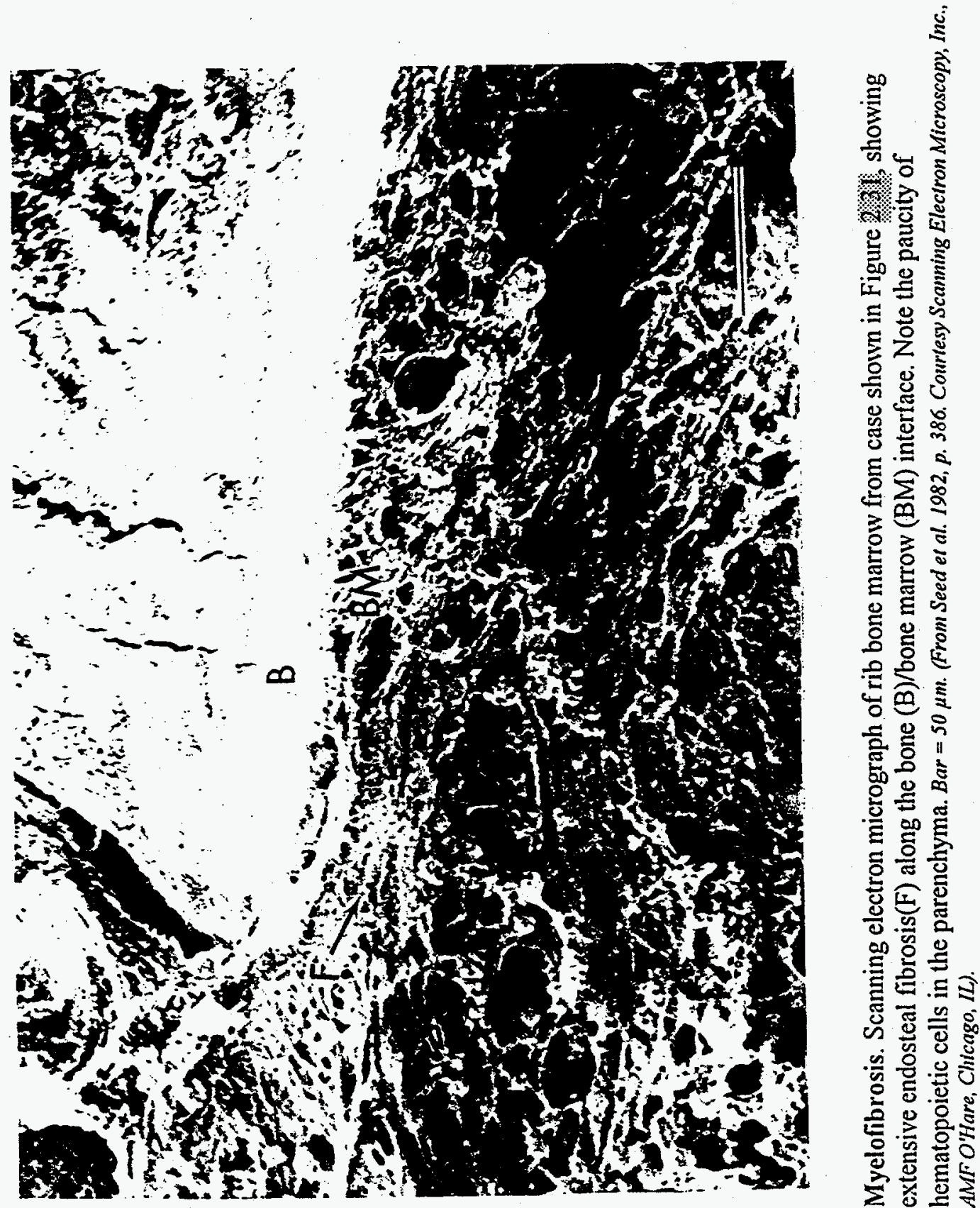

लె 
Myeloid Leukemia
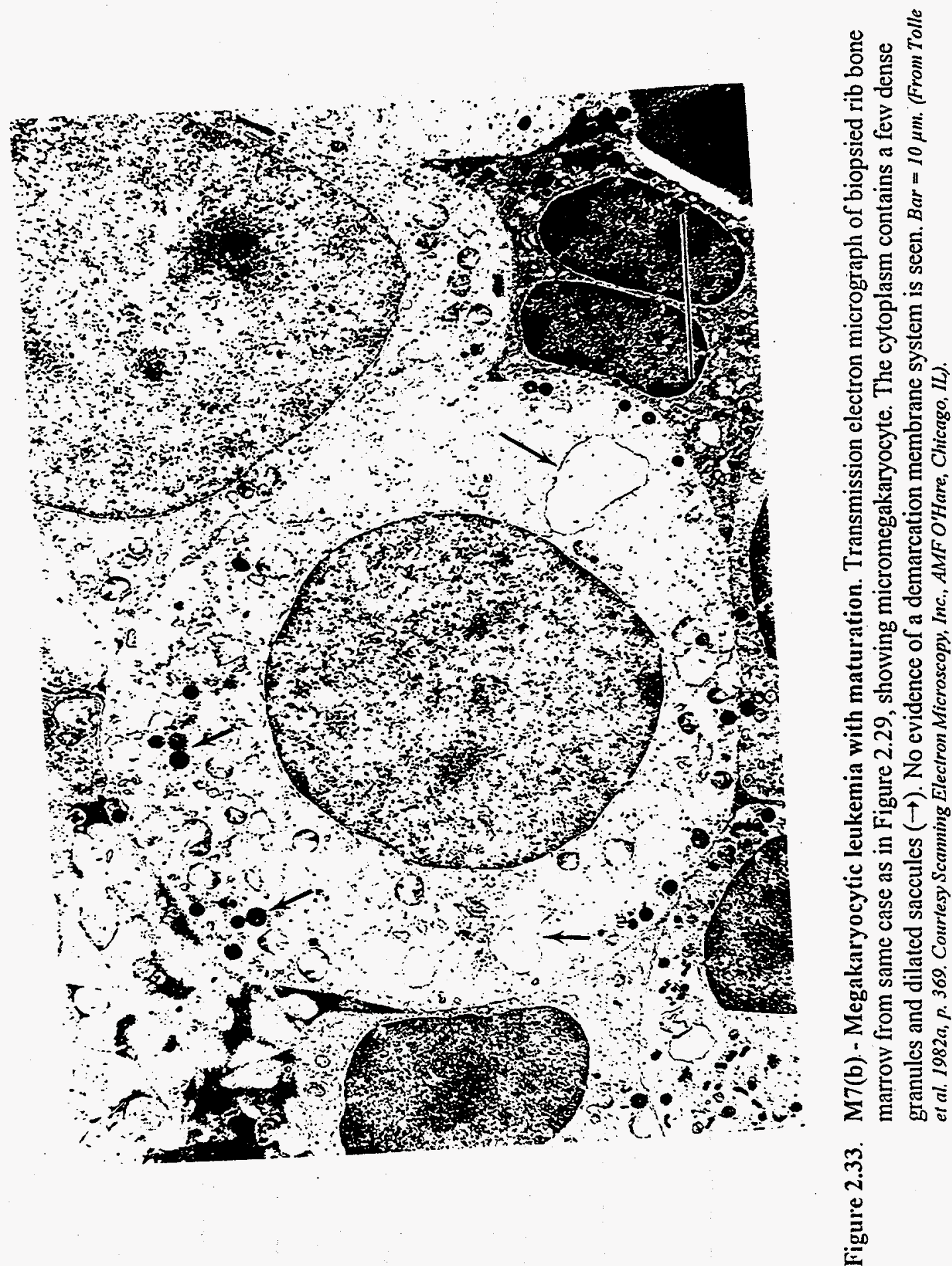
Myeloid Leukemia
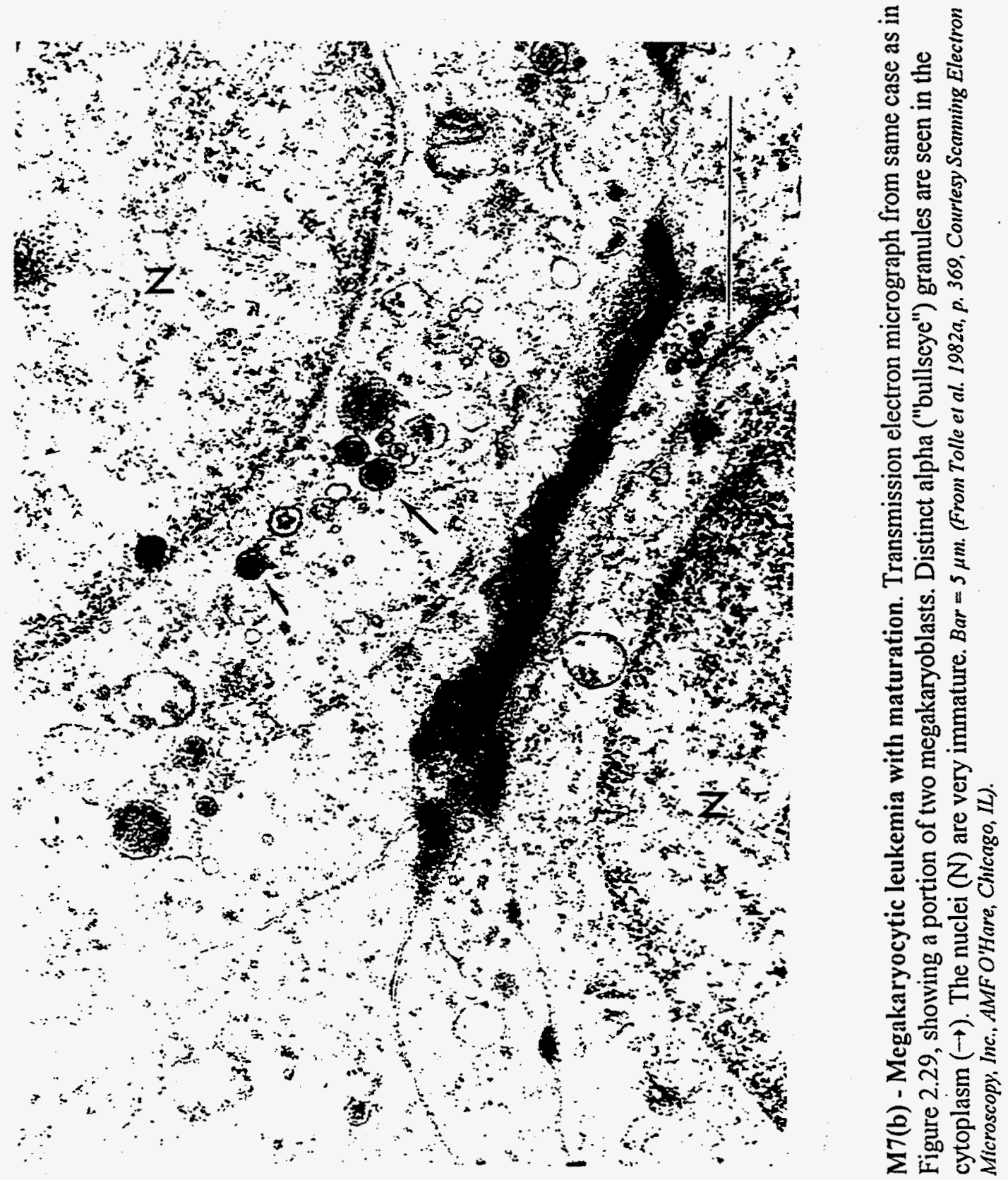

تِّ 
Liver 


\title{
CHAPTER 3: Tumors of the Liver
}

\author{
Glenn N. Taylor \\ University of Utah
}

\section{INTRODUCTION}

The nomenclature of the liver tumors presented in this fascicle was based on the World Health Organization's International Histological Classification of Tumors of Domestic Animals (Ponomarkov and Mackey 1976). The scope of the observations was limited principally to those observed in beagles with variable burdens of ${ }^{293} \mathrm{Pu}$ or ${ }^{241} \mathrm{Am}$. The nomenclature of these lesions is shown in Table 3.1, with an index to illustrations and corresponding SNOMED database codes.

The liver is an important deposition site in dogs for these and some of the other actinide elements translocating from the lungs and from wounds, etc., via the blood vascular system (Mays et al. 1970; Stover, Stevens, and Bruenger 1972; and Taylor et al. 1986). It is also well established that hepatic tissue in humans is also a prime deposition site for some of these radionuclides (Magno, Kauffman, and Groulx 1967; McInroy 1976; Rowland and Durbin 1976; Wegener and Hasenohrl 1983). In beagles, the early deposition pattern was initially quite uniform within the hepatic epithelium, but at later times after injection the translocation of a significant portion of the burden to the Kupffer cells, and ultimately to the central and periportal regions, occurred. Focal nodular hyperplasia was also a significant factor in the intrahepatic distribution. The affinity of the beagle liver for these elements makes it one of the prime target organs in the toxicity schemes of ${ }^{239} \mathrm{Pu},{ }^{241} \mathrm{Am}$, and some of the other actinides and is one of the major reasons for including the liver in this atlas.

Most of the hyperplastic and the neoplastic lesions that are presented also occur "spontaneously" and are not unique to a radiation etiology. Nevertheless, the dose-response relationships indicated that most of the tumors presented here were radiation-induced.

Injected dosages have been indicated for each respective animal in microocuries per kilogram of body weights, but this is not meant to imply a relationship between a given dose and a given tumor type. For example, some of the specific tumors were observed over a wide spectrum of treatment levels.

It should also be noted that this chapter presents only the primary hepatic tumors that have been observed, to date, in beagle studies at several DOE laboratories. Thus, additions to this chapter will probably be necessary as data are received from experiments that are still in progress or that are being conducted in other facilities. 
Table 3.1. Liver Neoplasia Nomenclature, Index of Figures, and SNODOG Database Codes.

\begin{tabular}{|c|c|c|}
\hline Nomenclature & Figure(s) & $\begin{array}{c}\text { SNODOG } \\
\text { Morphology } \\
\text { Code } \\
\end{array}$ \\
\hline \multicolumn{3}{|l|}{ Non-neoplastic } \\
\hline Atrophy & 3.1 & M580000 \\
\hline Nodular hyperplasia & $3.2-5$ & M720300 \\
\hline \multicolumn{3}{|l|}{ Neoplastic } \\
\hline Benign intrahepatic cystadenoma & 3.6 & M844000 \\
\hline Intrahepatic bile duct adenoma & $3.7-9$ & M816000 \\
\hline Hepatic cell carcinoma & $3.10-11$ & M817030 \\
\hline Intrahepatic cholangiocarcinoma & $3.12-15$ & M816030 \\
\hline Mesothelioma & $3.16-17$ & M905030 \\
\hline Intrahepatic carcinoid & 3.18 & M824030 \\
\hline Hemangiosarcoma & 3.19 & M912030 \\
\hline Intrahepatic fibrosarcoma & 3.20 & M881030 \\
\hline Myxoma & 3.21 & M884000 \\
\hline Myxosarcoma & 3.22 & M884030 \\
\hline Mast cell sarcoma & 3.23 & M974030 \\
\hline
\end{tabular}




\section{REFERENCES}

Magno, P. J., P. E. Kauffman, and P. R. Groulx. 1967. Plutonium in environmental and biological media. Health Phys. 13:1325-1330.

Mays, C. W., G. N. Taylor, W. S. S. Jee, and T. F. Doughtery. 1970. Speculated risk to bone and liver from ${ }^{239} \mathrm{Pu}$. Health Phys. 19:601-610.

McInroy, J. F. 1976. The Los Alamos Scientific Laboratory's Human Autopsy Tissue Study, pp. 249-270. In: The Health Effects of Plutonium and Radium, W. S. S. Jee, Ed. J. W. Press, Salt Lake City, UT.

Ponomarkov, V. and L. J. Mackey. 1976. XIII. Tumours of the Liver and Biliary System, pp. 187-194. In: The Bulletin of the World Health Organization, International Histological Classification of Tumours of Domestic Animals, 53, No. 2-3, WHO, Geneva, Switzerland.

Rowland, R. E., and P. W. Durbin. 1976. Survival, Causes of Death, and Estimated Tissue Doses in a Group of Human Beings Injected with Plutonium. pp. 329-342. In: The Health Effects of Plutonium and Radium, W. S. S. Jee, Ed. J. W. Press, Salt Lake City, UT.

Selye, H., 1965. The Mast Cells, p. 91. Baltimore, Waverly Press, Inc., MD.

Stover, B. J., W. Stevens, and F. W. Bruenger. Chemical Associations of ${ }^{239} \mathrm{Pu}$ (IV) and ${ }^{241} \mathrm{Am}(\mathrm{III})$ in Blood, Liver, and Thyroid, pp. 129-169. In: Radiobiology of Plutonium, B. J. Stover and W. S. S. Jee, Eds. The J. W. Press, Salt Lake City, UT.

Taylor, G. N., C. W. Mays, M. E. Wrenn, L. Shabestari, and R. D. Lloyd. 1986. Incidence of Liver Tumors in Beagles with Body Burdens of ${ }^{239} \mathrm{Pu}$ or ${ }^{241} \mathrm{Am}, \mathrm{pp}$. 268-285. In: Life-Span Radiation Effects Studies in Animals: What Can They Tell Us? R. C. Thompson and J. A. Mahaffy, Eds. CONF 830951, NTIS, Springfield, VA.

Wegener, K. and K Hasenohrl. 1983. Recent results of the German Thorotrast study:

Pathoanatomical changes in animal experiments and comparison to human thorotrastosis. Health Phys. 44 (Suppl. 1): 307-316. 


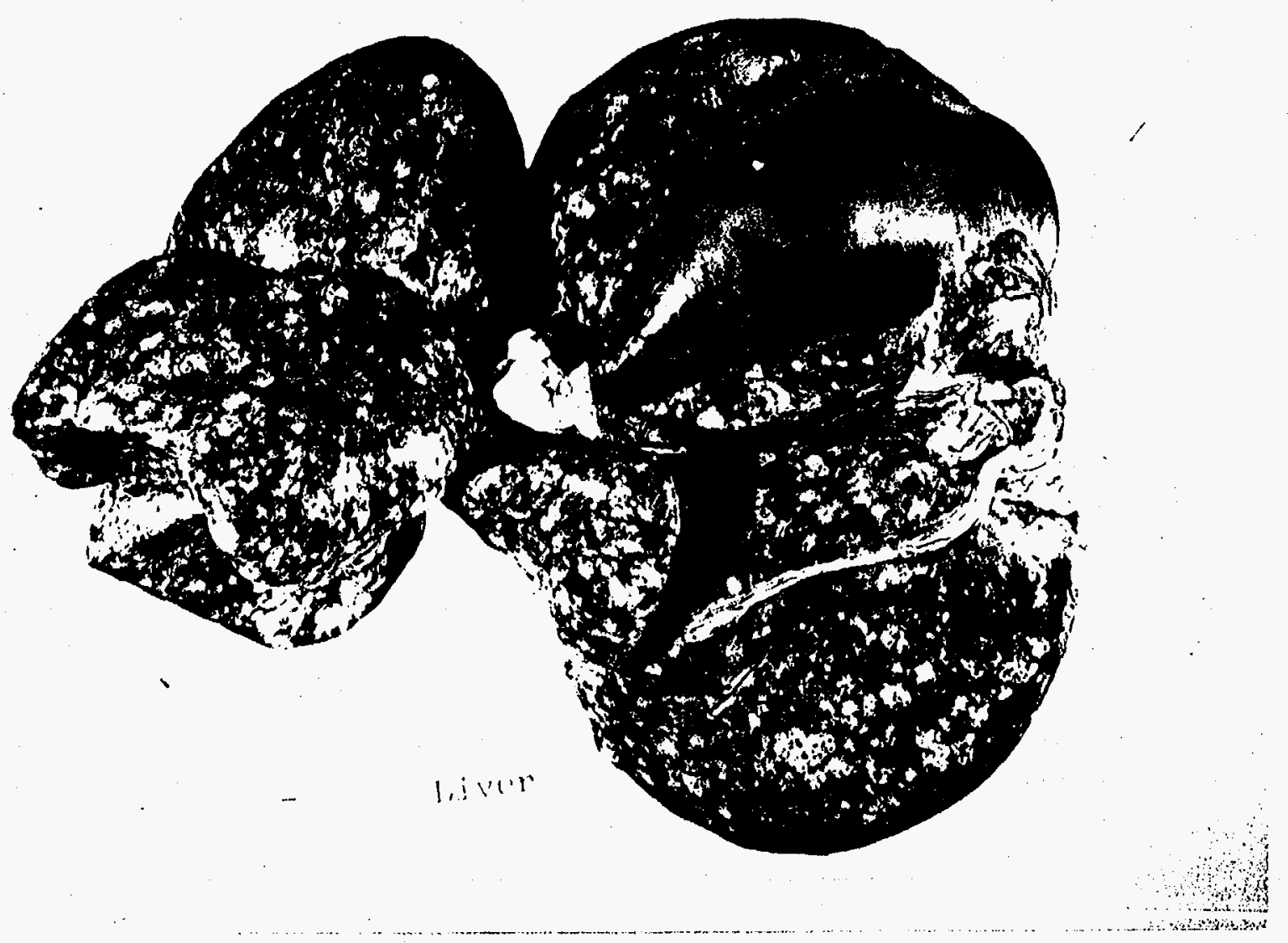

Figure 3.1 Radiation-induced ( $\mathrm{Pu}$ ) hepatic atrophy, degeneration, and hyperplasia, which were secn at the highest dose lcvel studied $(3 \mu \mathrm{Ci} / \mathrm{kg})$. Although significant hepatocyte hyperplasia was present in this liver, the replacement of the parenchyma did not keep pace with necrosis, and the mass of this liver was about one-third of normal. Also, the normal sinusoidal architecture was not maintained in the regenerated tissue, producing marked portal hypertension and ascites. Beagle: T46PS (Male); Age: 3.15y; Time after injection: 2.01 y; Treatment:3.01 $\mu \mathrm{Ci}^{239} \mathrm{Pu} / \mathrm{kg}$. 


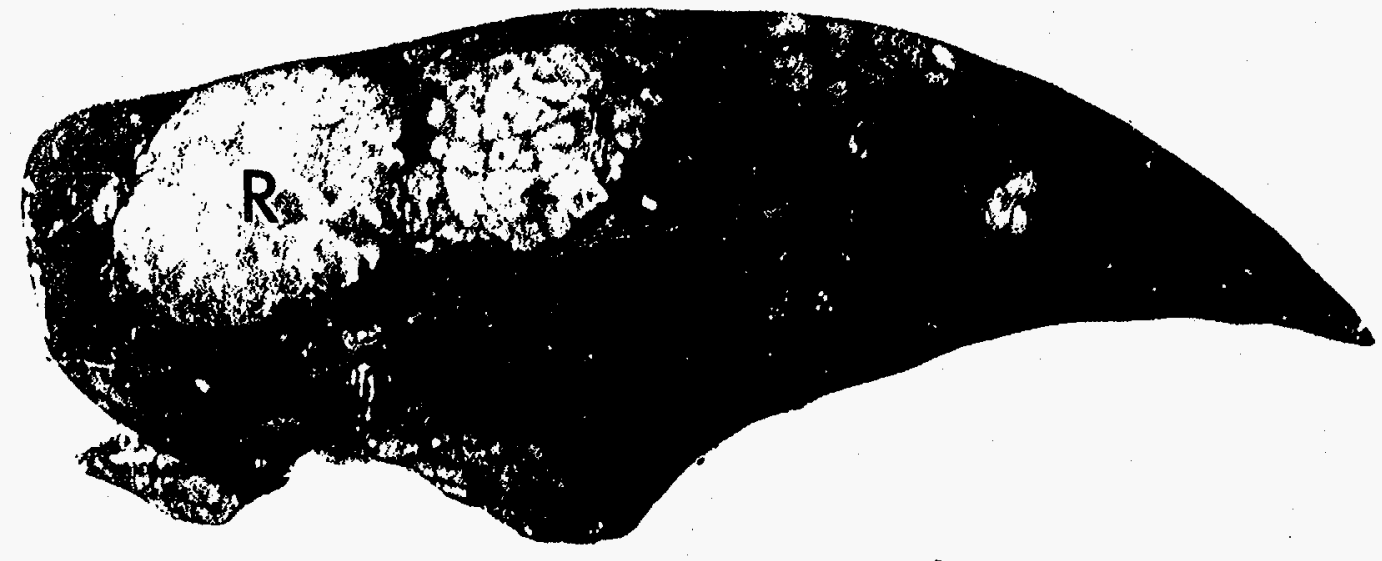

Figure 3.2 Nonencapsulated focal hyperplasia $(R)$ within acini and contingent with normal liver parenchyma. These were subunits of a grossly apparent nodule, shown in Figure 3.2. Such focal hyperplasia produced distortion and enlargement of the involved acini, but the general acinar pattern was usually retained, distinguishing such hyperplasia from hepatic cell adenomas. Although not definitely established, there was evidence that clusters of hyperplastic acini tended to be oriented around a given branch of the portal tree, producing the much larger grossly apparent nodule. Fibrosis was generally not present. Beagle: FoP1.7 (Female); Age: 8.92 y; Time after injection: 6.85 y: Treatment: $0.0485 \mu \mathrm{Ci} \quad \mathrm{Pu} / \mathrm{kg}$ 

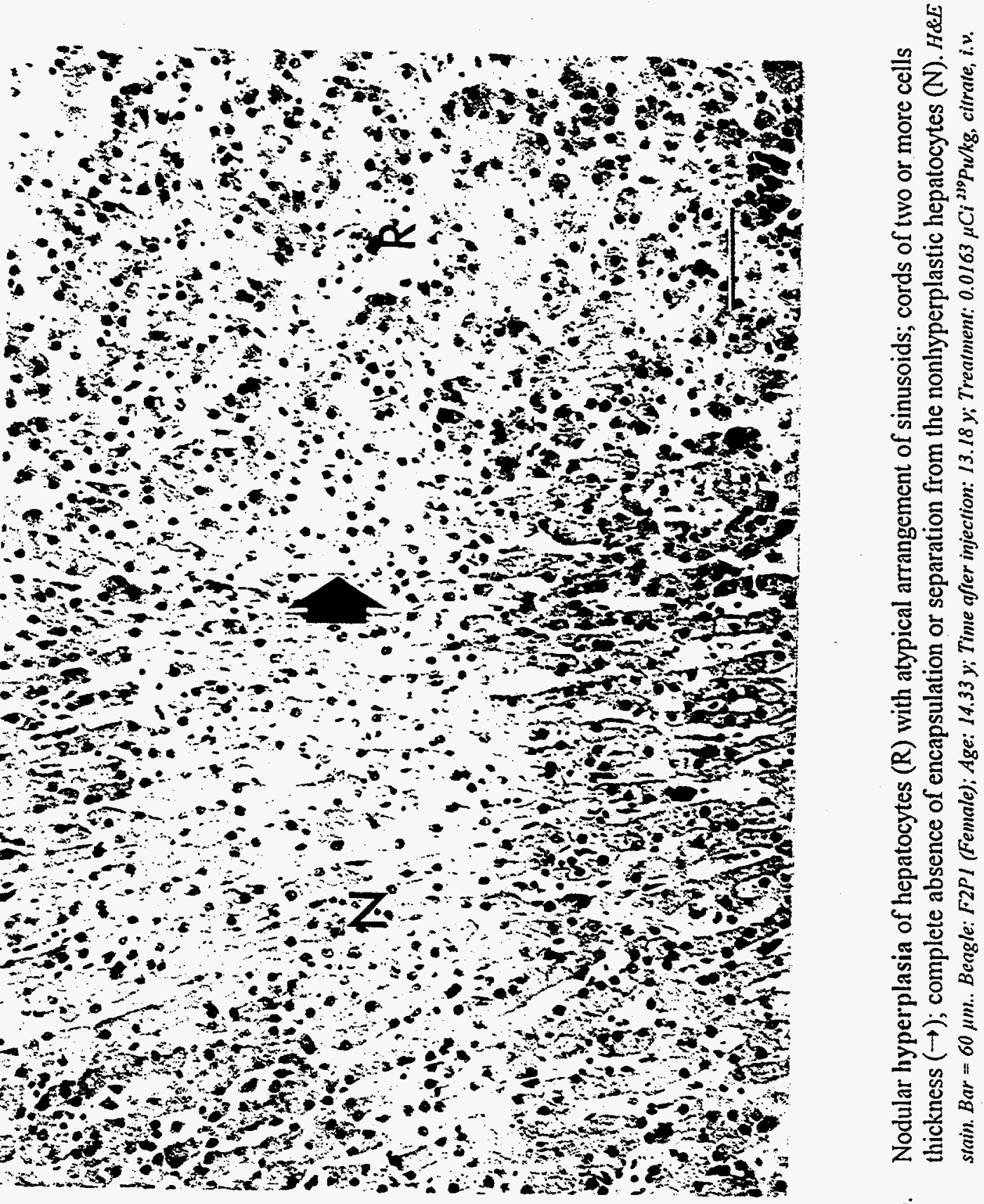

幽 


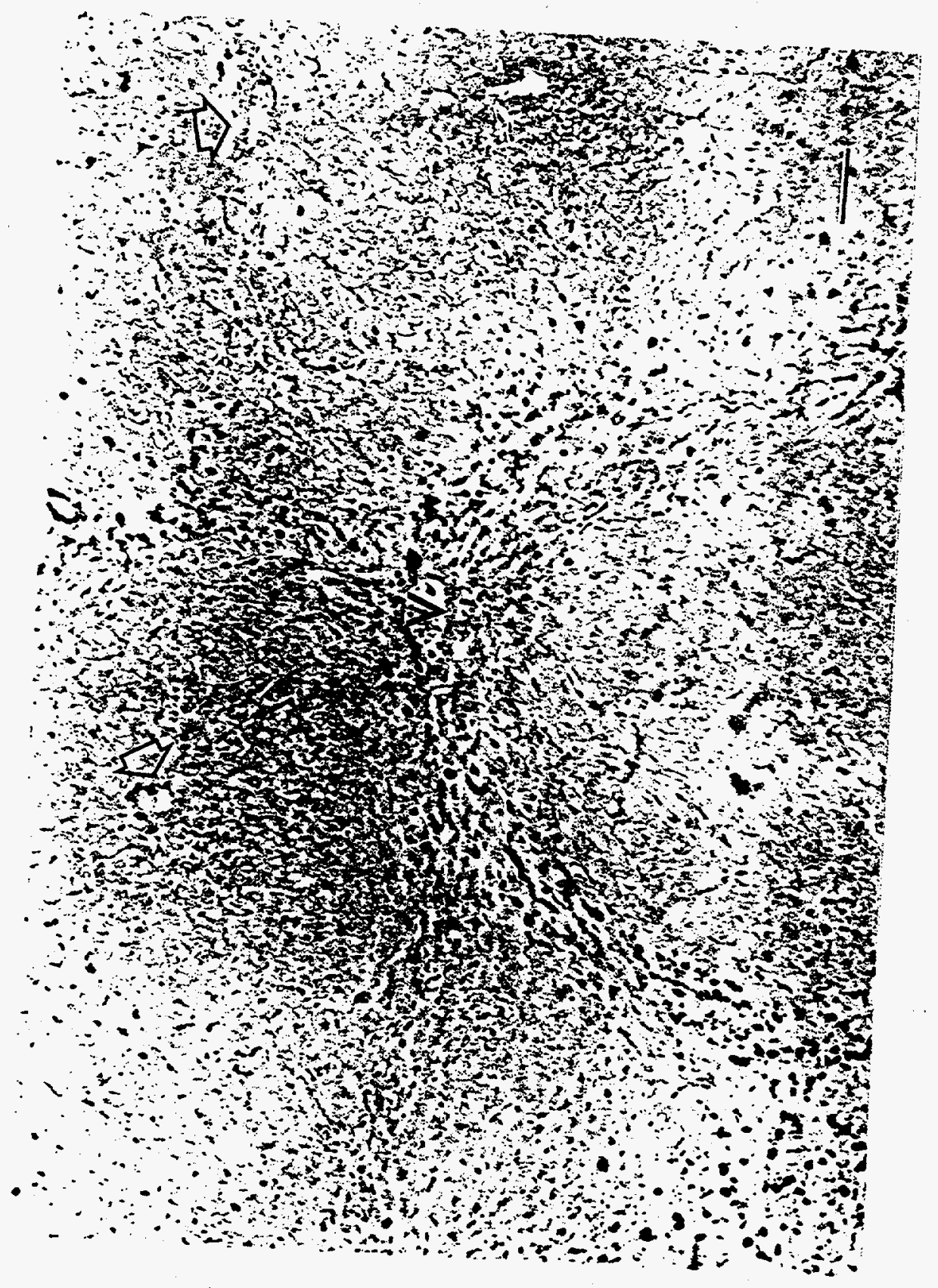

$\stackrel{0}{\Xi}$

$\sum_{0}^{2}$

कू है

太

โ穿

跑

次

눙 을 은

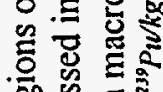

कo

通泀

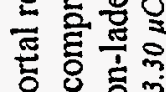

을.

己氙

$\stackrel{\varrho}{=}$ 色

픙.음

空

. 웡

웡

을 일

可

ㄷㅡㄹㅀㅡㄹ

ธี

을

跣

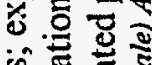

远 䒕

엉으은

氖

远哭

.

ज्ञ

은 놓

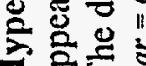

空总

نั 


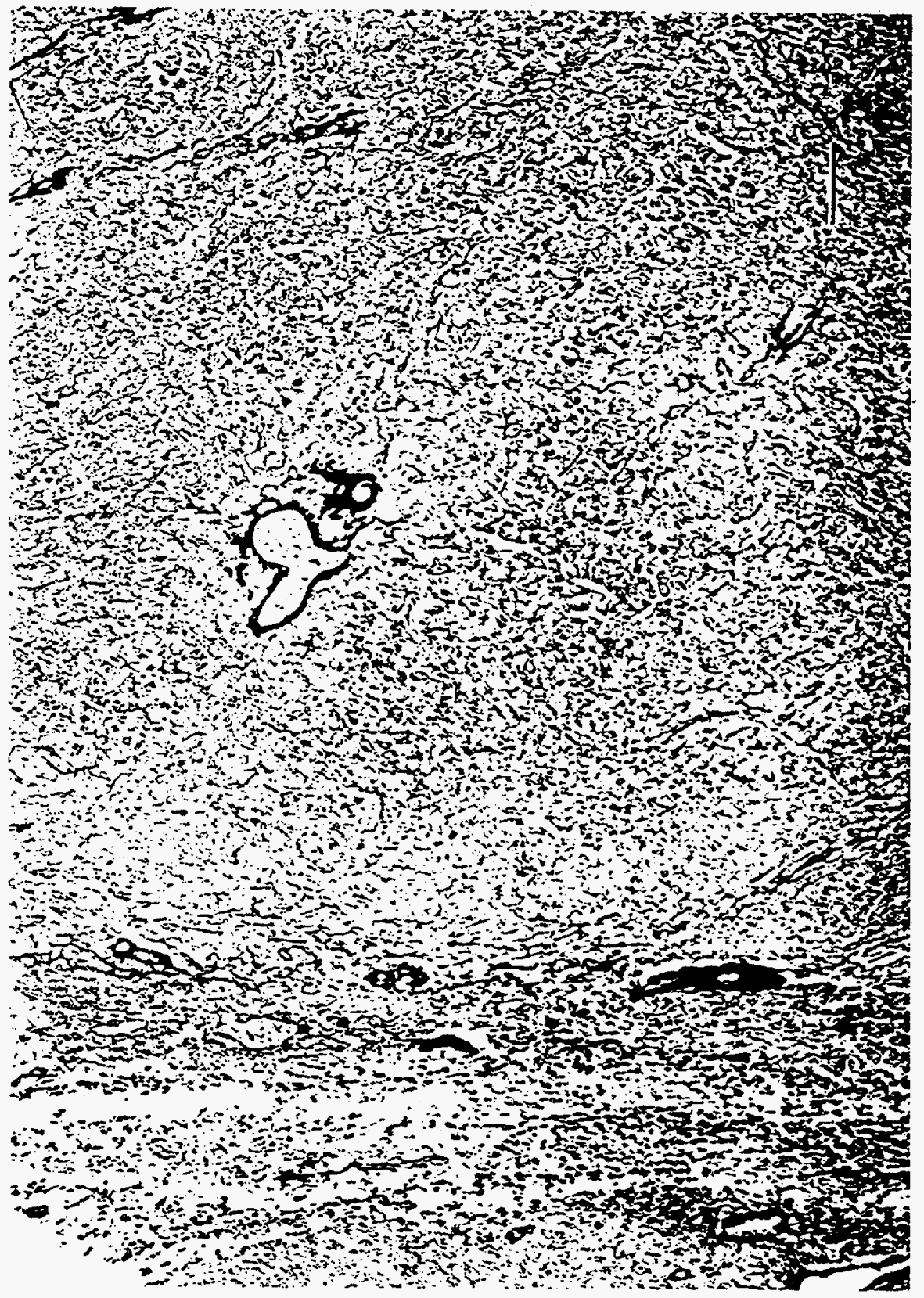

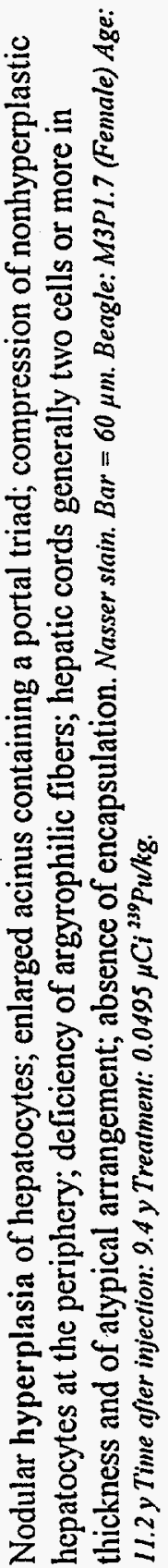

ñ 


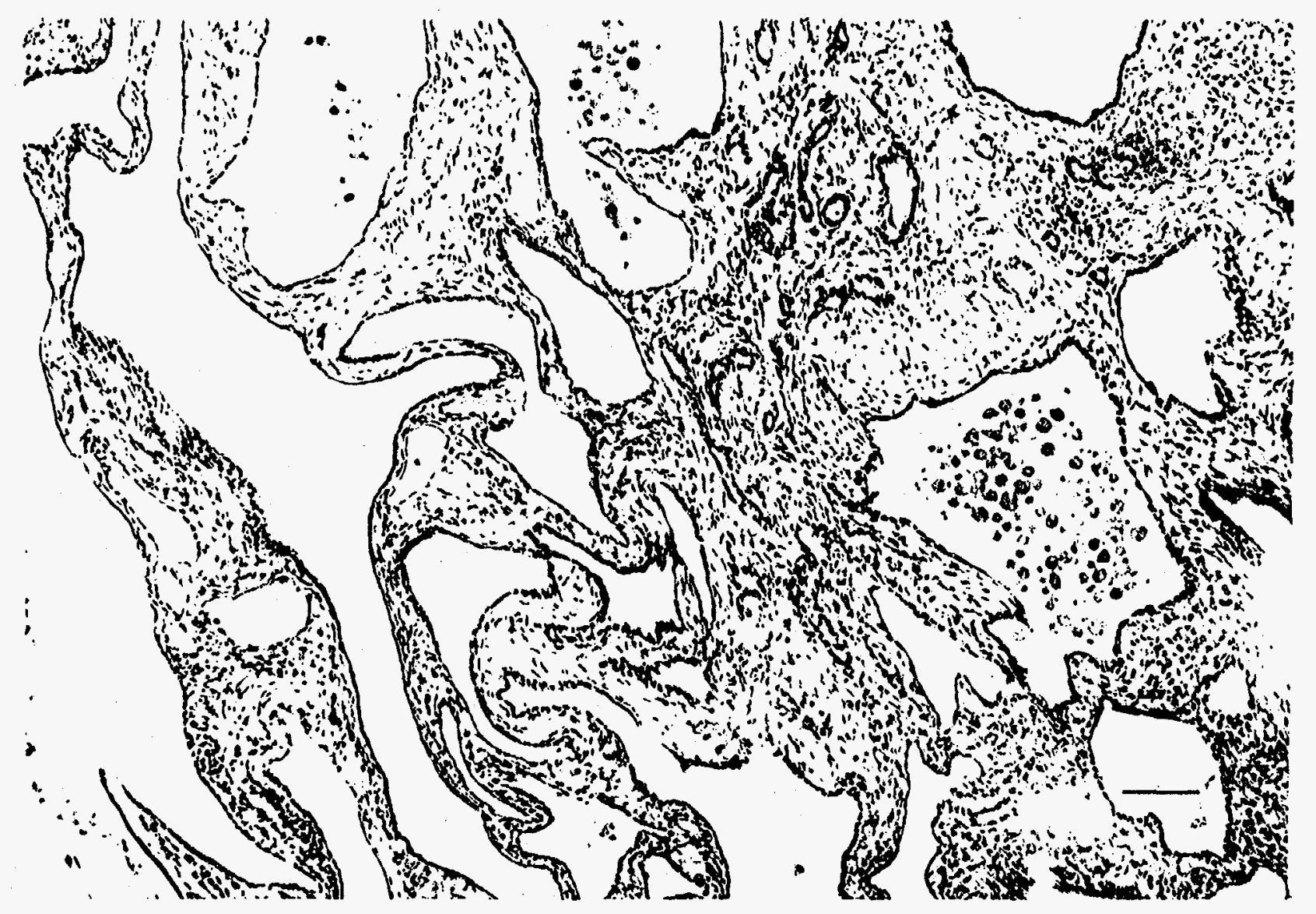

Figure 3.6 Benign intrahepatic cystadenoma. Multiloculated cystic cavities are lined by cuboidal to low-cuboidal epithelium and contain watery or mucinous exudate with monocytic infiltrates. In some instances the individual compartments vary in size from less than $1 \mathrm{~mm}$ to over $10 \mathrm{~cm}$ in diameter. The fibrous stroma is abundant and is focally infiltrated with leukocytes. Mitotic figures were not seen. $H \& E$ stain. Bar $=60 \mu \mathrm{m}$. Beagle: FJ0IP0.5Y (Female) Age: 11.8 y Time after injection: 11.5 y Treatment: $0.00617 \mu \mathrm{Ci} i^{239} \mathrm{P} \mathrm{w} / \mathrm{kg}$. 


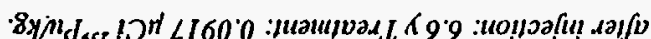

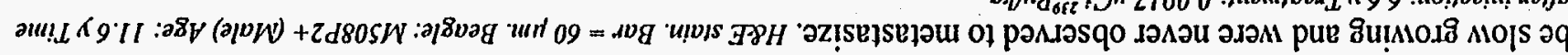

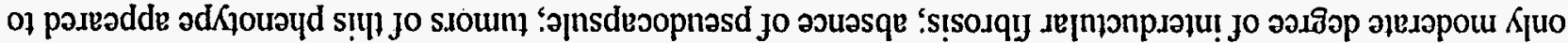

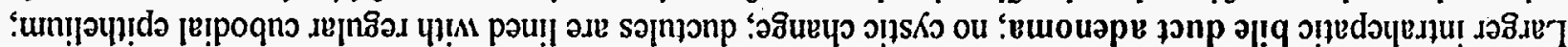

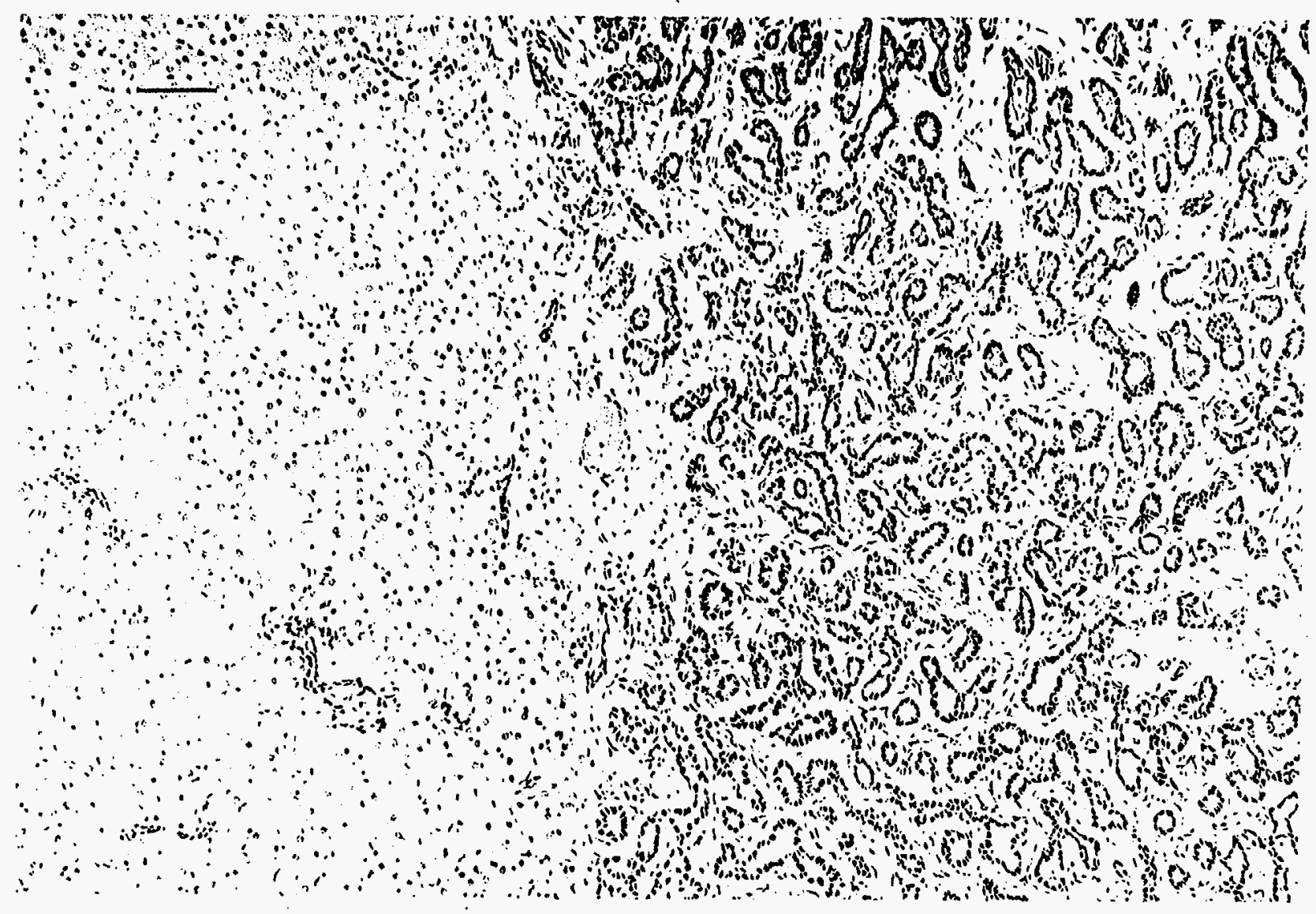



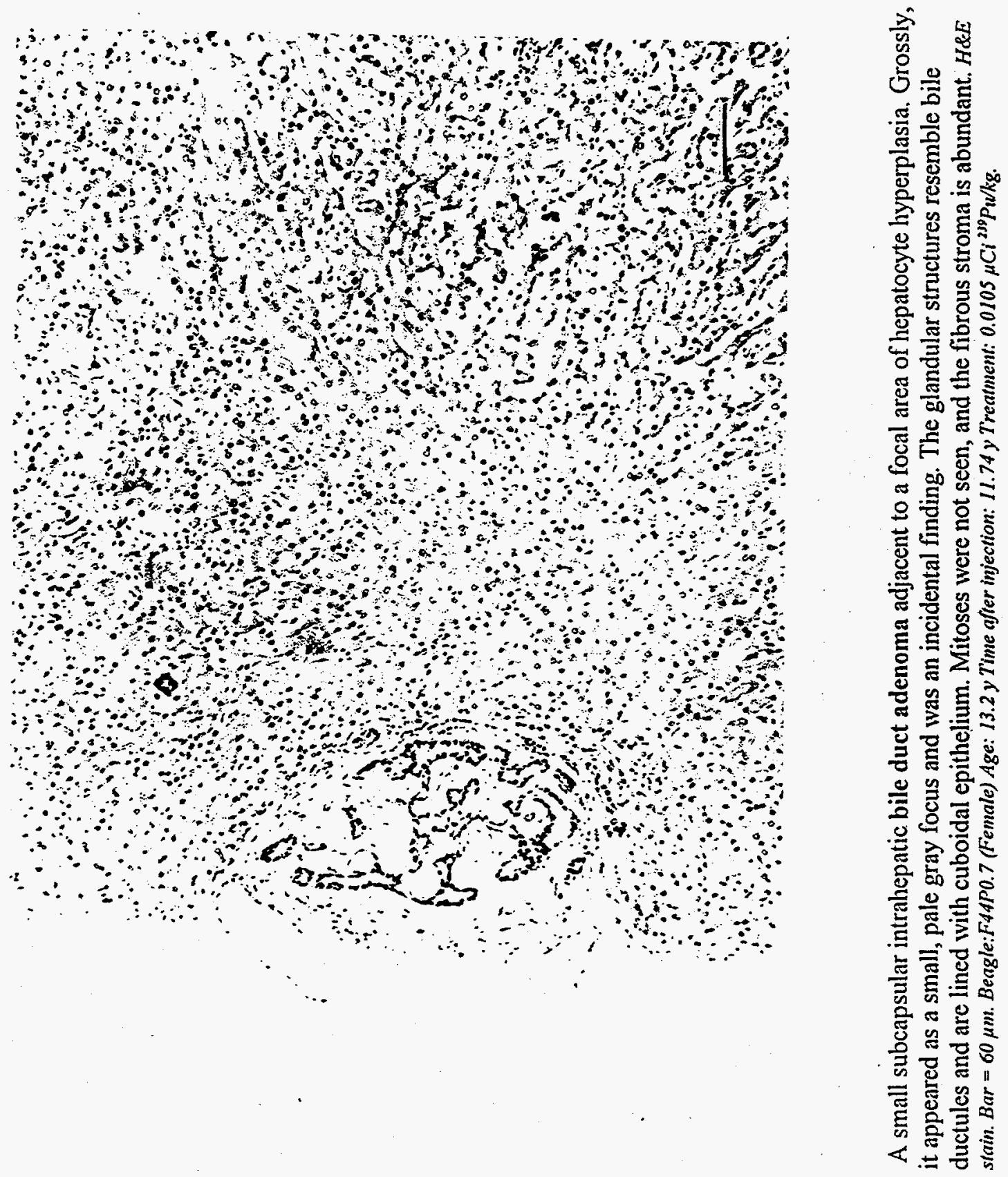

$\underbrace{\infty}_{0}$ 


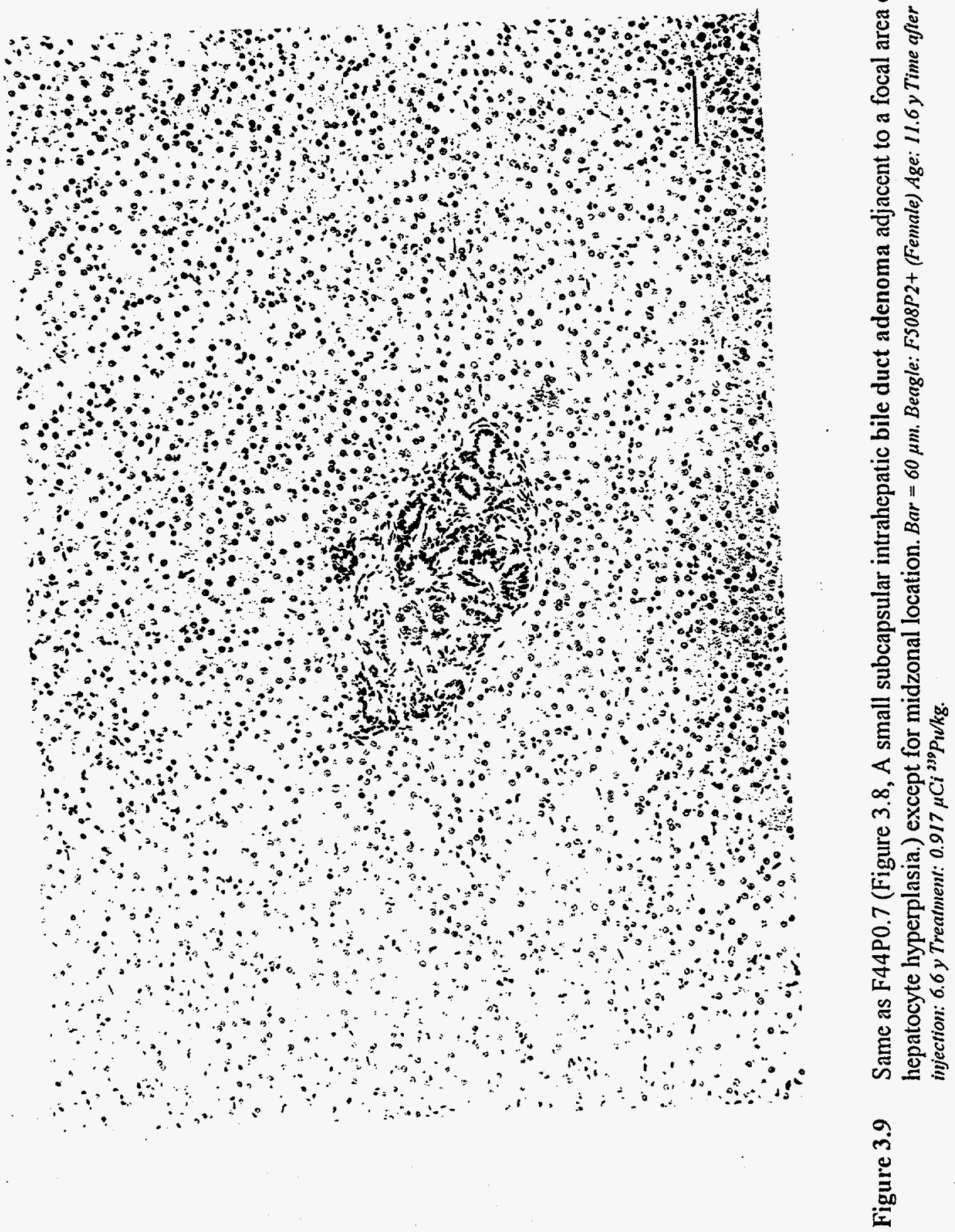




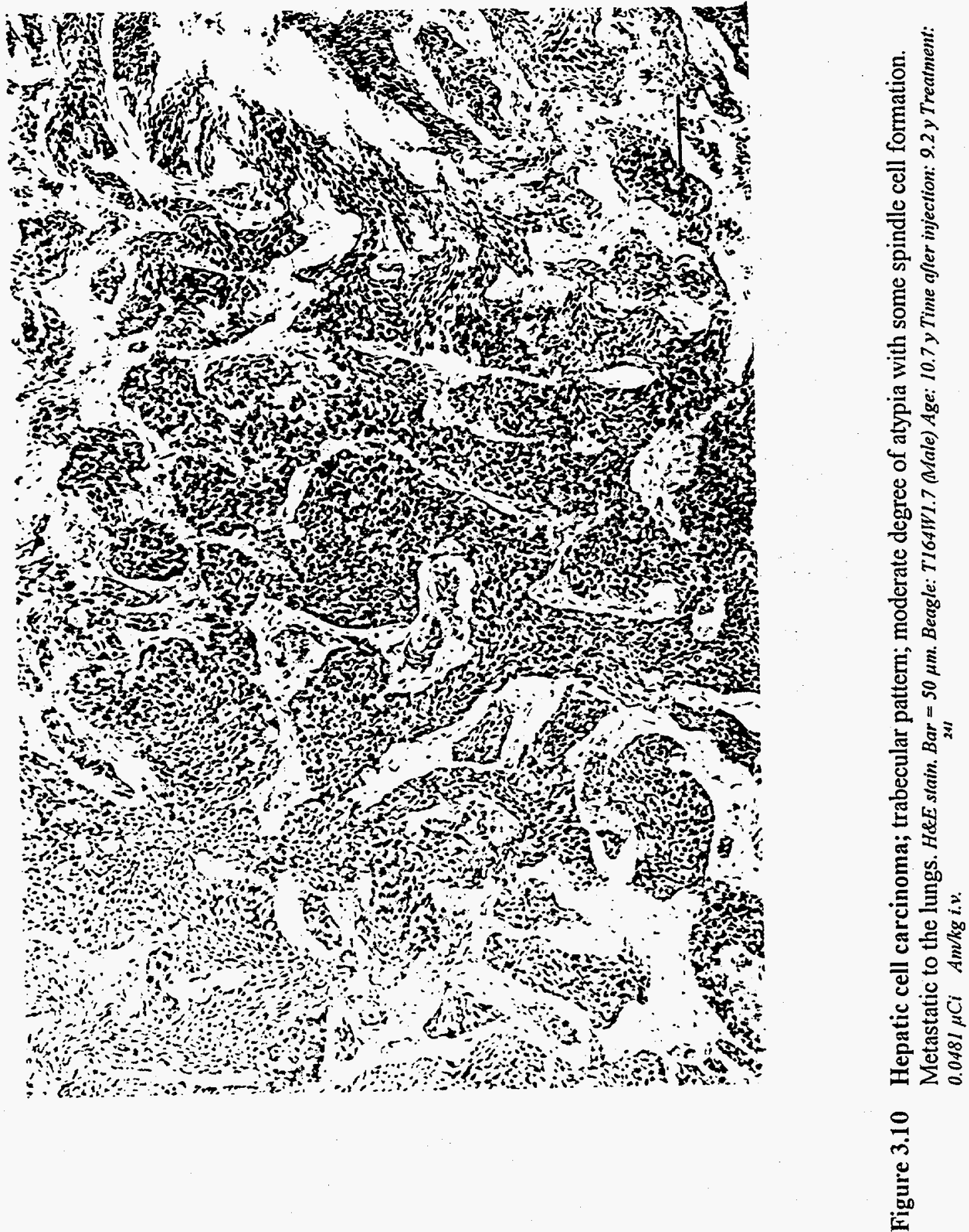




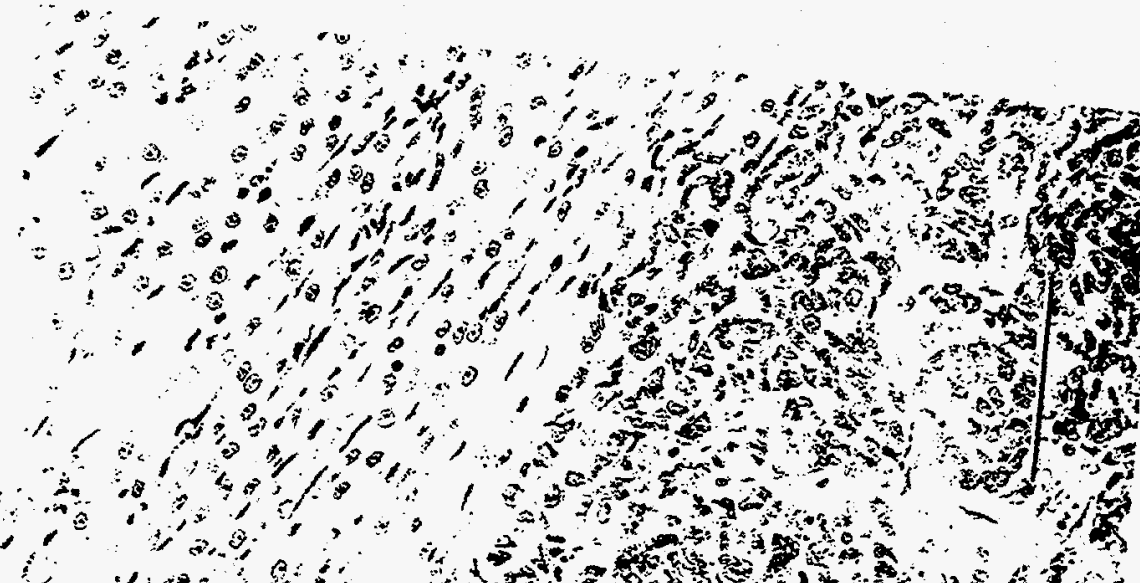

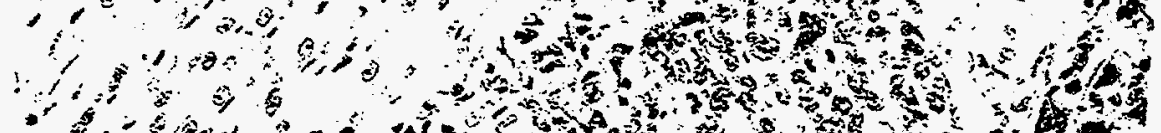

\%

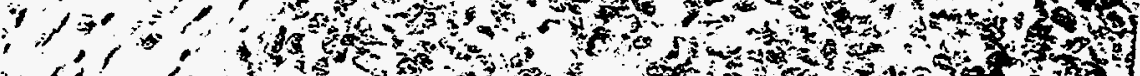

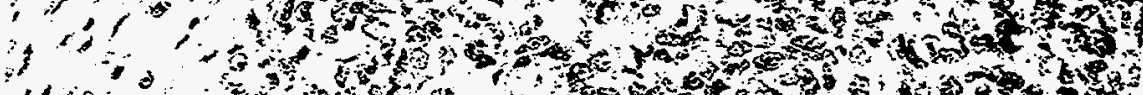

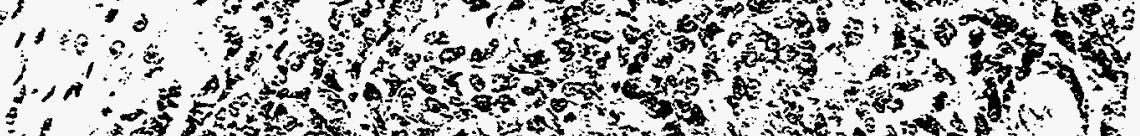

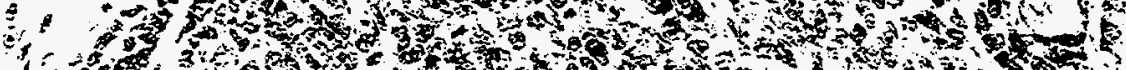

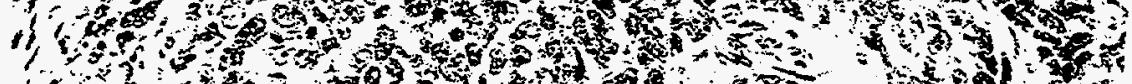

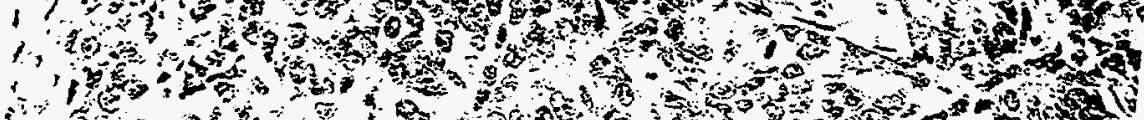

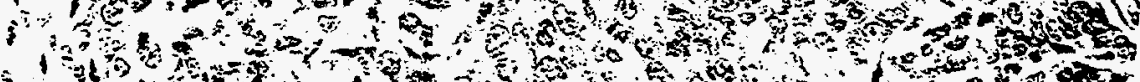

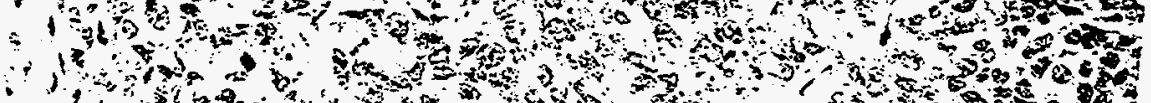

1, 1090 -

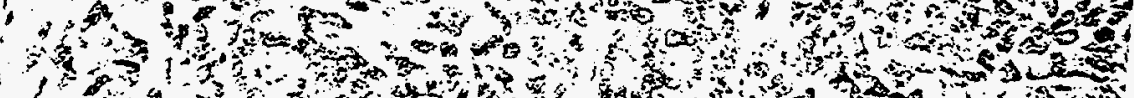

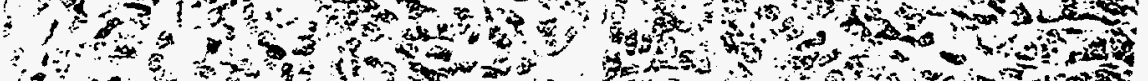

inction

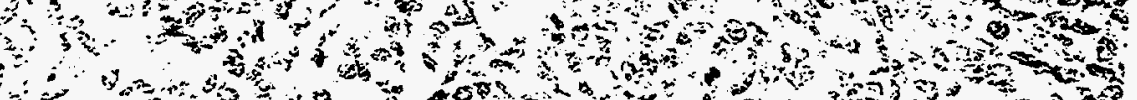

at

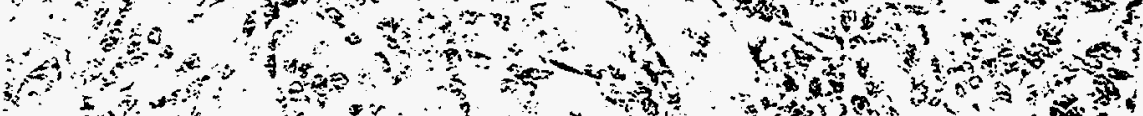

-

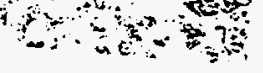




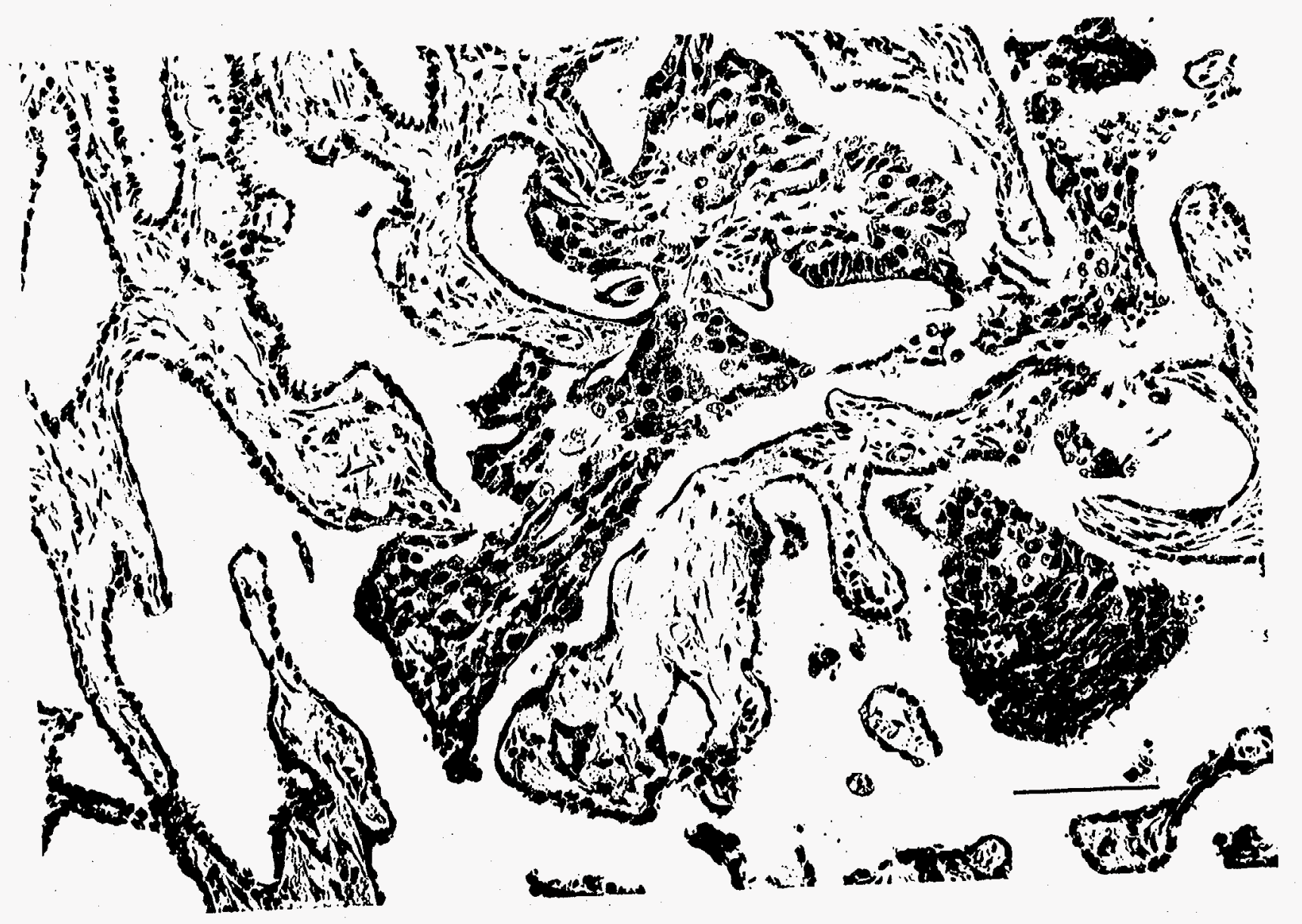

Figure 3.12 Intrahepatic cholangiocarcinoma; multiple cysts separated by a moderately dense stroma; epithelial pleomorphism evident in both the lining of the cystic cavities and the papillary projections; neoplastic epithelium varies from low cuboidal to tall columnar; complete loss of polarity in some regions. $H \& E$ stain. $B a r=60 \mu m$. Beagle: T164W1.7 Male) Age: 10.7 y Time after injection: 9.2 years Treatment: $0.0481 \mu \mathrm{Ci}{ }^{24} \mathrm{Am} / \mathrm{kg}$ i.v. 


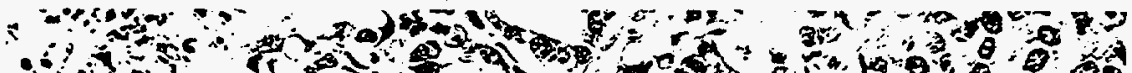

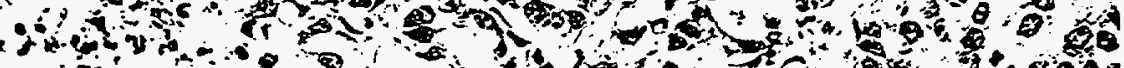

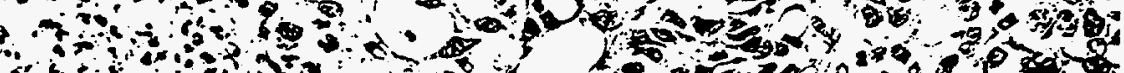

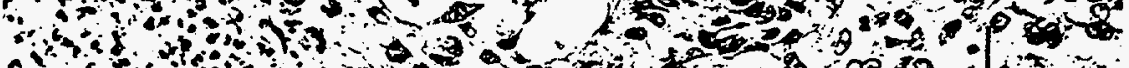

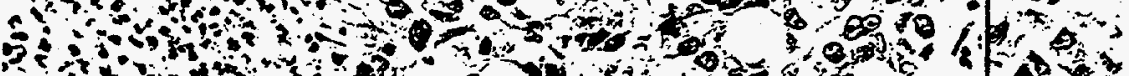

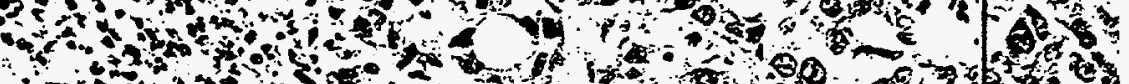
o en

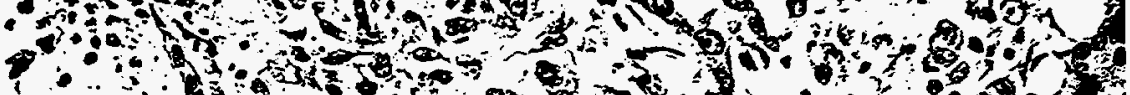

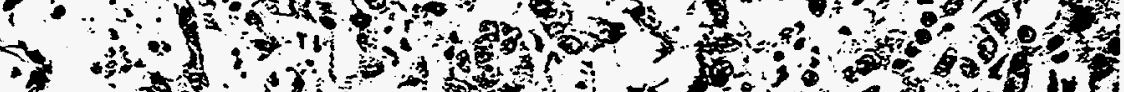

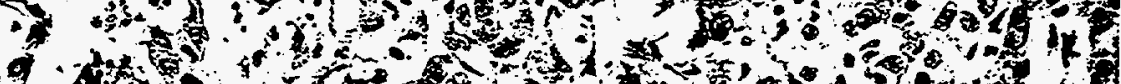
Afl

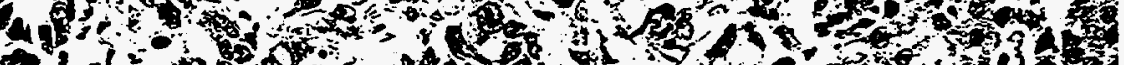

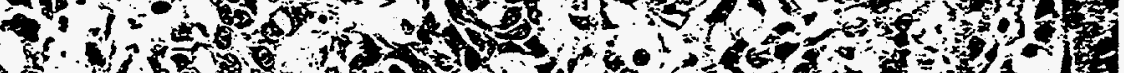

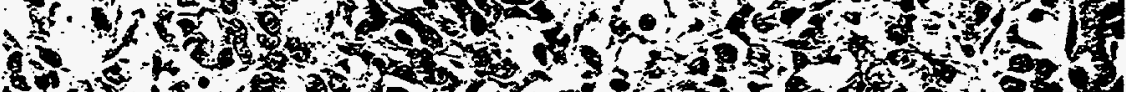

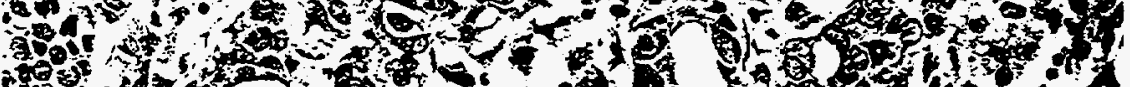

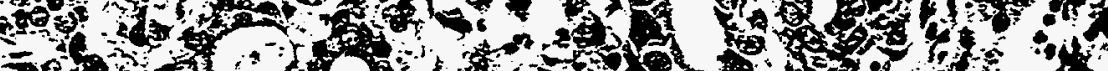

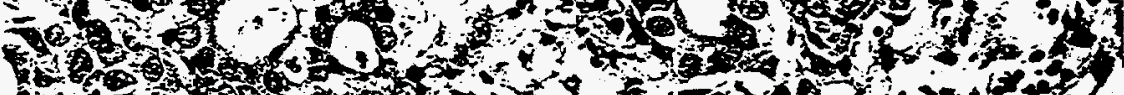

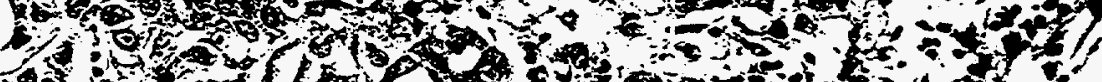

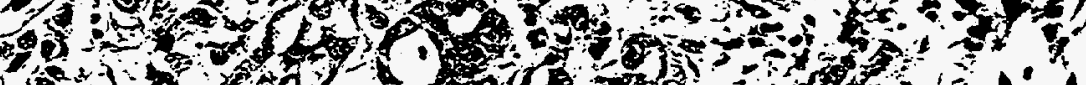
6. on

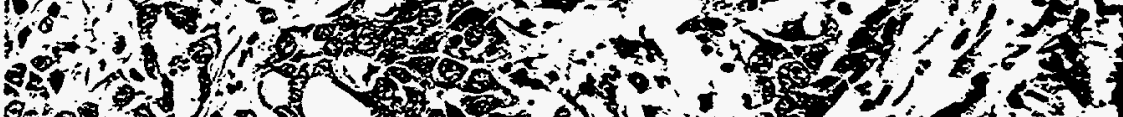
6. $-\omega^{2}$ 2

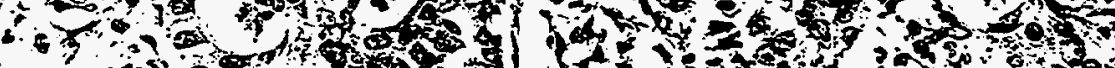
(5)

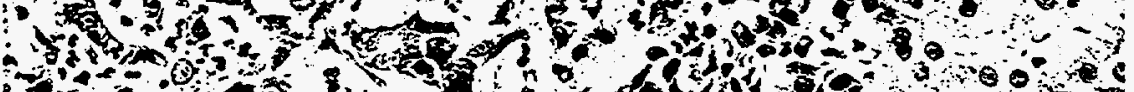

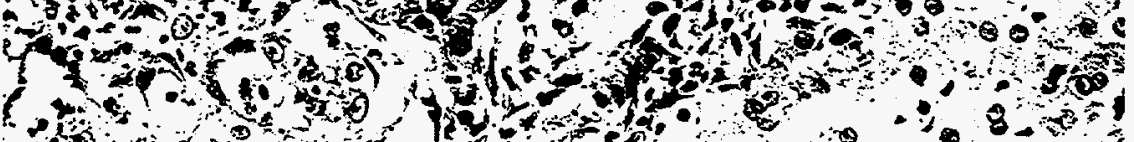

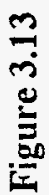




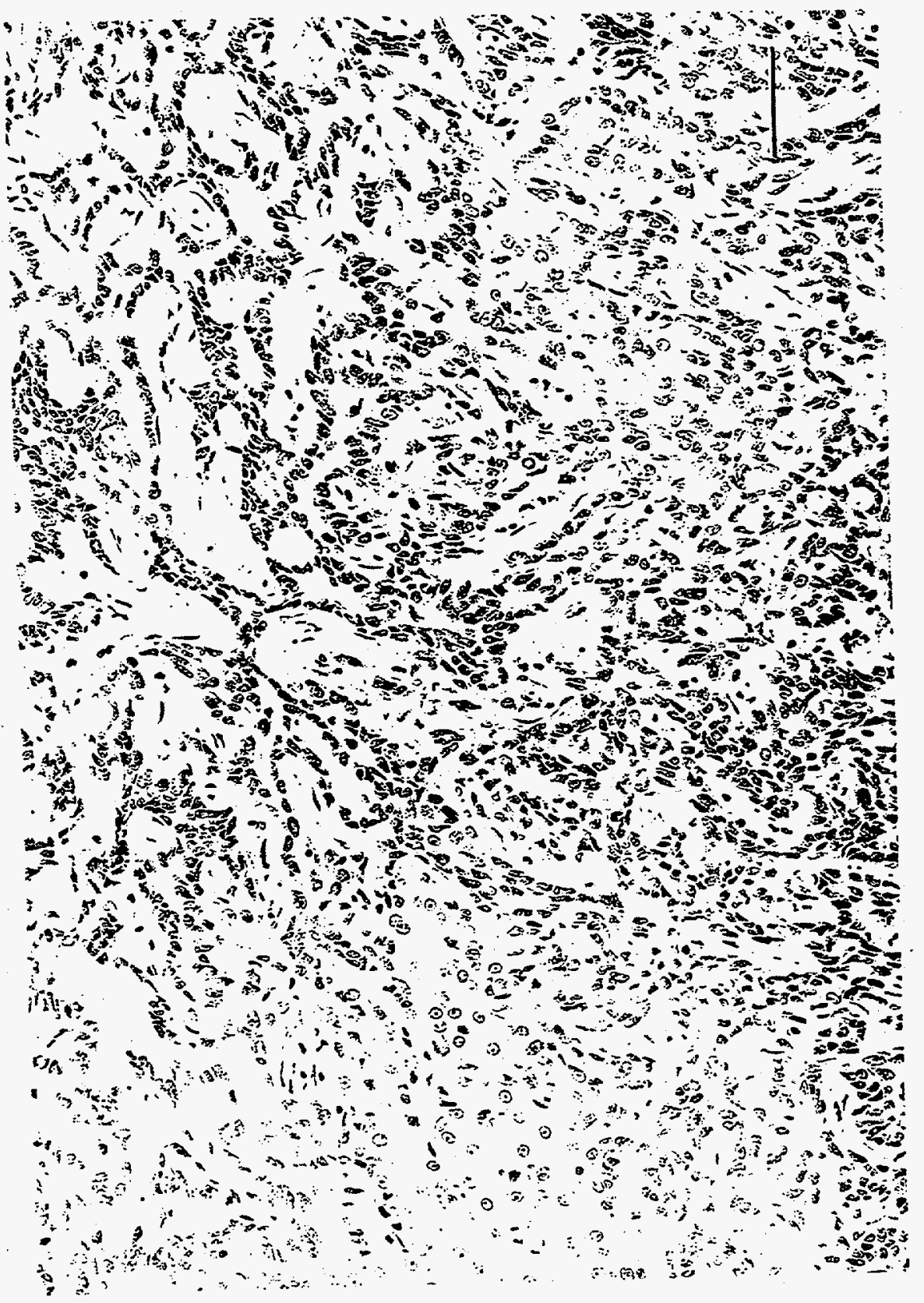

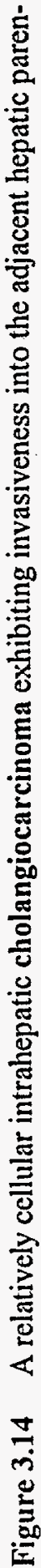

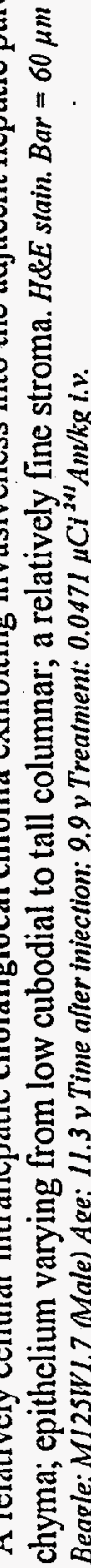


ard

Don a inom

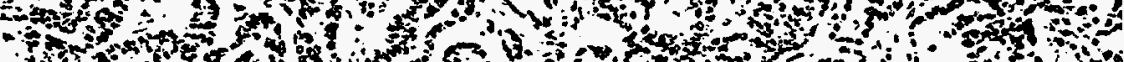
- 0 or ofon jon Hend as

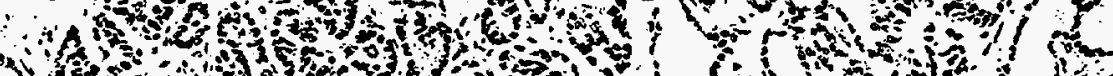
A (

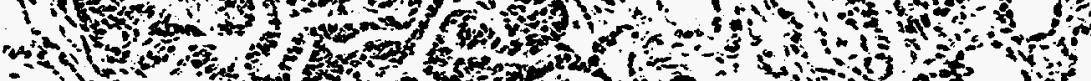
(n) -20

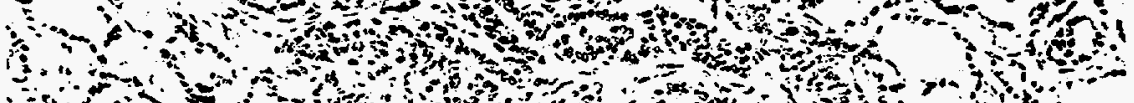

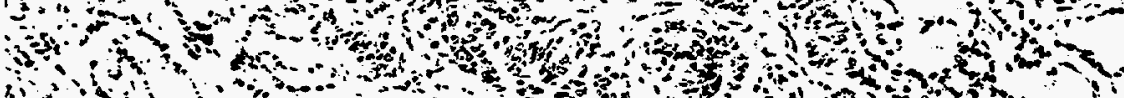
( ) $380^{\circ}=0$ ton 10 , Ho 0

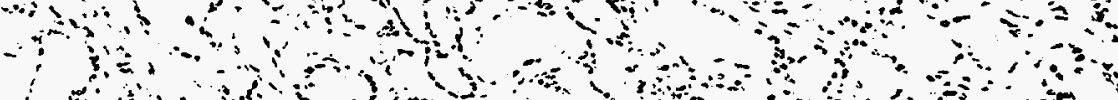

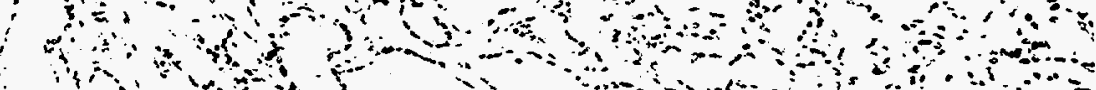

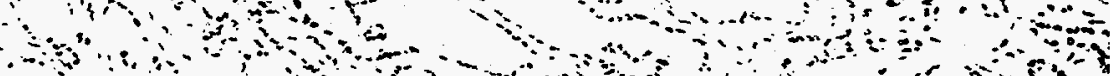
and a

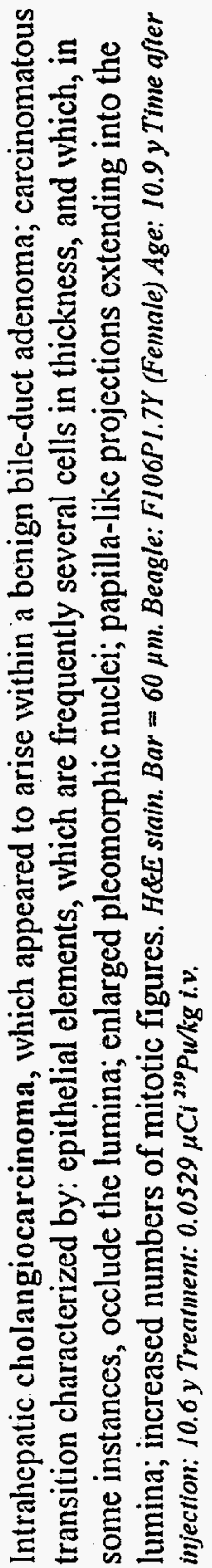

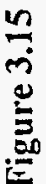




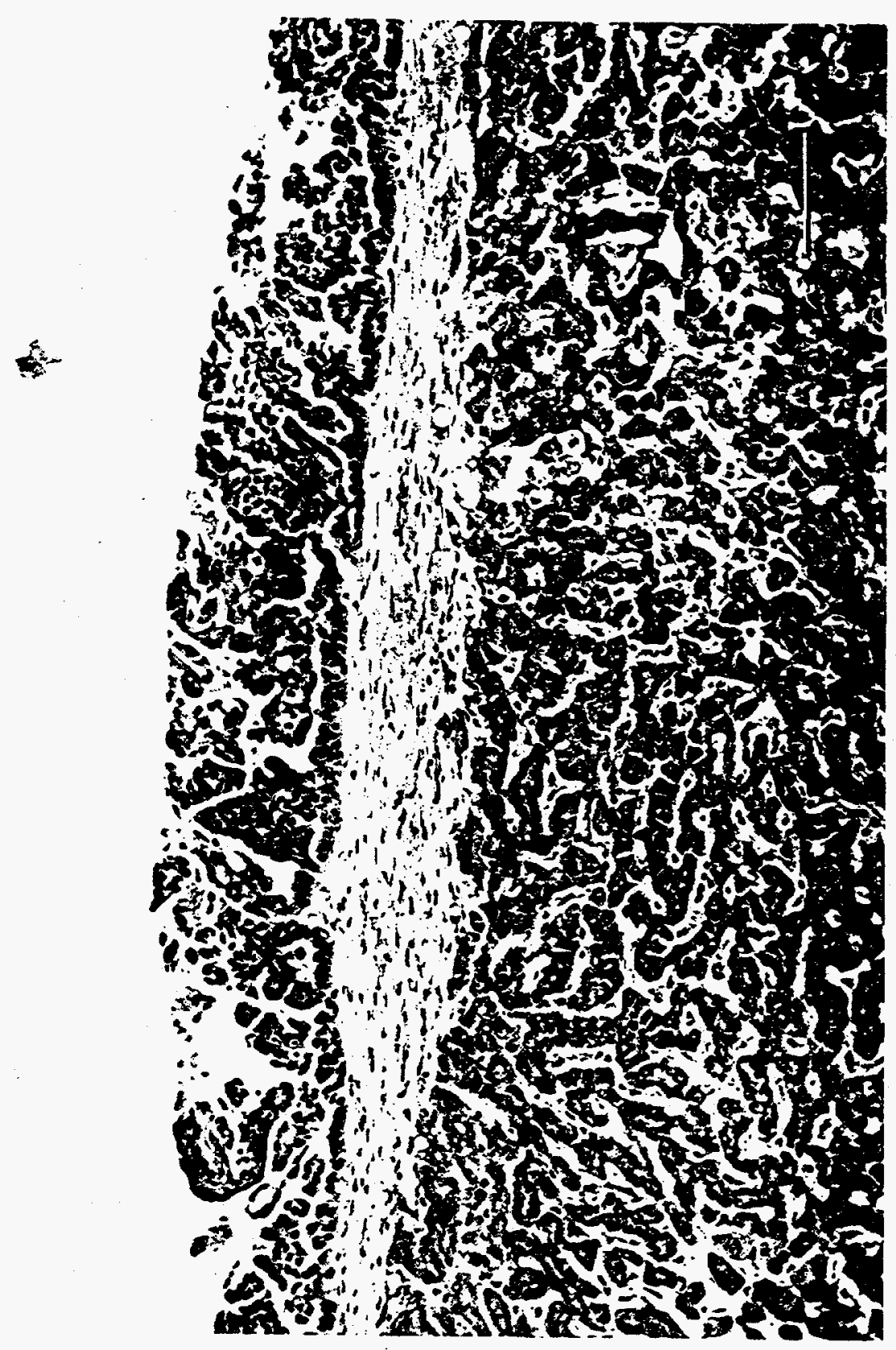

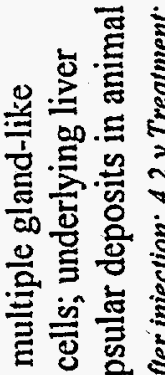

늉

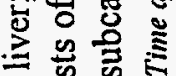

巳

등 可

品

氙芯总

\% 월

品

을

छ

은 믈

结

运

응. 를

可堅

营总至

言

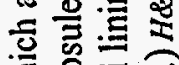

롱

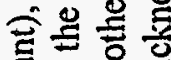

范资

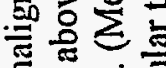

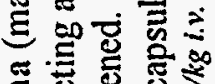

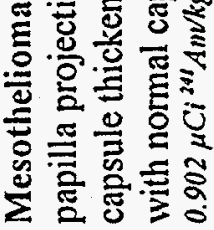

ำ 


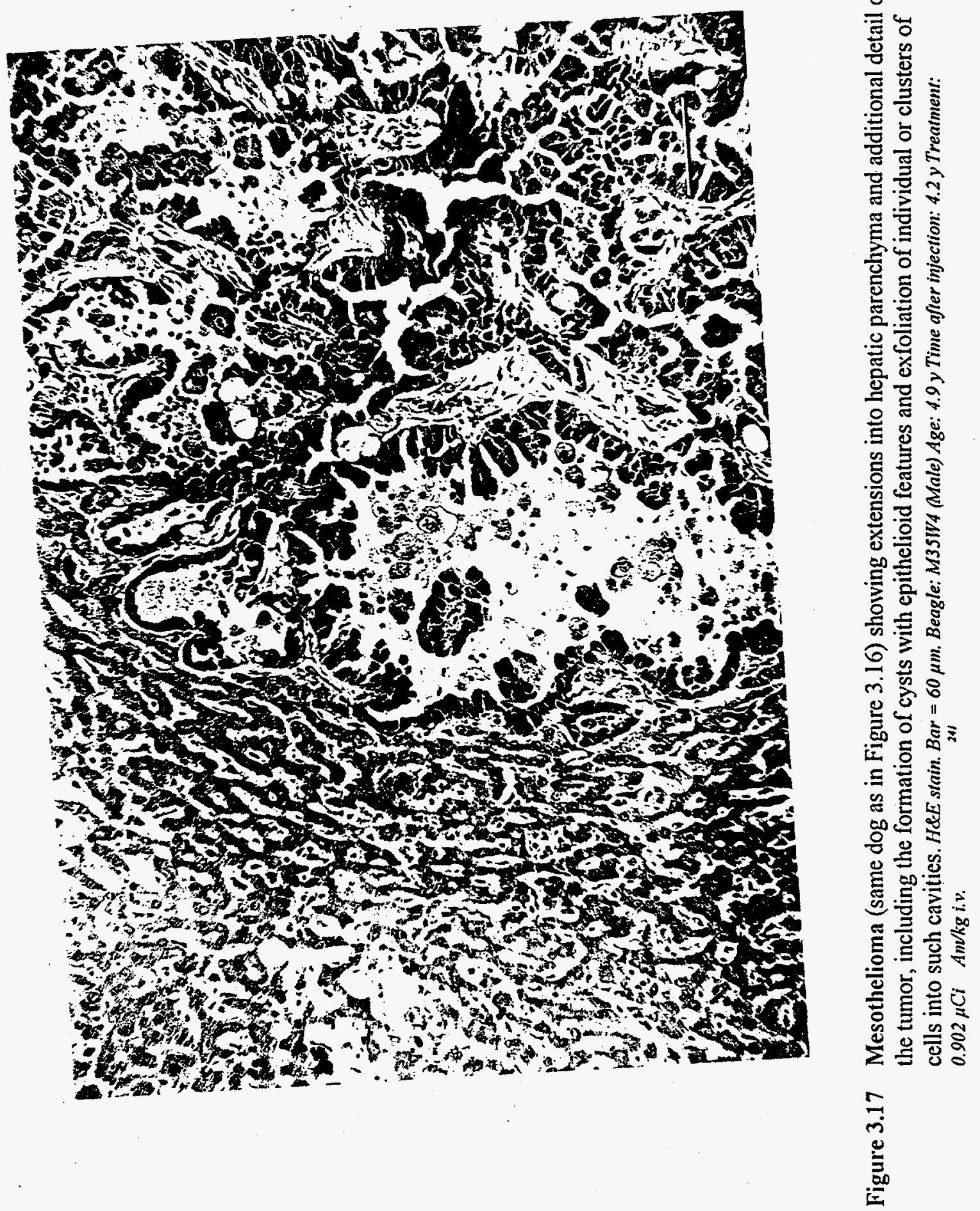



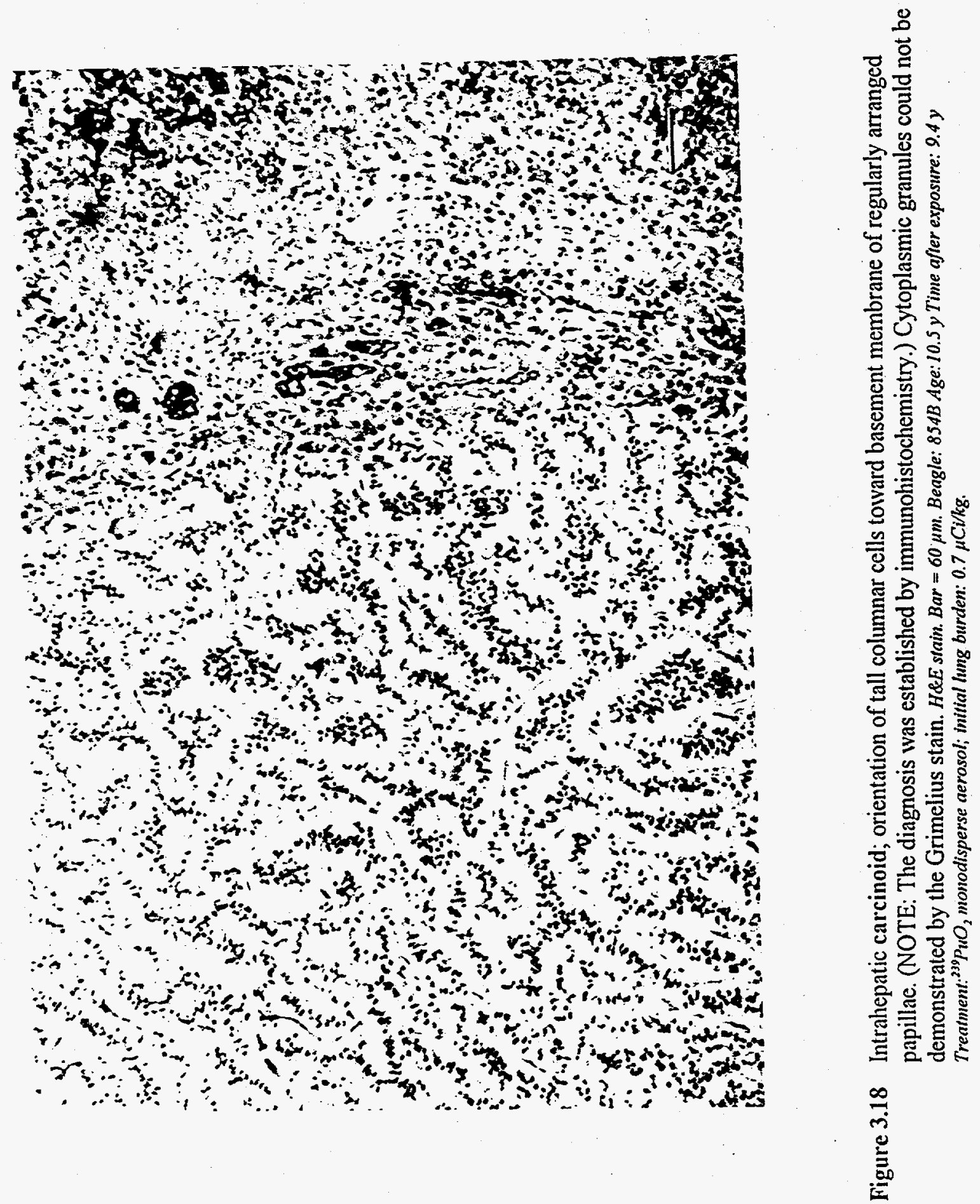

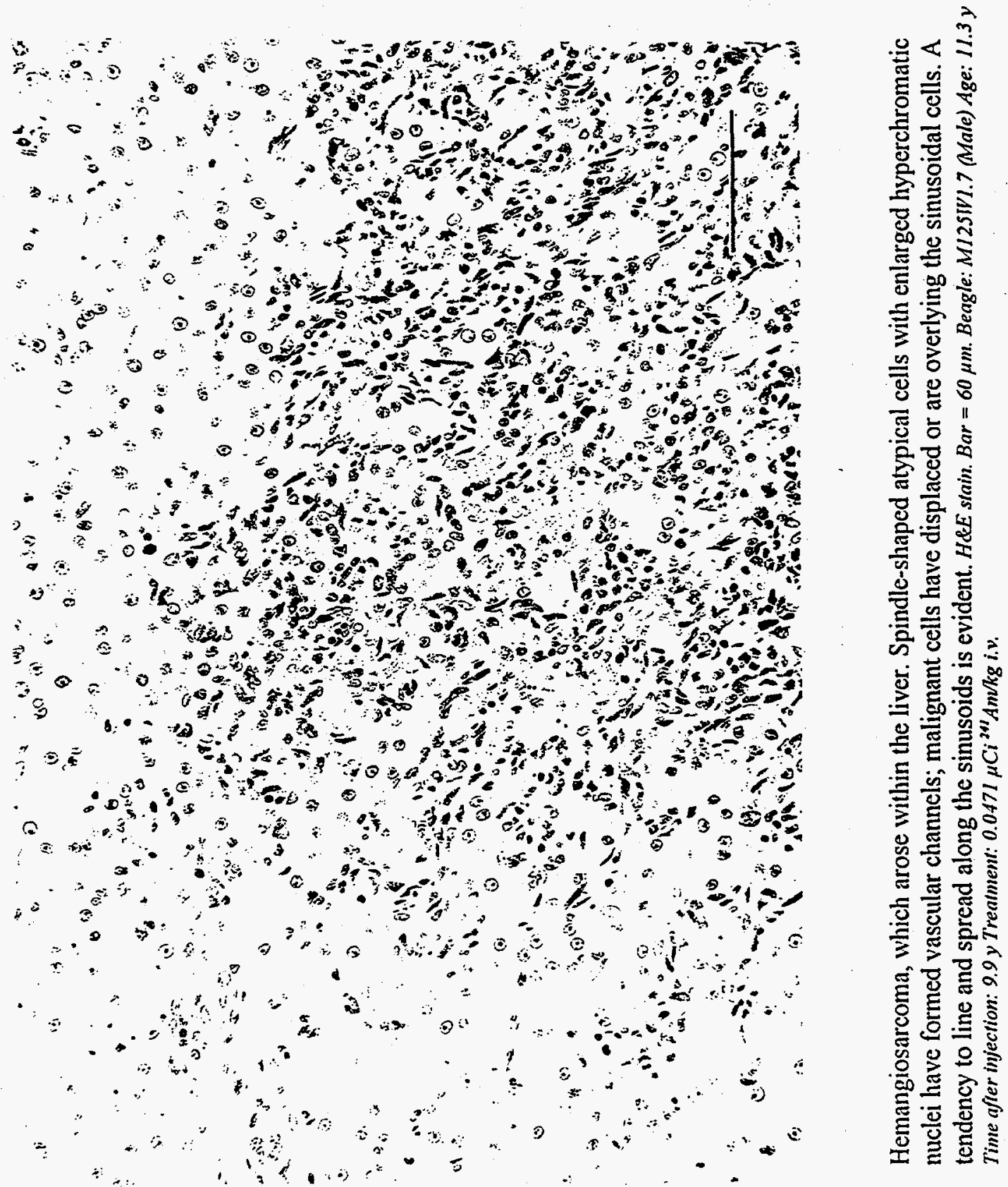

مَ 

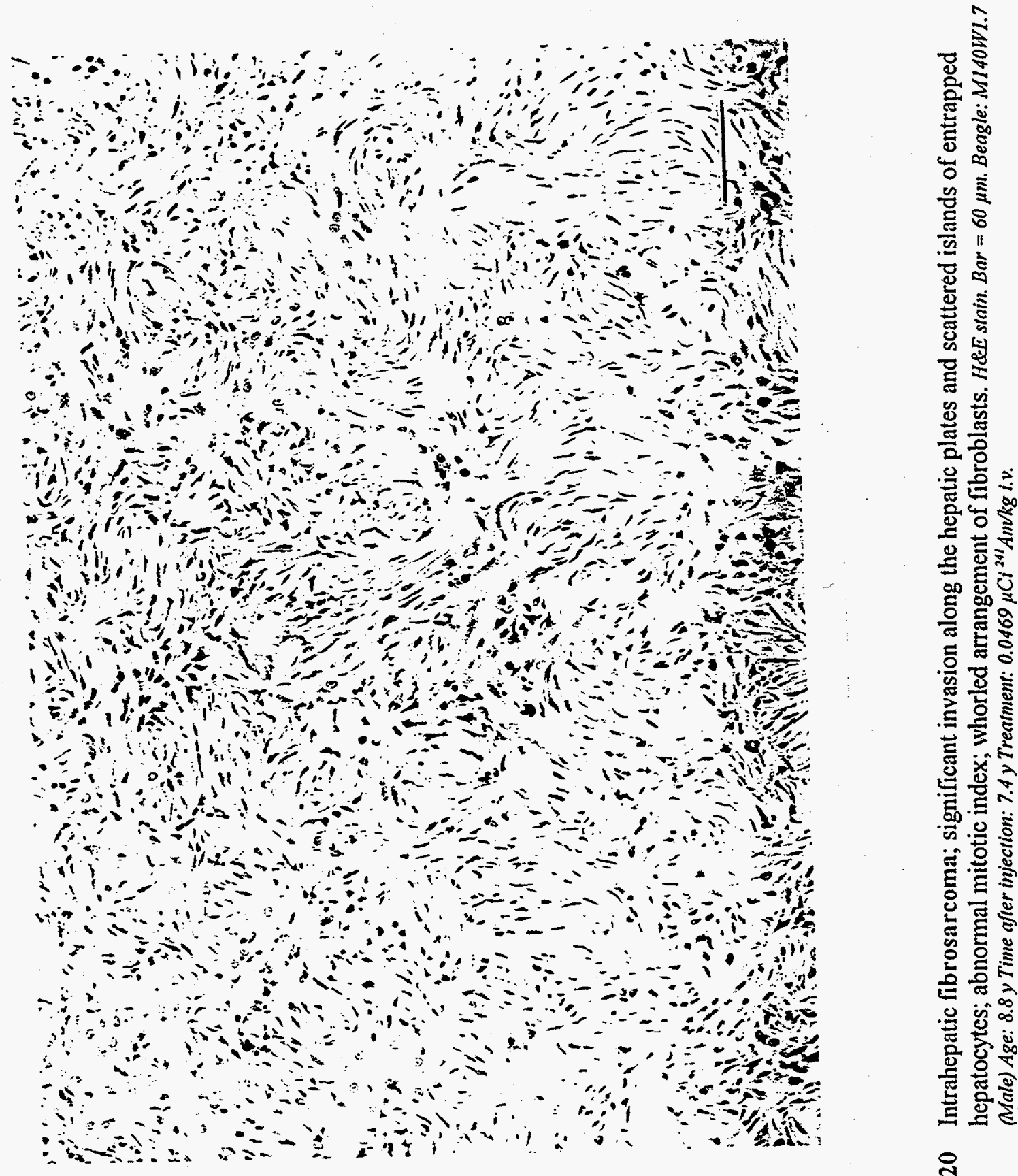

궁 

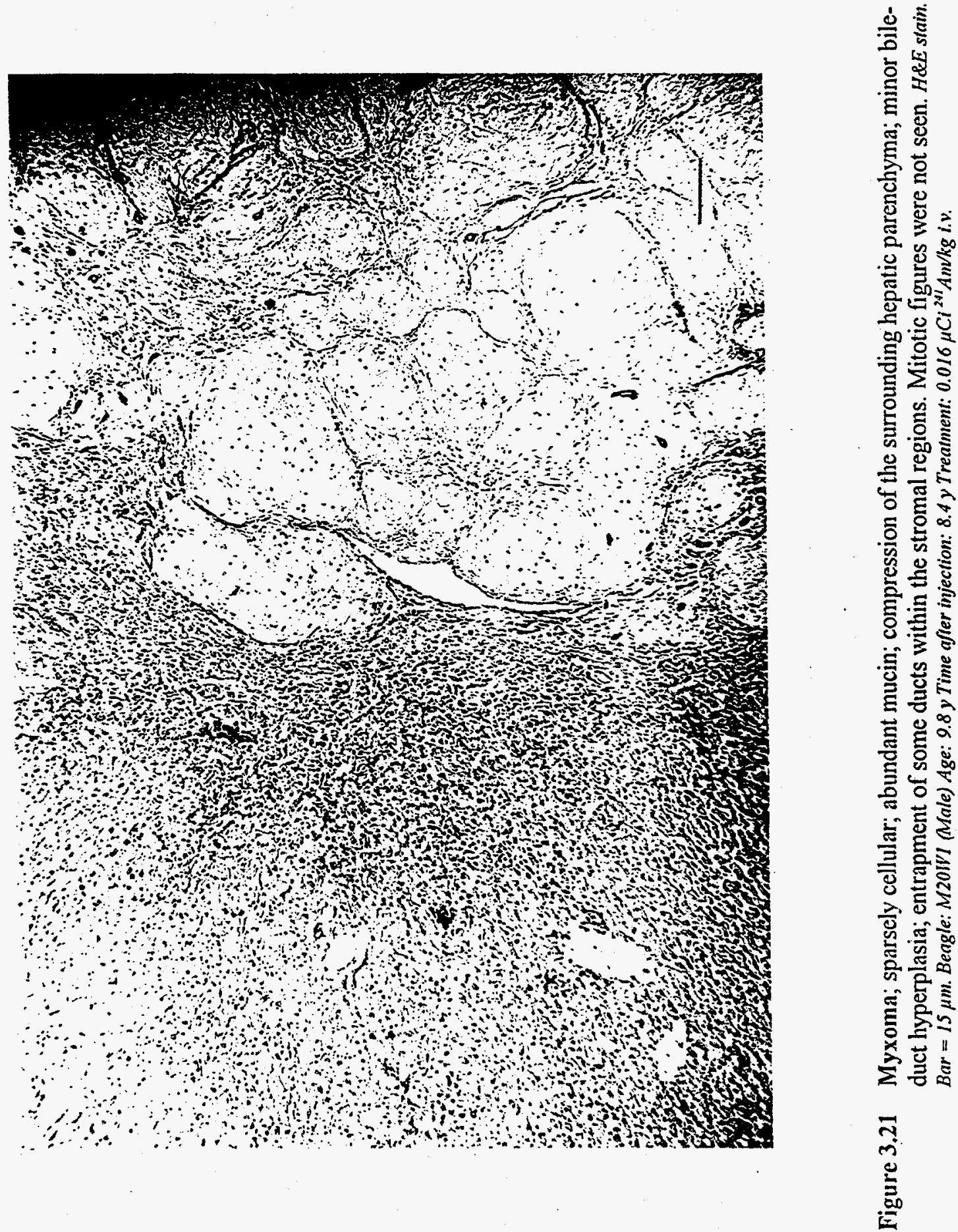


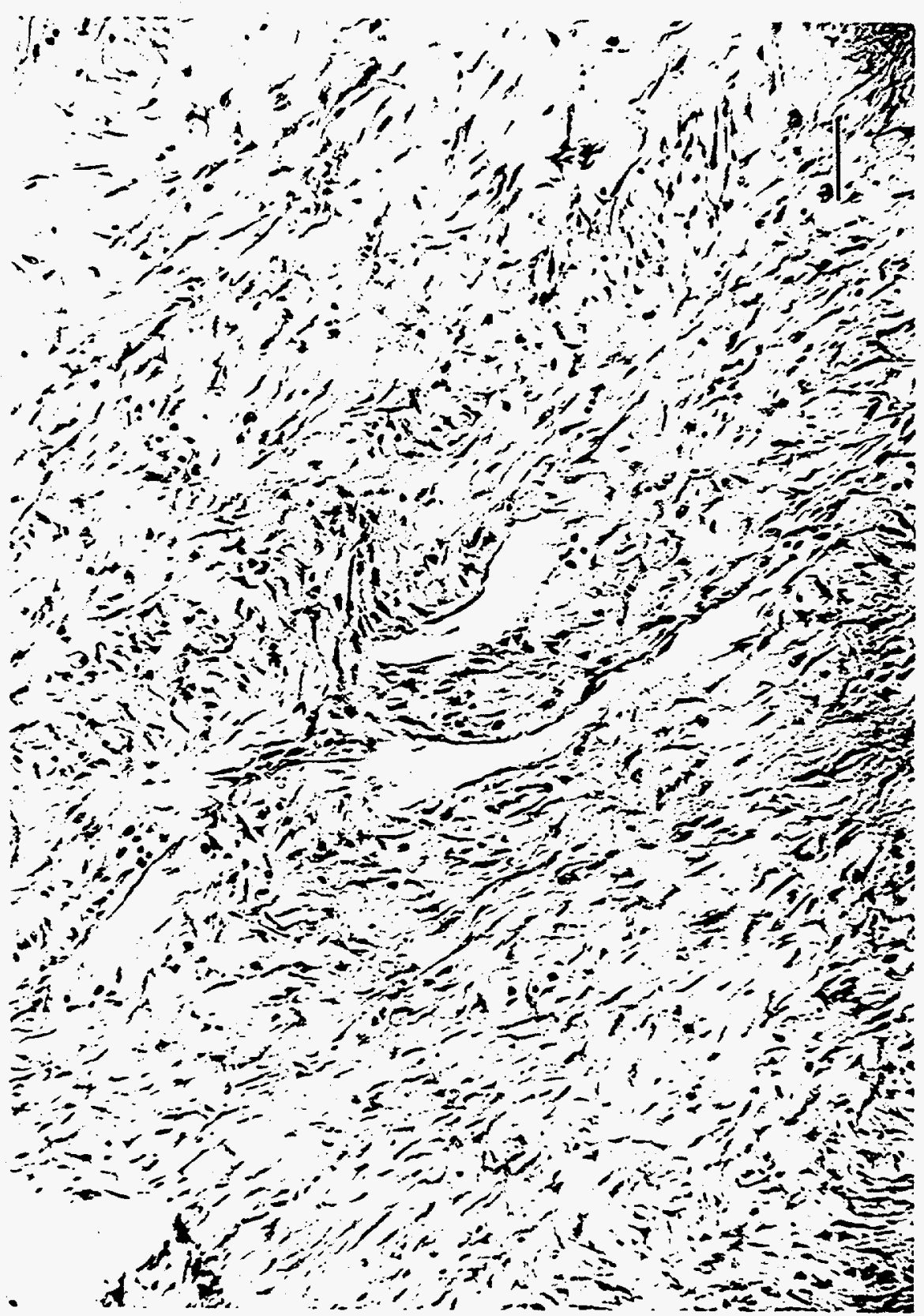

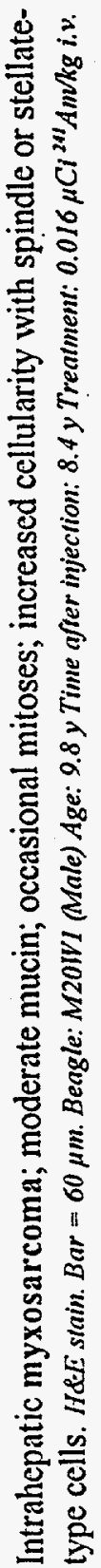

กั 


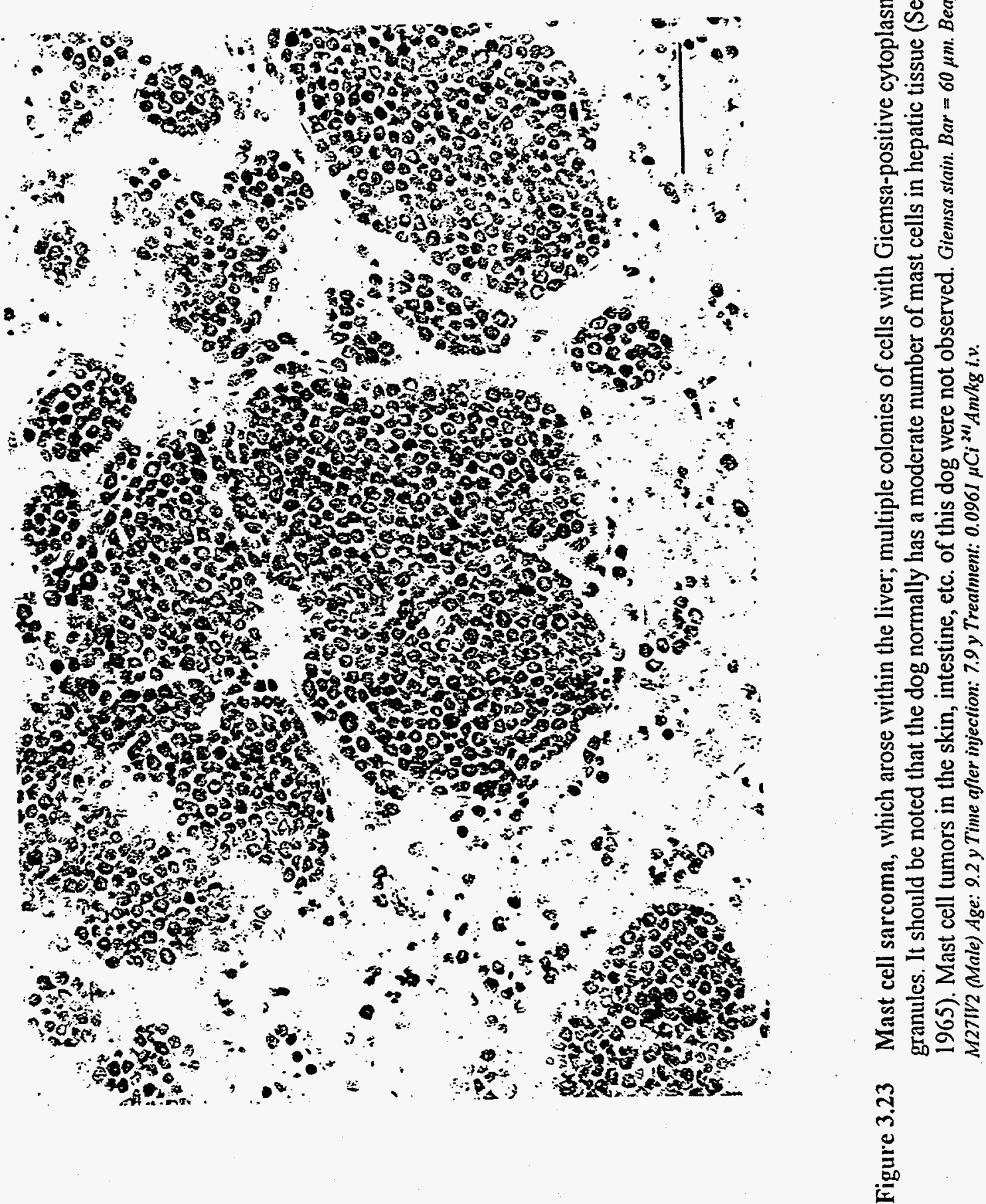




\title{
CHAPTER 4: Tumors of the Lung
}

\author{
Gerald E. Dagle \\ Pacific Northwest National Laboratory \\ Currently at Washington State University-Tri Cities \\ Fletcher F. Hahn \\ Inhalation Toxicology Research Institute
}

\section{INTRODUCTION}

The classification of lung tumors follows a modification of a nomenclature proposed by the World Health Organization (World Health Organization 1974) that is similar to other classification schemes (Moulton 1978; Moulton, von Tscharner, and Schneider 1981). The nomenclature emphasizes the histologic patterns rather than the site of origin of the tumors. Diagnoses were made from paraffin sections stained with hematoxylin and eosin but were augmented by special stains and transmission electron microscopy. It is anticipated that emerging techniques of molecular biology will help clarify the biological relevance of the histologic types (Gillett et al. 1992).

The principal source of material for this chapter came from studies of young beagle dogs given inhalation exposures to ${ }^{239} \mathrm{PuO}_{2}$ (at PNNL) or beta-emitting radionuclides in insoluble forms (at ITRI) for life-span dose effects observations (Park et al. 1986; McClellan et al. 1986). A unique feature of the studies done at PNNL was that mastectomies were performed on all the female dogs before exposure to prevent metastatic mammary tumors in the lung that could be confused with primary lung tumors. In this project, there were 66 lung tumors observed in 54 dogs $(51$ exposed dogs and 3 control dogs). For the purposes of recording the results, multiple tumors of the same histologic type found in different lobes were generally considered one primary tumor with intrapulmonary metastases. The classification system (Table 4.1) includes diagnoses made in other studies as well (Hahn et al. 1973; Hahn, Muggenburg, and Griffith 1992).

\section{DESCRIPTIONS}

\section{Epidermoid Carcinoma (Squamous Cell Carcinoma)}

The architecture is replaced by an irregular proliferation of stratified squamous epithelium, a few to several cells thick (Figure 4.1-4.2). The stratified squamous epithelium frequently lines large cystic cavities partially filled with necrotic debris. These cavities replace alveoli but leave intact the smaller bronchi; an apparent continuum of the stratified squamous epithelium and bronchial mucosa is frequently observed. The surfaces of the stratified squamous epithelium are generally nonkeratinized. Intercellular bridges and occasional keratin whorls are present. Ultrastructurally, tonofilaments and desmosomes are prominent. Invasion of blood vessels occasionally occurs. Metastases sometimes occur to the tracheobronchial lymph nodes and systemic organs. 


\section{Lung}

Table 4.1. Lung Neoplasia Nomenclature, Index of Figures, Snodog Database Codes, and Incidence.

\begin{tabular}{|c|c|c|c|c|}
\hline \multirow[b]{2}{*}{ Diagnosis } & \multirow[b]{2}{*}{ Figure } & \multirow{2}{*}{$\begin{array}{l}\text { SNODOG } \\
\text { Morphology } \\
\text { Code }\end{array}$} & \multicolumn{2}{|c|}{$\begin{array}{c}\text { Percent } \\
\text { Incidence }\end{array}$} \\
\hline & & & $P^{b}$ & $U^{e}$ \\
\hline Epidermoid carcinoma & $4.1-2$ & M807030 & 5 & \\
\hline \multicolumn{5}{|l|}{ Adenorcarcinoma } \\
\hline Bronchioloalveolar carcinoma & $4.3-4$ & $\mathrm{M} 825030$ & 39 & 19 \\
\hline Papillary adenocarcinoma & 4.5 & M826030 & 32 & 66 \\
\hline Solid carcinoma & 4.6 & M823030 & 1 & 0 \\
\hline Adenosquamous carcinoma & 4.7 & M856030 & 23 & 9 \\
\hline Anaplastic carcinoma & & M802030 & 0 & 0 \\
\hline Small cell & & M800230 & & \\
\hline Large cell & 4.8 & $\mathrm{M} 801230$ & & \\
\hline Sarcoma & & & 0 & \\
\hline Hemangiosarcoma & 4.9 & M912030 & 0 & 30 \\
\hline Fibrosarcoma & 4.10 & M881030 & & \\
\hline Chondrosarcoma & 4.11 & M922030 & & \\
\hline Leiomyosarcoma & 4.12 & M889030 & & \\
\hline Malignant fibrous histiocytoma & 4.13 & M883030: & & \\
\hline Osteosarcoma & 4.14 & M918030 & & \\
\hline Mixed tumor, malignant & 4.15 & M894030 & 0 & 0 \\
\hline Carcinoid tumor, malignant & 4.16 & M824030 & 0 & 0 \\
\hline Benign lung tumors & & & 0 & \\
\hline Adenoma & 4.17 & M814000 & 0 & 0 \\
\hline Papillary adenoma & 4.18 & M826000: & & \\
\hline Fibroma & 4.19 & M881000 & & \\
\hline Carcinoid tumor, benign & & M824000 & & \\
\hline Mixed tumor, benign & 4.20 & M808140 & & \\
\hline Bronchial gland & & & 0 & 3 \\
\hline Adenoma & 4.21 & M81400B & & \\
\hline Adenocarcinoma & & M81403B & & \\
\hline Mesothelioma & & s. & 0 & \\
\hline Benign & & M905000 & 0 & 0 \\
\hline Malignant & 4.22 & M905030 & & \\
\hline \multicolumn{5}{|l|}{ Non-neoplastic lesions } \\
\hline Adenomatous hyperplasia & & M724901 & & \\
\hline Squamous metaplasia & & M724903 & & \\
\hline Radiation pneumonitis & 4.23 & M497701 & & \\
\hline
\end{tabular}




\section{Adenocarcinoma}

\section{Bronchioloalveolar Carcinoma}

Bronchioloalveolar carcinomas consist of a proliferation of cuboidal to low-columnar, nonciliated epithelium along alveolar septa (Figure 4.3). The cells tend to pile up on the surface and may slough into the lumen of alveoli. In larger masses there is some tendency toward papillary proliferation. The epithelial cells have round to oval, nuclei, with evenly distributed chromatin and indistinct nucleoli. The cytoplasm is homogenous, negative for mucus stains, and may have tapered apical cytoplasm. The stroma is scant but has a tendency for foci of osseous metaplastia (Figure 4.4). There is a tendency for fronds of epithelial cells to extend into adjacent alveoli. Lymphatic invasion may be very prominent in larger masses. Invasion of blood vessels and nerves is unusual. Multiple masses are often present in several lobes. For purposes of coding, the lobe with the largest mass is considered the primary site, and smaller masses are considered metastatic sites.

\section{Papillary Adenocarcinoma}

The predominant feature is the papillary proliferation of nonciliated columnar epithelial cells on a fibrovascular stroma that may be fine or coarse (Figure 4.5). The neoplastic cells compress or replace normal architecture of the lung. The lining epithelial cells are usually columnar but may be cuboidal, and form a single layer of cells with basilar nuclei. The individual nuclei may have clumped chromatic and prominent nucleoli. The epithelial cells seldom stain for mucus but there is frequently mucus-positive material in the lumen. The stroma frequently has prominent spicules of metaplastic bone. Lymphatic invasion and metastases are frequent, as with bronchioloalveolar carcinomas.

\section{Solid Carcinoma}

The general appearance is solid sheets and chords of anaplastic epithelial cells replacing normal architecture of the lung (Figure 4.6). The individual cells generally have scant cytoplasm and large nuclei with prominent nucleoli.

\section{Adenosquamous Carcinoma (Combined Carcinoma)}

These tumors contain mixtures of morphologic patterns of adenocarcinomas and epidermoid carcinomas (Figure 4.7). The papillary pattern is the usual morphologic form of adenocarcinoma present. Prominent portions of both patterns need to be present, at least one fourth of one morphologic pattern or the other. The glandular proliferation tends to form tubules or ducts with prominent stroma and to form a continuum with nonstratified squamous epithelium.

\section{Anaplastic Carcinoma}

\section{Small-Cell Carcinoma}

None of these tumors has been reported in the beagle colonies at ANL; University of California, Davis; ITRI; PNNL; or University of Utah.

\section{Large-Cell Carcinoma}

The tumor is characterized by large anaplastic epithelial cells that have abundant eosinophilic cytoplasm and large nuclei, with prominent large nucleoli (Figure 4.8). Multiple nuclei may be present. The individual cells or clumps of cells fill the alveoli but do not invade large interstitial structures of the lung. The anaplastic cells appear to arise from alveolar epithelial lining cells. 
Sarcoma

\section{Hemangiosarcoma}

These sarcomas of vascular endothelial origin arise from the alveolar regions and have cavernous or capillary patterns (Figure 4.9). They consist of blood-filled cavities lined with plump anaplastic endothelial cells, having large oval nuclei with prominent nucleoli. The cytoplasm is flattened and homogenous. Hemorrhage frequently extends into alveoli and tissues surrounding the neoplasms. Widespread metastases frequently occur.

\section{Fibrosarcoma}

These tumors consist of spindle-shaped cells with anaplastic nuclei and resemble fibrosarcomas in other organs of the body (Figure 4.10). In the lungs of irradiated dogs these tumors originate in alveolar portions of the lungs. Metastases may occur.

\section{Chondrosarcoma}

The tumor consists of confluent lobules of hyaline cartilage (Figure 4.11). The lobules, which are not supplied with blood vessels, have degeneration in the center. The lobules appear to be expanding with an increased density of mildly anaplastic chondrocytes toward the periphery of the lobules.

\section{Leiomyosarcoma}

The tumor consists of an irregular proliferation of anaplastic smooth-muscle cells originating in bronchial walls (Figure 4.12).

\section{Malignant Fibrous Histiocytoma}

This is a solitary tumor that arises in the hilar region and may invade ainways and arteries (Figure 4.13). Metastasis, however, is to local lymph nodes. The tumor is composed of a mixture of histiocytes and fibroblasts. The fibrous tissue has a swirled and, occasionally, a storiform pattern. The histiocytic cells may be very pleomorphic, and giant, multinucleated cells may be present. In only one case has this tumor been found as a primary lung tumor.

\section{Osteosarcoma}

In the lungs of irradiated dogs, these tumors are composed of hyperchromatic pyriform cells (osteoblasts) that produce varying amounts of connective tissue and immature bone. Frequently, mineralized bone spicules are present. In these histologic features, the osteosarcomas of the lung resemble such tumors elsewhere in the body. Most of these osteosarcomas appear to arise from osseous metaplasia in the stroma of lung carcinomas (Figure 4.14).

\section{Mixed Tumor, Malignant}

These are large confluent masses of proliferating fibroblasts mixed with ducts or glands of anaplastic epithelial cells (Figure 4.15).

\section{Carcinoid Tumors}

These tumors are rare in the dog and have not been reported in the beagle dog colonies at.ANL; University of California, Davis; PNNL; or University of Utah. An atypical neuroendocrine carcinoma has been reported (Harkema et al. 1992) in an unexposed dog in the ITRI (Figure 4.16). This tumor was a nonencapsulated solid carcinoma composed of lobules of closely packed, round-to-oval cells separated by a fine stroma. The cells had abundant eosinophilic or clear cytoplasm that contained dense core granules and had neuron-specific enolase staining. 
Benign Lung Tumors

Adenoma

A focal proliferation of cuboidal epithelial cells that conform to an alveolar (Figure 4.17) or papillary (Figure 4.18) pattern. The individual cells are uniform and regular and form a single lining layer. The cell nuclei are uniform, without prominent nucleoli. The surrounding alveolar parenchyma is compressed.

Fibroma

A focal proliferation of fibroblasts producing abundant fibrous tissue (Figure 4.19). They usually occur in the parenchyma of the lung.

\section{Carcinoid Tumor, Benign}

None of these tumors has been reported in the beagle colonies at ANL; University of California, Davis; ITRI; PNNL; or University of Utah.

Mixed Tumor, Benign 4.20).

A glandular and fibrous proliferation not infiltrating adjacent pulmonary parenchyma (Figure

\section{Bronchial Gland Tumors}

\section{Adenoma}

A focal proliferation of mucus-producing cells supported by a thick, fibrous stroma. These tumors are associated with the larger ainways.

\section{Adenocarcinoma}

An infrequent tumor that has a close association with bronchi. These tumors form glandular structures lined by a high-columnar epithelium (Figure 4.21).

\section{Mesothelioma}

Mesotheliomas arise from the mesothelium without apparent involvement of pulmonary parenchyma. They consist of a papillary proliferation of anaplastic cells without associated fibrous stromas (Figure 4.22).

\section{Adenomatous Hyperplasia}

Adenomatous hyperplasia consists of the focal proliferation of alveolar or bronchiolar epithelial cells that line alveolar septa with cuboidal or low-columnar epithelium. The alveolar architecture is retained, and the epithelial proliferation is not clearly associated with inflammatory reactions. This contrasts with the more diffuse, reactive, alveolar epithelial hyperplasia associated with radiation pneumonitis and with bronchioloalveolar neoplasms, where the normal architecture of the lung is replaced.

\section{Squamous Metaplasia}

Squamous metaplasia can occur in alveolar regions of the lung without any clear association with bronchioles or bronchi. It is frequently associated with pulmonary fibrosis.

\section{Radiation Pneumonitis}

Acute radiation pneumonitis due to inhalation of radionuclides is characterized by congestion, alveolar inflammatory exudates, interstitial cellular infiltrates, desquamation of bronchiolar and 
alveolar epithelium with epithelial regression, vascular injury and repair, and pulmonary fibrosis (Slauson et al. 1976, Slauson, Hahn, and Chiffelle 1977). Conspicuous vascular and interstitial inflammatory reactions are much more prominent in animals exposed to beta-emitting radionuclides.

Chronic radiation pneumonitis due to the inhalation of radionuclides is characterized by interstitial fibrosis associated with alveolar epithelial cell hyperplasia and increased numbers of alveolar macrophages (Figure 4.23). Interstitial cellular infiltrates and vascular obliterative lesions may also be present. The interstitial fibrosis has a multifocal distribution primarily associated with subpleural and peribronchial areas of all lobes. Autoradiographs show alpha-emitting radionuclides concentrated in the areas of interstitial fibrosis.

\section{REFERENCES}

Gillett, N. A., B. L. Stegelmeier, G. Kelly, P. J. Haley, and F. F. Hahn. 1992. Expression of epidermal growth factor receptor in plutonium-239-induced lung neoplasms in dogs. Vet. Pathol 29:46-52.

Hahn, F. F., S. A. Benjamin, B. B. Boecker, T. L. Chiffelle, C. H. Hobbs, R. K. Jones, R. O. McClellan, J. A. Pickrell, and R. C. Redman. 1973. Primary pulmonary neoplasms in beagle dogs exposed to aerosols to ${ }^{144} \mathrm{Ce}$ in fused-clay particles. J. Natl. Cancer Inst. 50:675-698.

Hahn, F. F., B. A. Muggenburg, and W. C. Griffith. 1993. Primary Lung Cancer in the Longevity Study/Control Population of the ITRI Beagle Dog Colony, pp. 133-136. In: Inhalation Toxicology Research Institute Annual Report 1991-1992, G. L. Finch, K. J. Nikula, and P. L. Bradley, Eds. LMF-138, NTIS, Springfield, VA.

Harkema, J. R, S. E. Jones, D. K. Naydan, and D. W. Wilson. 1992. An atypical neuroendocrine tumor in the lung of a beagle dog. Vet. Pathol. 29:175-179.

McClellan, R. O., B. B. Boecker, F. F. Hahn, and B. A. Muggenburg. 1986. Lovelace ITRI Studies on the Toxicity of Inhaled Radionuclides in Beagle Dogs, pp. 74-96. In: Life-Span Radiation Effects Studies in Animals: What Can They Tell Us?, R. C. Thompson and J. A. Mahaffey, Eds. CONF-830951, NTIS, Springfield, VA.

Moulton, J. E. 1978. Tumors of the Respiratory System, pp. 205-239. In: Tumors in Domestic Animals, J. E. Moulton, Ed. University of California Press, Berkeley, CA.

Moulton, J. E., C. von Tschamer, and R. Schneider. 1981. Classification of lung carcinomas in the dog and cat. Vet. Pathol. 18:513-528.

Park, J. F., G. E. Dagle, H. A. Ragan, R. E. Weller, and D. L. Stevens. 1986. Current Status of Life-Span Studies with Inhaled Plutonium in Beagles at Pacific Northwest Laboratory, pp. 455470. In: Life-Span Radiation Effects Studies in Animals: What Can They Tell Us?, R. C.

Thompson and J. A. Mahaffey, eds. CONF-830951, NTIS, Springfield, VA.

Slauson, D.O., F.F. Hahn, S.A. Benjamin, T.L. Chiffelle, and R.K. Jones. 1976. Inflammatory sequences in acute pulmonary radiation injury. Am. J. Pathol. 82:549-572.

Slauson, D.O., F.F. Hahn, and T.L. Chiffelle. 1977. The pulmonary vascular pathology of experimental radiation pneumonitis. Am. J. Pathol. 88:635-654.

World Health Organization. International Histologic Classification of Tumours in Domestic Animals, Bulletin WHO 50:1-144 (1974) and 50:145-282 (1976) WHO, Geneva, Switzerland. 


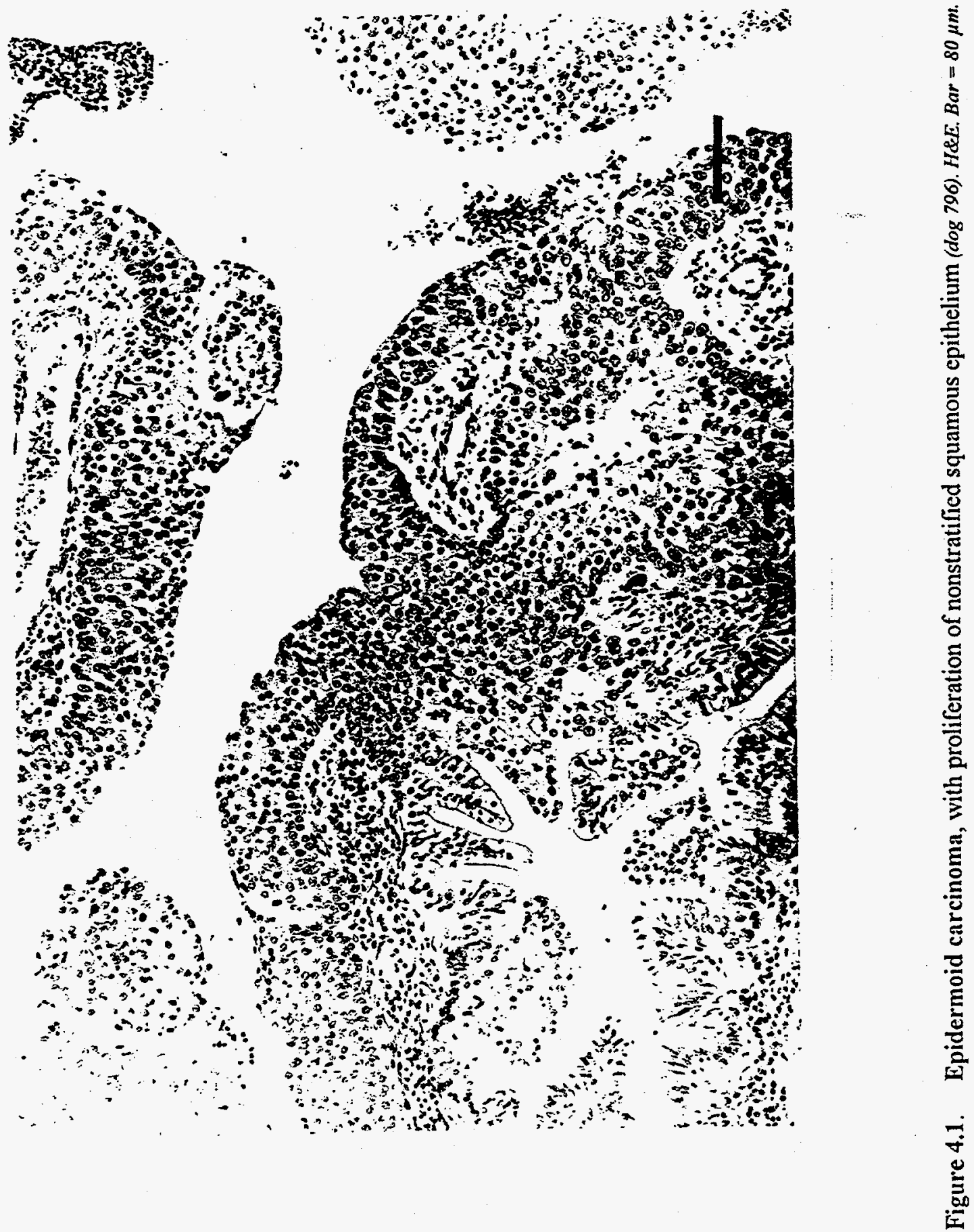




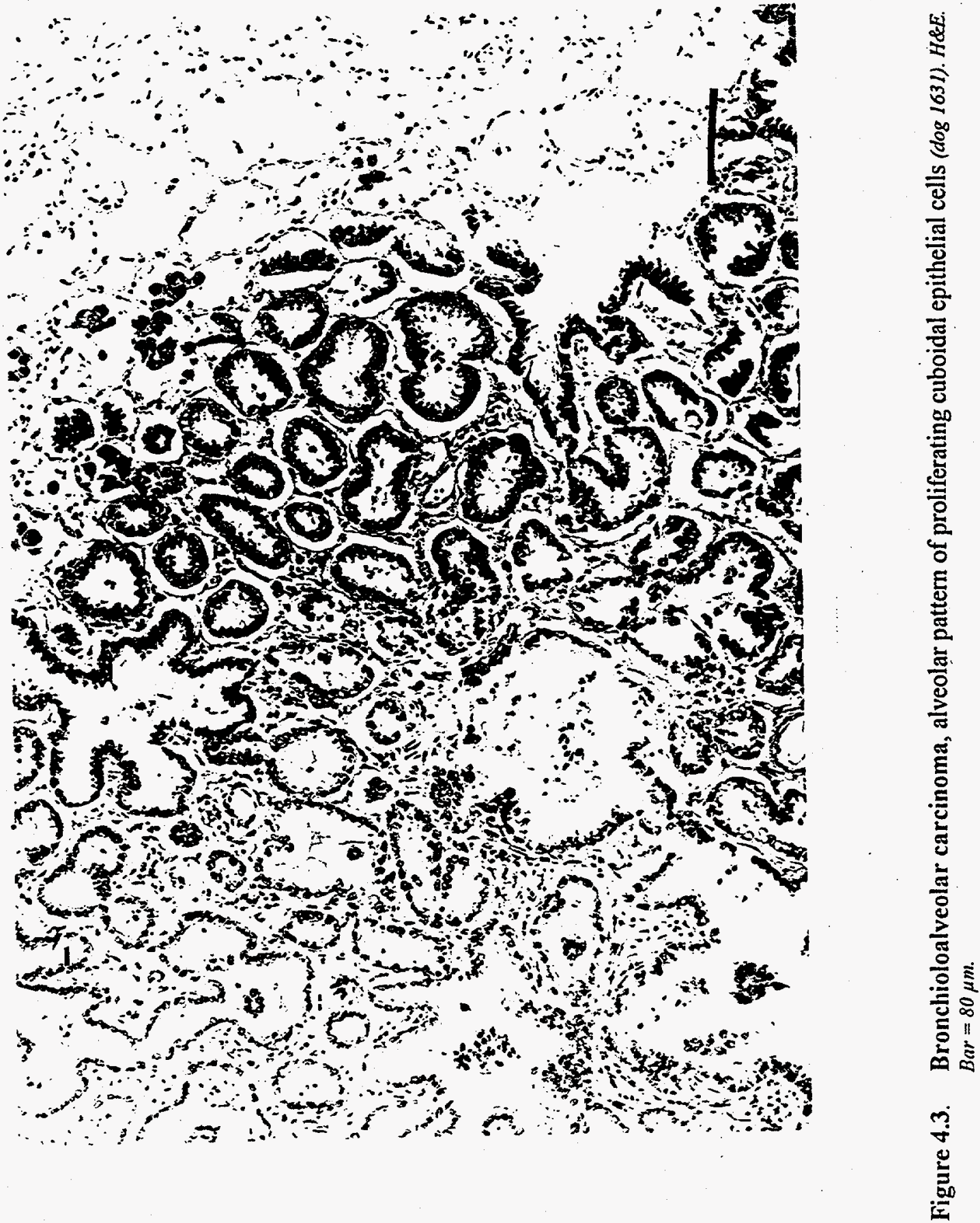


4.3

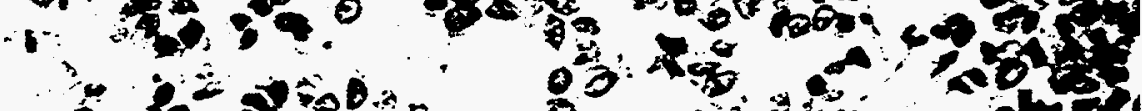

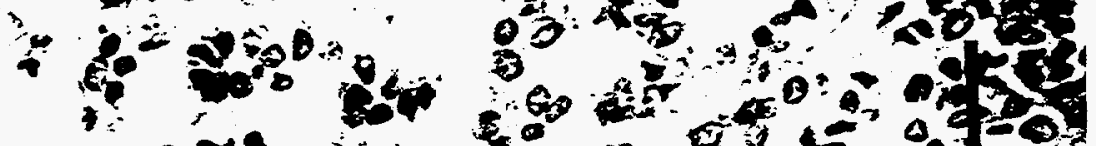

$i$ or, -0 - 15

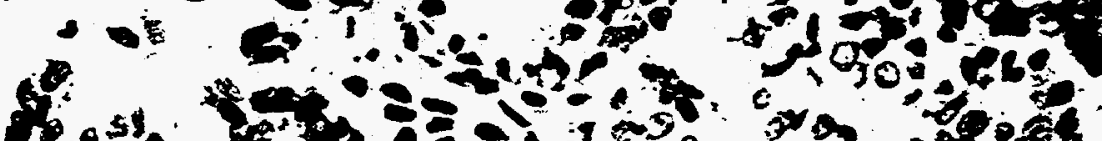

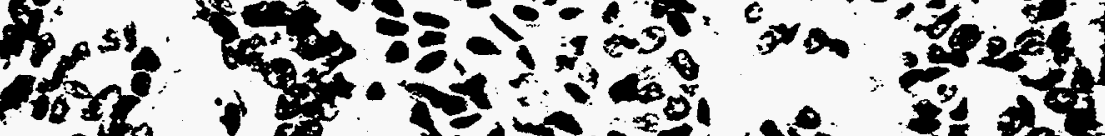

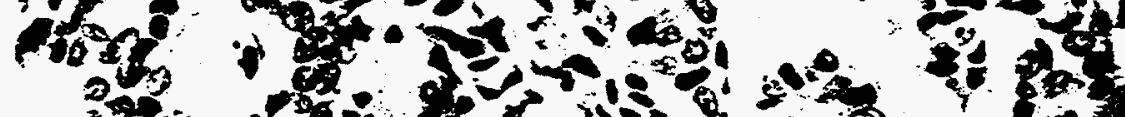

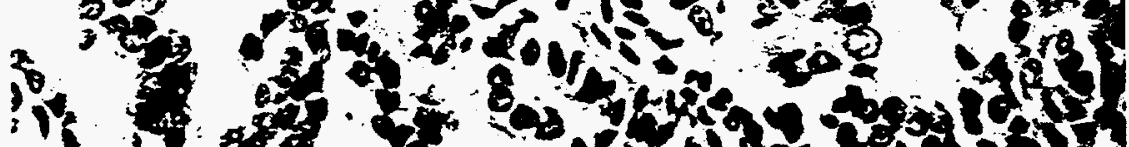

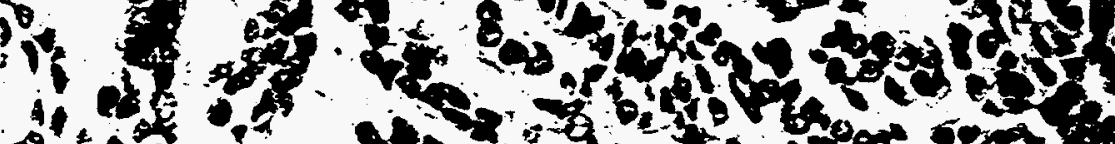

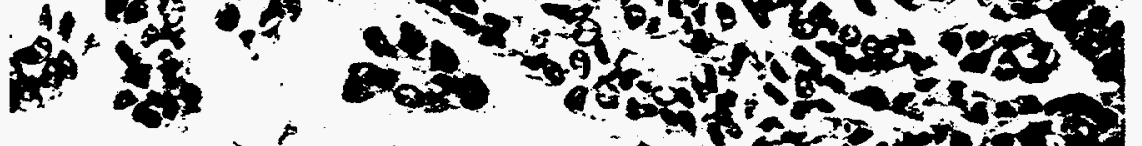

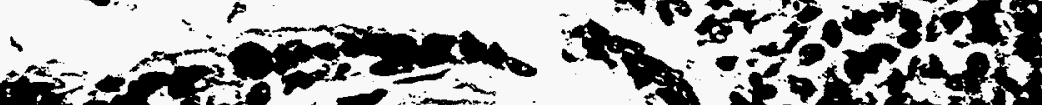

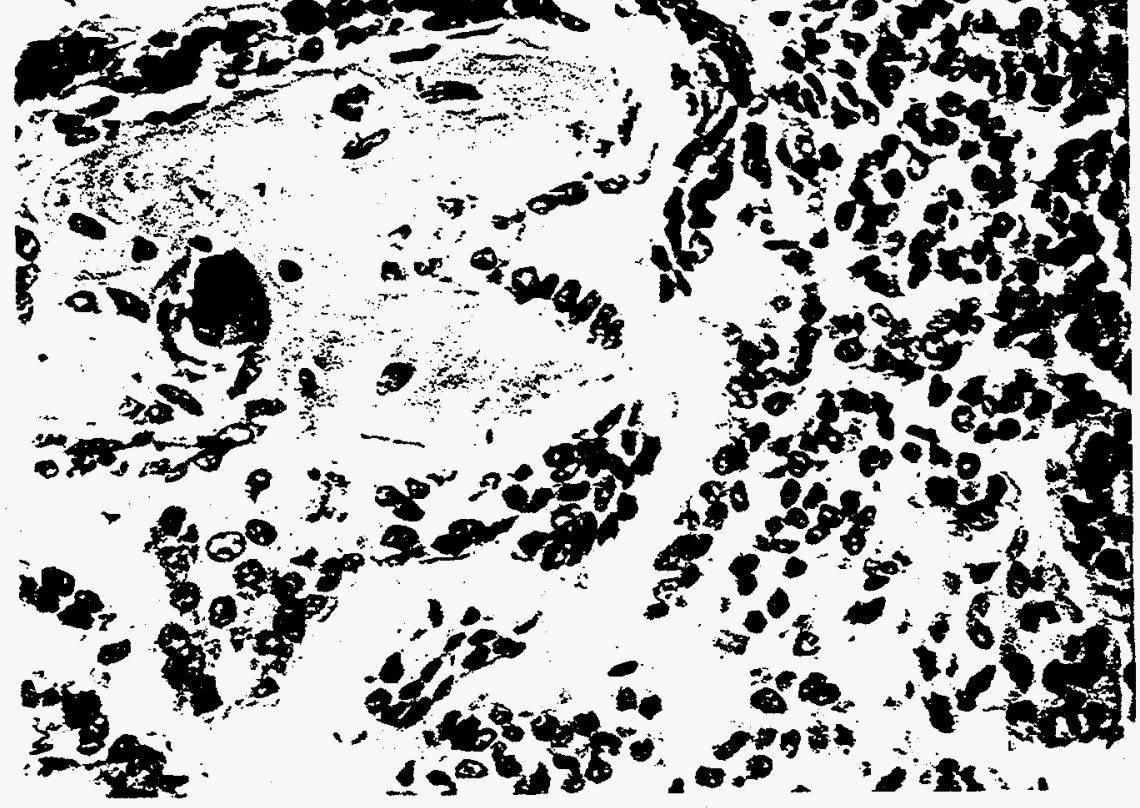




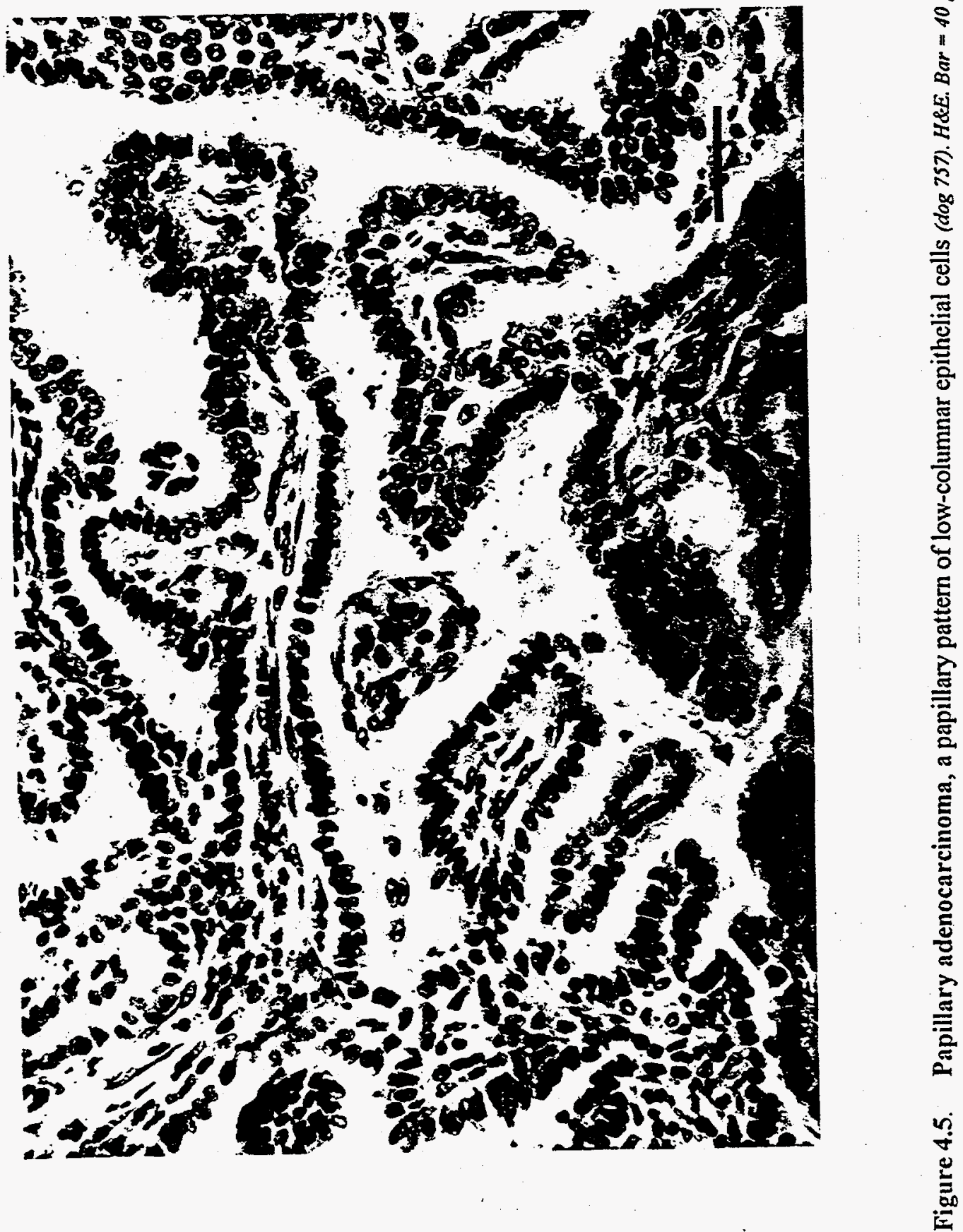




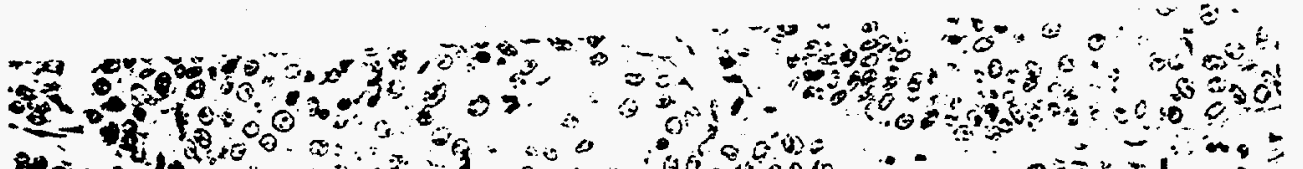

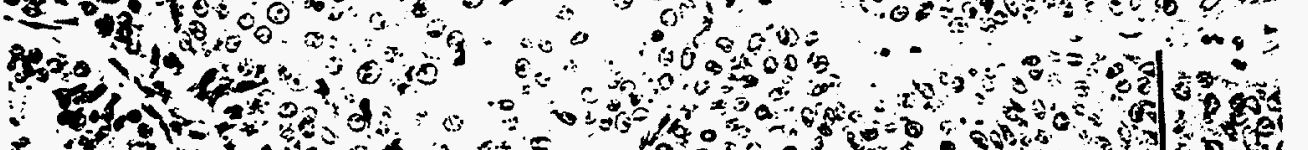
3.

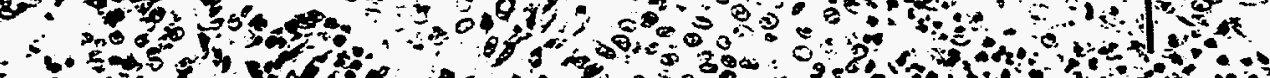

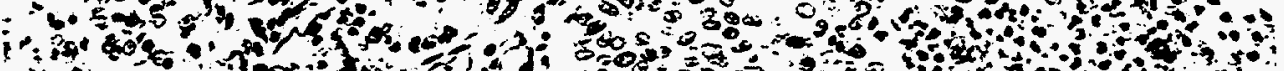

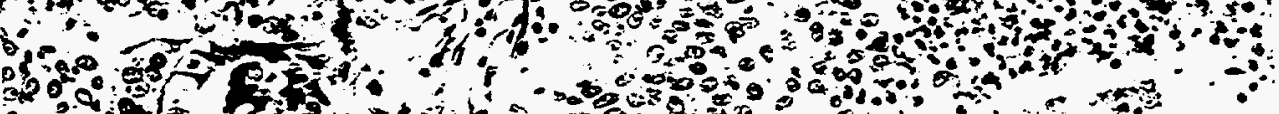
\% 5 a tol $\because$ of Ao 90 \% 6. Hog Ha

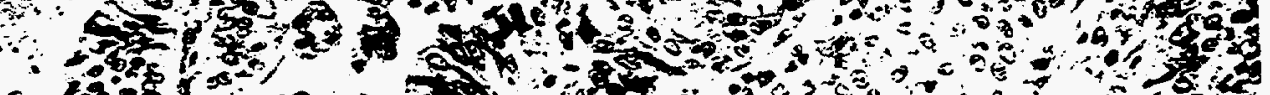

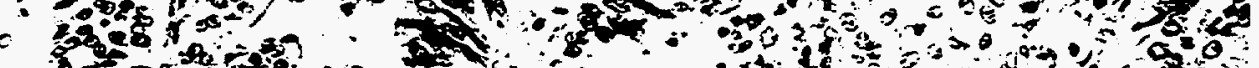
- 0 a

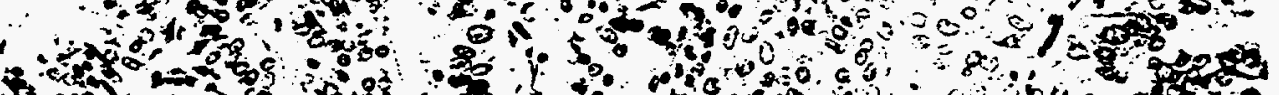
30 , $\therefore$ as and 3.

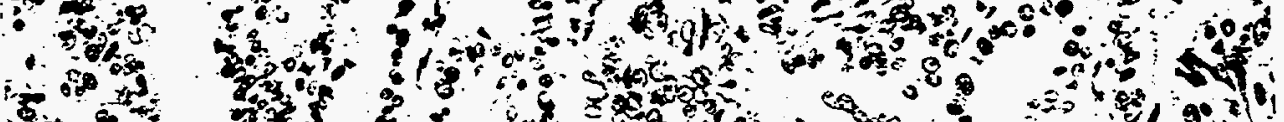

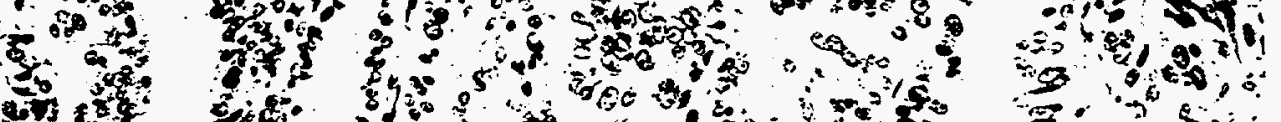
5y

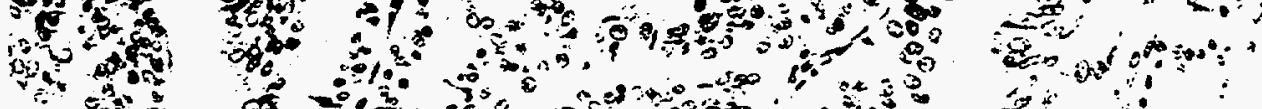

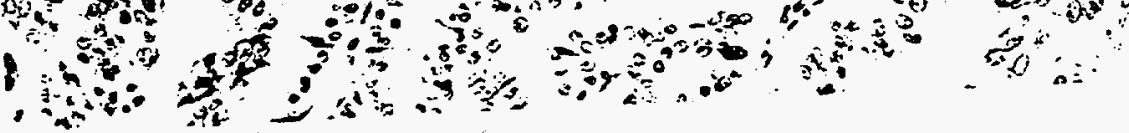




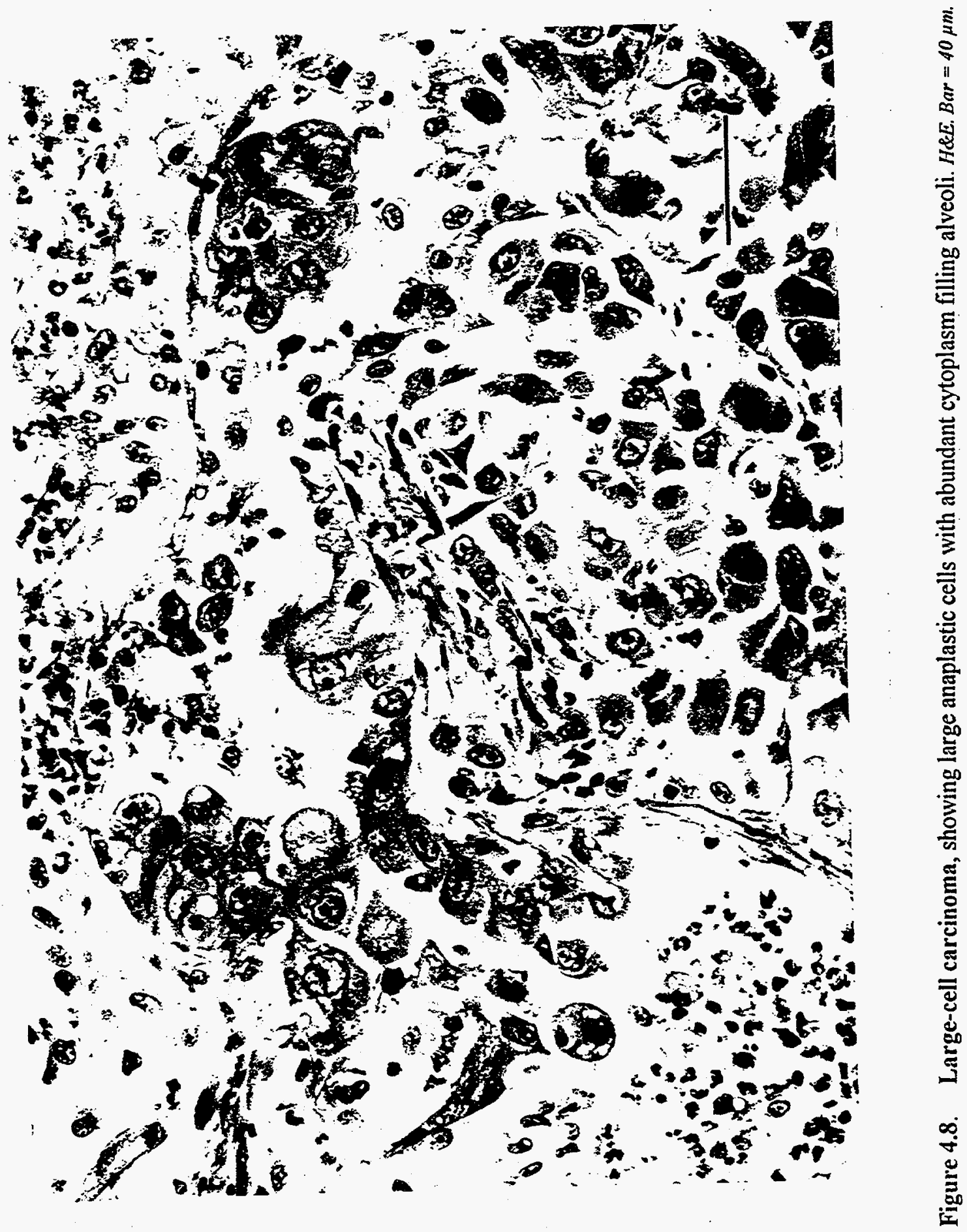




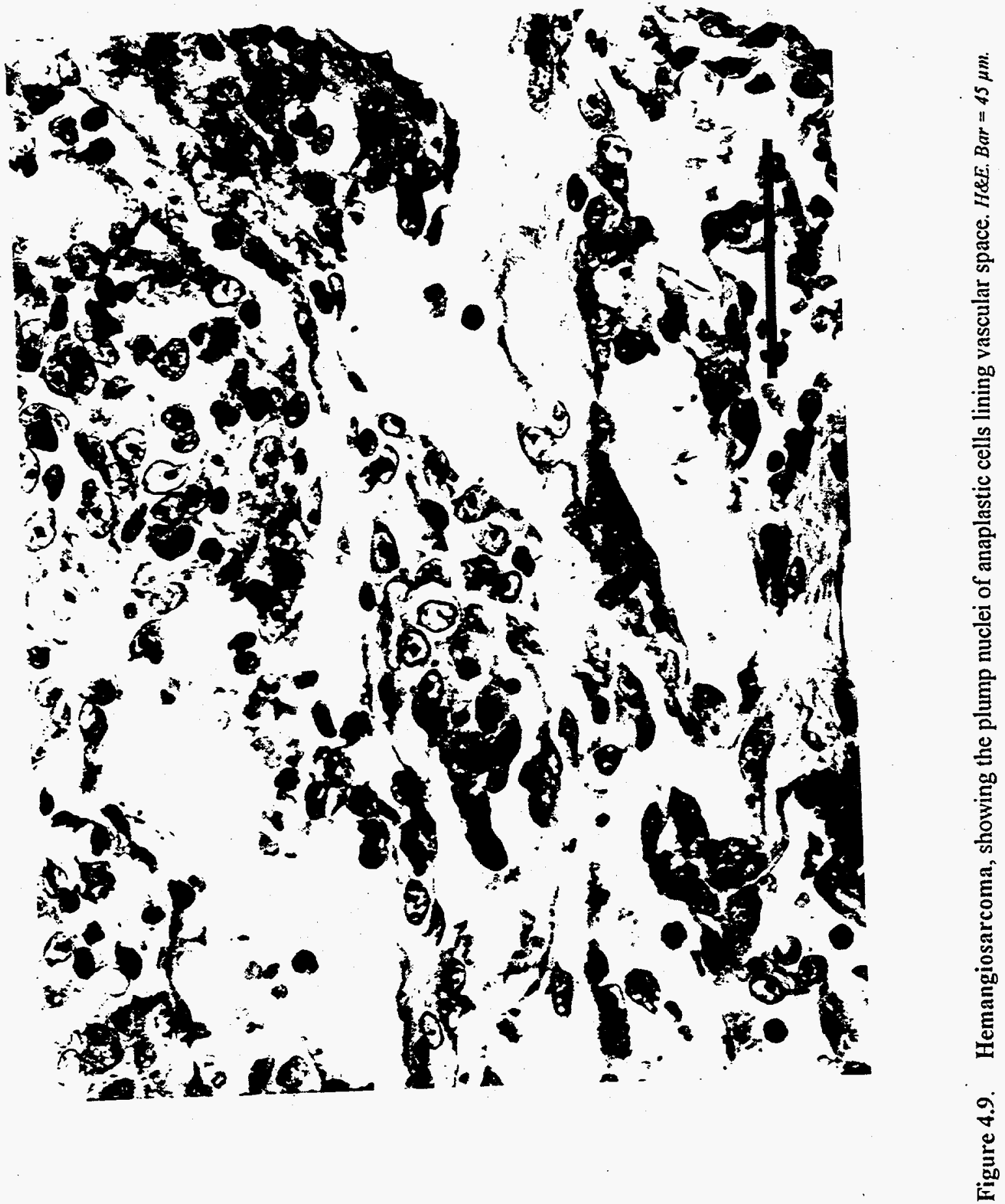




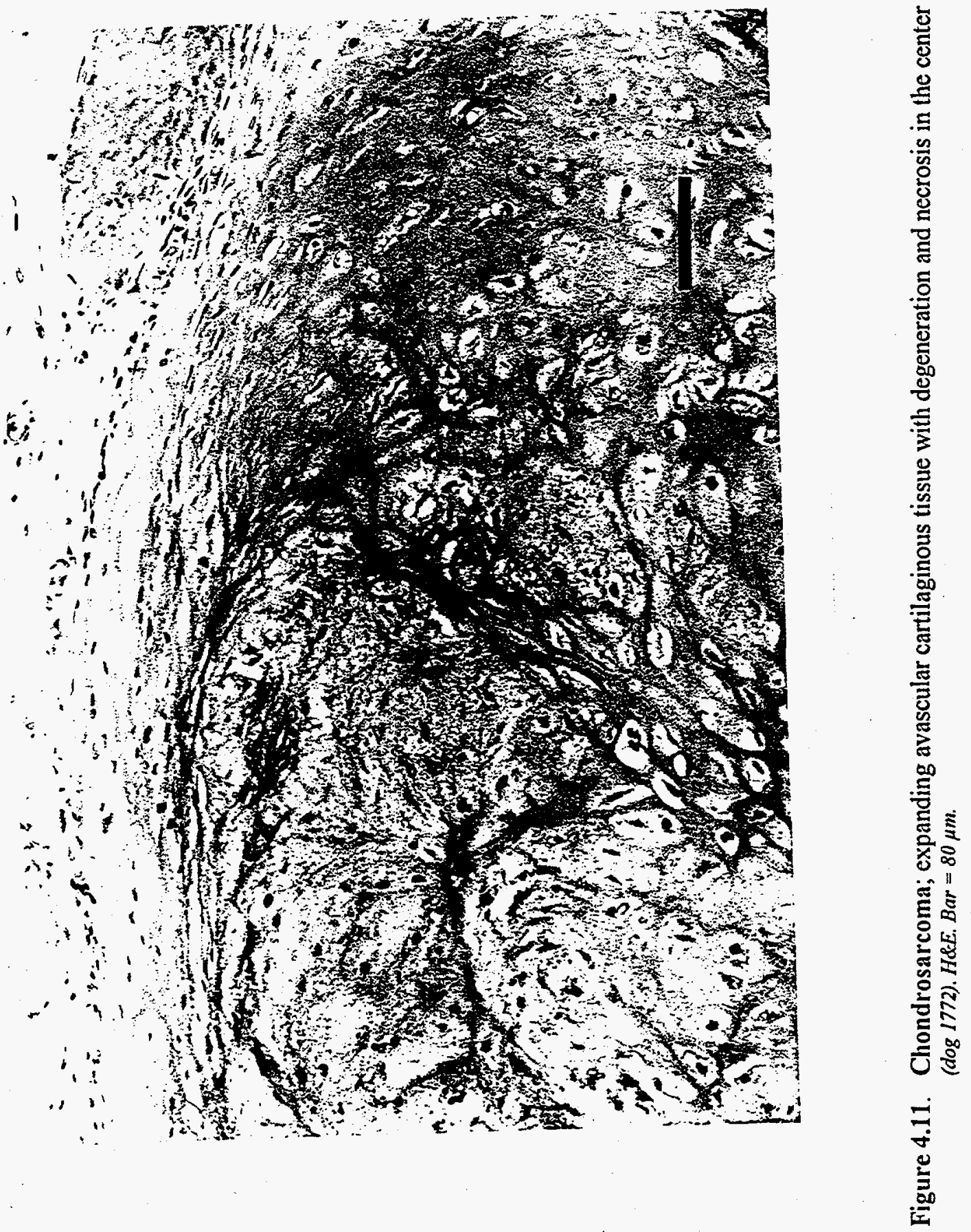




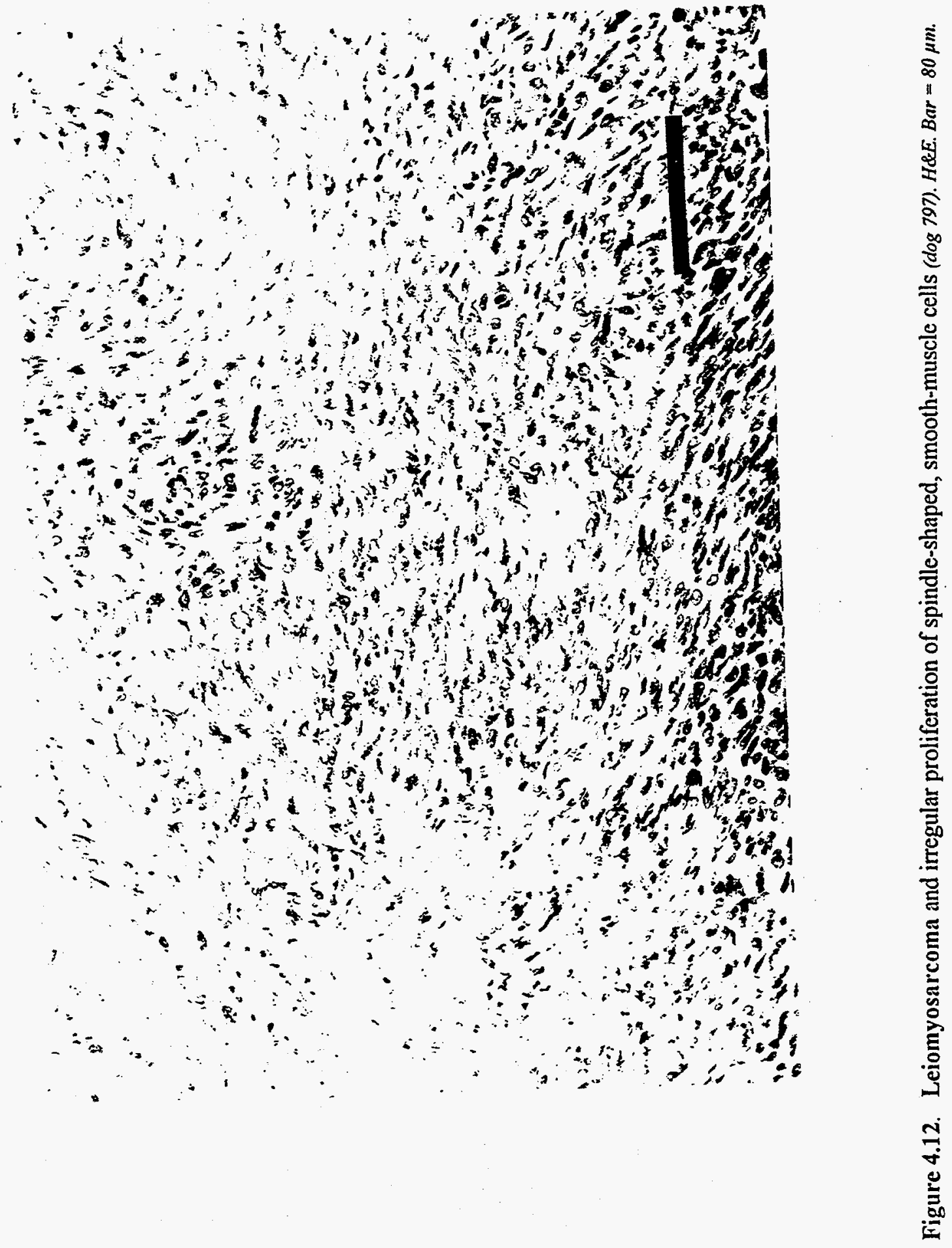




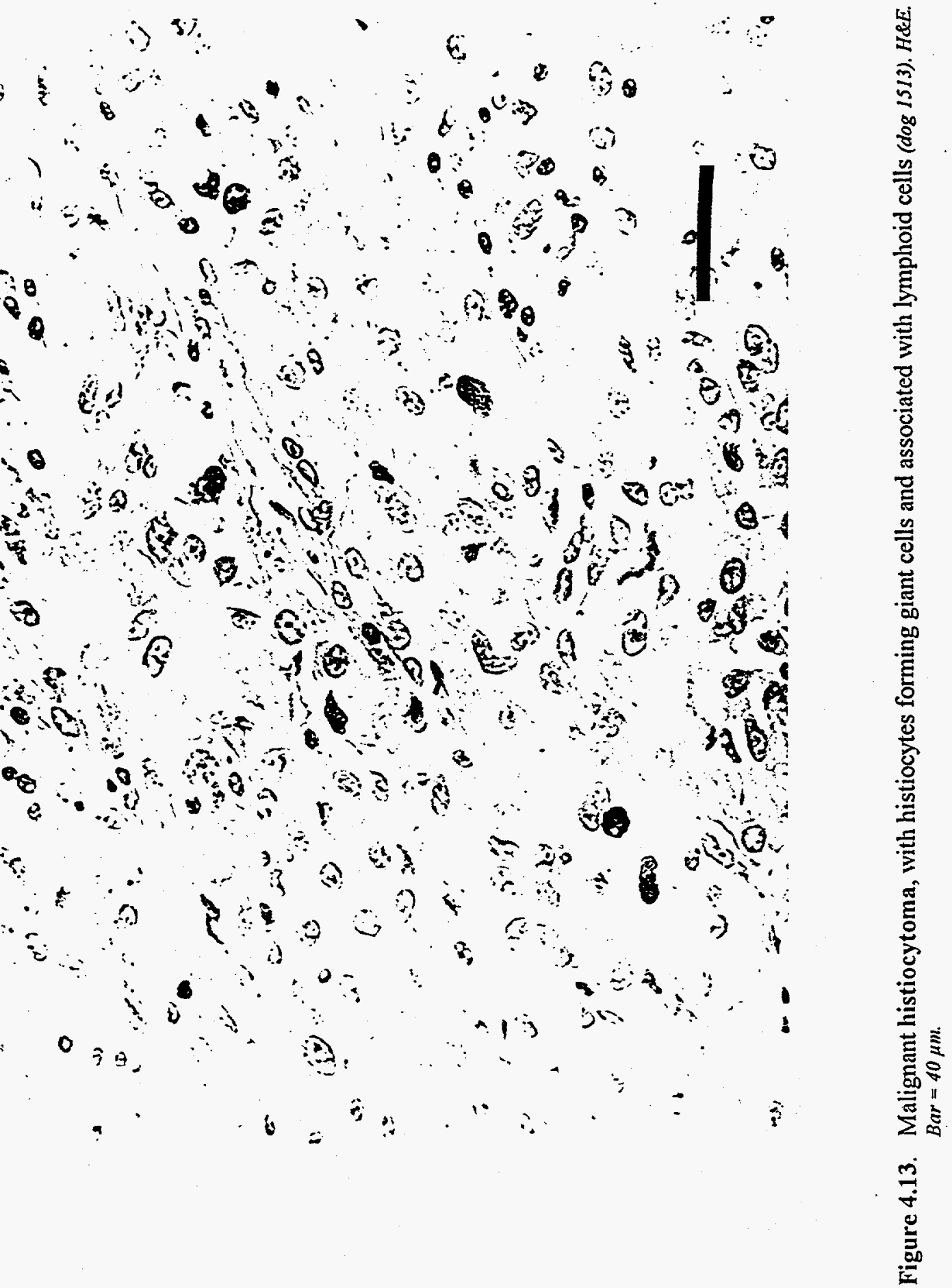




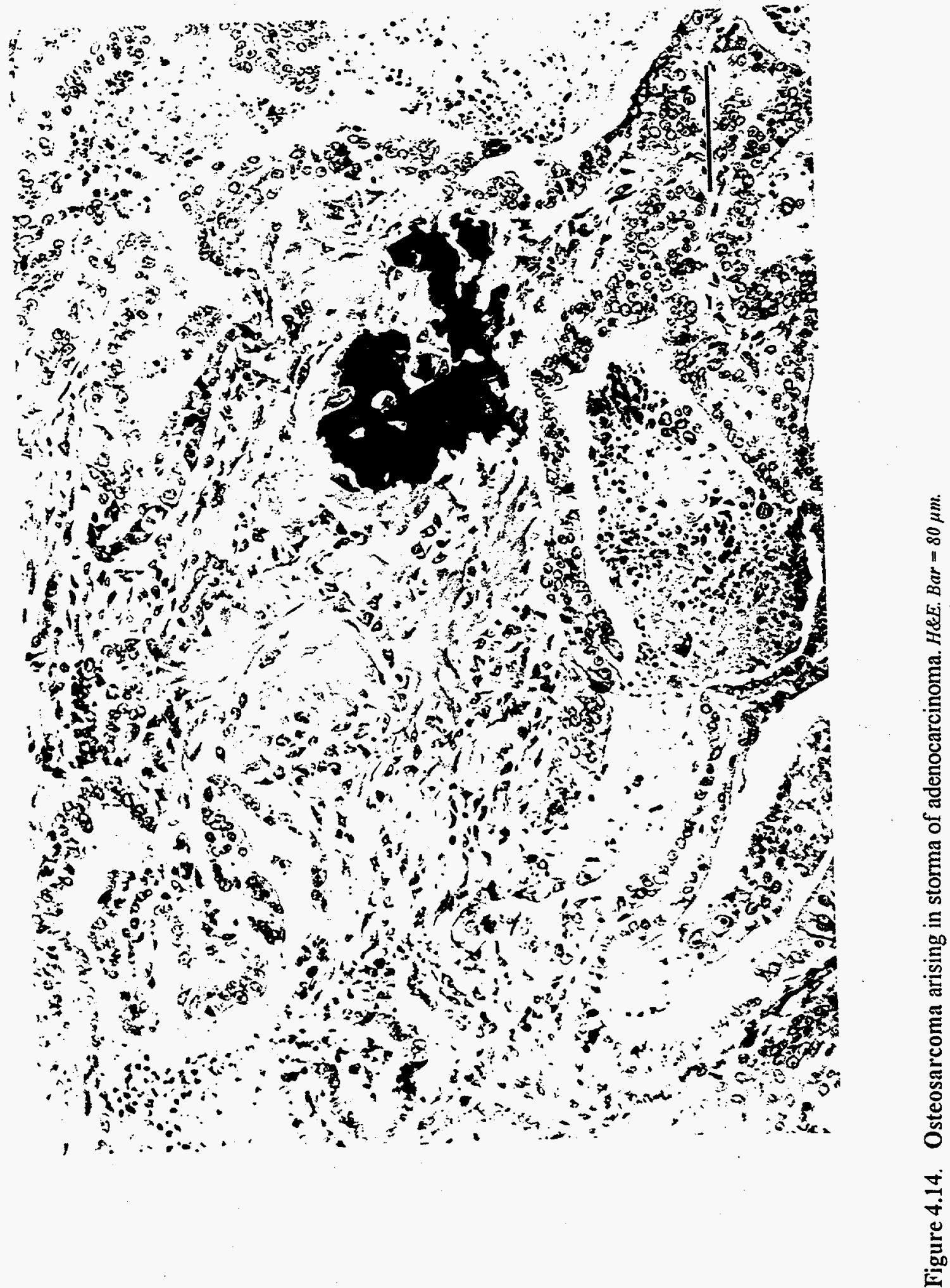




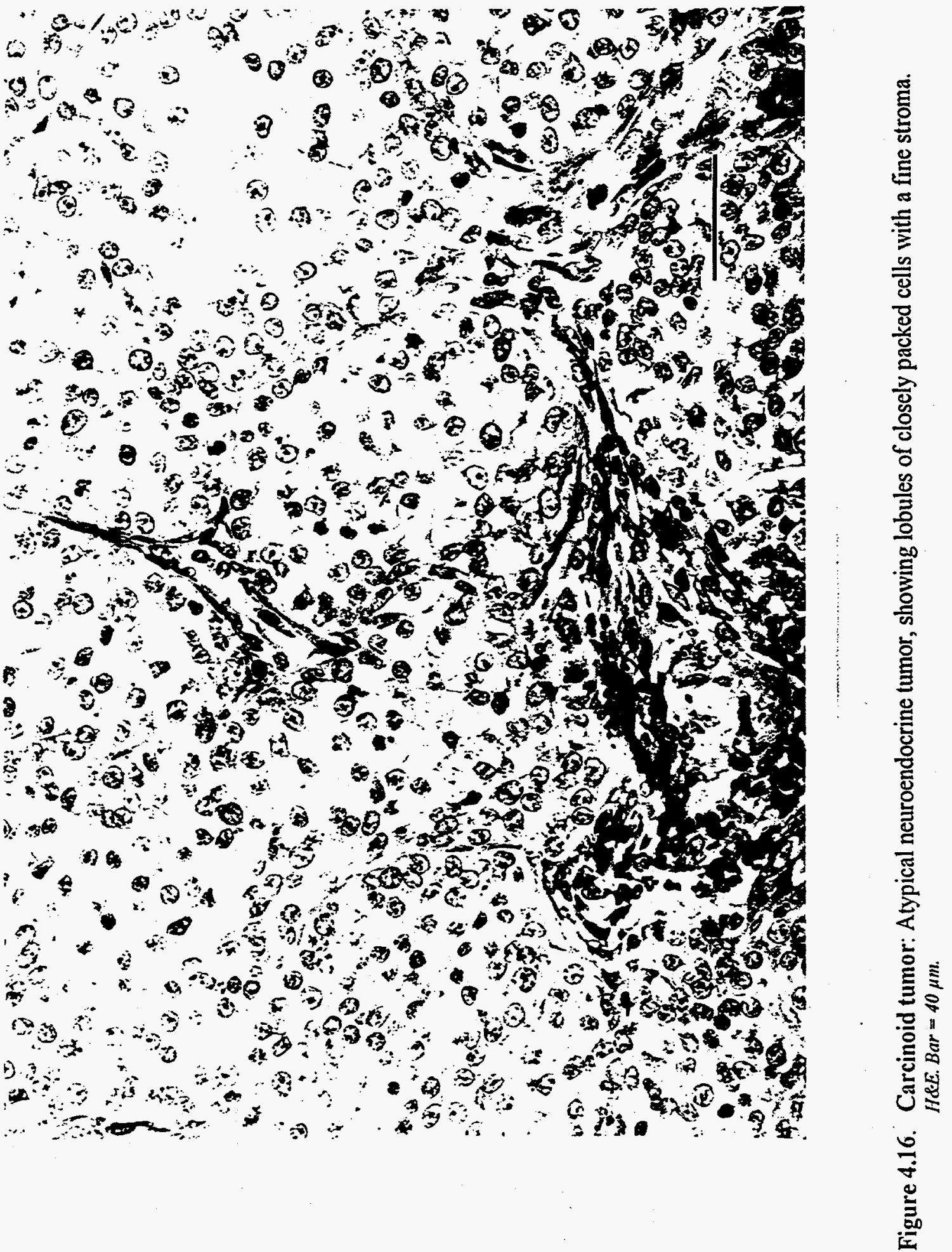




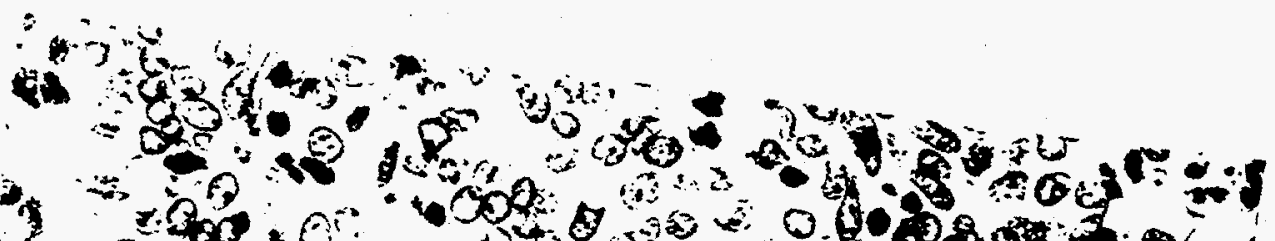

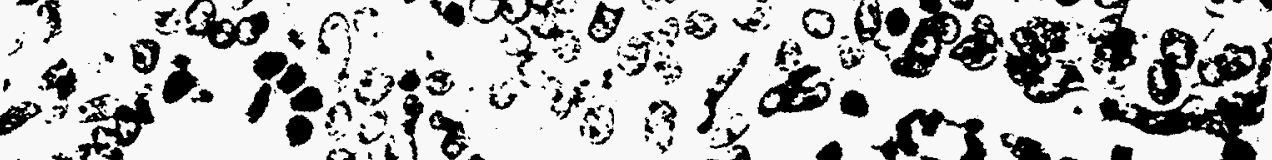

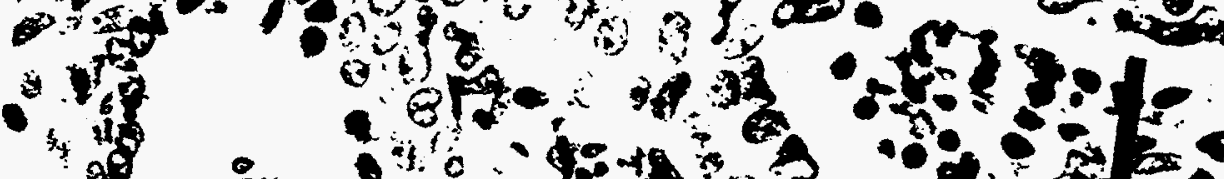

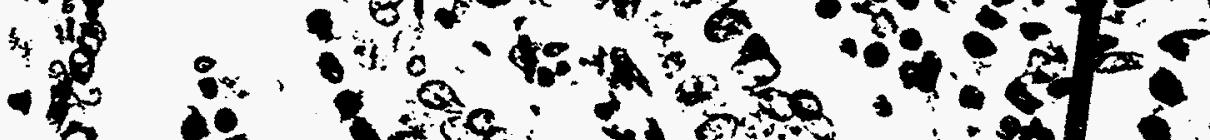

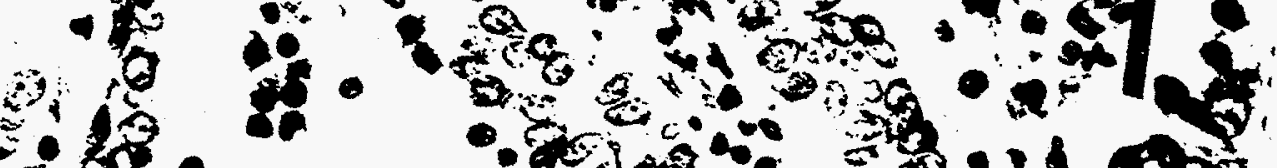

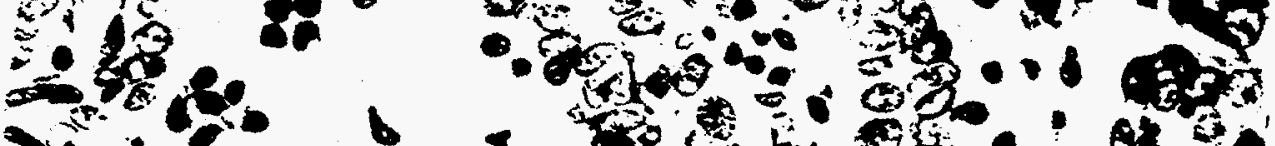

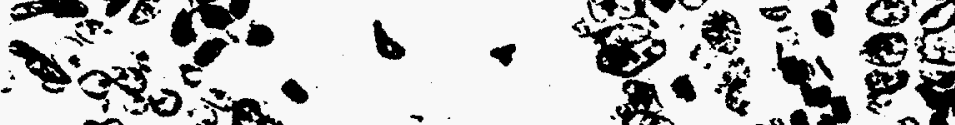

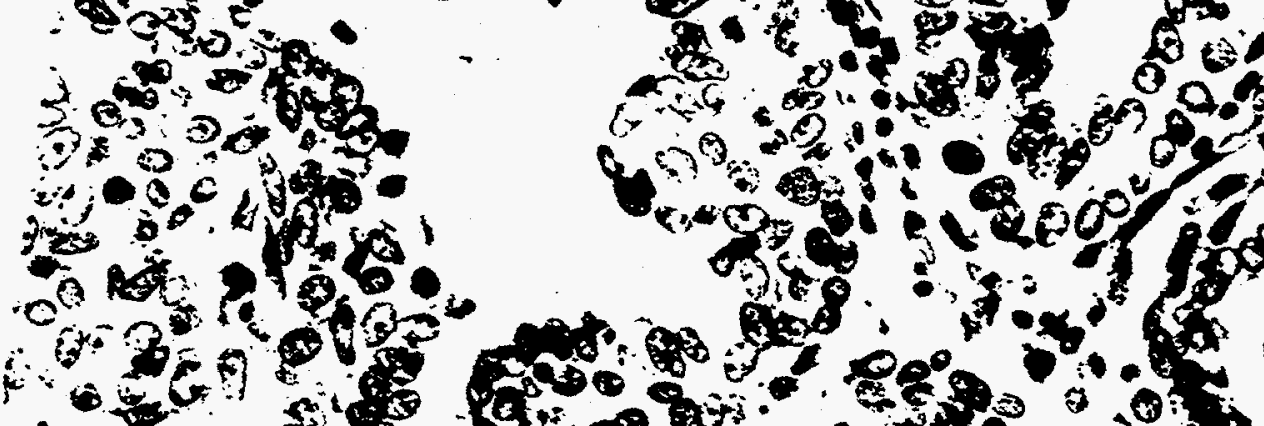

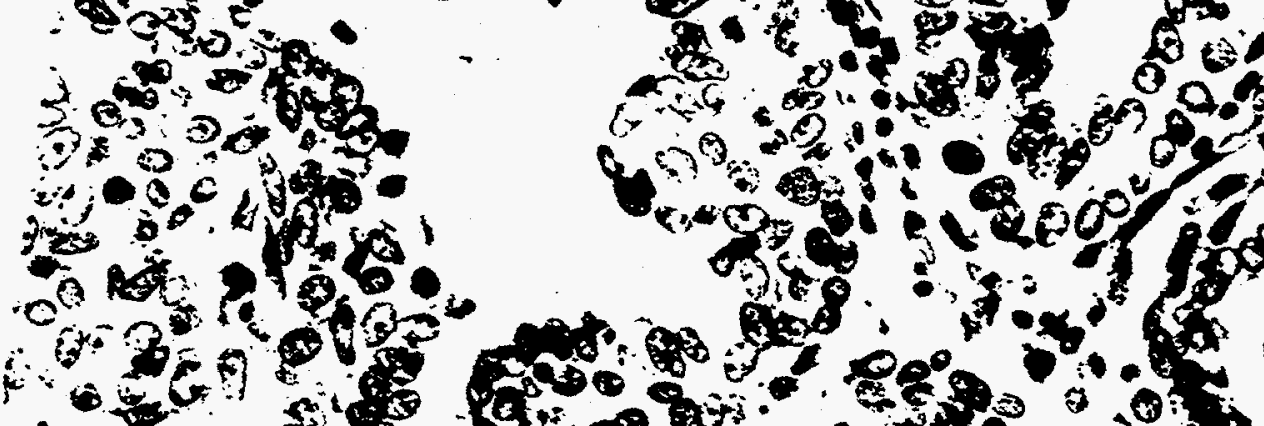

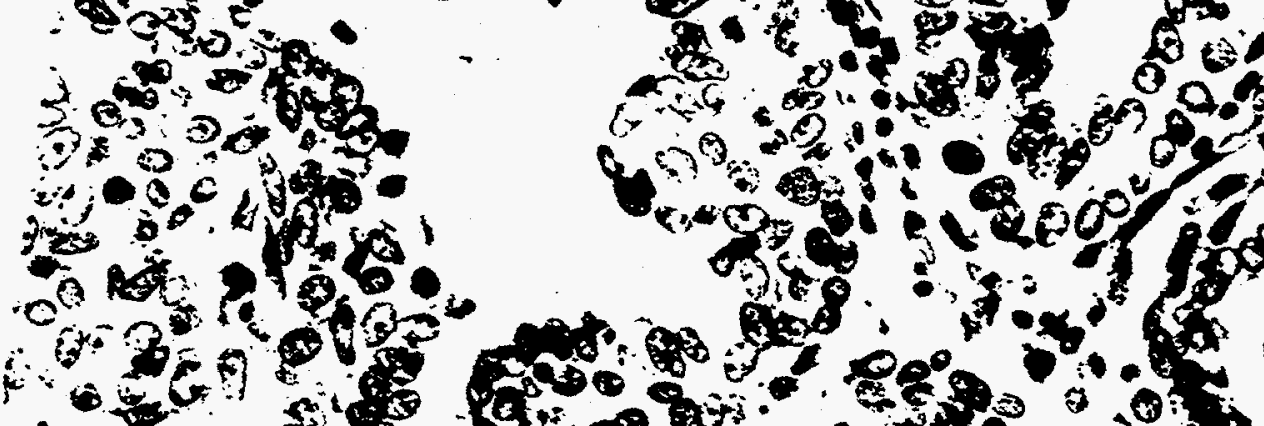

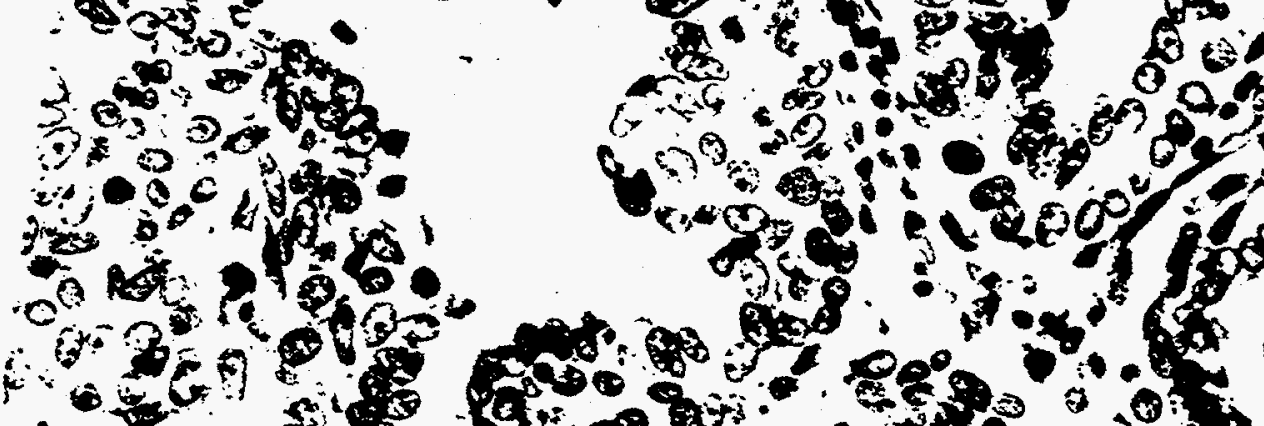

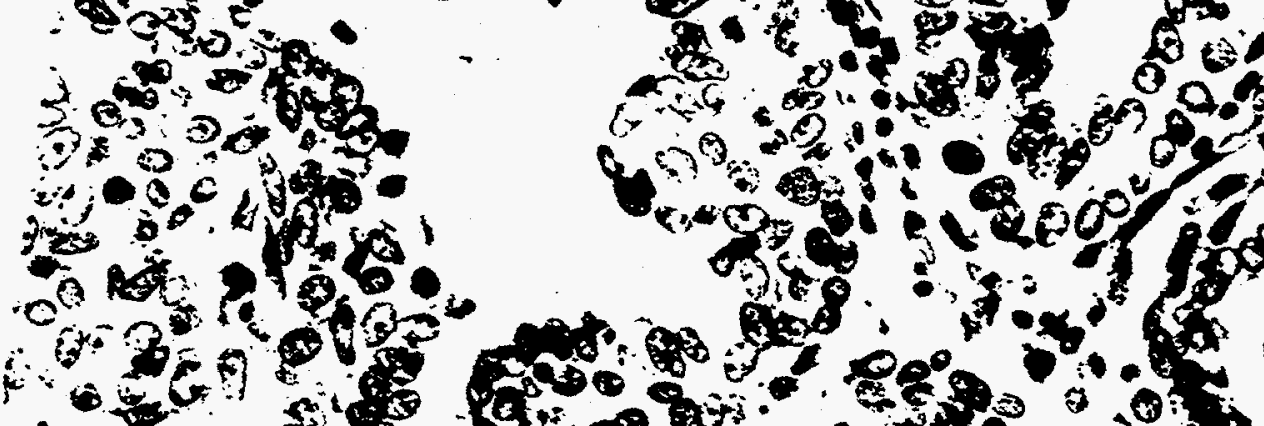
6.4.

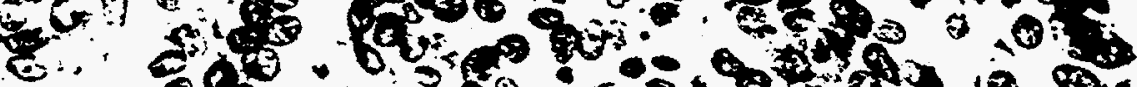

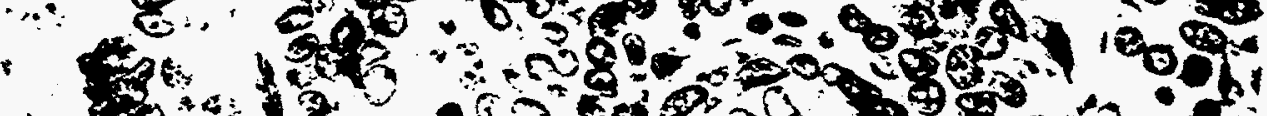

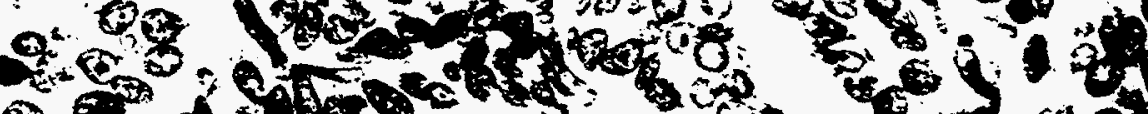

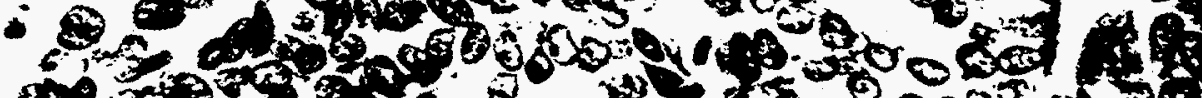

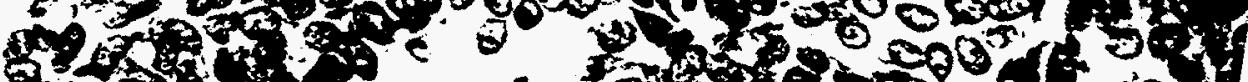

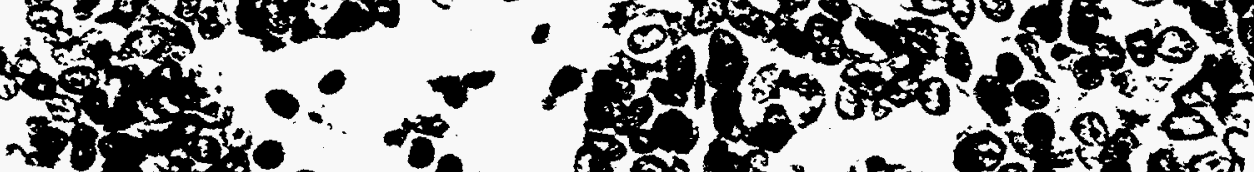

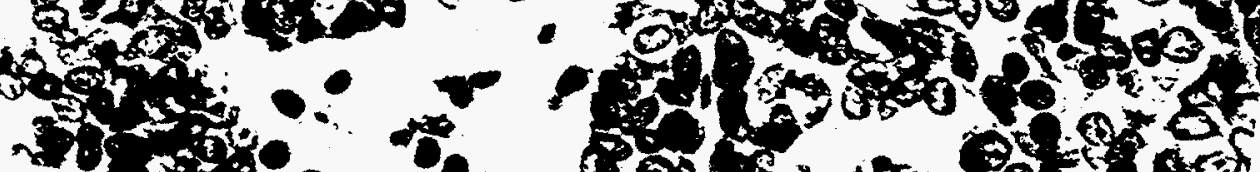

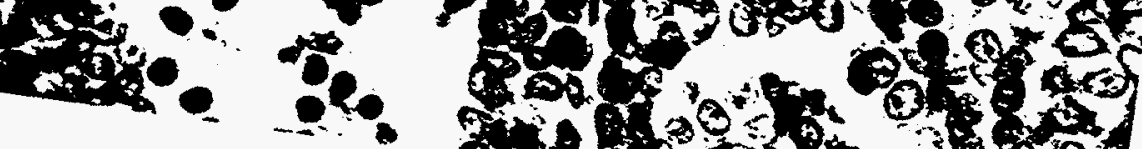

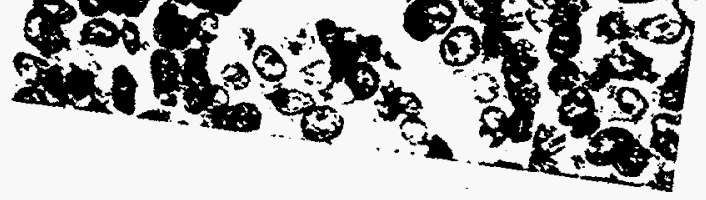

i 


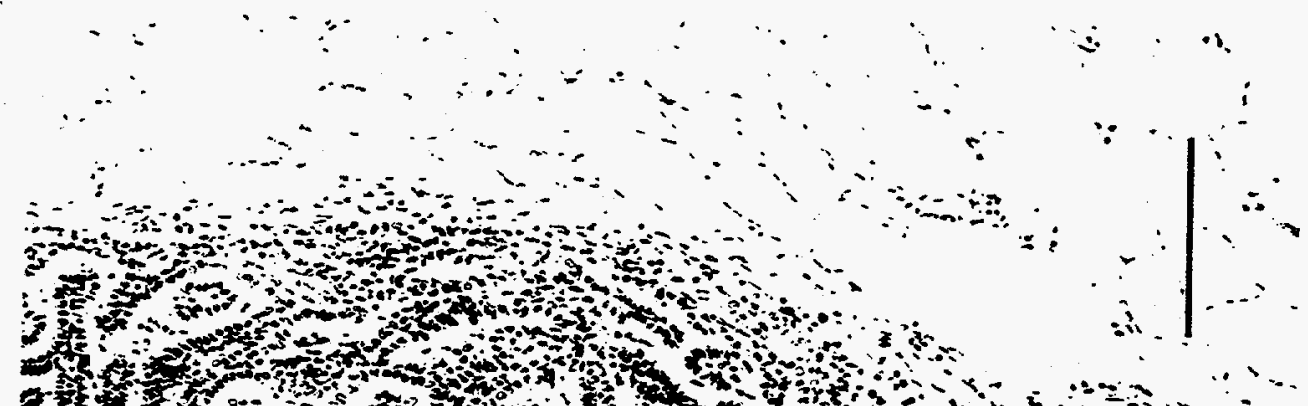

arta

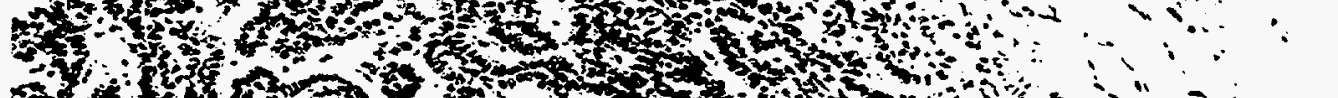

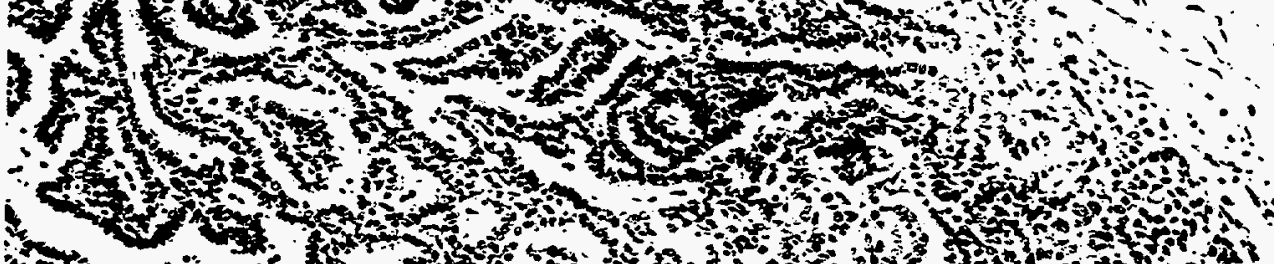

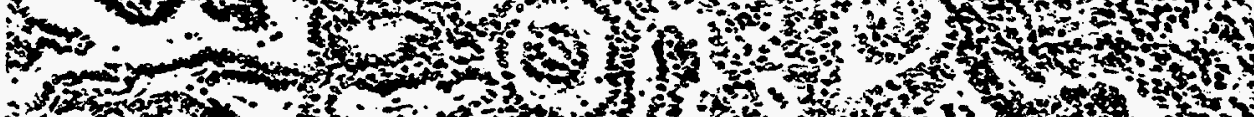
30 .

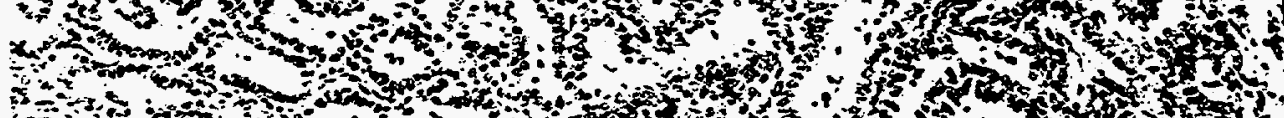
-.

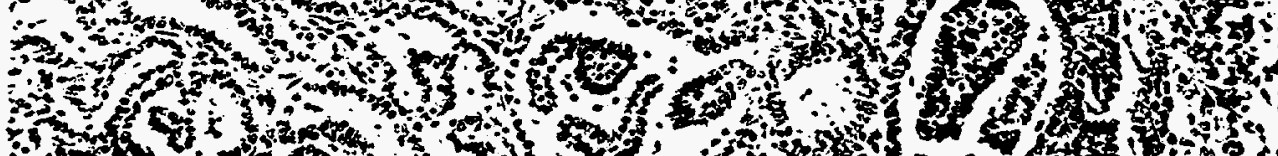

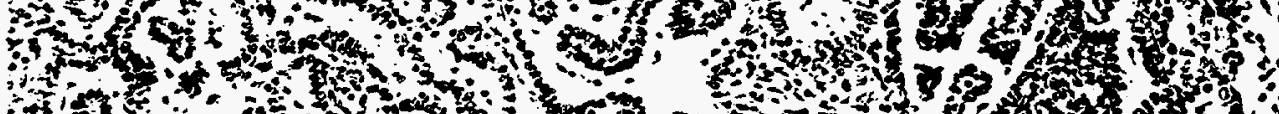
- 1 -

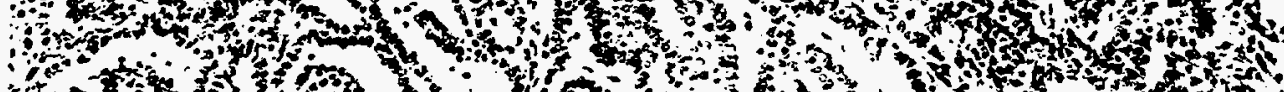
to Fof

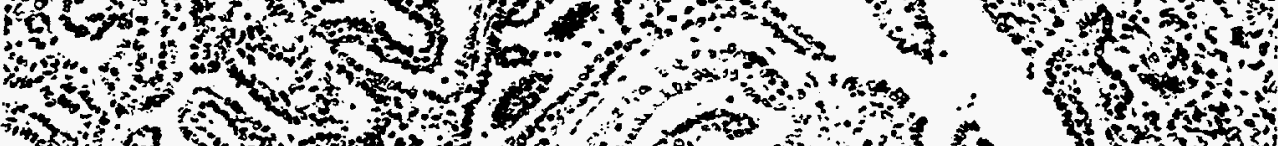

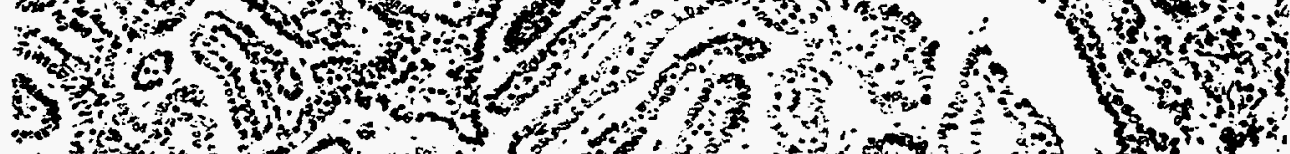
W.

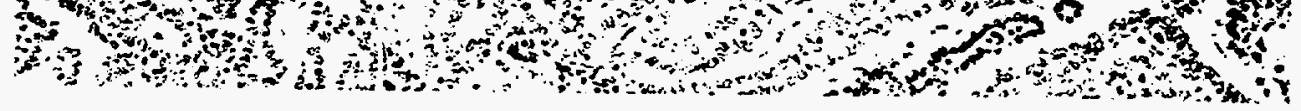

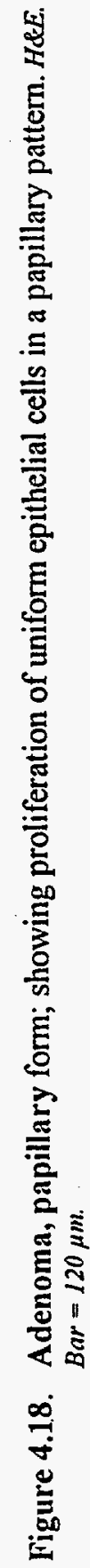




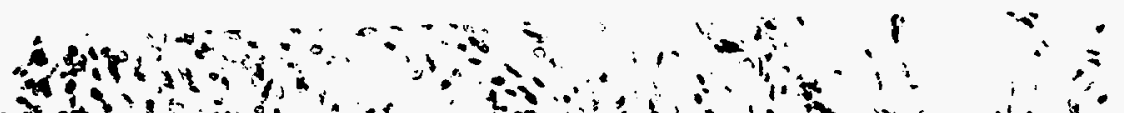
(18)

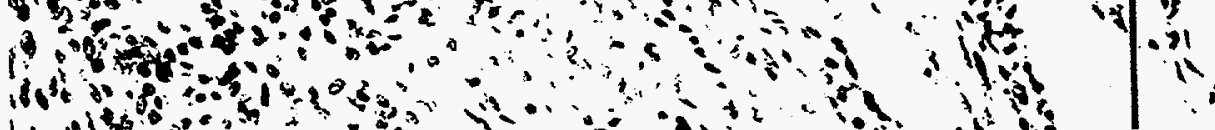

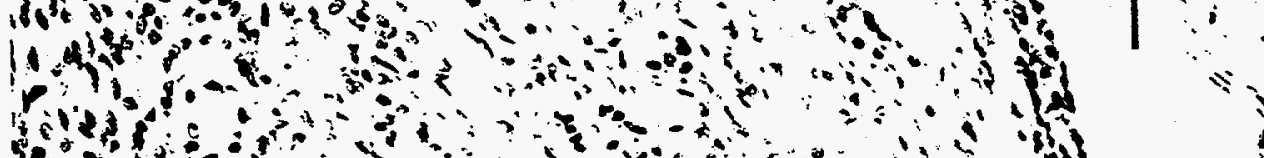
tol

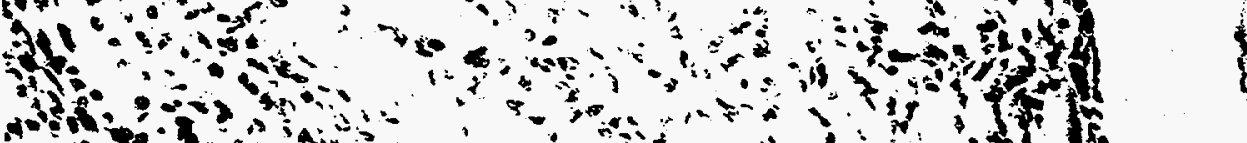
Iflos

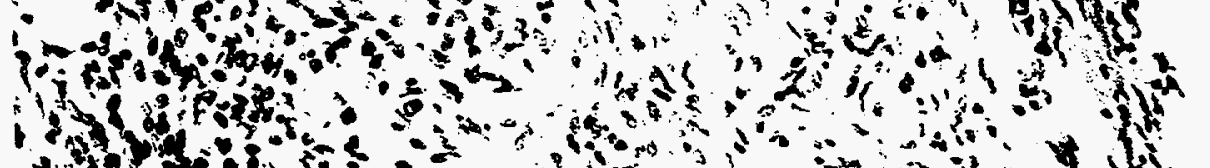
(3)

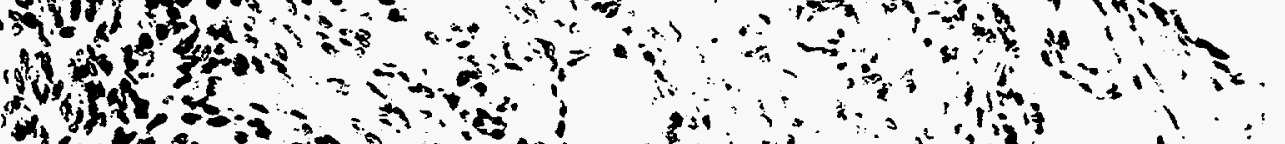
in

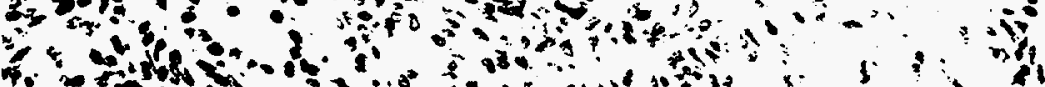
of

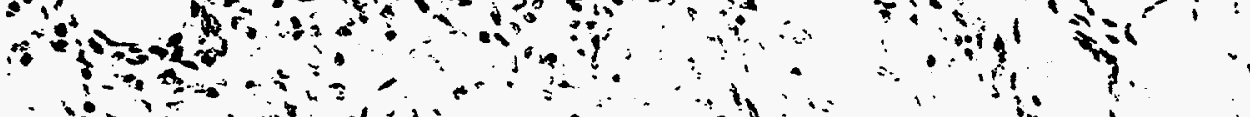
$\therefore$ a

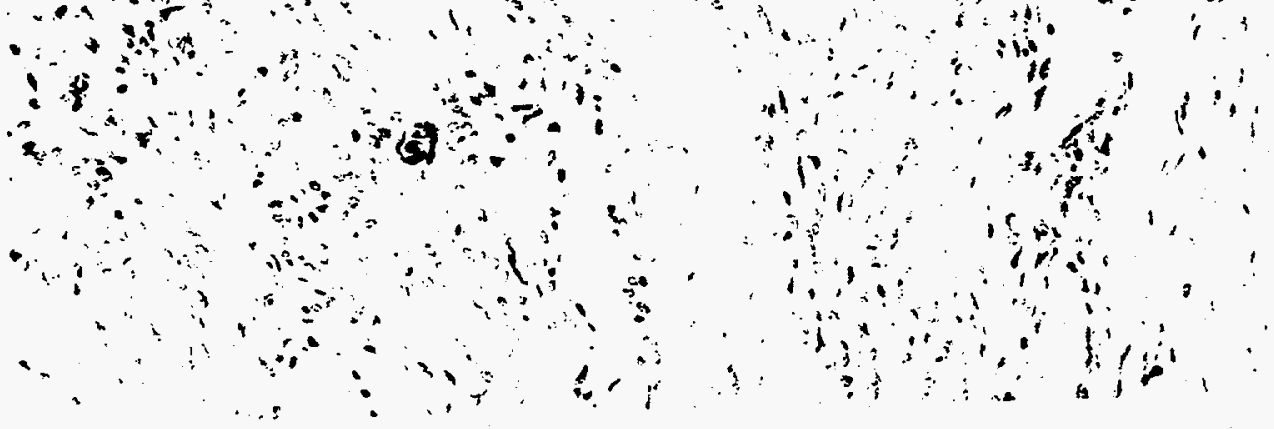




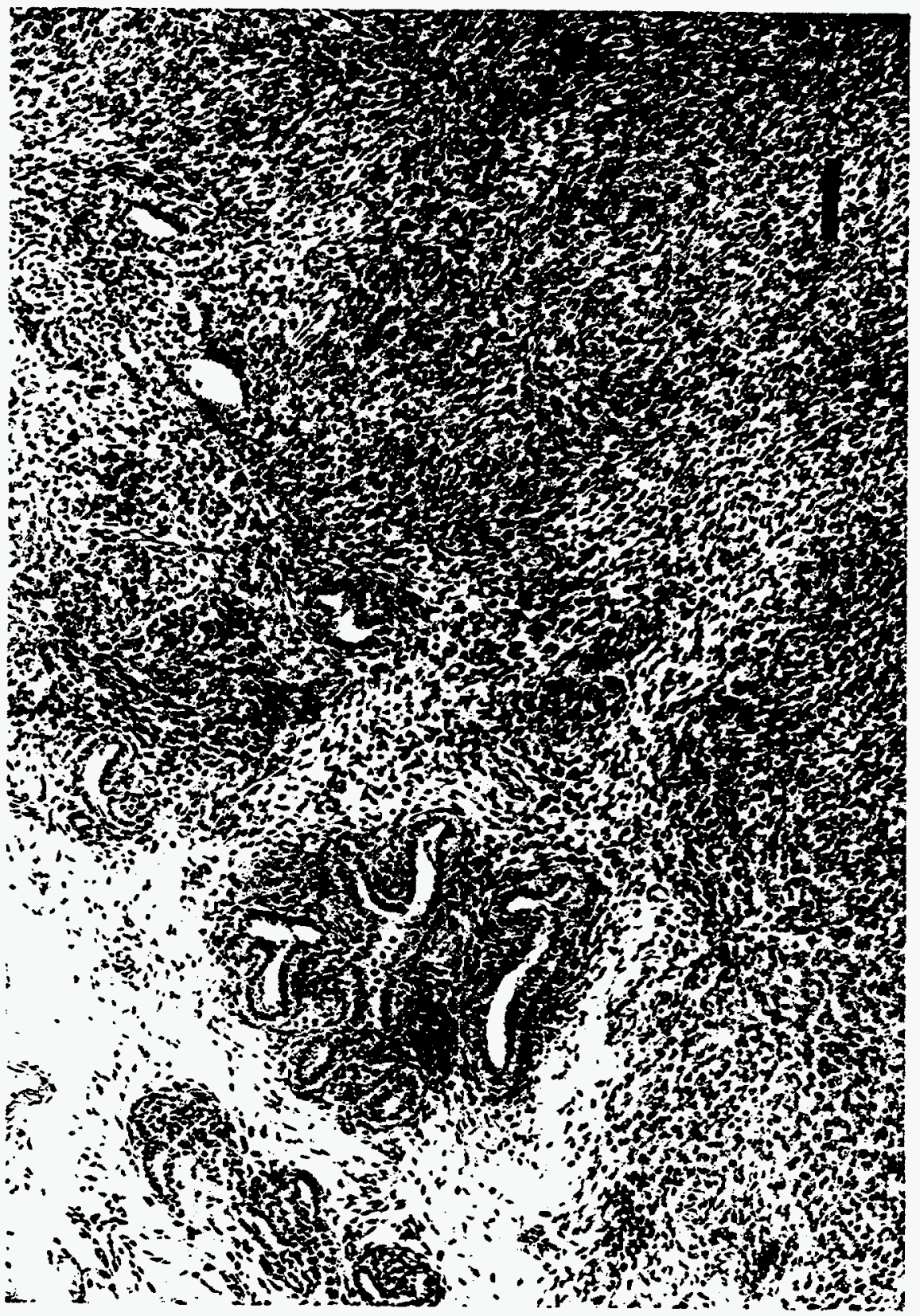




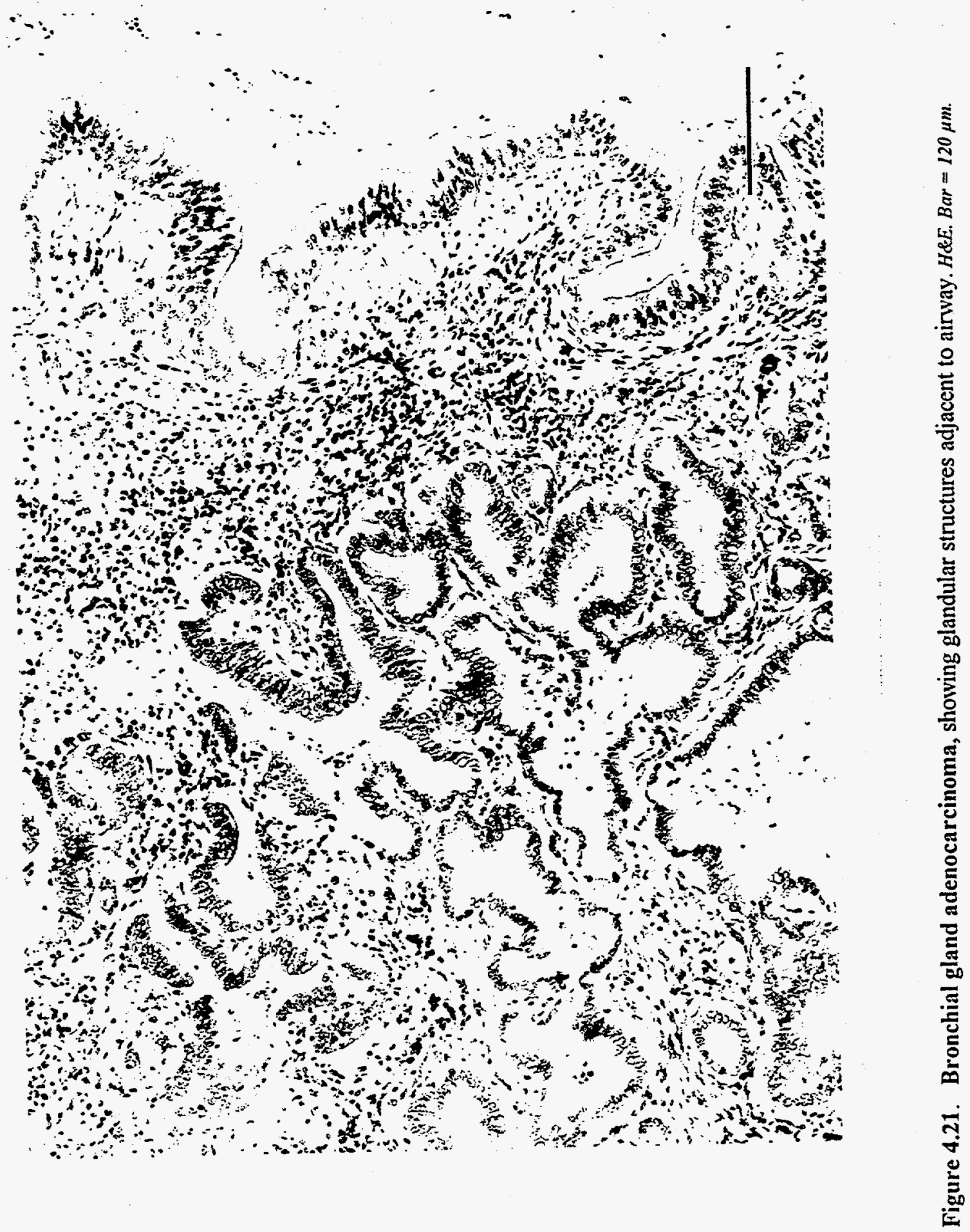




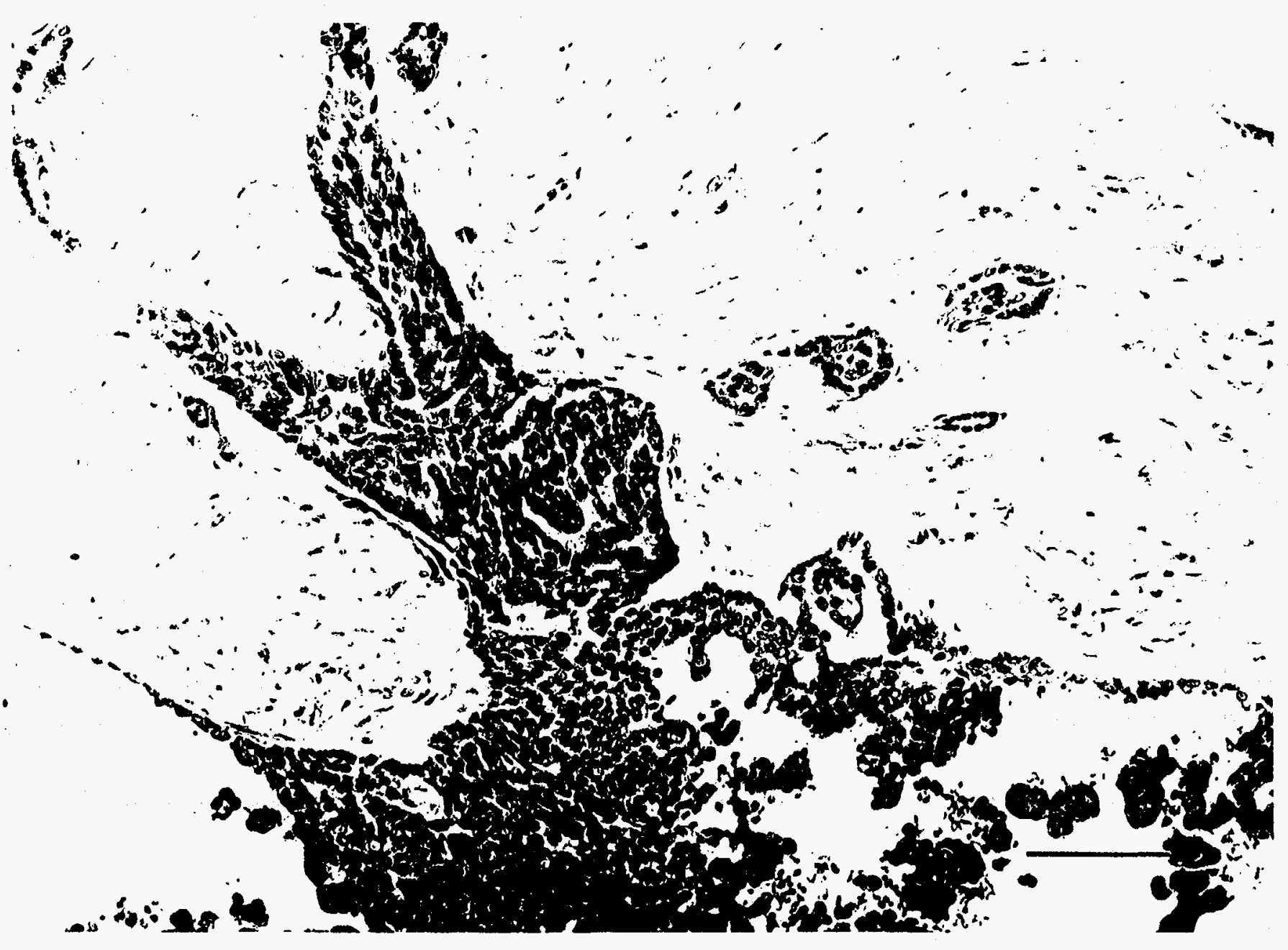

Figure 4.22. Mesothelioma, malignant, showing papillary proliferation of anaplastic mesothelial cells and induction of fibrosis (dog 1222), $H \& E$. Bar $=80 \mu \mathrm{m}$. 


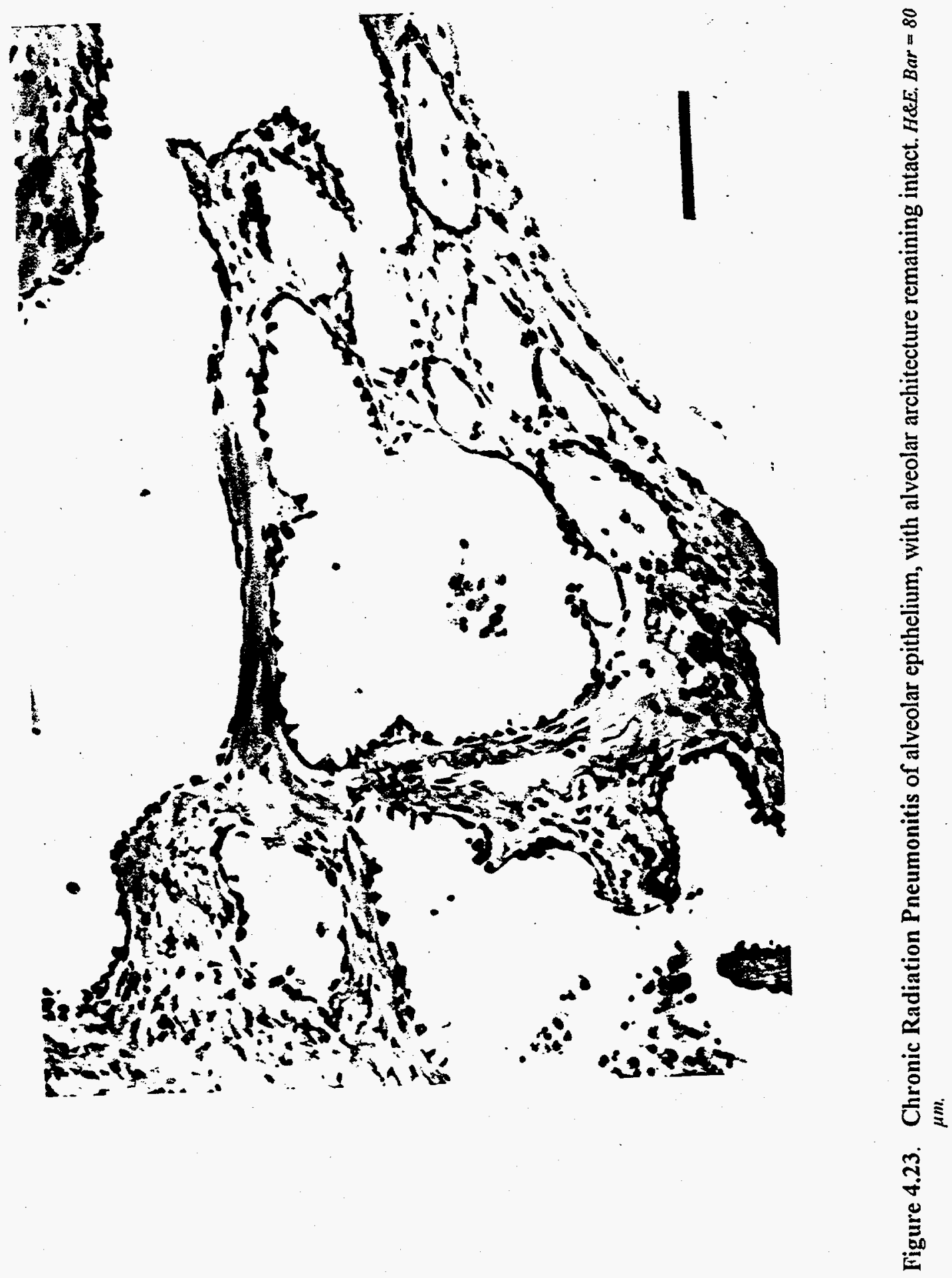


Thyroid 


\title{
CHAPTER 5: Thyroid Tumors
}

\author{
Patrick J. Haley \\ Inhalation Toxicology Research Institute \\ (Currently at Nycomed Inc.) \\ Stephen A. Benjamin \\ Colorado State University
}

\section{INTRODUCTION}

Because beagles have been reported to be at increased risk over other breeds of dogs for the development of spontaneous thyroid neoplasms, it is important to establish the histological characteristics of thyroid tumors in control populations of this breed. The beagle colonies at the Department Of Energy/Office of Health and Environmental Research contract laboratories afford a unique opportunity to study spontaneous thyroid neoplasms in populations of normal dogs with lifetime health histories and to determine the effect of various carcinogens on the thyroid gland. This chapter should be considered an extenstion of earlier work reported by: Brodey and Kelley 1968, Van Sandersleben and Hanichem 1974, Hayes and Fraumeni 1975, Leav et al. 1976, Mitchell, Hurov and Troy 1979, Birchard and Roesel 1981, and Harari, Patterson, and Rosenthal 1986.

An outline of principal lesions observed in beagle dogs, with an index to illustrations and SNOMED database codes, is shown in Table 5.1.

\section{DESCRIPTIONS}

\section{Follicular Hyperplasia}

Nodular hyperplasia is a frequently encountered incidental lesion of old dogs. These lesions are usually nonfunctional. Nodular hyperplasia is characterized by multiple irregular foci of hyperplastic follicular cells that are sharply delineated but not encapsulated from adjacent tissue. Histologic characteristics are variable and include small follicles containing little or no colloid, or hyperplastic columnar cells lining large, dilated, cystic spaces with abundant, deeply staining colloid present (Figure 5.1). Compression of adjacent thyroid parenchyma is not usually observed.

\section{Idiopathic Follicular Atrophy}

In idiopathic follicular atrophy, progressive loss of degenerating follicular epithelium is accompanied by interstitial infiltration by adipose cells, resulting in the isolation of small aggregates of active follicles. The appearance of increased interstitial connective tissue is more a product of stromal collapse and condensation than fibrosis. Persisting follicular epithelial cells may be hyperplastic, with tall columnar cells lining small follicles filled with deeply eosinophilic and/or vacuolated colloid (Figure 5.2). A small encapsulated adenoma or nonencapsulated area of nodular hyperplasia may be present also in affected glands. Inflammatory cells (lymphocytes and/or macrophages) are not usually present. 
Table 5.1. Thyroid Neoplasia nomenclature, index to figures, and SNODOG database codes

\begin{tabular}{|c|c|c|}
\hline Nomenclature & Figure(s) & $\begin{array}{c}\text { SNODOG } \\
\text { Morphology } \\
\text { code }\end{array}$ \\
\hline \multicolumn{3}{|l|}{ Non-neoplastic } \\
\hline Follicular hyperplasia & $5: 1$ & $\mathrm{M} 72000 \mathrm{~F}$ \\
\hline Idiopathic follicular atrophy & 5.2 & M580005 \\
\hline Lymphocytic thyroiditis & 5.3 & M430000 \\
\hline \multicolumn{3}{|l|}{ Benign Tumors } \\
\hline Follicular adenoma & 5.4 & $\mathrm{M} 833000$ \\
\hline Other non-neoplastic lesions & $5.5-8$ & \\
\hline \multicolumn{3}{|l|}{ Malignant } \\
\hline Follicular carcinoma & 5.9 & M833030 \\
\hline Solid carcinoma & $5.10-12$ & $M 823030$ \\
\hline Solid follicular carcinoma & $5.13-17$ & $\mathrm{M} 823033$ \\
\hline Oxyphil tumor & $5.18-19$ & M829030 \\
\hline Papillary carcinoma & & $M 805030$ \\
\hline Squamous cell carcinoma & & $\mathrm{M} 807030$ \\
\hline Anaplastic carcinoma & & M802030 \\
\hline C-cell carcinoma & & M851030 \\
\hline Ectopic thyroid tumor & . & $b$ \\
\hline Other neoplastic lesions & . & \\
\hline
\end{tabular}

\section{Lymphocytic Thyroiditis}

Spontaneous lymphocytic thyroiditis is an autoimmune disease with a genetic basis that occurs frequently in some beagle colonies. Lymphocytic thyroiditis is characterized by multifocal to diffuse infiltration of the thyroid by lymphocytes, plasma cells, and macrophages that penetrate between follicle-lining cells and accumulate with follicular lumens, along with degenerative epithelial cells. Nodular lymphoid accumulation with the thyroid may be observed, accompanied by follicular degeneration and interstitial fibrosis (Figure 5.3). Concomitant C-cell hyperplasia may be noted.

\section{Follicular Adenoma}

Grossly, follicular adenomas appear as small, well-demarcated, solid, tan-to-dark brown nodules. Follicular tumors are considered benign upon histologic examination when they are encapsulated by a fibrous connective tissue capsule, compress surrounding tissue, and display uniform cellular characteristics (Figure 5.4). The capsule may be thick and surround the nodule entirely, or thin and incomplete but without extension of the mass into adjacent tissue. Variably thick, fibrous connective tissue bands may arise from the capsule and penetrate irregularly into the mass. Follicular tumors are typically microfollicular with some areas of solid cord development. They may be singular or multiple, making differentiation from nodular hyperplasia difficult. Neoplastic cells have uniformly rounded and slightly vesiculate nuclei with single nucleoli. Cells are cuboidal to polygonal and contain ample granular eosinophilic to basophilic cytoplasm. In some areas, the cytoplasm may be 
markedly vacuolated. The cells may be arranged in small aggregates or narrow twisting cords, two to four cells thick, surrounded by a thin fibrovascular stroma. Mitotic figures are not usually apparent.

\section{Other Non-Neoplastic Lesions}

There is as yet no prognostic significance for subdivisions of benign tumors such as papillary, cystic, solid, or combinations thereof. Tumors with a solid follicular pattern are usually grouped under the broader title of follicular adenomas (Figures 5.5 and 5.6). Cystic adenomas are welldelineated, tan-to-dark brown nodules, with a cystic center filled with serosanguinous fluid (Figures 5.7 and 5.8). The wall of the cyst may be fibrous and lined with single to multiple layers of cuboidal to tall columnar epithelial cells and variably sized, arborizing papillary fronds projecting into the cyst lumen. Solid papillary adenomas are characterized by cuboidal to columnar epithelial cells resting on linear and branching papillary fronds without definite cyst formation.

\section{Follicular Carcinoma}

Follicular carcinomas are characterized by a predominance of follicular differentiation with cells forming both macrofollicular and microfollicular patterns that are accompanied occasionally by tubular and papilliferous areas (Figure 5.9). The size of follicles may be constant or may vary considerably throughout the tumor, with larger follicles becoming irregular and linear in some areas. Follicular lining cells may be cuboidal to tall columnar with basally located, vesicular nuclei and ample eosinophilic cytoplasm. Staining may be more basophilic in those tumors with decreased amounts of cytoplasm. Production of colloid is variable and, typically, most intense within larger follicles; small follicles and acinar structures may be devoid of colloid. While capsular and vascular invasion occurs often, cellular pleomorphism and atypia do not. Stromal development is variable.

\section{Solid Carcinoma}

Solid thyroid carcinomas are characterized by large numbers of neoplastic epithelial cells arranged in densely cellular sheets, thick multicellular cords, and/or small to large irregularly shaped aggregates separated by a variably thick fibrovascular stroma (Figures 5.10, 5.11, and 5.12). Cords of cells, which vary from 2 to 20 or more cells thick, may occur in linear twisting patterns that occasionally suggest tubular and papillary structures. The fibrovascular stroma may progress from very thin strands that separate small aggregates of epithelial cells into micronodules, to thick bands coursing irregularly throughout the mass, breaking it up into larger discrete nodules. In some tumors, the stroma may be quite delicate and barely discernible against the background of solid sheets of neoplastic cells. Severe, centrally located necrosis and hemorrhage are frequently present in some tumors.

The neoplastic epithelial cells are typically large, cuboidal to polygonal, with round to ovoid and vesicular nuclei and singular nucleoli. Cytoplasm is ample, eosinophilic, and granular in most cases; however, some tumors may have cells with a small amount of granular and basophilic to gray cytoplasm. Cellular pleomorphism is not typically prominent but may occur in highly malignant variants, along with increased numbers of mitotic figures. Aggregates of oxyphilic cells may be present and trapped within the background of neoplastic cells.

Capsular invasion and/or vascular penetration are usual in these tumors and form the basis for a diagnosis of malignancy.

\section{Solid Follicular Carcinoma}

Solid follicular carcinomas display characteristics previously described for both follicular carcinomas and solid carcinomas, with both components being present in approximately equal ratios (Figures 5.13 through 5.17). The follicular component may be of either the micro- or macrofollicular 


\section{Thyroid}

pattern and scattered throughout the tumor, or it may alternate with entirely solid regions. As noted previously, nuclear and cellular pleomorphism is infrequent, while capsular and vascular invasion often occurs.

\section{Oxyphil Tumor}

A variant of the solid carcinoma is the oxyphil tumor, which is characterized by a somewhat uniform population of polygonal, strongly cosinophilic cells arranged in solid sheets without follicular development (Figures 5.18 and 5.19). Nuclei are slightly pleomorphic, vesiculate, irregularly rounded, and usually located eccentrically within homogenous eosinophilic cytoplasm. While there is extensive penetration by a fine fibrovascular stroma, the neoplastic cells show no specific orientation and may tend to pile up in some areas. Penetration of the capsule and/or vasculature attests to the malignancy of this tumor.

\section{Papillary Carcinoma}

Papillary carcinomas, while reported frequently in humans, are rare in dogs and have not been observed in the dogs examined here. Arborizing fibrovascular stalks and papillary fronds predominate, accompanied by a marked follicular pattern.

\section{Squamous Cell Carcinoma}

Squamous cell carcinomas of the thyroid have been described in dogs but have not been identified in the beagles considered here. Histologic criteria are the same for squamous cell carcinomas, arising from any other epithelial surface and include keratinizing cells with intercellular bridges. Squamous cell carcinomas arising in the tonsil and metastasizing to the thyroid have been observed. The possibility of such a phenomenon should be carefully evaluated whenever a squamous cell carcinoma is observed in the thyroid.

\section{Anaplastic Carcinoma}

Anaplastic or undifferentiated carcinomas of the thyroid have been described in dogs but have not been identified in the beagles considered here. The tumors are typically solid, with a marked degree of cellular cell formation.

\section{C-Cell Carcinoma}

C-cell carcinomas are reported occasionally in the dog but have not been identified in the beagles considered here. These tumors are usually solid, with either large or small polygonal cells that may resemble plasma cells. Alternatively, the cells may be columnar and separated into lobules by a fibrovascular stroma. The cells may be lined up perpendicularly to the basement membrane. A diagnostic aid is the presence of an amyloid-containing stroma; however, its occurrence is not invariable for this tumor. Immunoperoxidase techniques used for staining for the presence of calcitonin are a more reliable indicator of the parafollicular origin of these cells.

\section{Ectopic Thyroid Tumors}

Neoplasms arising at the base of the heart in beagles have been diagnosed as ectopic thyroid carcinomas, based on ultrastructural characteristics. Cells of the primary tumor and its metastases are characterized as polyhedral, with lightly eosinophilic, finely granular, and, occasionally, vacuolated cytoplasm with scattered PAS-positive granules. Nuclei are variable in size, round to oval, and centrally located. Mitotic figures are present frequently. Ultrastructural characteristics used to 
diagnose tumors of thyroid origin include well-developed cytoplasmic organelles, variable numbers of large intracytoplasmic vacuoles, absence of membrane-bound dense granules, and distinctive tubular or rod-like structures in the cytoplasm.

\section{Other Neoplastic Lesions}

Thyroid tumors of mesenchymal origin, which include osteosarcoma, chondrosarcoma, carcinosarcoma, and malignant epithelial-mesenchymal tumor, co-existent type, are extremely rare and have not been observed in these beagle colonies. This does not preclude their appearance, and diagnosis depends on the salient features of differentiation.

\section{REFERENCES}

Birchard, S. J., and O. F. Roesel. 1981. Neoplasia of the thyroid gland in the dog: a retrospective study of 16 cases. J. Am. Anim. Hosp. Assoc. 17:369-372.

Brodey, R. S. and D. F. Kelley. 1968. Thyroid neoplasms in the dog, a clinicopathologic study of fifty-seven cases. Cancer 22: 06-416.

Harari, J., J. S. Patterson, and R. C. Rosenthal. 1986. Clinical and pathologic features of thyroid tumors in 26 dogs. J. Am. Vet. Med. Assoc. 10:1160-1163.

Hayes, H. M. and J. F. Fraumeni, Jr. 1975. Canine thyroid neoplasms: epidemiologic features. J. Natl. Cancer Inst. 55:931-934.

Leav, I., A. L. Schiller, A. Rijnberk, M. A. Legg, and P. J. Der Kinderen. 1976. Adenomas and carcinomas of the canine and feline thyroid. Am. J. Pathol. 83:61-122.

Mitchell M., L. I. Hurov, and G. C. Troy. 1979. Canine thyroid carcinomas: clinical occurrence, staging by means of scintiscans and therapy of 15 cases. Vet. Surg. 8:112-118.

Van Sandersleben, J. and T. Hanichem. 1974. Chapter III, Tumors of the Thyroid Gland, pp. 3542. In: The Bulletin of the World Health Organization, International Histological Classification of Tumors of Domestic Animals, World Health Organization, Geneva, Switzerland. 
Thyroid

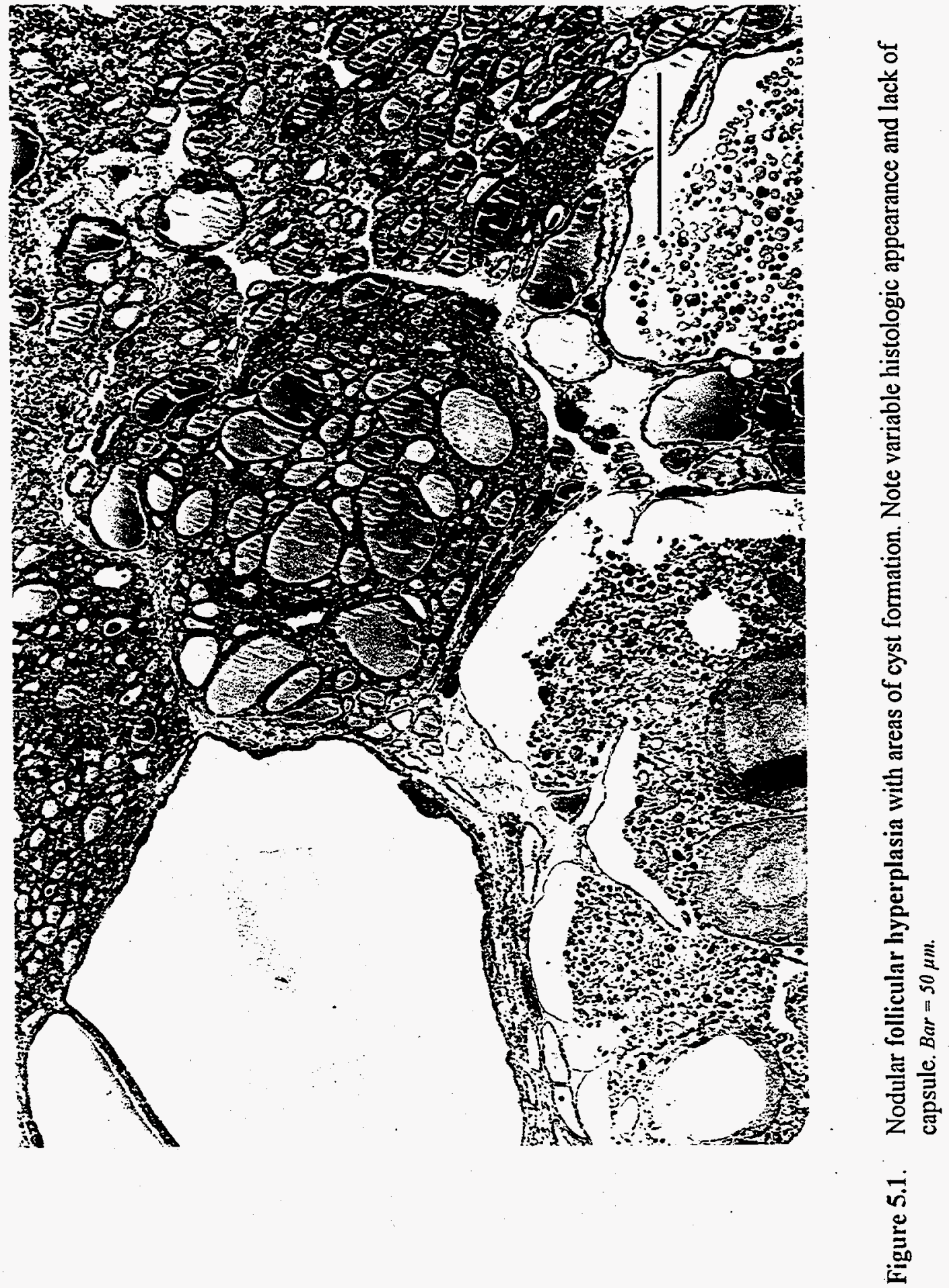




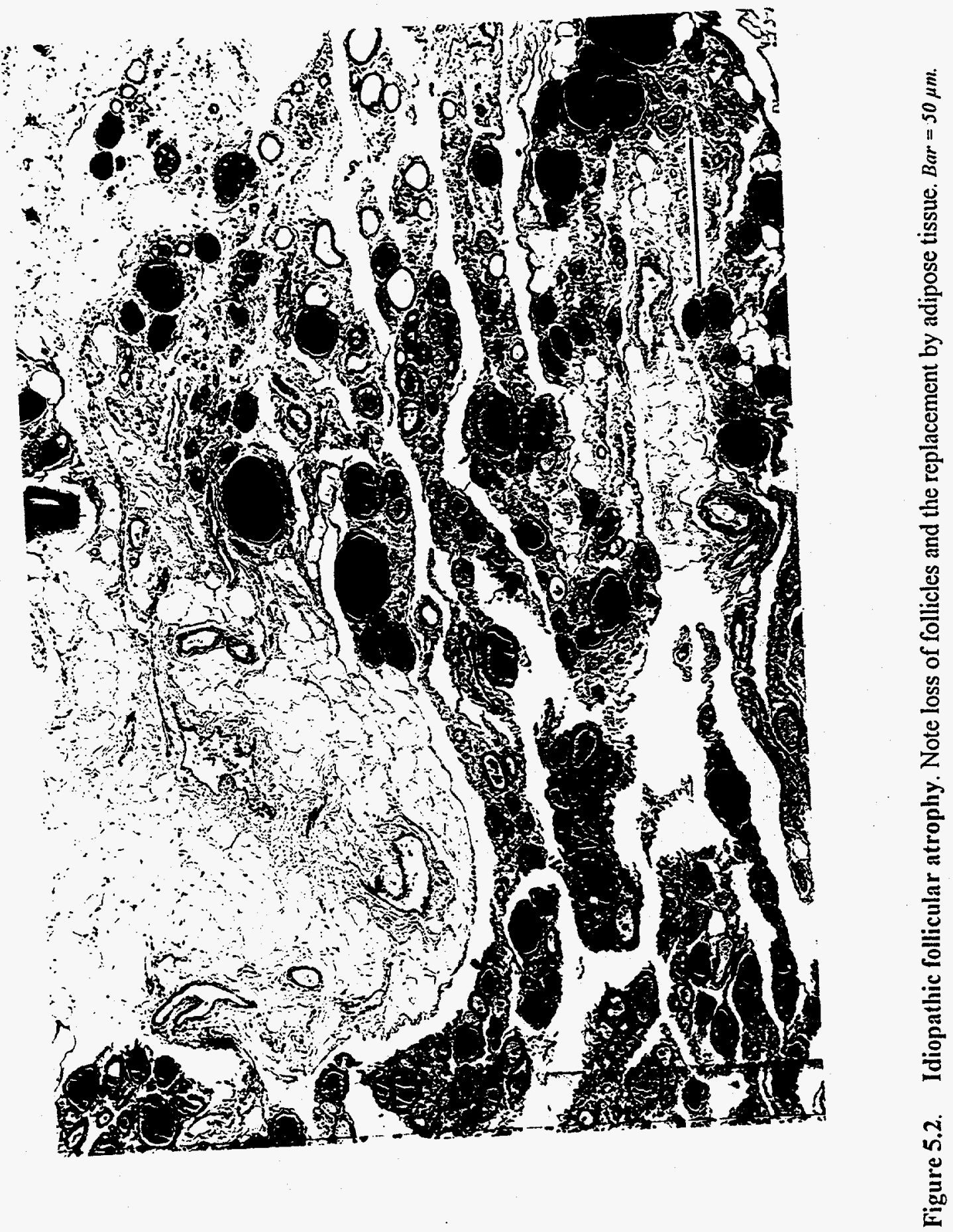




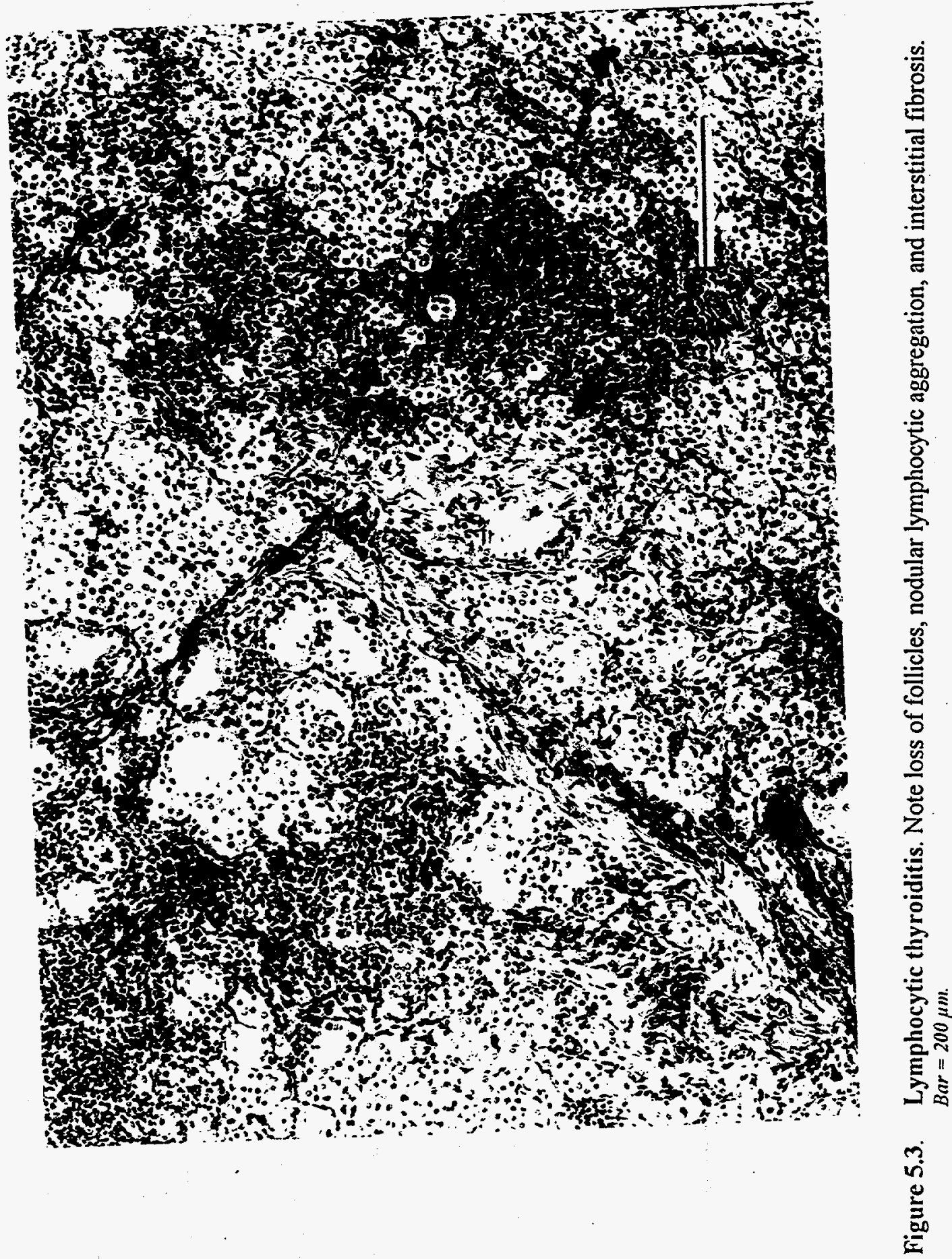


Thyroid

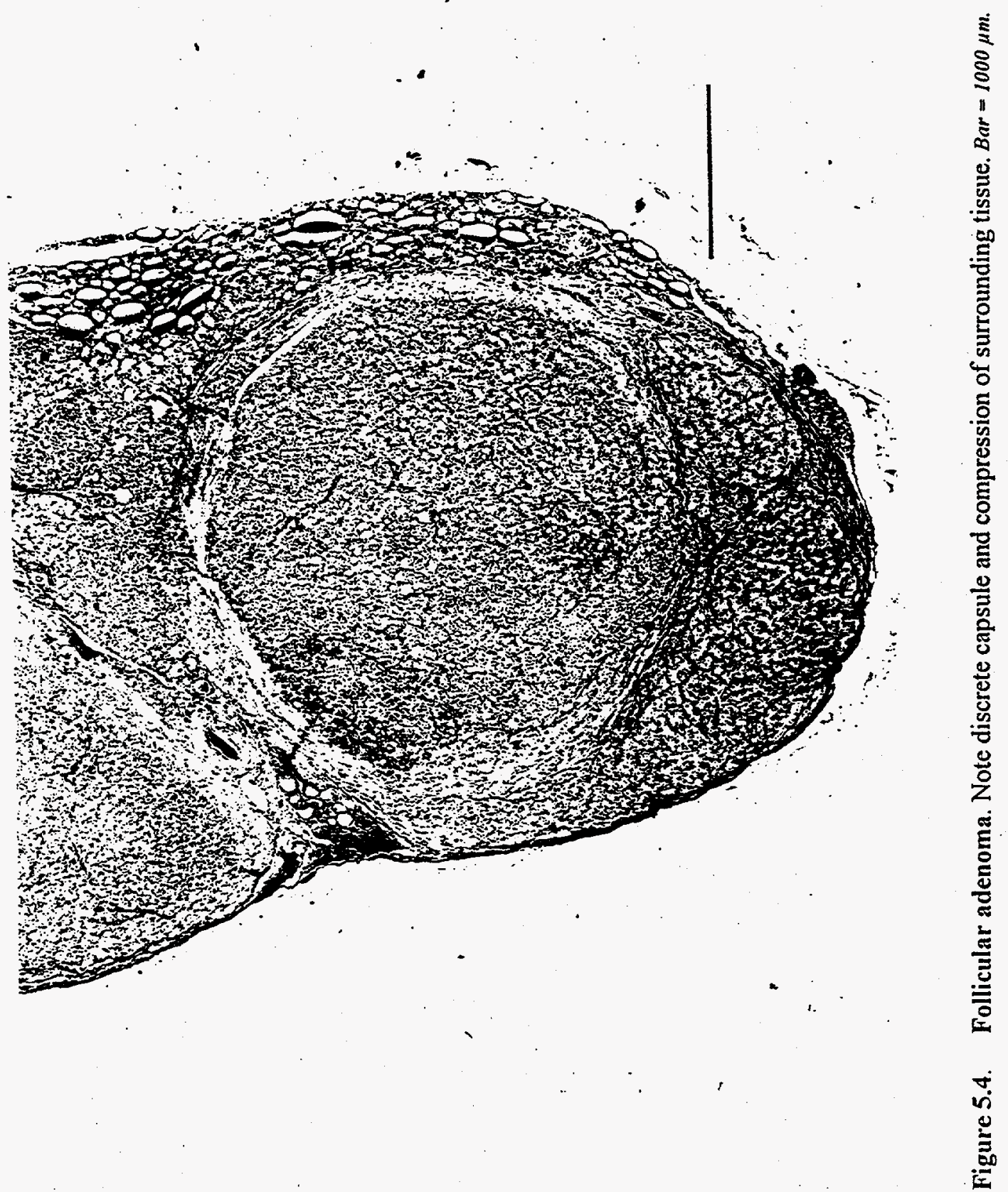


Thyroid

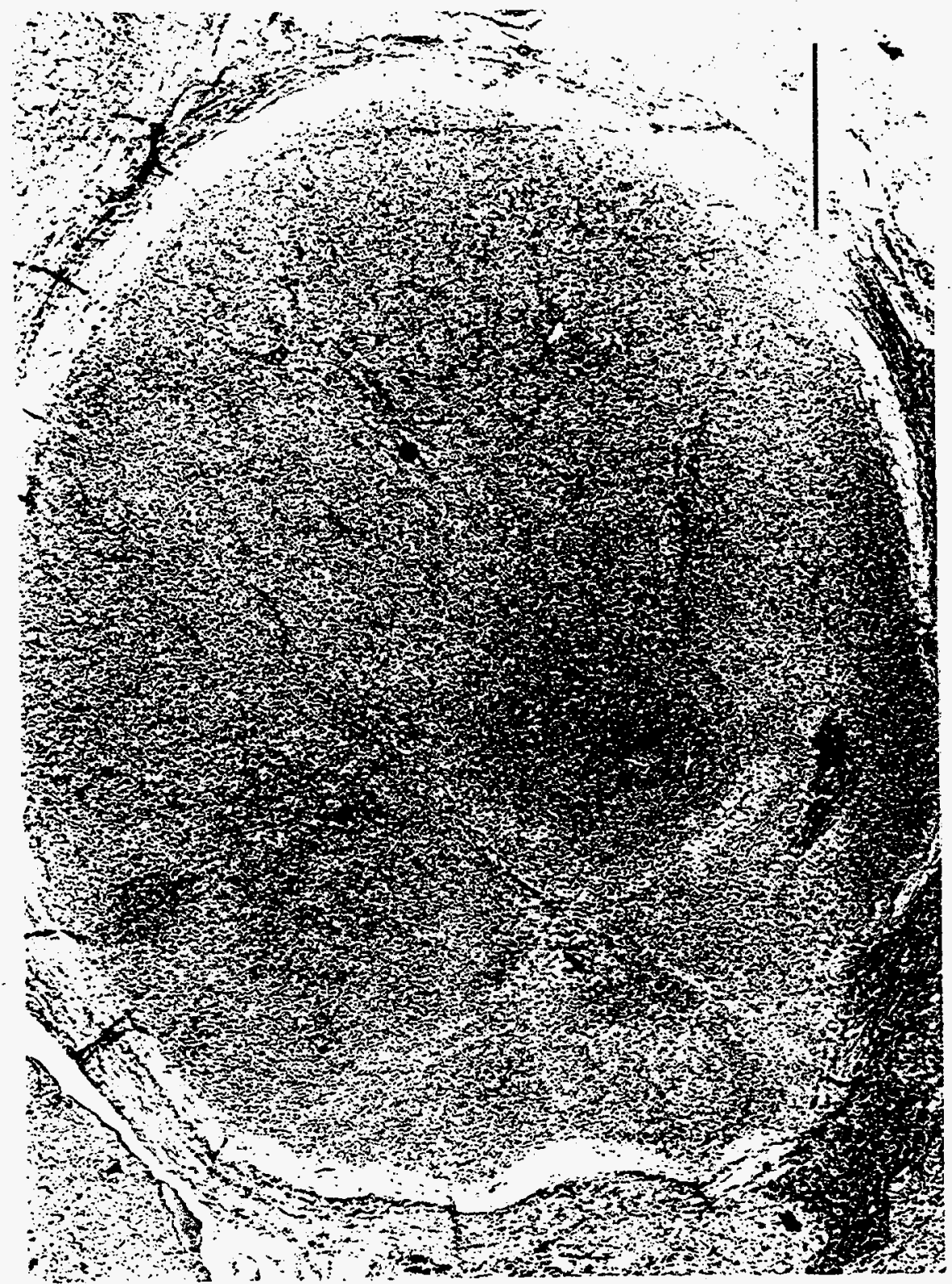

Oे 
Thyroid

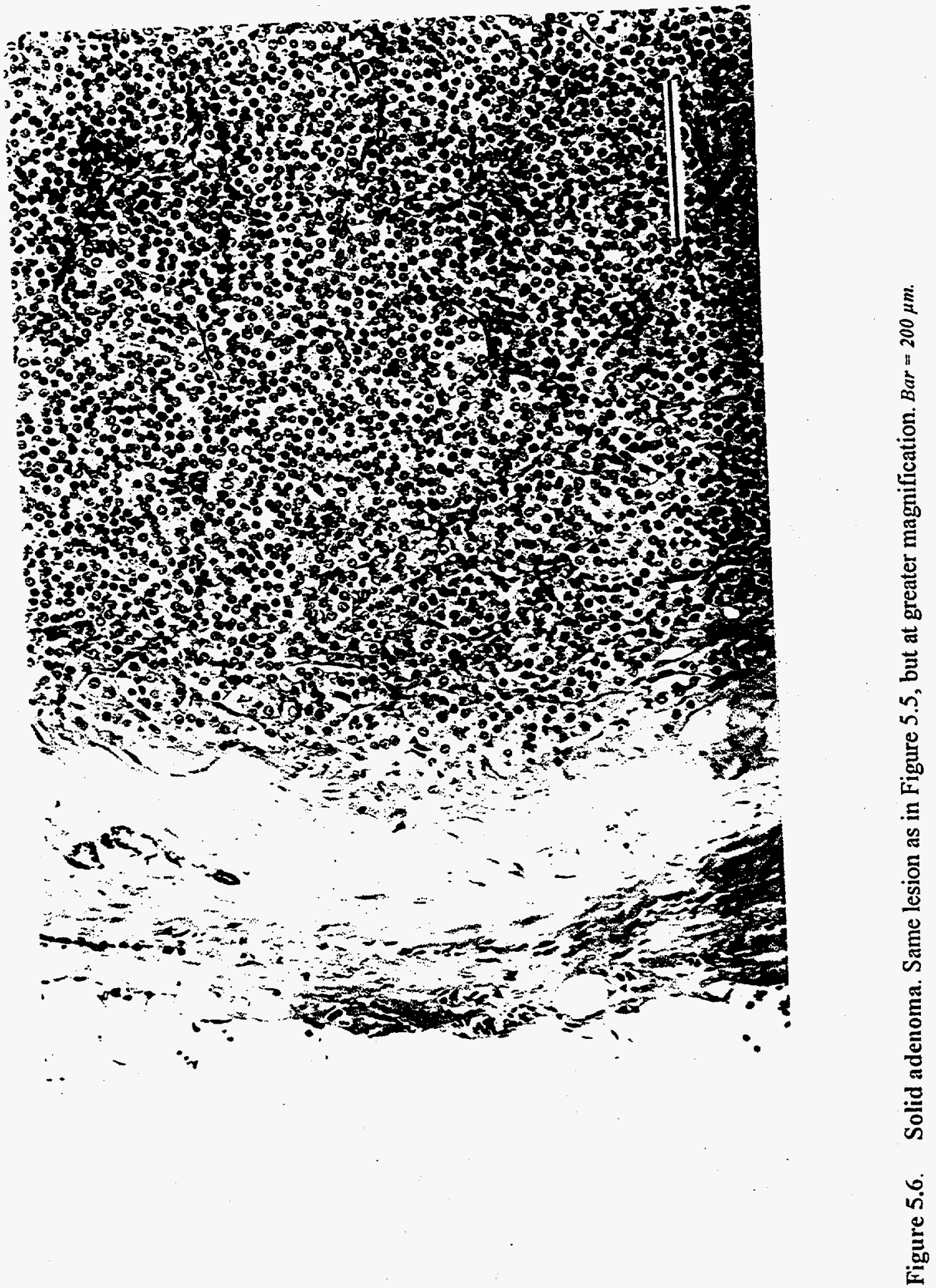


Thyroid

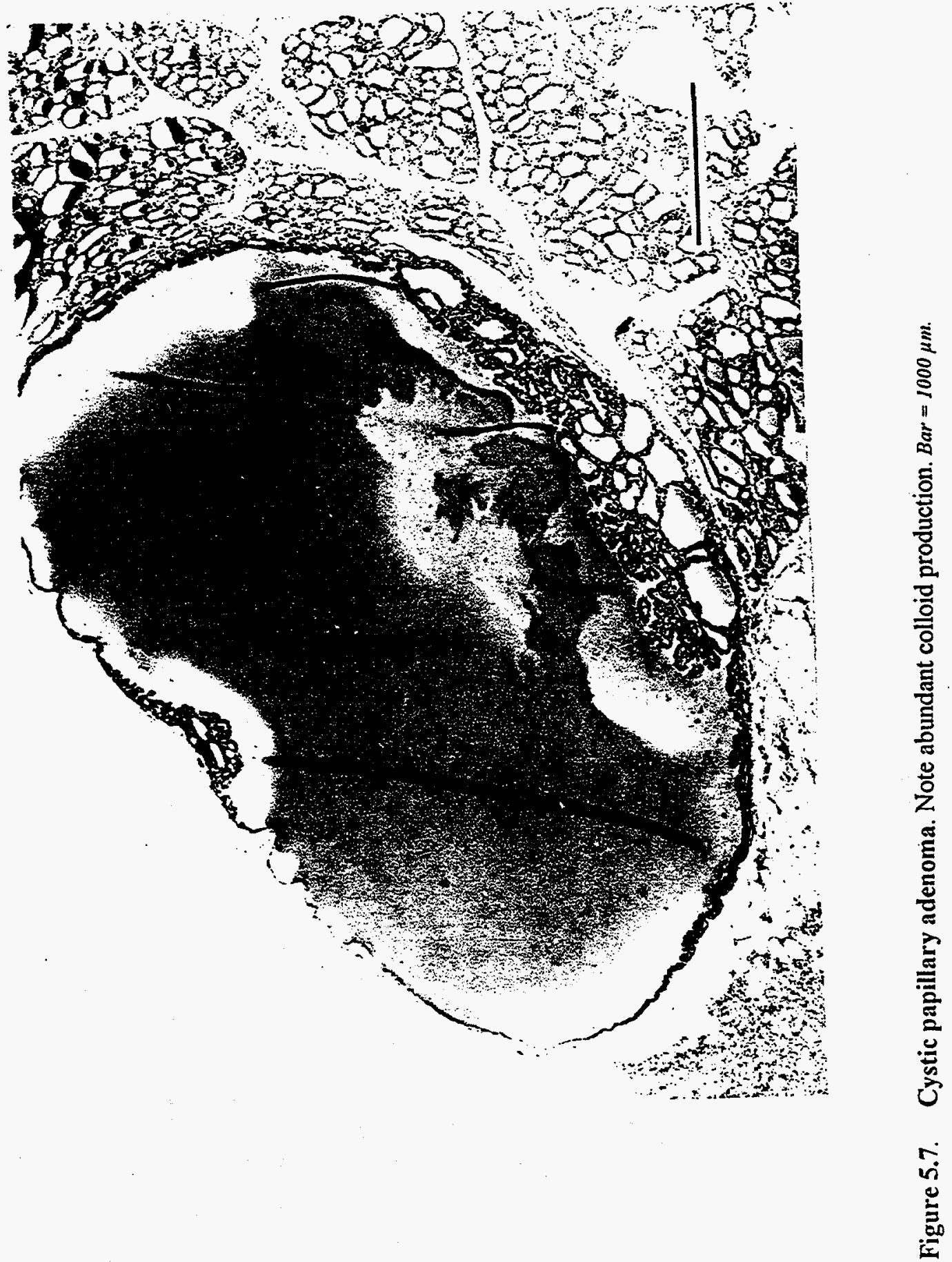




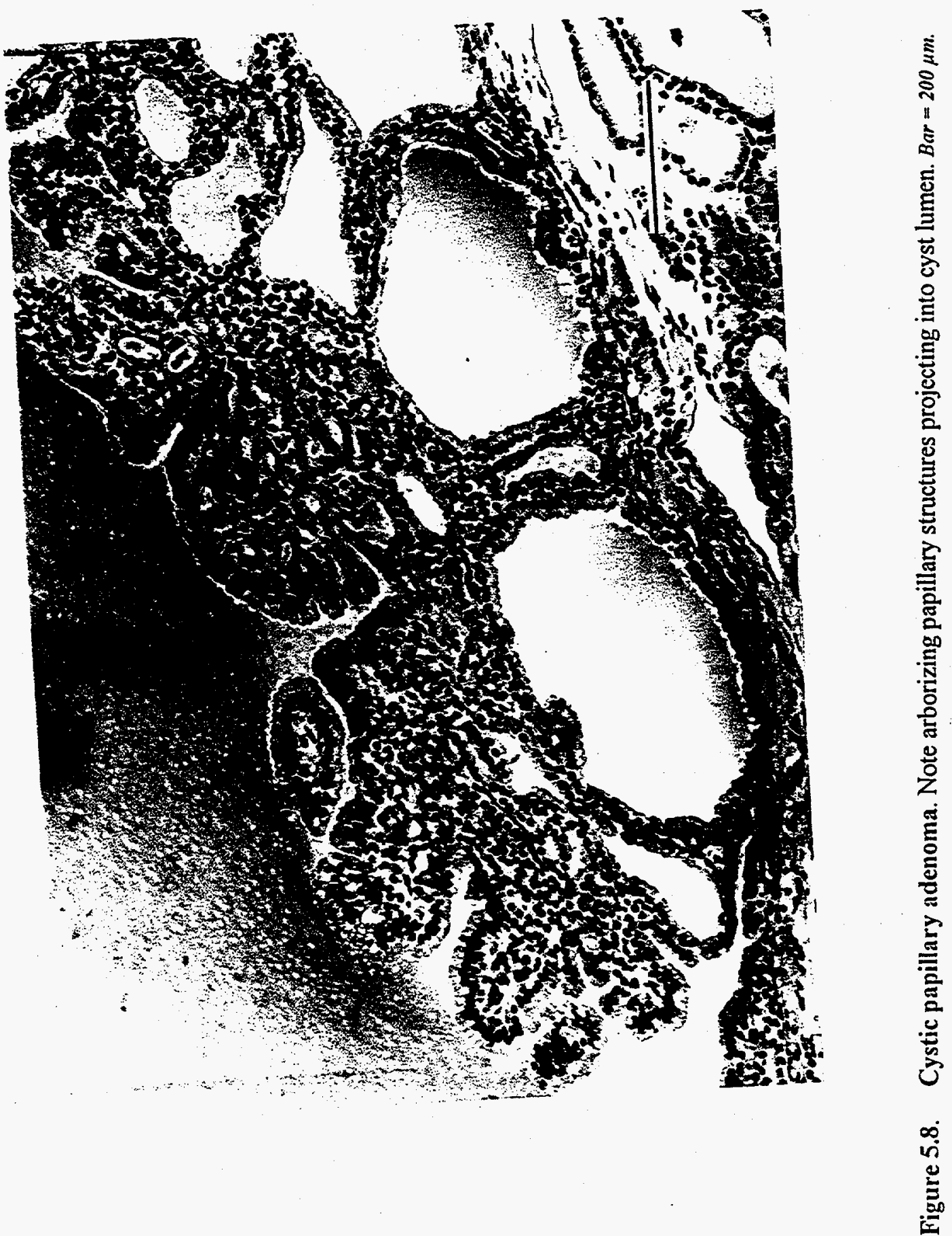




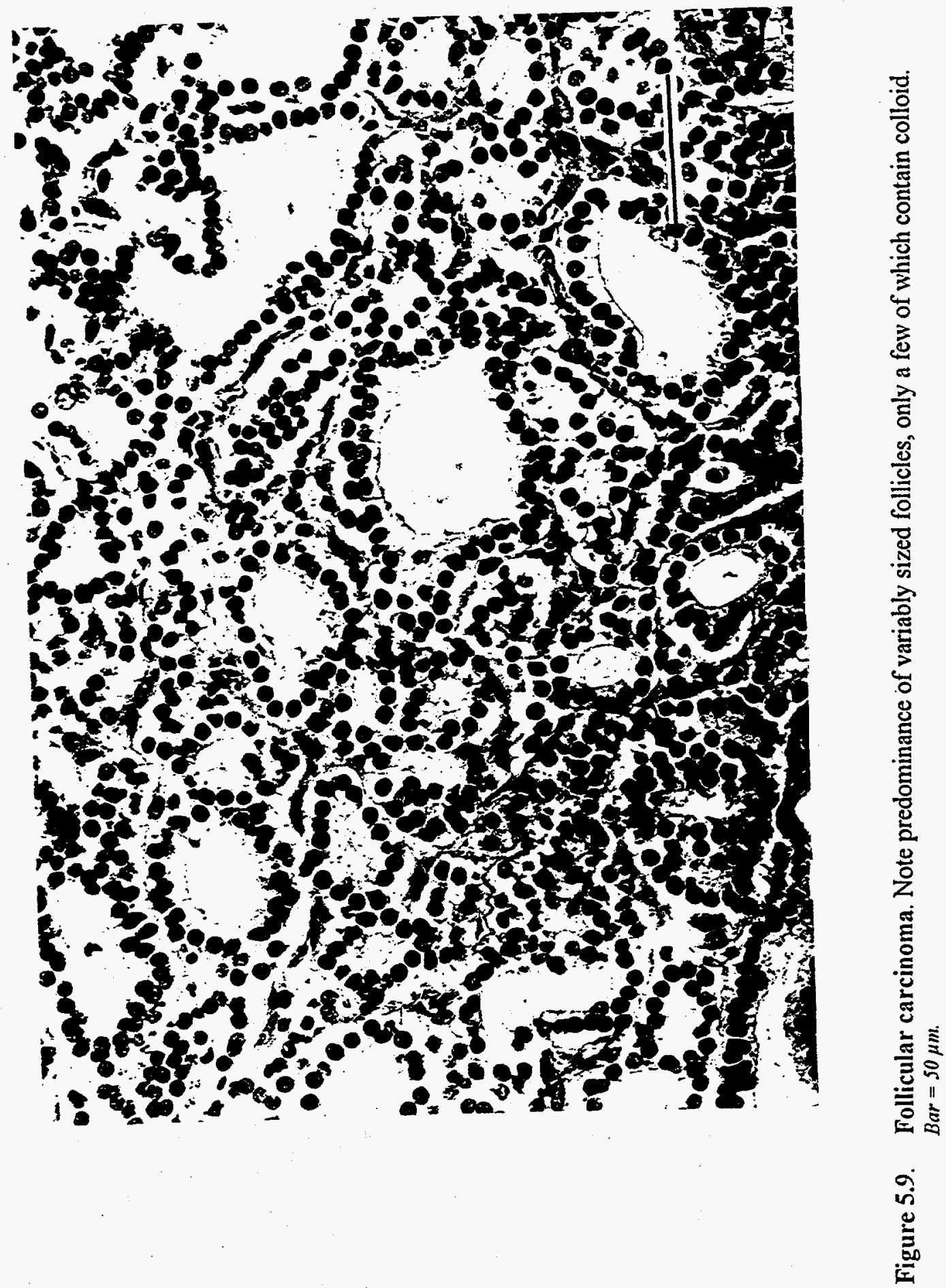


Thyroid

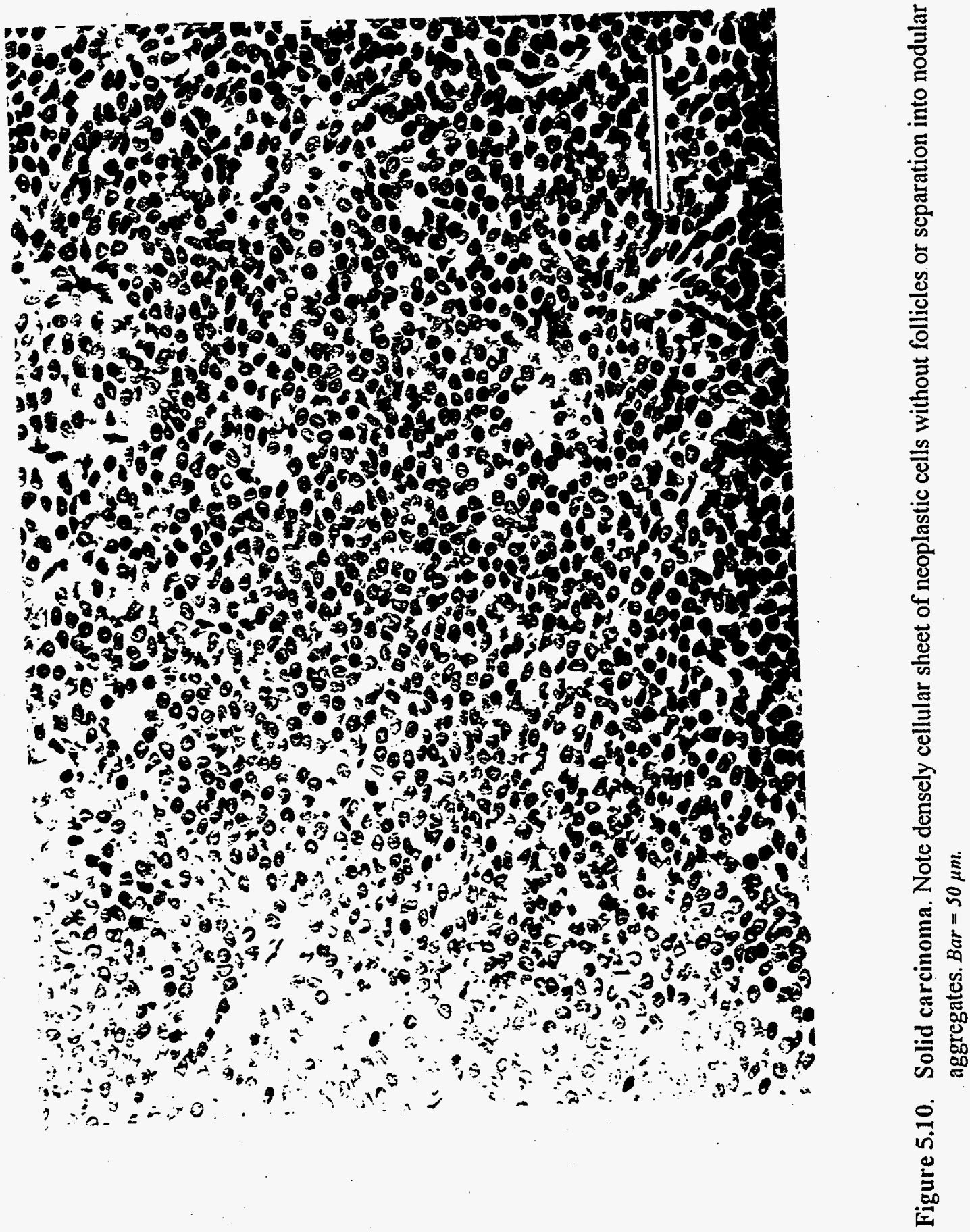


Thyroid

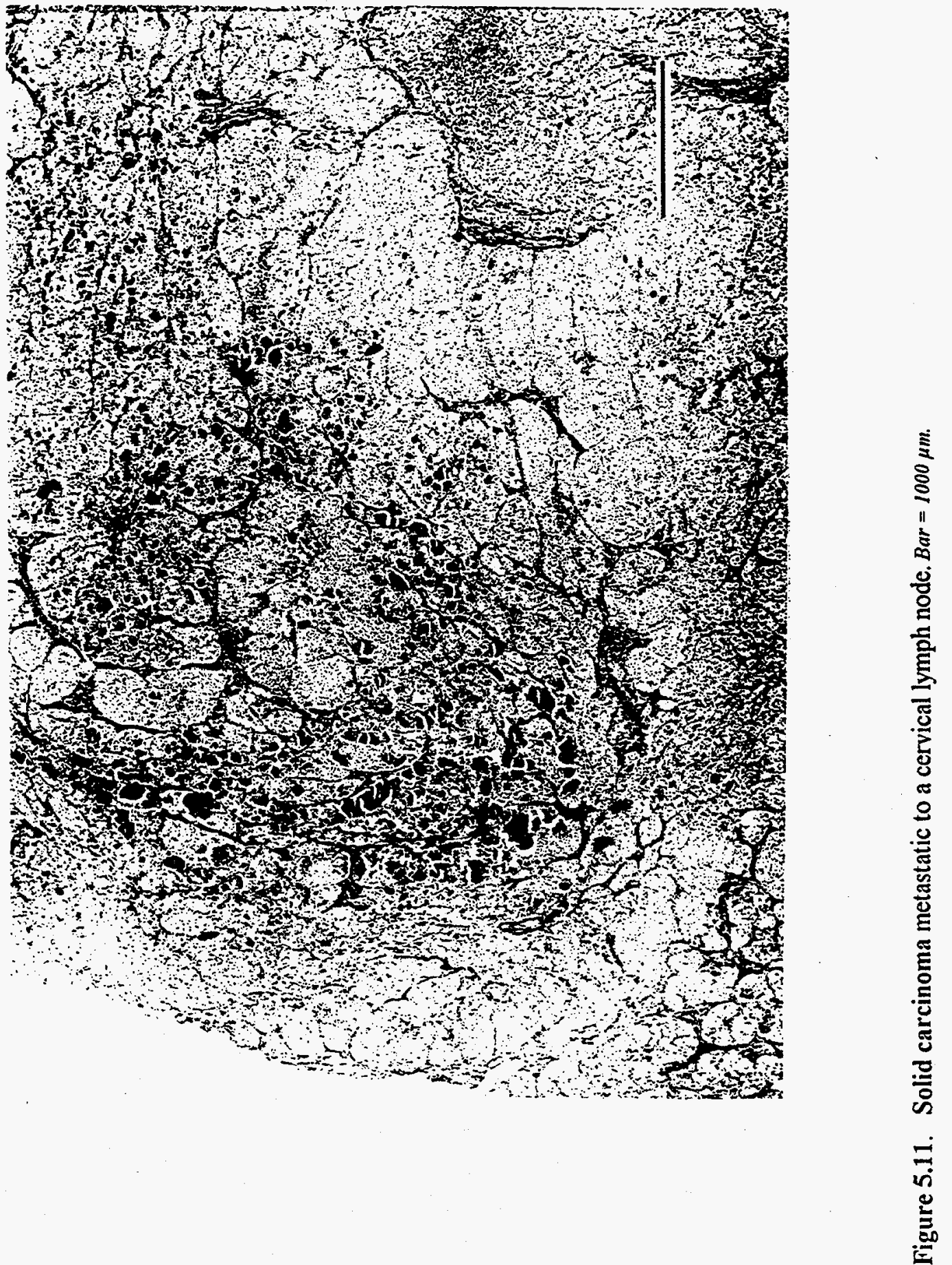


Thyroid

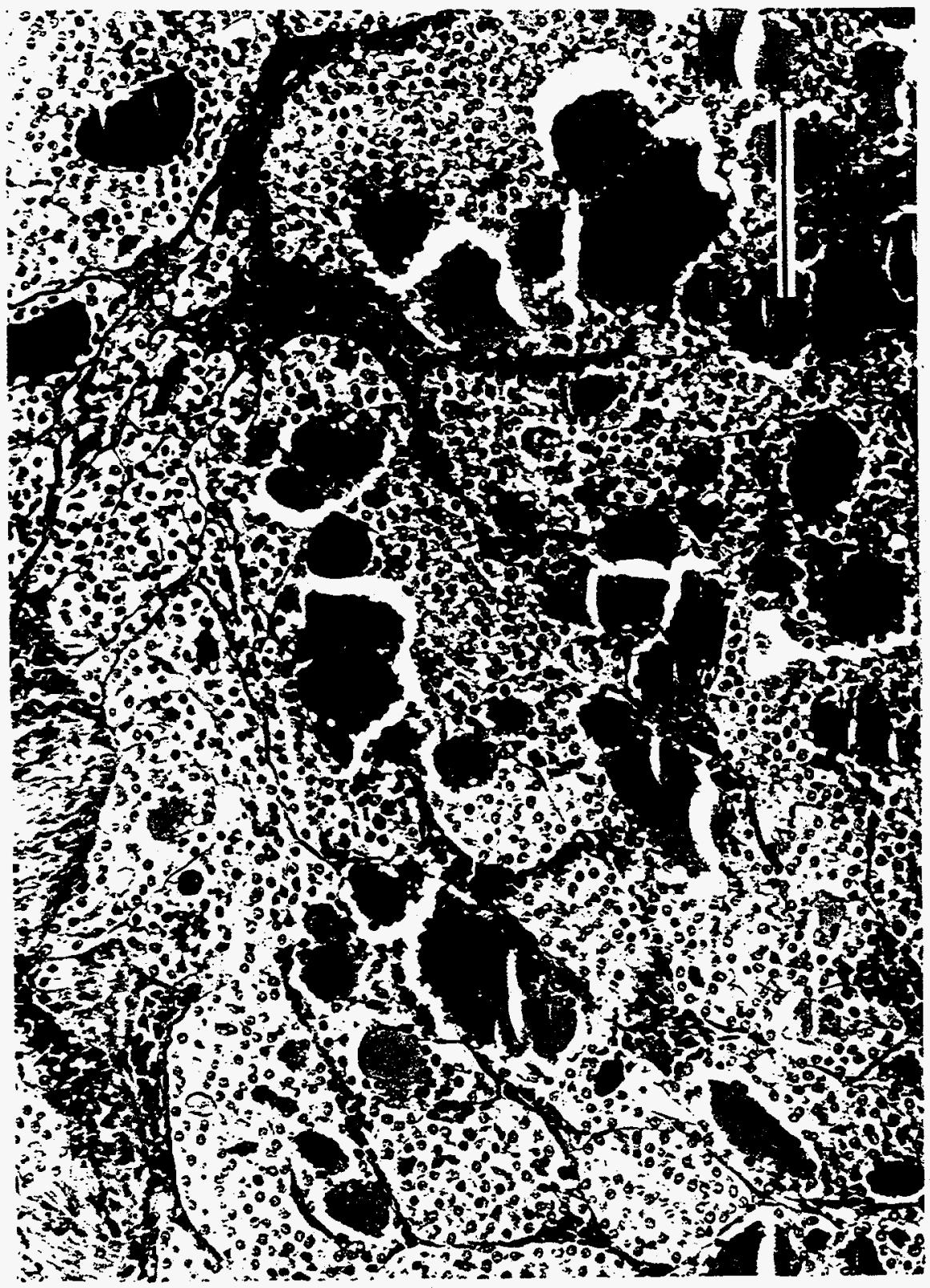

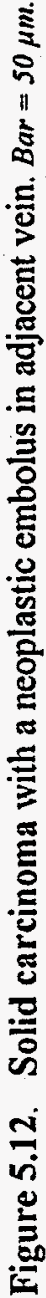




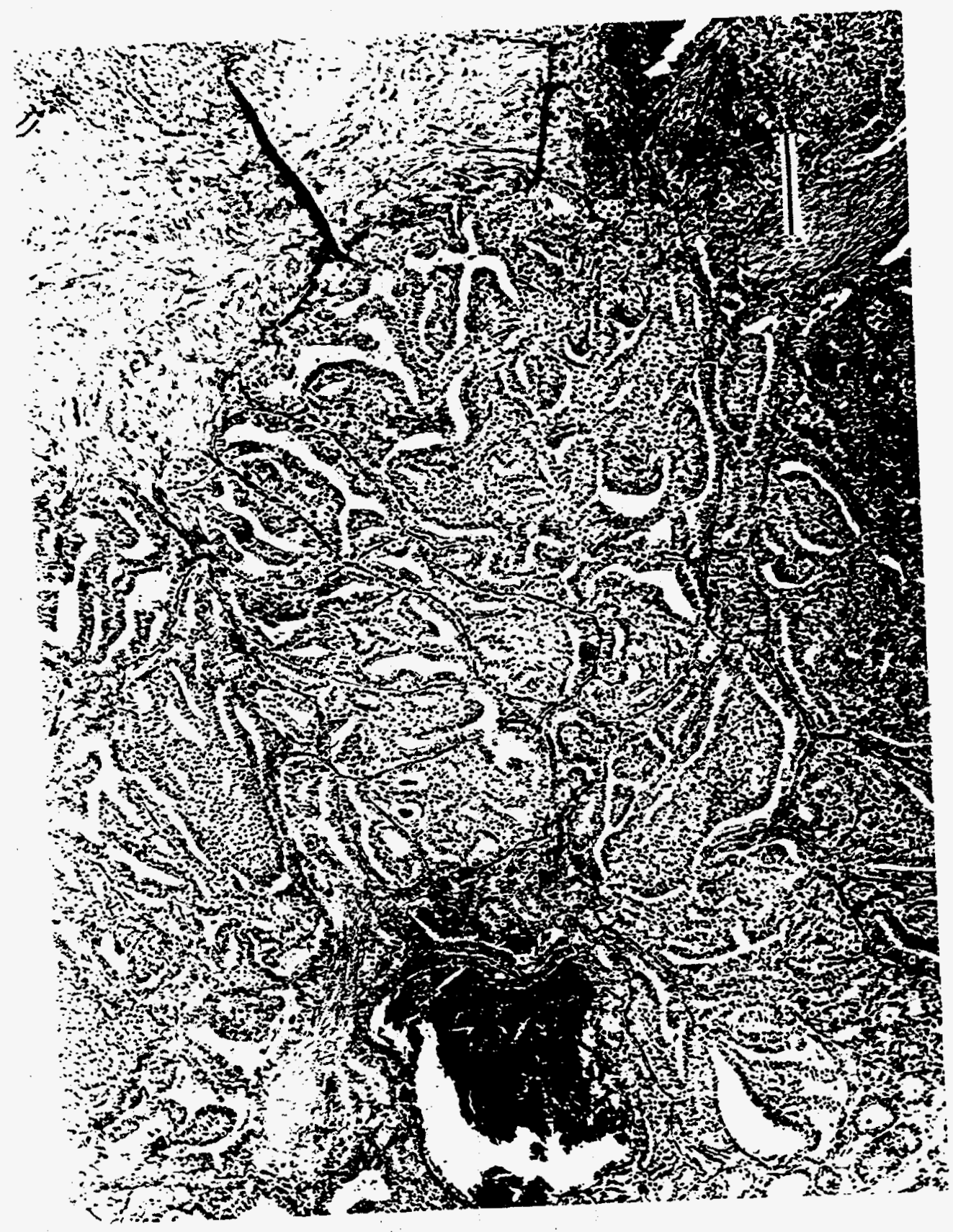

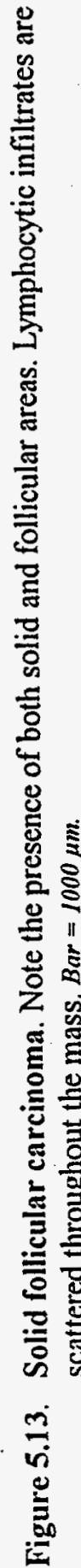




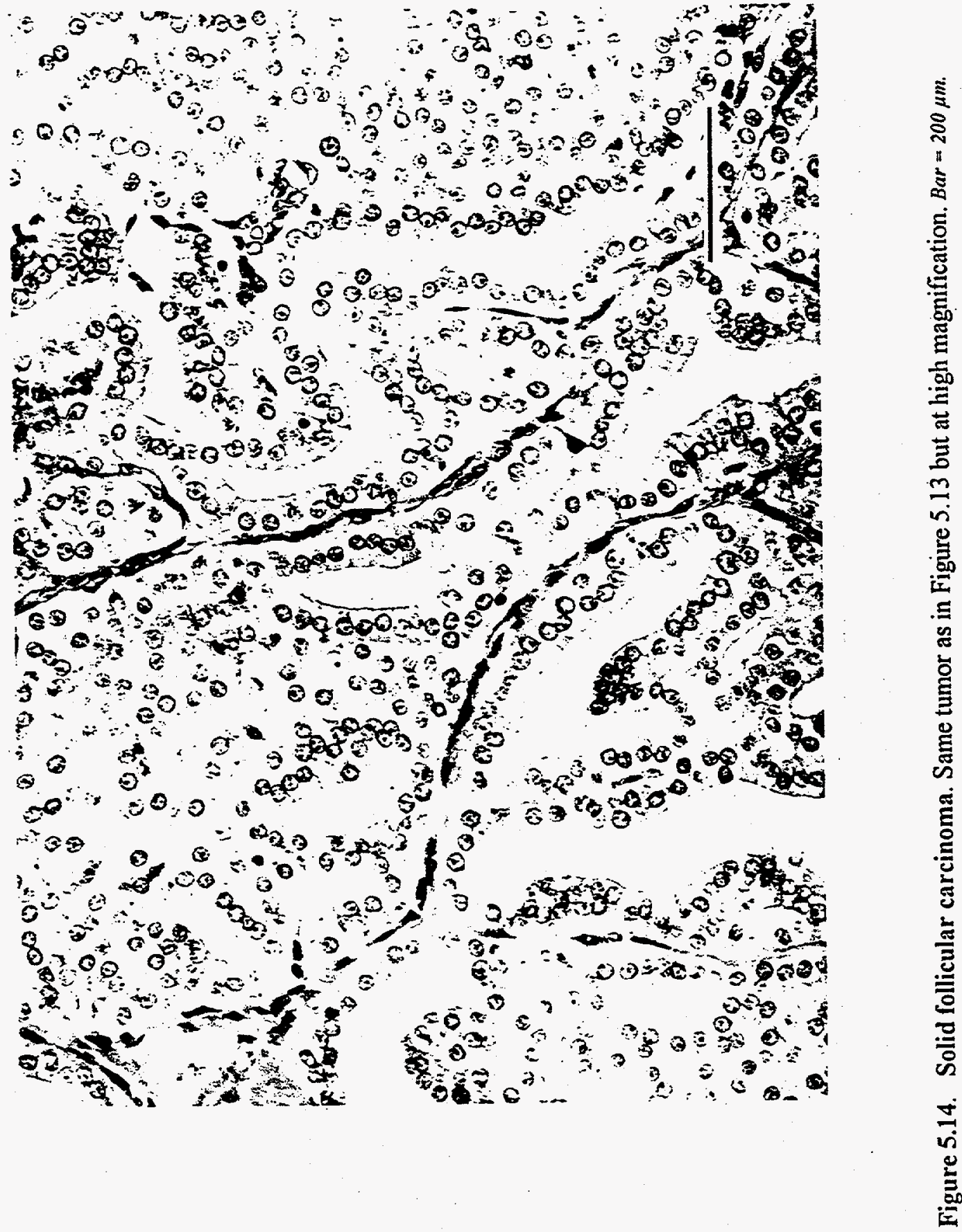


Thyroid

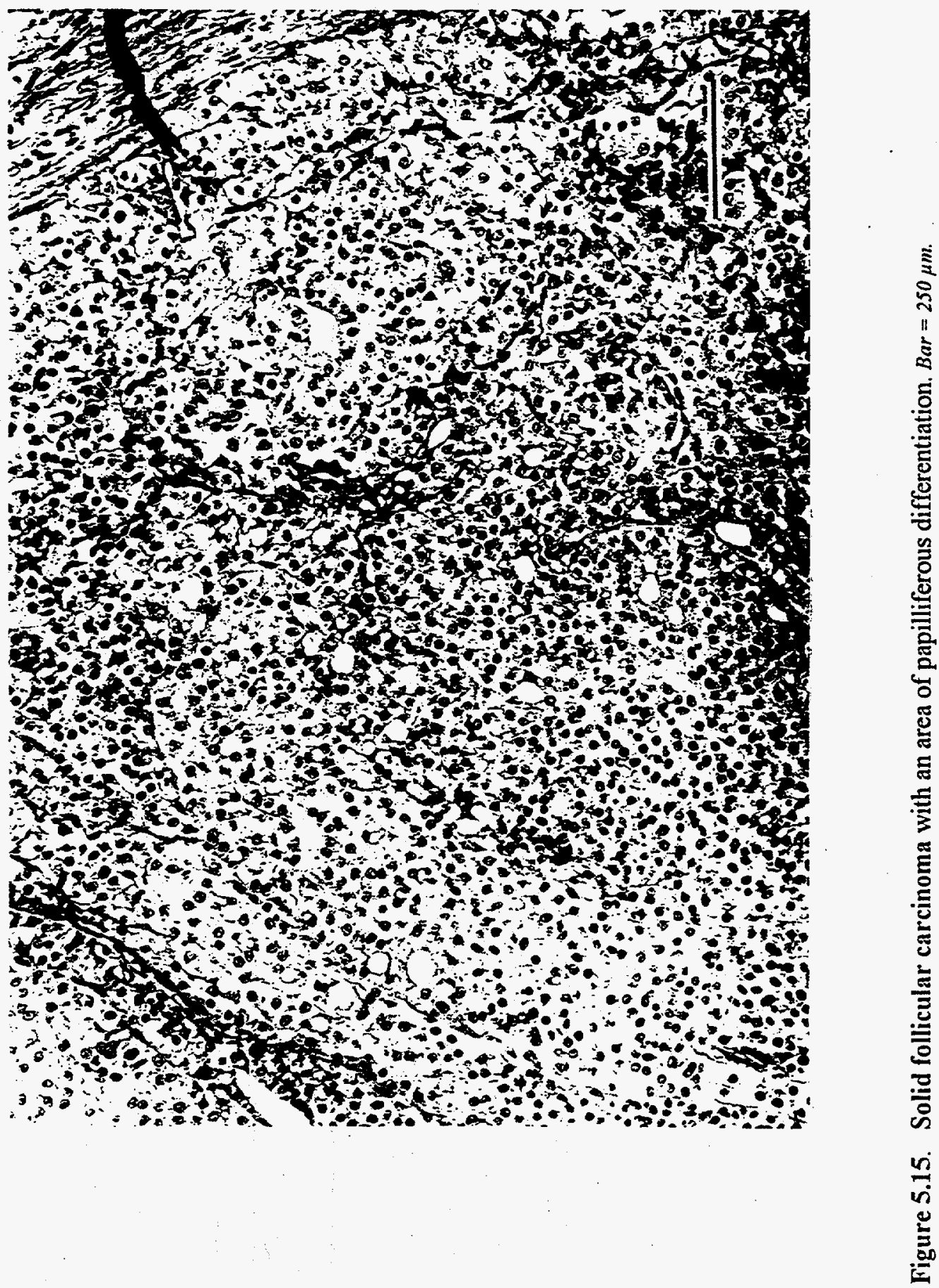


Thyroid

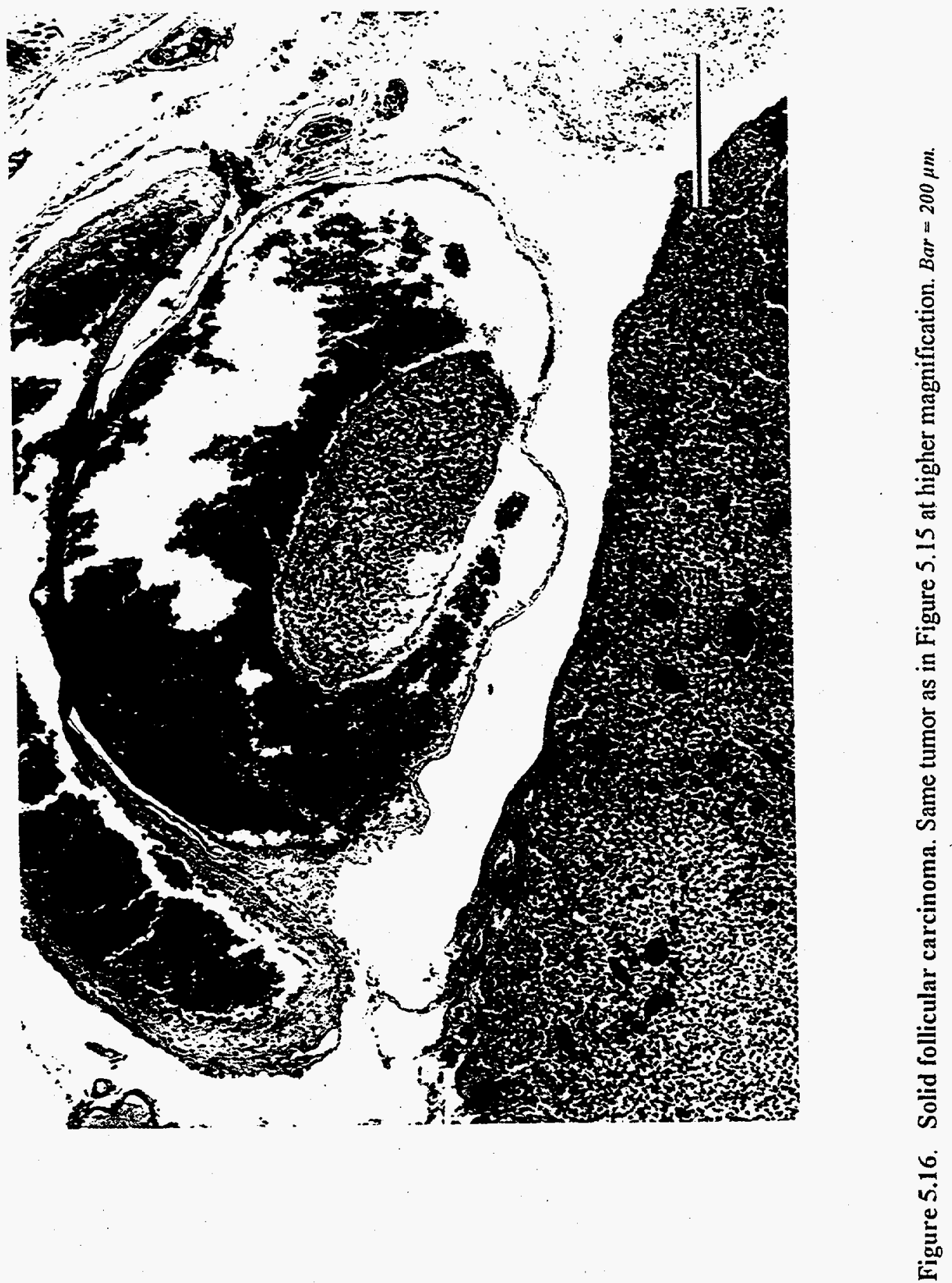




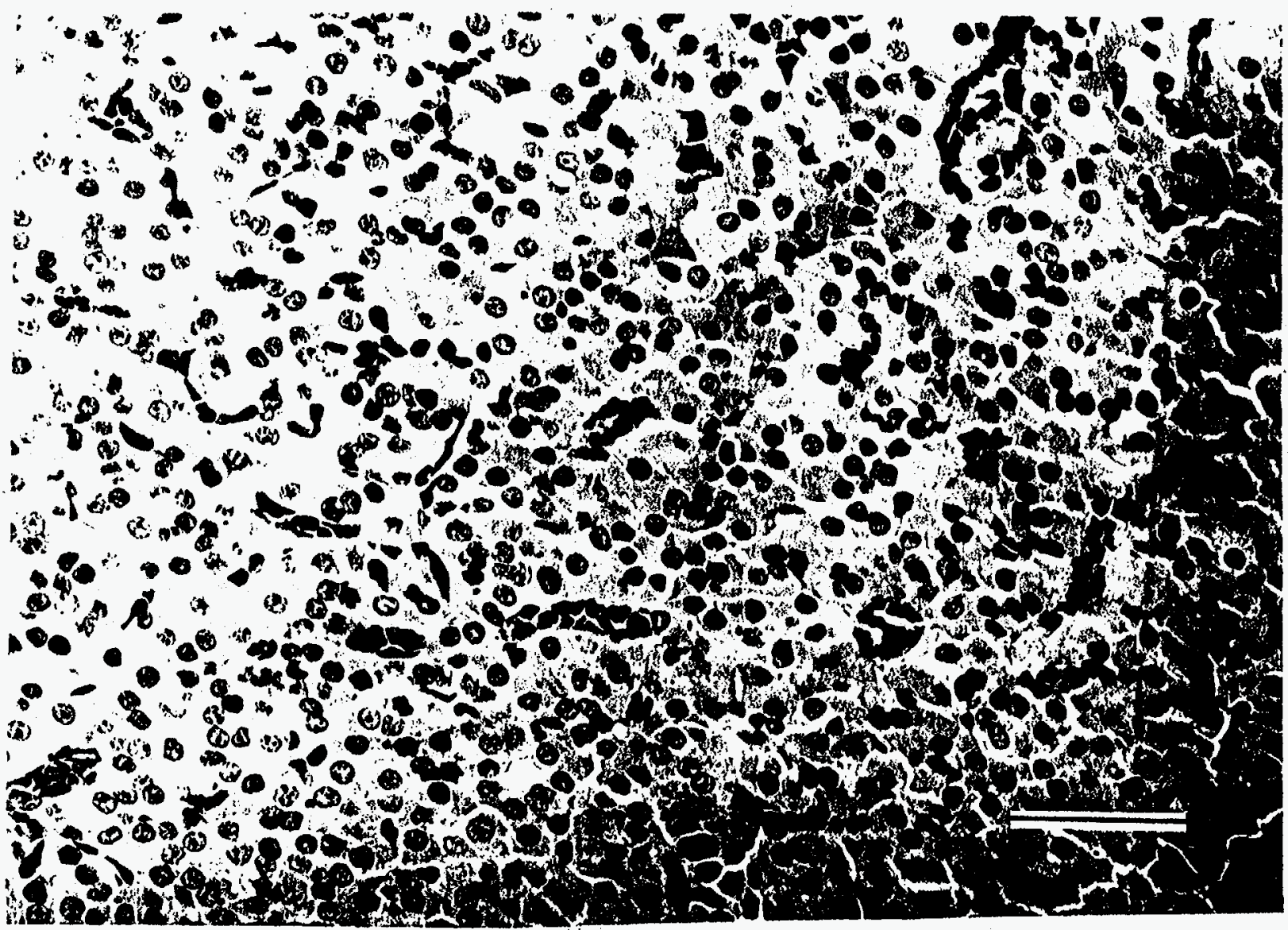


Thyroid

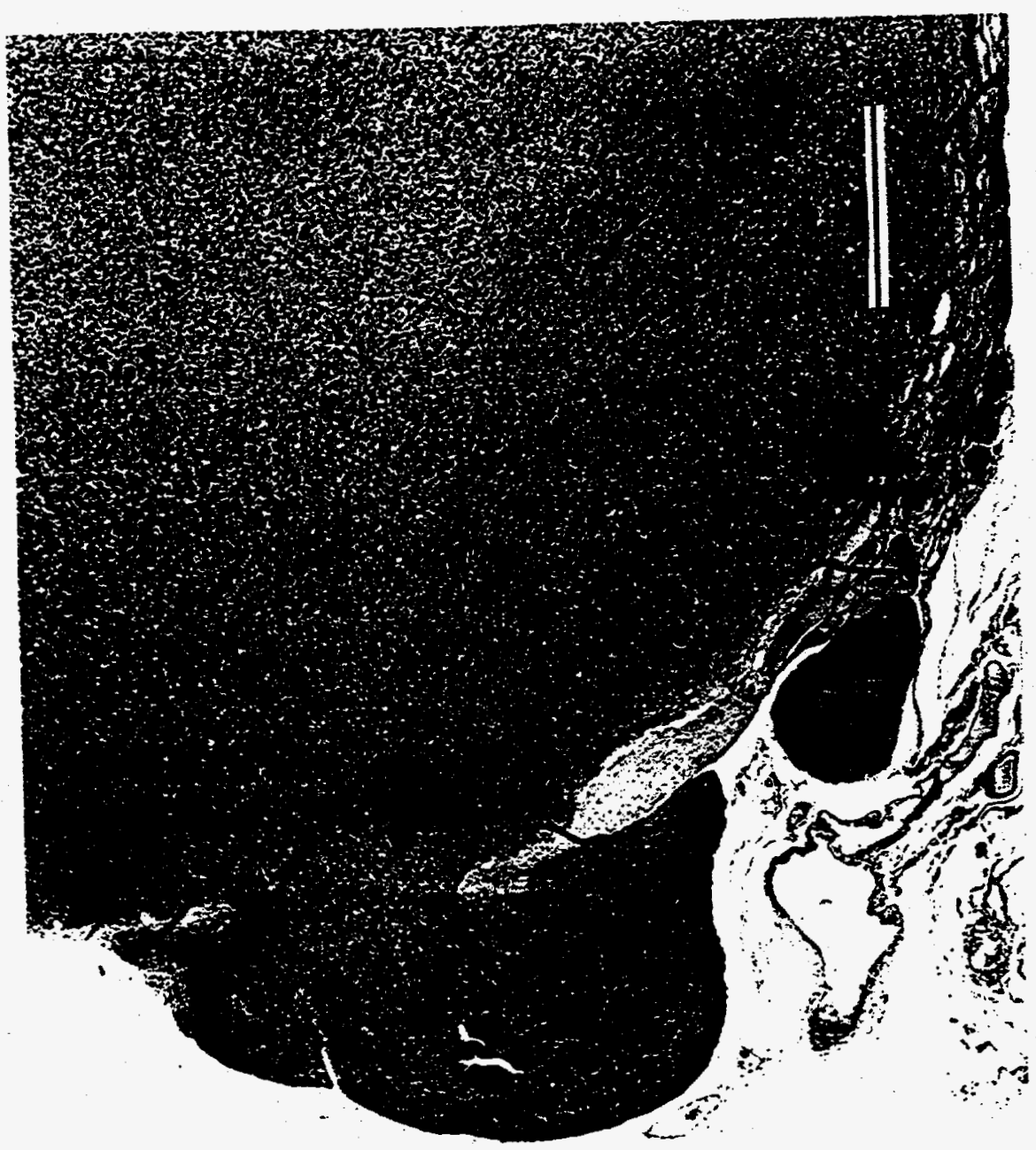

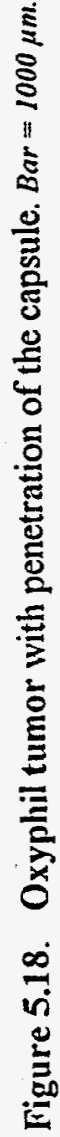


Thyroid

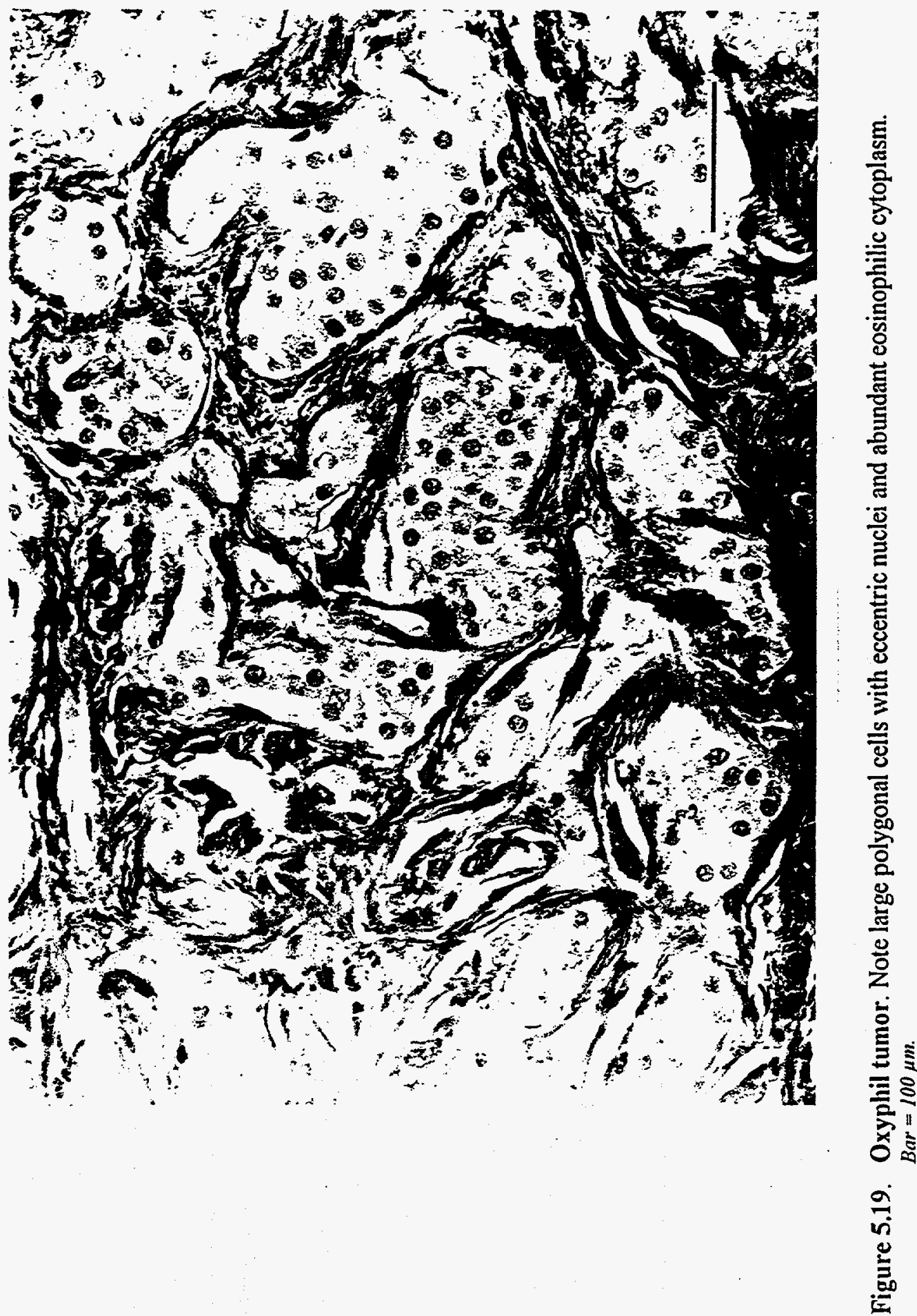




\title{
APPENDIX
}

\section{SNOMED Coded Database}

\author{
Charles R. Watson \\ National Radiobiology Archives \\ Pacific Northwest National Laboratory
}

The authors of this Atlas, in cooperation with the National Radiobiology Archives, are recording their observations in coded form. The glossary of codes is called SNODOG (Watson 1993a,b); it is a beagle-specific extension of the SNOVET (1984) glossary. SNOVET was derived from the Systematized Nomenclature of Medicine (SNOMED 1979, 1982, 1993), which was derived from the Systematized Nomenclature of Pathology (SNOP 1965).

SNOMED is a systematized multiaxial nomenclature of medically useful terms, hierarch-ically organized, where possible, and derived from the basic categories of SNOP. The axes are: Topography, Morphology, Etiology, Function, Disease, Procedure, and Occupation; they are identified by the prefix letter T, M, E, F, D, P, or $O$ in the code. Within an axis, five-character numeric codes are assigned in a hierarchical manner.

SNOVET is a microglossary, or subset, of SNOMED terms applicable to veterinary medicine. The authors of SNOVET added many animal-specific terms, inserting them into the SNOMED numeric hierarchy in the gaps left purposefully for such use. For example, humans do not have tails nor get distemper.

These glossaries were adopted by the DOE laboratories performing long-term studies of beagle dogs at various stages of glossary completion and at various points in time. Each laboratory independently developed computational procedures for handling the codes, and each expanded the glossary to fit research-specific circumstances. For example, neither SNOMED nor SNOVET provided codes for more than two mammary glands, and since mammary tumors are rather common in the beagle, and since beagles have eight mammary glands, each laboratory developed a unique coding system to identify them.

In the late $1980 \mathrm{~s}$, the SNODOG glossary was developed to integrate these diverse approaches. The Laboratory for Energy-Related Health Research, University of California at Davis, had made extensive use of SNOMED codes and had added and used a sixth digit in the numeric portion of the codes. Therefore, SNODOG is based on a six-digit code. A master data tape was made, containing every term in the SNOMED and SNOVET glossaries (with a zero appended) and the beagle-specific terms with a $1,2,3, \ldots$ appended. This tape was distributed to the laboratories and is the standard for information exchange between them and the National Radiobiology Archives. The SNODOG glossary is maintained and coordinated by the Archives; it is revised and distributed periodically as new terms are added (Watson 1993a). Table A.1 illustrates the various codes. 
Table A.1 Comparison of Selected Terms in Medical Glossaries

\begin{tabular}{|c|c|c|c|c|}
\hline Term & SNOP & SNOMED & SNOVET & SNODOG \\
\hline Rib (NOS) & T1133 & $\mathrm{T} 10350$ & $\mathrm{~T} 10350$ & $\mathrm{~T} 103500$ \\
\hline First rib & $\mathrm{n} / \mathrm{a}$ & T10351 & $\mathrm{T} 10351$ & $\mathrm{~T} 103510$ \\
\hline Thirteenth rib & $\mathrm{n} / \mathrm{a}$ & $\mathrm{n} / \mathrm{a}$ & $\mathrm{n} / \mathrm{a}$ & $\mathrm{T} 103631$ \\
\hline Adrenal gland (NOS) & T9300 & T93000 & T93000 & T930000 \\
\hline Adrenal capsule & T9303 & T93030 & T93030 & $\mathrm{T} 930300$ \\
\hline Extracapsular area of adrenal & $\mathrm{n} / \mathrm{a}$ & $\mathrm{n} / \mathrm{a}$ & $\mathrm{n} / \mathrm{a}$ & T930311 \\
\hline $\mathrm{X}$-zone of adrenal gland & $\mathrm{n} / \mathrm{a}$ & $\mathrm{n} / \mathrm{a}$ & T93165 & T931650 \\
\hline Inflammation & $\mathrm{M} 4000$ & M40000 & $\mathrm{M} 40000$ & M400000 \\
\hline Inflammation, granulomatous & M4400 & M44000 & M44000 & M440000 \\
\hline Lick granuloma & $\mathrm{n} / \mathrm{a}$ & $\mathrm{n} / \mathrm{a}$ & $\mathrm{n} / \mathrm{a}$ & M440001 \\
\hline
\end{tabular}

Pathologists' observations are usually coded as a combination of Topography and Morphology, with occasional use of the other axes. Often, there will be multiple database entries for a given topography code, as it may take several medical terms to describe a particular lesion. Each of the lifespan beagle project laboratories adopted distinct methods of coding observations as described by Watson (1993a). Table A.2 illustrates typical SNODOG coded patholgy observations.

Table A.2 SNODOG-Coded Pathologists' Observations

\begin{tabular}{|c|c|c|}
\hline Description & $\begin{array}{c}\text { Topography } \\
\text { Code }\end{array}$ & $\begin{array}{c}\text { Morphology } \\
\text { Code }\end{array}$ \\
\hline Lung Tumor & $\mathrm{T} 280000$ & $\mathrm{M} 800130$ \\
\hline Inflamed prostate gland w/ cystic hyperplasia & $\mathrm{T} 771000$ & $\begin{array}{l}\text { M401020 } \\
\text { M730610 }\end{array}$ \\
\hline Emphysema and fibrosis of the left cardiac lung lobe & $\mathrm{T} 287801$ & $\begin{array}{l}\text { M343010 } \\
\text { M480510 }\end{array}$ \\
\hline Nephrosis & $\mathrm{T} 710000$ & M531000 \\
\hline Adenocarcinoma, left kidney & T7 10200 & M814030 \\
\hline Radiation pneumonitis & $\mathrm{T} 280000$ & $\begin{array}{l}\text { M116200 } \\
\text { M497701 }\end{array}$ \\
\hline
\end{tabular}

Table A. 3 contains the major pathological terms defined in this Atlas, sorted alphabetically. Also shown are the corresponding SNODOG code and standardized nomenclature and the chapter in which the term is discussed. Table A.4 contains the same information as Table A.3, sorted alphabetically by the translation of the SNODOG term. Table A.5 is similar, sorted numerically by SNODOG code. These three tables provide multiple indexes to the atlas and serve as a guide to researchers approaching this information through the databases maintained by the National Radiobiology Archives. 
Table A.3 SNODOG Codes and Standardized Nomenclature with Terminology used in the Atlas sorted by Terminology used in Atlas

SNODOG Standardized Nomenclature

M81403B Bronchial adencarcinoma, malignant

M814000 Adenoma, benign

M81400B Bronchial adenoma, benign

M724901 Hyperplasia, adenomatous

M856030 Adenosquamous carcinoma, malignant

M802030 Carcinoma, undifferentiated type, malignant

M801230 Large cell carcinoma, malignant

M800230 Tumor, malignant, small cell type

M844000 Cystadenoma, benign

M825030 Bronchiolo-alveolar adenocarcinoma, malignant

M851030 Medullary carcinoma (C-cell carcinoma), malignan

M824000 Carcinoid tumor, benign

M918130 Chondroblastic osteosarcoma, malignant

M922030 Chondrosarcoma, malignant

M807030

M984031

M984030

M918230

M881000

M881030

M833000

M833030

M72000F

M925030

M918630

M912030

M817030

M580005

M816000

M824030

M816030

M889030

M885030

M430000

Combined osteosarcoma, malignant

Squamous cell carcinoma, malignant (epidermoid carcinoma)

Erythremic myelosis

Erythroleukemia, malignant

Fibroblastic osteosarcoma, malignant

Fibroma, benign

Fibrosarcoma, malignant

Follicular adenoma, benign

Follicular adenocarcinoma, malignant

Hyperplasia, follicular

Giant cell tumor of bone, malignant

Giant cell osteosarcoma, malignant

Hemangiosarcoma (Angiosarcoma), malignant

Hepatocellular carcinoma, malignant

Atrophy, follicular

Bile duct adenoma, benign

Carcinoid tumor, malignant

Cholangiocarcinoma (Bile duct carcinoma), malignant

Leiomyosarcoma, malignant

Liposarcoma, malignant

Inflammation, chronic
Chapter Terminology used in Atlas

4 Adenocarcinoma, Bronchial gland

4 Adenoma

4 Adenoma, Bronchial gland

4 Adenomatous hyperplasia

4 Adenosquamous carcinoma

4,5 Anaplastic carcinoma

4 Anaplastic carcinoma, Large cell

4 Anaplastic carcinoma, Small cell

3 Benign intrahepatic cystadenoma

4 Bronchiolavleolar carcinoma

5 C-cell carcinoma

4 Carcinoid tumor, benign

1 Chondroblastic Osteosarcoma

1,4 Chondrosarcoma

1 Combined-Type Osteosarcoma

4,5 Epidermoid carcinoma

2 Erythremic myelosis (FA B-EM)

2 Erythroleukemia (FAB-M6)

1 Fibroblastic Osteosarcoma

4 Fibroma

1,3,4 Fibrosarcoma

5 Follicular adenoma

5 Follicular carcinoma

5 Follicular hyperplasia

1 Giant Cell Tumor of Bone

1 Giant Cell Type Osteosarcoma

1,3,4 Hemangiosarcoma

3 Hepatic cell carcinoma

5 Ideopathic follicular atrophy

3 Intrahepatic bile duct adenoma

3,4 Intrahepatic carcinoid

3 Intrahepatic cholangiocarcinoma

4 Leiomyosarcoma

1 Liposarcoma

5 Lymphocytic thyroiditis 
Table A.3 SNODOG Codes and Standardized Nomenclature with Terminology used in the Atlas sorted by Terminology used in Atlas

SNODOG Standardized Nomenclature

M961030 Lymphosarcoma, malignant

M974030 Mast cell sarcoma, malignant

M991032 Megakaryocytic leukemia with maturation, malignant

M991031 . Megakaryocytic leukemia without maturation, malignant

M905030 Mesothelioma, malignant

M905000 Mesothelioma, benign

M894000 Mixed tumor (Pleomorphic adenoma), benign

M894030 Mixed tumor, malignant (AFIP 80850)

M918732 Osteoblastic osteosarcoma, moderately productive, malignant

M989032 Monocytic leukemia with maturation, malignant

M989031 Monocytic leukemia without maturation, malignant

M490005 Myelofibrosis

M986132 Myeloid leukemia with maturation, malignant

Myelomonocytic leukemia, malignant

M884000 Myxoma, benign

M884030 Myxosarcoma, malignant

M720300 Hyperplasia, nodular

M918731 Osteoblastic osteosarcoma, non-productive, malignant

M918730 Osteoblastic osteosarcoma, malignant

M918030 Osteosarcoma, malignant

M829030 Oxyphilic adenocarcinoma, malignant, primary site

M826030 Papillary adenocarcinoma, malignant

M826000 Papillary adenoma, benign

M805030 Papillary carcinoma, malignant

M973030 Plasma cell myeloma, malignant

M918032 Ostcosarcoma, poorly differentiated, malignant,

M918733 Ostcoblastic osteosarcoma, productive, malignant

M560002 Ostcodystrophy, radiation induced

M497701 Inflammation, pneumonitis, radiation induced

M964030 Reticulosarcoma (Histocytic Lymphosarcoma), malignant

M823030 Solid carcinoma, malignant

M823033 Solid-follicular carcinoma, malignant

M724903 Hyperplasia, squamous

M918330 Telangiectatic osteosarcoma, malignant

Chapter Terminology used in Atlas

1 Lymphosarcoma

3 Mast cell sarcoma

2 Megakaryocytic leukemia with maturation (FAB-M7b)

2 Megakaryocytic leukemia without maturation (FA B-M7a)

3,4 Mesothelioma

4 Mesothelioma, benign

4 Mixed tumor, benign

4 Mixed tumor, malignant

1 Moderately Productive Osteoblastic Osteosarcoma

2 Monocytic leukemoa with maturation (FAB-M5b)

2 Monocytic leukemoa without maturation (FAB-M5a)

2 Myelofibrosis

2 Myeloid leukemia with maturation (FAB-M2)

2 Myeloid leukemia without maturation (FAB-M1)

2 Myelomonocytic lẹkemia (FAB-M4)

3 Myxoma

3 Myxosarcoma

3 Nodular hyperplasia

1 Nonproductive Osteoblastic Osteosarcoma

1 Osteoblastic Osteosarcoma

1,4 Osteosarcoma

5 Oxyphil tumor

4 Papillary adenocarcinoma

4 Papillary adenoma

5 Papillary carcinoma

1 Plasma Cell Myeloma (Multiple Myeloma)

1 Poorly Differentiated Osteosarcoma

I Productive Osteoblastic Osteosarcoms

I Ridiation Ostcodystrophy

4 Radiation pneumonitis

1 Reticulum Cell Sarcoma (Histocytic Lymphoma)

4,5 Solid carcinoma

5 Solid-follicular carcinoma

4 Squamous metaplasia

1 Telangiectatic Osteosaroma 
Table A.4 SNODOG Codes and Standardized Nomenclature with Terminology used in the Atlas sorted by Standardized Nomenclature

SNODOG Standardized Nomenclature

\begin{tabular}{|c|c|}
\hline M814000 & Adenoma, benign \\
\hline M856030 & Adenosquamous carcinoma, malignant \\
\hline M580005 & Atrophy, follicular \\
\hline M816000 & Bile duct adenoma, benign \\
\hline M81403B & Bronchial adencarcinoma, malignant \\
\hline M81400B & Bronchial adenoma, benign \\
\hline M825030 & Bronchiolo-alveolar adenocarcinoma, malignant \\
\hline M824000 & Carcinoid tumor, benign \\
\hline M824030 & Carcinoid tumor, malignant \\
\hline M802030 & Carcinoma, undifferentiated type, malignant \\
\hline M816030 & Cholangiocarcinoma (Bile duct carcinoma), malignant \\
\hline M918130 & Chondroblastic osteosarcoma, malignant \\
\hline M922030 & Chondrosarcoma, malignant \\
\hline M918530 & Combined osteosarcoma, malignant \\
\hline M844000 & Cystadenoma, benign \\
\hline M984031 & Erythremic myelosis \\
\hline M984030 & Erythroleukemia, malignant \\
\hline M918230 & Fibroblastic osteosarcoma, malignant \\
\hline M881000 & Fibroma, benign \\
\hline M881030 & Fibrosarcoma, malignant \\
\hline M833030 & Follicular adenocarcinoma, malignant \\
\hline M833000 & Follicular adenoma, benign \\
\hline M918630 & Giant cell osteosarcoma, malignant \\
\hline M925030 & Giant cell tumor of bone, malignant \\
\hline M912030 & Hemangiosarcoma (Angiosarcoma), malignant \\
\hline M817030 & Hepatocellular carcinoma, malignant \\
\hline M724901 & Hyperplasia, adenomatous \\
\hline $\mathrm{M} 72000 \mathrm{~F}$ & Hyperplasia, follicular \\
\hline M720300 & Hyperplasia, nodular \\
\hline M724903 & Hyperplasia, squamous \\
\hline M430000 & Inflammation, chronic \\
\hline M497701 & Infammation, pneumonitis, radiation induced \\
\hline M801230 & Large cell carcinoma, malignant \\
\hline M889030 & Leiomyosarcoma, malignant \\
\hline M885030 & Liposarcoma, malignant \\
\hline M961030 & Lymphosarcoma, malignant \\
\hline
\end{tabular}

Chapter Terminology used in Atlas

4 Adenoma

4 Adenosquamous carcinoma

5. Ideopathic follicular atrophy

3 Intrahepatic bile duct adenoma

4 Adenocarcinoma, Bronchial gland

4 Adenoma, Bronchial gland

4 Bronchiolavlcolar carcinoma

4 Carcinoid tumor, benign

3,4 Intrahepatic carcinoid

4,5 Anaplastic carcinoma

3 Intrahepatic cholangiocarcinoma

1 Chondroblastic Osteosarcoma

1,4 Chondrosarcoma

1 Combined-Type Osteosarcoma

3 Benign intrahepatic cystadenoma

2 Erythremic myelosis (FAB-EM)

2 Erythroleukemia (FAB-M6)

1 Fibrablastic Osteosarcoma

4 Fibroma

1,3,4 Fibrosarcoma

5 Follicular carcinoma

5 Follicular adenoma

1 Giant Cell Type Osteosarcoma

1 Giant Cell Tumor of Bone

1,3,4 Hemangiosarcoma

3 Hepatic cell carcinoma

4 Adenomatous hyperplasia

5 Follicular hyperplasia

3 Nodular hyperplasia

4 Squamous metaplasia

5 Lymphocytic thyroiditis

4 Radiation pneumonitis

4 Anaplastic carcinoma, Large cell

4 Leiomyosarcoma

1 Liposarcoma

1 Lymphosarcoma 
Table A.4 SNODOG Codes and Standardized Nomenclature with Terminology used in the Atlas sorted by Standardized Nomenclature

SNODOG Standardized Nomenclature

Chapter Terminology used in Atlas

\begin{tabular}{|c|c|c|}
\hline M961030 & Lymphosarcoma, malignant & 1 Lymphosarcoma \\
\hline M974030 & Mast cell sarcoma, malignant & 3 Mast cell sarcoma \\
\hline M851030 & Medullary carcinoma (C-cell carcinoma), malignant & 5 C-cell carcinoma \\
\hline M991032 & Megakaryocytic leukemia with maturation, malignant & 2 Megakaryocytic leukemia with maturation (FAB-M7b) \\
\hline M991031 & Megakaryocytic leukemia without maturation, malignant & 2. Megakaryocytic leukemia without maturation (FAB-M7a \\
\hline M905000 & Mesothelioma, benign & 4 Mesothelioma, benign \\
\hline M905030 & Mesothelioma, malignant & 3,4 Mesothelioma \\
\hline M894000 & Mixed tumor (Pleomorphic adenoma), benign & 4 Mixed tumor, benign \\
\hline M894030 & Mixed tumor, malignant (AFIP 80850) & 4 Mixed tumor, malignant \\
\hline M989032 & Monocytic leukemia with maturation, malignant & 2 Monocytic leukemoa with maturation (FAB-M5b) \\
\hline M989031 & Monocytic leukemia without maturation, malignant & 2 Monocytic leukemoa without maturation (FAB-M5a) \\
\hline M490005 & Myelofibrosis & 2 Myelofibrosis \\
\hline M986132 & Myeloid leukemia with maturation, malignant & 2 Myeloid leukemia with maturation (FAB-M2) \\
\hline M986131 & Myeloid leukemia without maturation, malignant & 2 Myeloid leukemia without maturation (FAB-M1) \\
\hline M986031 & Myelomonocytic leukemia, malignant & 2 Myelomonocytic leukemia (FAB-M4) \\
\hline M884000 & Myxoma, benign & 3. Myxoma \\
\hline M884030 & Myxosarcoma, malignant & 3 Myxosarcoma \\
\hline M918730 & Ostcoblastic osteosarcoma, malignant & I Ostcoblastic Osteosarcoma \\
\hline M918732 & Osteoblastic osteosarcoma, moderately productive, malignan & 1 Moderately Productive Osteoblastic Osteosarcoma \\
\hline M918731 & Osteoblastic osteosarcoma, non-productive, malignant & 1 Nonproductive Osteoblastic Osteosarcoma \\
\hline M918733 & Osteoblastic osteosarcoma, productive, malignant & 1 Productive Osteoblastic Osteosarcoma \\
\hline M560002 & Osteodystrophy, radiation induced & 1 Radiation Osteodystrophy \\
\hline M918030 & Osteosarcoma, malignant & 1,4 Osteosarcoma \\
\hline M918032 & Osteosarcoma, poorly differentiated, malignant, & I Poorly Differentiated Osteosarcoma \\
\hline M829030 & Oxyphilic adenocarcinoma, malignant, primary site & 5 Oxyphil tumor \\
\hline M826030 & Papillary adenocarcinoma, malignant & 4 Papillary adenocarcinoma \\
\hline M826000 & Papillary adenoma, benign & 4 Papillary adenoma \\
\hline M805030 & Papillary carcinoma, malignant & 5 Papillary carcinoma \\
\hline M973030 & Plasma cell myeloma, malignant & I Plasma Cell Mycloma (Multiplc Mycloma) \\
\hline M964030 & Reticulosarcoma (Histocytic Lymphosarcoma), malignant & I Reticulum Cell Sarcoma (Histocytic Lymphoma) \\
\hline M823030 & Solid carcinoma, malignant & 4,5 Solid carcinoma \\
\hline M823033 & Solid-follicular carcinoma, malignant & 5 Solid-follicular carcinoma \\
\hline M807030 & Squamous cell carcinoma, malignant (epidermoid carcinoma & 4,5 Epidermoid carcinoma \\
\hline M918330 & Telangiectatic osteosarcoma, malignant & 1 Telangiectatic Osteosaroma \\
\hline M800230 & Tumor, malignant, small cell type & 4 Anaplastic carcinoma, Small cell \\
\hline
\end{tabular}


Table A.5 SNODOG Codes and Standardized Nomenclature with Terminology used in the Atlas sorted by SNODOG Code

M81403B Bronchial adencarcinoma, malignant

M814000 Adenoma, benign

M81400B Bronchial adenoma, benign

M724901 Hyperplasia, adenomatous

M856030 Adenosquamous carcinoma, malignant

M802030 Carcinoma, undifferentiated type, malignant

M801230 Large cell carcinoma, malignant

M800230 Tumor, malignant, small cell type

M844000 Cystadenoma, benign

M825030 Bronchiolo-alveolar adenocarcinoma, malignant

M851030 Medullary carcinoma (C-cell carcinoma), malignant

M824000 Carcinoid tumor, benign

M918130 Chondroblastic osteosarcoma, malignant

M922030 Chondrosarcoma, malignant

M918530 Combined osteosarcoma, malignant

M807030 Squamous cell carcinoma, malignant (epidermoid carcinoma)

M984031 Erythremic myelosis

M984030 Erythroleukemia, malignant

M918230 Fibroblastic osteosarcoma, malignant

M881000 Fibroma, benign

M881030 Fibrosarcoma, malignant

M833000 Follicular adenoma, benign

M833030 Follicular adenocarcinoma, malignant

M72000F Hyperplasia, follicular

M925030 Giant cell tumor of bone, malignant

M918630 Giant cell osteosarcoma, malignant

M912030 Hemangiosarcoma (Angiosarcoma), malignant

M817030 Hepatocellular carcinoma, malignant

M580005 Atrophy, follicular

M816000 Bile duct adenoma, benign

M824030 Carcinoid tumor, malignant

M816030 Cholangiocarcinoma (Bile duct carcinoma), malignant

M889030 Leiomyosarcoma, malignant

M885030 Liposarcoma, malignant

M430000 Inflammation, chronic
4 Adenocarcinoma, Bronchial gland

4 Adenoma

4 Adenoma, Bronchial gland

4 Adenomatous hyperplasia

4 Adenosquamous carcinoma

4,5 Anaplastic carcinoma

4 Anaplastic carcinoma, Large cell

4 Anaplastic carcinoma, Small cell

3 Benign intrahepatic cystadenoma

4 Bronchiolavleolar carcinoma

5 C-cell carcinoma

4 Carcinoid tumor, benign

1 Chondroblastic Osteosarcoma

1,4 Chondrosarcoma

1 Combined-Type Osteosarcoma

4,5 Epidermoid carcinoma

2 Erythremic myelosis (FAB-EM)

2 Erythroleukemia (FAB-M6)

1 Fibroblastic Osteosarcoma

4 Fibroma

1,3,4 Fibrosarcoma

5 Follicular adenoma

5 Follicular carcinoma

5 Follicular hyperplasia

1 Giant Cell Tumor of Bone

1 Giant Cell Type Osteosarcoma

1,3,4 Hemangiosarcoma

3 Hepatic cell carcinoma

5 Ideopathic follicular atrophy

3 Intrahepatic bile duct adenoma

3,4 Intrahepatic carcinoid

3 Intrahepatic cholangiocarcinoma

4 Leiomyosarcoma

1 Liposarcoma

5 Lymphocytic thyroiditis 
Table A.5 SNODOG Codes and Standardized Nomenclature with Terminology used in the Atlas sorted by SNODOG Code

M961030 Lymphosarcoma, malignant

M974030 Mast cell sarcoma, malignant

M991032 Megakaryocytic leukemia with maturation, malignant

M991031 Megakaryocytic leukemia without maturation, malignant

M905030 Mesothelioma, malignant

M905000 Mesothelioma, benign

M894000 Mixed tumor (Pleomorphic adenoma), benign

M894030 Mixed tumor, malignant (AFIP 80850)

M918732 Osteoblastic osteosarcoma, moderately productive, malignant

M989032 Monocytic leukemia with maturation, malignant

M989031 Monocytic leukemia without maturation, malignant

M490005 Myelofibrosis

M986132 Myeloid leukemia with maturation, malignant

M986131 Myeloid leukemia without maturation, malignant

M986031 Myelomonocytic leukemia, malignant

M884000 Myxoma, benign

M884030 Myxosarcoma, malignant

M720300 Hyperplasia, nodular

M918731 Osteoblastic osteosarcoma, non-productive, malignant

M918730 Osteoblastic osteosarcoma, malignant

M918030 Osteosarcoma, malignant

M829030 Oxyphilic adenocarcinoma, malignant, primary site

M826030 Papillary adenocarcinoma, malignant

M826000 Papillary adenoma, benign

M805030 Papillary carcinoma, malignant

M973030 Plasma cell myeloma, malignant

M918032 Osteosarcoma, poorly differentiated, malignant,

M918733 Osteoblastic osteosarcoma, productive, malignant

M560002 Osteodystrophy, radiation induced

M497701 Inflammation, pneumonitis, radiation induced

M964030 Reticulosarcoma (Histocytic Lymphosarcoma), malignant

M823030 Solid carcinoma, malignant

M823033 Solid-follicular carcinoma, malignant

M724903 Hyperplasia, squamous

M918330 Telangiectatic osteosarcoma, malignant
1 Lymphosarcoma

3 Mast cell sarcoma

2 Megakaryocytic leukemia with maturation (FAB-M7b)

2 Megakaryocytic leukemia without maturation (FAB-M7a)

3,4 Mesothelioma

4 Mesothelioma, benign

4 Mixed tumor, benign

4 Mixed tumor, malignant

1 Moderately Productive Osteoblastic Osteosarcoma

2 Monocytic leukemoa with maturation (FAB-M5b)

2 Monocytic leukemoa without maturation (FAB-M5a)

2 Myelofibrosis

2 Myeloid leukemia with maturation (FAB-M2)

2 Myeloid leukemia vithout maturation (FAB-M1)

2 Myelomonocytic leukemia (FAB-M4)

3 Myxoma

3 Myxosarcoma

3 Nodular hyperplasia

1 Nonproductive Osteoblastic Osteosarcoma

1 Osteoblastic Osteosarcoma

1,4 Osteosarcoma

5 Oxyphil tumor

4 Papillary adenocarcinoma

4 Papillary adenoma

5 Papillary carcinoma

1 Plasma Cell Myeloma (Multiple Myeloma)

I . Poorly Differentiated Osteosarcoma

1 Productive Osteoblastic Osteosarcoma

I Radiation Ostcodystrophy

4 Radiation pneumonitis

1 Reticulum Cell Sarcoma (Histocytic Lymphoma)

4,5 Solid carcinoma

5 Solid-follicular carcinoma

4 Squamous metaplasia

1 Telangiectatic Osteosaroma 


\section{REFERENCES}

SNOMED. 1979. Systematized Nomenclature of Medicine, Coding Manual, GE Gantner, RA Cotte, and RS Beckett, Eds. College of American Pathologists, Skokie, IL.

SNOMED. Systematized Nomenclature of Medicine, 2nd edition, updated through 1982. 1982. Volume 1, Numeric Index; Volume 2, Alphabetic Index. College of American Pathologists, Skokie, IL.

SNOMED International. Systematized Nomenclature of Human and Veterinary Medicine, $3^{\text {rd }}$ edition. 1993. Volume 1 and 2, Numeric Index; Volume 3 and 4, Alphabetic Index. RA Côté, DJ Rothwell, JL Palotay, RS Beckett, and L Brochu, Eds. College of American Pathologists, Northfield, IL.

SNOP. Systematized Nomenclature of Pathology. 1965. College of American Pathologists, Skokie, IL.

SNOVET. Systematized Nomenclature of Veterinary Medicine. 1984. Microglossary for Veterinary Medicine, James L Palotay and David J Rothwell, Eds. American Veterinary Medical Association, Schaumburg, IL.

Watson, C.R. 1993a. SNODOG Glossary, Part I: Introduction.PNL-8650 Pt. 1, Pacific Northwest Laboratory, Richland, WA.

Watson, C.R. 1993b. SNODOG Glossary, Part II: Usage of Terms, PNL-7934, Pacific Northwest Laboratory, Richland, WA, 459 pp. 
Acronyms 


\section{ACRONYMS}

ACHE acetylcholinesterase

ANL Argonne National Laboratory

CSU Colorado State University

DOE U.S. Department of Energy

FAB French-American-British (system for the classification of acute myeloid leukemia in man)

H\&E Harris hematoxylin and eosin

HH Harris hematoxylin

ITRI Inhalation Toxicology Research Institute

MPO myeloperoxidase

NRA National Radiobiology Archives

NSE nonspecific esterose (alpha naphthyl acetate)

OHER Office of Health and Environmental Research

PAS periodic acid-Schiff

PNNL Pacific Northwest National Laboratory

SNODOG Beagle specific variant of SNOMED created by NRA

SNOMED Systematized Nomenclature of Medicine

SNOP Systematized Nomenclature of Pathology

SNOVET Systematized Nomenclature of Veterinary Medicine

USTUR U.S. Transuranium and Uranium Registries

W-G Wright-Giemsa (stain)

WHO World Health Organization

WSU-TC Washington State University-Tri Cities 
Index 


\section{SUBJECT INDEX}

Adenocarcinoma

Brochioloalveolar

$103,109-110$

Bronchial gland

105,127

Papillary

103,111

Solid carcinoma

103,112

Adenoma

Bile duct

$84-86$

Bronchial gland 105,127

Cystic $133,142-143$

Follicular 132,139

Intrahepatic 84-86

Lung $105,123-124$

Papillary

Solid, thyroid 124

Thyroid

$133,140-141$

Adenomatous hyperplasia

$132-133,139-143$

Adenosquamous carcinoma

Anaplastic carcinoma

Lung

103,114

Thyroid 134

Brochioloalveolar carcinoma

Bronchial gland

$103,109-110$

Adenocarcinoma

105,127

Adenoma

Bile duct adenoma

C-Cell Carcinoma

86-88

Thyroid

Carcinoid

Intrahepatic

95

Lung 104,122

Lung, benign 105

Carcinoma

Adenosquamous

103,113

Anaplastic

103,134

Brochioloalveolar

105,110

C-Cell 134

Epidermoid

$101,107-108$

Follicular, thyroid 133,144

Hepatic cell

$87-88$

Large-cell

103,114

Lung, solid 103,112

Papillary 134

Small-cell 103

Solid

$103,112,133,145-147$

Solid follicular

$133,148-152$

Squamous

Thyroid

$133,144-152$ 
Index

Carcinoma

Undifferentiated

Cholangiocarcinoma

Intrahepatic

89-92

Chondroblastic osteosarcoma

$4,17-18$

Chondrosarcoma

Bone

Lung

Combined carcinoma

103,112

Combined-type osteosarcoma

$5,23-24$

Cystadenoma, intrahepatic

Cystic adenoma

Thyroid

$133,142-143$

Ectopic Tumor

Thyroid

Epidermoid carcinoma

Lung

Erythremic myelosis

$36,61-62$

Erythroleukemia

$36,56-60$

Fibroblastic osteosarcoma

4,19

Fibroma

Lung

105,125

Fibrosarcoma

Bone

Liver

Lung

104,116

Follicular adenoma

Thyroid

$132,139-141$

Follicular atrophy

Idiopathic

Thyroid

Follicular carcinoma

Thyroid

Follicular hyperplasia

Thyroid

Giant cell type osteosarcoma

Hemangiosarcoma

Bone

Intrahepatic

Liver

Lung

Hepatic cell carcinoma

Histocytoma

Fibrous (malignant)

Lung

Hypergranular promyelocytic leukemia

Hyperplasia

Follicular

Liver

Lung, Adenomatous 
Hyperplasia

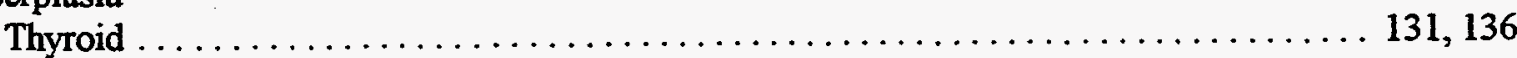

Idiopathic follicular atrophy

Thyroid

131,137

89-92

Intrahepatic cholangiocarcinoma

103,114

Large cell carcinoma

104,118

Lung .6

Liposarcoma

Bone 132,138

Lymphocytic thyroiditis

Lymphoid tumor

Bone

Mast cell sarcoma

Liver

Megakaryocytic leukemia

With maturation

$36,65-69,72-73$

Without maturation

$36,63-64$

Mesothelioma

Liver

Lung

105,128

Metaplasia

Squamous

105

Mixed tumor

Lung, benign .

105,126

Lung, malignant

104,121

Monocytic leukemia

With maturation

$35,54-55$

Without maturation

$35,48-53$

Multilobular tumor

Bone

Myelofibrosis

$36,70-71$

Myeloid leukemia

With maturation

$35,42-44$

Without maturation

$35,39-41$

Myelomatous tumor

Bone

Myelomonocytic leukemia

Myxoma

Intrahepatic

Myxosarcoma

Intrahepatic

steoblastic osteosarcoma

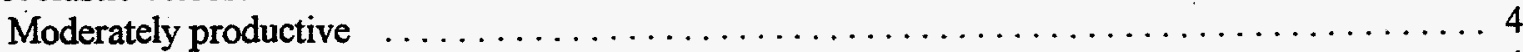

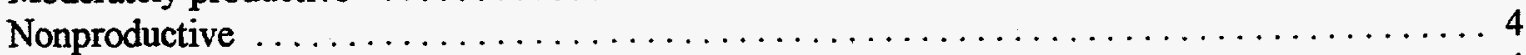

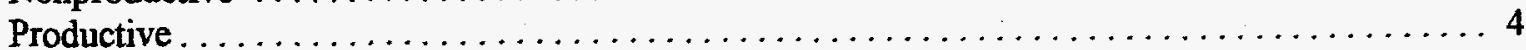

Osteodystrophy

Radiation 
Index

Carcinoma

Undifferentiated

Cholangiocarcinoma

Intrahepatic

$89-92$

Chondroblastic osteosarcoma

$4,17-18$

Chondrosarcoma

Bone

$5,25-27$

Lung

104,117

Combined carcinoma

103,112

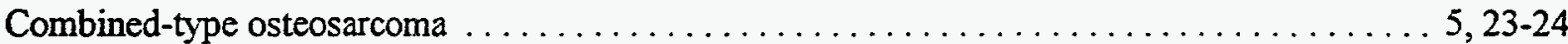

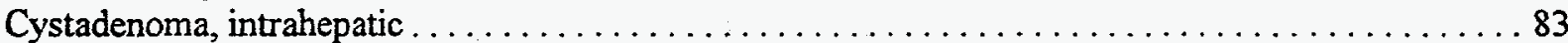

Cystic adenoma

Thyroid .

$133,142-143$

Ectopic Tumor

Thyroid

Epidermoid carcinoma

Lung

$101,107-108$

Erythremic myelosis

$36,62-63$

Erythroleukemia

$36,57-61$

Fibroblastic osteosarcoma

4,19

Fibroma

Lung

105,125

Fibrosarcoma

Bone

$5,28-30$

Liver

Lung

104,116

Follicular adenoma

Thyroid

$132,139-141$

Follicular atrophy

Idiopathic

131,137

Thyroid

131,137

Follicular carcinoma

Thyroid

133,144

Follicular hyperplasia

Thyroid

131,136

Giant cell type osteosarcoma

.. 5,22

Hemangiosarcoma

Bone

5,31

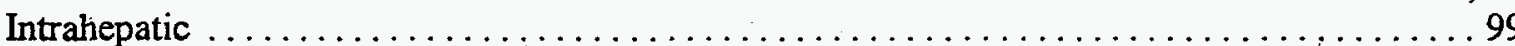

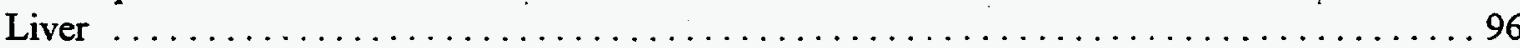

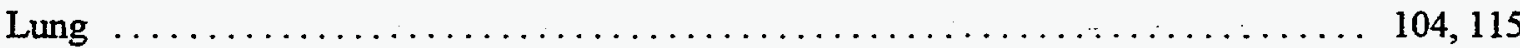

Hepatic cell carcinoma . . . . . . . . . . . . . . . .

Histocytoma

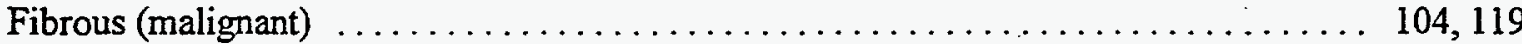

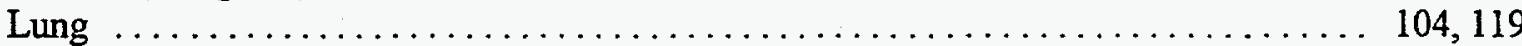

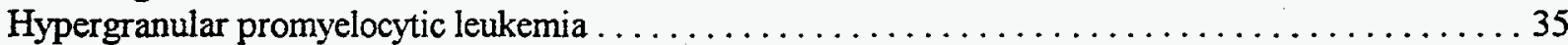

Hyperplasia

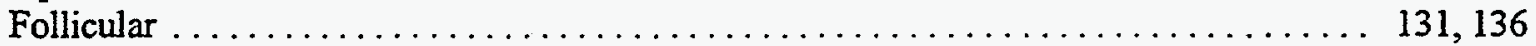

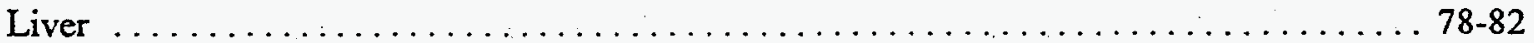

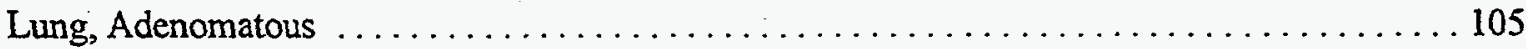


Hyperplasia

Thyroid

Idiopathic follicular atrophy

Thyroid

Intrahepatic cholangiocarcinoma

Large cell carcinoma

Leiomyosarcoma

Lung 104,118

Liposarcoma

Bone .. 6

Lymphocytic thyroiditis

132,138

Lymphoid tumor

Bone

Mast cell sarcoma

Liver

Megakaryocytic leukemia

With maturation

$36,66-70,73-74$

Without maturation

Mesothelioma

Liver

Lung 105,128

Metaplasia

Squamous

Mixed tumor

Lung, benign

Lung, malignant

Monocytic leukemia

With maturation

Without maturation

Multilobular tumor

Bone

Myelofibrosis

$36,71-72$

Myeloid leukemia

With maturation

Without maturation

Myelomatous tumor

Bone

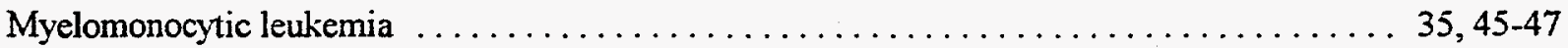

Myxoma

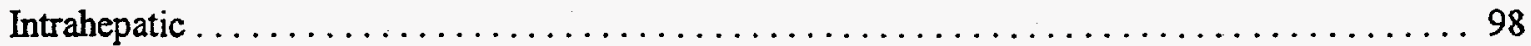

Myxosarcoma

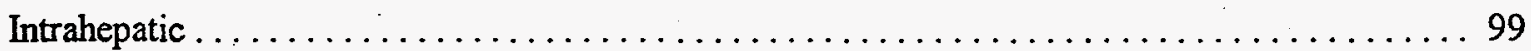

Osteoblastic osteosarcoma

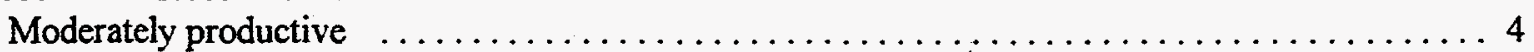

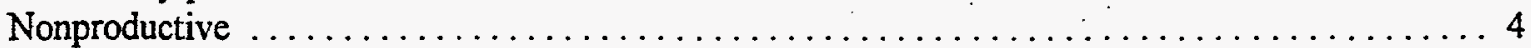

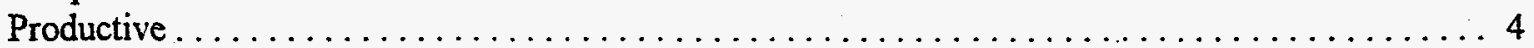

Osteodystrophy

Radiation 
Index

Osteosarcoma

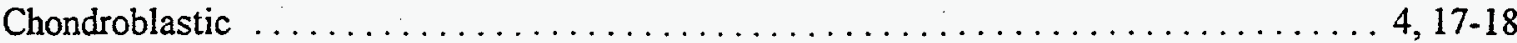

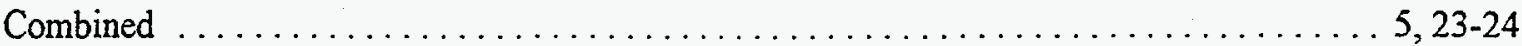

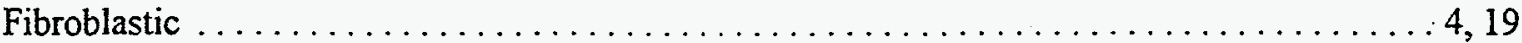

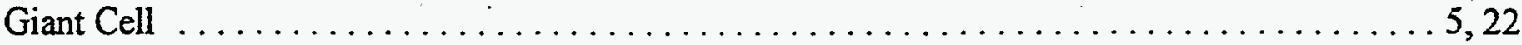

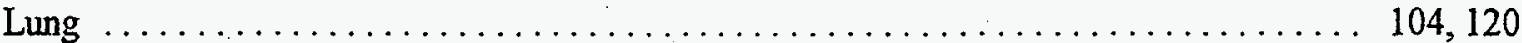

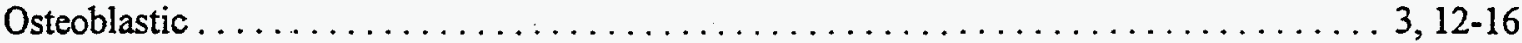

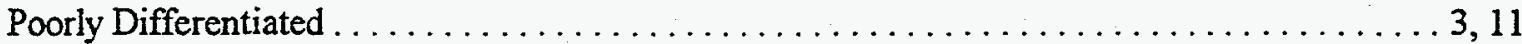

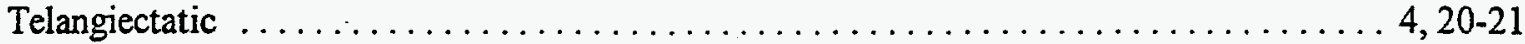

Oxyphil tumor

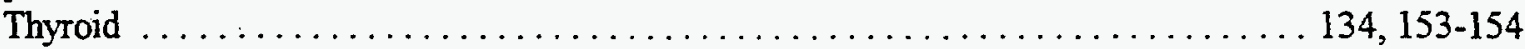

Papillary

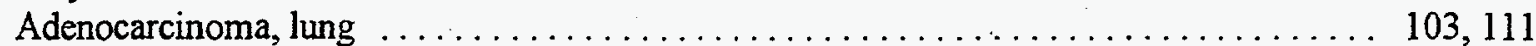

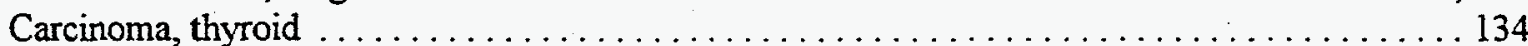

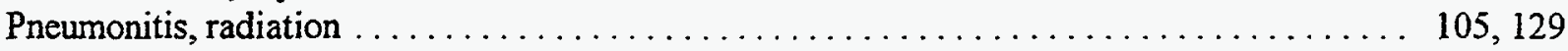

Radiation

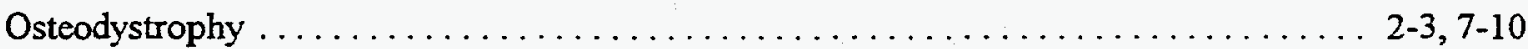

Pneumonitis ........................................ 105, 129

Sarcoma

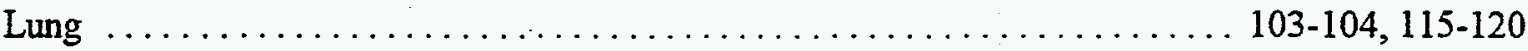

Solid adenoma

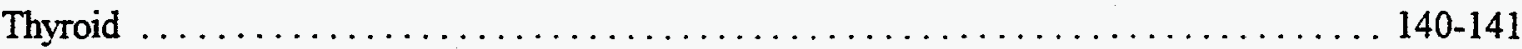

Solid carcinoma

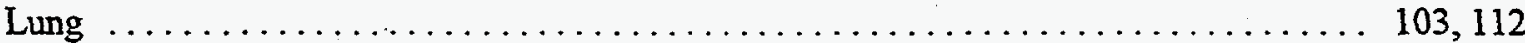

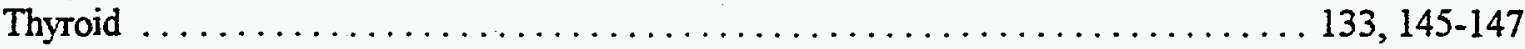

Solid follicular carcinoma

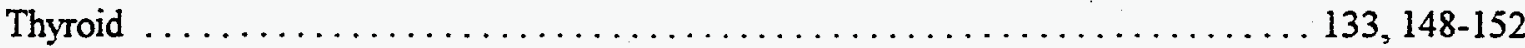

Squamous cell carcinoma

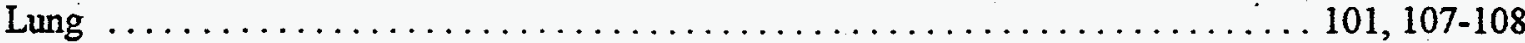

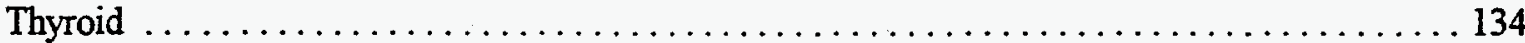

Squamous metaplasia

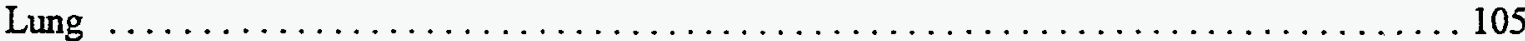

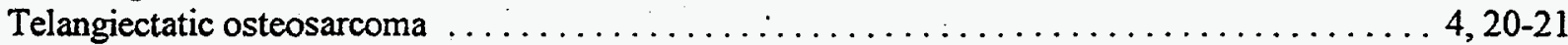

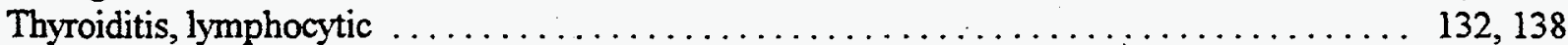

Udifferentiated carcinoma

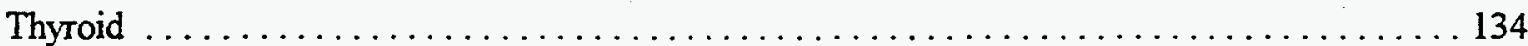


Index

\section{AUTHOR INDEX}

Author, address as of August, 1996

Chapter and pages

Benjamin, Stephen A., D.V.M., Ph.D. CET/CRHL

Foothills Campus

Colorado State University

Fort Collins, CO 80523

Dagle, Gerald E., D.V.M., Ph.D.

U.S. Transuranium and Uranium Registries

Preface: $\mathrm{i}$

Washington State University-Tri Cities

100 Sprout Road

Richland, WA 99352

Fritz, Thomas E., D.V.M.

Argonne National Laboratory

Radiation-Induced Myeloid Leukemia: $33-74$

9700 South Cass Ave.

Argonne, IL 60439

Gillett, Nancy A., D.V.M., Ph.D.

Tumors of Bone: 1-31

Sierra Biomedical, Inc.

587 Dunn Circle

Sparks, NV 89431

Haley, Patrick J., D.V.M, Ph.D.

Nycomed Inc

466 Devon Park Drive

PO Box 6630

Wayne, PA 19087-8630

Hahn, Fletcher F., D.V.M., Ph.D.

Inhalation Toxicology Research Institute

Lovelace Biomedical and Research Institute PO Box 5890

Albuquerque, NM 87185-5890

Muggenburg, Bruce A., D.V.M., Ph.D.

Tumors of Bone: 1-31

Inhalation Toxicology Research Institute

Lovelace Biomedical and Research Institute

PO Box 5890

Albuquerque, NM 87185-5890

Pool, Roy D., D.V.M, Ph.D.,

Tumors of the Lung: 101-129

Tumors of the Thyroid: 131-154

Tumors of Bone: $1-31$

Tumors of the Thyroid: 131-154

Tumors of the Lung: 101-129

Consolidatd Veterinary Diagnostics, Inc.

Tumors of Bone: 1-31

2825 KOVR drive

West Sacramento, CA 95605 
Index

Seed, Thomas M., Ph.D.

Armed Forces Radiobiology Research Institute

Radiation-Induced Myeloid Leukemia: 33-74 8901 Wisconsin Avenue

Bethesda, MD 20889

Taylor, Glenn N., D.V.M, Ph.D.

Tumors of the Liver: 75-100

Department of Radiobiology

Building 586

University of Utah

Salt Lake City, UT 84112

Tolle, David V., M.S.

Argonne National Laboratory

9700 South Cass Ave.

Argonne, IL 60439

Watson, Charles R, Ph.D.

Pacific Northwest National Laboratory

Preface: ii-iii

PO Box 999

Radiation-Induced Myeloid Leukemia: 33-74

Richland, WA 99352 